\title{
Autonome Feldroboter in der Landwirtschaft: Akzeptanz und Nutzungsbereitschaft
}

\author{
Dissertation \\ zur Erlangung des Doktorgrades \\ der Fakultät für Agrarwissenschaften \\ der Georg-August-Universität Göttingen
}

vorgelegt von

Friedrich Rübcke von Veltheim

geboren in Kaltenkirchen

Göttingen, im September 2021 
D 7

1. Referentin/Referent:

Prof. Dr. Ludwig Theuvsen

2. Korreferentin/Korreferent: Prof. Dr. Achim Spiller

Tag der mündlichen Prüfung: $\quad$ 08. November 2021 


\section{Inhalt}

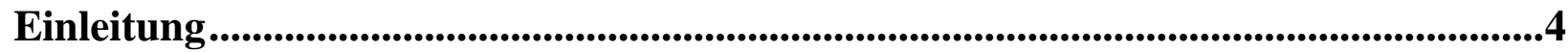

\section{Teil I:}

\section{Elektromobilität und Einsatz autonomer Feldroboter in der}

Landwirtschaft aus Sicht deutscher Landwirte

I.1 Elektromobilität in der Landwirtschaft - Eine qualitative Analyse zur

Nutzerakzeptanz.....

I.2 Akzeptanz autonomer Feldroboter im Ackerbaueinsatz - Status quo und Forschungsbedarf 55

I.3 Geman Farmers' Willingness to Adopt Autonomous Field Robots: An Empirical Survey

I.4 Geman Farmers' Intention to Use Autonomous Field Robots: A PLS-Analysis..108

\section{Teil II:}

Einsatz autonomer Feldroboter in der Landwirtschaft aus Sicht von Landmaschinenherstellern

II.1 Autonomous Field Robots in Agriculture: A Qualitative Analysis of User Acceptance According to Different Agricultural Machinery Companies

II.2 The AgTech Startup Perspective to Farmers ex-ante Acceptance of Autonomous Field Robots

\section{Teil III:}

Strukturwandel und dezentrale Energieversorgung in der deutschen

Landwirtschaft.

III.1 Die gesellschaftliche Wahrnehmung von bäuerlicher und industrieller Landwirtschaft 193

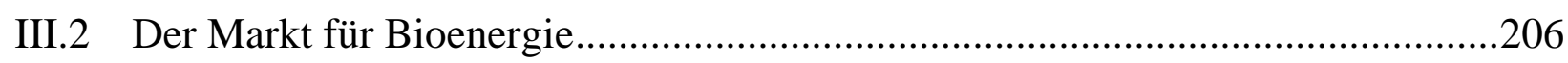

Schlussbetrachtung und Ausblick ..................................................................................238

Veröffentlichungs- und Vortragsverzeichnis ................................................................251

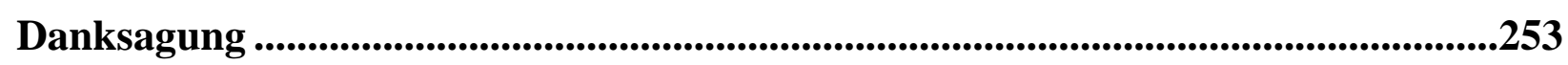

Eidesstattliche Erklärungen.....................................................................................255 


\section{Einleitung}

„,Nichts ist so beständig wie der Wandel“ (Heraklit von Ephesus, 535-475 v. Chr.)

Die Landwirtschaft gilt als klassisches Beispiel einer Automatisierung von Produktionsprozessen, die sich über die industriellen Revolutionen der vergangenen rund 200 Jahre erstreckt hat (BAUERNHANSL, 2014; LIU et al., 2021). Diese industriegeschichtlichen Umbrüche werden retrospektiv zumeist in vier Phasen unterteilt, die sich an maßgeblichen technologischen Durchbrüchen orientieren. Nach SCHWAB (2016) vollzog sich die erste industrielle Revolution in Europa von der Mitte des 18. bis zur Mitte des 19. Jahrhunderts; ihren Auslöser bildete die mechanische Nutzung von Wasser- und Dampfkraft. Auf sie folgte im beginnenden 20. Jahrhundert unter verstärkter Nutzung elektrischer Energie und mit Einführung von Fließband- und Massenproduktion die zweite industrielle Revolution. Die dritte industrielle Revolution geht zurück auf die Entwicklung von Halbleitern und Großrechnern ab den 1960er Jahren, denen Personalcomputer (seit den 1970er und 1980er Jahren) und das Internet (seit den 1990er Jahren) folgten. Die noch in den Anfängen steckende vierte industrielle Revolution wird durch die Verzahnung der industriellen Produktion mit modernster Informations- und Kommunikationstechnologie charakterisiert, die u.a. mithilfe autonom und hochpräzise arbeitender Maschinen Einzelfertigung in Qualität und Produktivität industrieller Massenfertigung ermöglicht (KROMBHOLZ, 2019).

Analog zu den industriellen lassen sich auch die landwirtschaftlichen bzw. -technischen Revolutionen - wenngleich etwas zeitversetzt - in vier Phasen unterteilen. Im Gegensatz zur Industrie waren die sozialen Transformationen des 19. Jahrhunderts ${ }^{1}$ jedoch nicht die Folge, sondern Ausgangspunkt der ersten landwirtschaftlichen Revolution (Landwirtschaft 1.0). Eine mögliche Unterteilung hat KROMBHOLZ (2019) vorgenommen:

- Landwirtschaft 1.0 (Anfang bis Mitte 20. Jahrhundert): bedeutende Zuchtfortschritte bei Nutzpflanzen und -tieren; Landtechnik zunehmend industriell gefertigt; Mechanisierung auf Basis Muskelkraft (v.a. des Pferdes); gemischte Betriebe

\footnotetext{
${ }^{1}$ siehe MARX (1867).
} 
(Nutzpflanze und -tier) mit Kreislaufwirtschaft dominieren; Anwendung von Dampfkraft zweitrangig, da eher in stationären als mobilen Bereichen einsetzbar

- Landwirtschaft 2.0 (ab Mitte 20. Jahrhundert): wissenschaftlich induzierte, deutliche Erweiterung landwirtschaftlicher Produktionsprozesse (u.a. durch Integration anderer Wissenschaftsbereiche); weitestgehend industriell gefertigte, komplexere Landtechnik; Motorisierung des mobilen Bereichs (Standardtraktor) auf Grundlage von Verbrennung führt zu grundlegender Veränderung der Pflanzenproduktionsprozesse; Elektrifizierung des stationären Bereichs; Wegfall von Zugtieren führt zu Ausweitung von Flächen für Nahrungsgüterproduktion und beginnende Entkopplung pflanzlicher von tierischer Produktion (Spezialisierung der Betriebe); Ertragssteigerung durch vermehrten Einsatz von Mineraldüngern und Pflanzenschutzmittel im Pflanzenbau

- Landwirtschaft 3.0 (ab Ende 20. Jahrhundert): analog zur Industrie 3.0 zunehmende Anwendung von Mikroelektronik und Informationstechnologie („Precision Agriculture“); erste Automatisierungen, wie Melkroboter oder Global Positioning-System (GPS)-Lenksysteme; Produktivitätssteigerungen auf Grundlage wachsender Mechanisierungsmittel und Produktionseinheiten, wie Ackerfläche oder Tierplätze (,Wachstumsgesetz der Landtechnik“)

- Landwirtschaft 4.0 (aktuell oder künftig): Umkehrung der Charakteristik des „Wachstumsgesetzes der Landtechnik“ mithilfe modernster Kommunikations- und Informationstechnologie (z.B. vollautomatische Robotereinheiten); Alternativen zum derzeitigen Nähr- und Wirkstoffeinsatz

Während in der vorindustriellen Zeit noch alle notwendigen Arbeitsschritte (wie Bodenbearbeitung, Aussaat, Düngung, Schädlingsbekämpfung, Ernte und Drusch) manuell durchgeführt wurden, bewirkte der zunehmende Einsatz von Maschinen wie Traktoren und Mähdreschern einen immer höheren Automatisierungsgrad landwirtschaftlicher Produktionsprozesse. Als Folge wurden mehr und mehr landwirtschaftliche Arbeitsplätze durch effizientere Maschinen ersetzt. Zusätzlich machten es die neuen Maschinen möglich, größere Flächeneinheiten kosteneffizient zu bewirtschaften. Dadurch setzte ein über die Zeit an Geschwindigkeit gewinnender, tiefgreifender Strukturwandel in der Agrar- und Ernährungswirtschaft ein (BÄURLE und TAMÁSY, 2012). Neben den innerbetrieblichen 
Strukturänderungen beschleunigten die technischen Neurungen der einzelnen landwirtschaftlichen Revolutionen einen Prozess, den COCHRANE (1958) als „landwirtschaftliche Tretmühle“ beschrieben hat. Danach zwingen wenige, innovative Landwirtschaftsbetriebe durch Einsatz kosteneffizienterer Produktionsmethoden die Mehrheit der Landwirte ${ }^{2}$ dazu, diese über die Zeit ebenfalls zu adaptieren, um nicht Gefahr zu laufen, unrentabel zu werden und schließlich aus der Produktion aussteigen zu müssen. Die „landwirtschaftliche Tretmühle“ wird dabei als fortwährender Prozess der Anpassung an technische Neuerungen gesehen und führt zwangsläufig zur Zerschlagung bestehender und zur Schaffung neuer und effizienterer Strukturen. In Folge dieser „schöpferischen Zerstörung“ (SCHUMPETER, 1942) scheiden zunehmend Betriebe aus der Produktion aus und die Verbleibenden werden größer und spezialisieren sich stärker. Die damit verbundenen Produktivitätssteigerungen und Preissenkungen von relativ preisunelastischen ${ }^{3}$ Grundnahrungsmitteln wurden angesichts von Nahrungsmittelknappheit und -bedarf (vor allem während und nach Kriegsjahren oder Naturkatastrophen) von Politik und Gesellschaft breit unterstützt. Seit der Bedarf an Grundnahrungsmitteln in Industrieländern wie Deutschland jedoch weitestgehend gedeckt werden konnte, rücken Themen wie Nachhaltigkeit oder Tierwohl bei bestehenden landwirtschaftlichen Produktionsmethoden vermehrt in den Vordergrund öffentlicher Debatten und lösen mitunter breite Kritik an der konventionellen Landwirtschaft aus (SPILlER et al., 2015; VoERSTE, 2008; WoOdHOUSE, 2010). Diese Kritik geht in Teilen so weit, dass negative Auswirkungen auf die Reputation und die damit verbundene „licence to operate“ der Landwirte in Deutschland befürchtet werden (ERMANN et al., 2017).

Die pflanzliche Erzeugung hat gemessen an ihrem Produktionswertanteil (2020: rd. $49 \%$ bzw. 27 Mrd. €) die größte wirtschaftliche Bedeutung innerhalb der Landwirtschaft in Deutschland (BLE, 2020; DBV, 2021). Die gesellschaftliche Kritik entzündet sich mit Blick auf diesen Sektor vor allem am Einsatz chemischer Pflanzenschutzmittel, der unter anderem für den fortschreitenden Biodiversitätsverlust in der Agrarlandschaft direkt oder indirekt verantwortlich gemacht wird (JAHN et al., 2014). Zusätzlich führte eine teilweise

\footnotetext{
${ }^{2}$ Zur besseren Lesbarkeit findet in der vorliegenden Dissertation das generische Maskulinum Anwendung. Dies soll jedoch keinesfalls eine Geschlechterdiskriminierung oder eine Verletzung des Gleichheitsgrundsatzes zum Ausdruck bringen.

${ }^{3}$ Als preisunelastisch gilt ein Gut, wenn die relative Änderung seines Angebots oder seiner Nachfrage im Verhältnis zur relativen Änderung seines Preises kleiner Eins ist (MUßHOFF und HIRSCHAUER, 2020).
} 
Überdüngung landwirtschaftlicher Nutzflächen in Deutschland zur Erzeugung politischen Handlungsbedarfs ${ }^{4}$, der sich 2020 in Form einer strengeren Düngeverordnung äußerte, die von vielen Landwirte als Mehrbelastung wahrgenommen wird (BUNDESREGIERUNG, 2020). Daneben sorgen immer größere und lautere Landmaschinen besonders in Siedlungsnähe für Unmut in der Bevölkerung (SCHAPER et al., 2013). Auch die landwirtschaftlichen Betriebe selbst kämpfen mit den Folgen eines aufgrund zunehmender Resistenzen immer kleiner werdenden Spektrums wirksamer Komponenten in Pflanzenschutzmitteln sowie mit einer mitunter irreversiblen Bodenverdichtung durch $\mathrm{zu}$ schwere Landmaschinen (GAUS et al., 2017). Hinzu kommen weitere Herausforderungen, wie ein zunehmender Fachkräftemangel, der unter anderem dem landwirtschaftlichen Strukturwandel, der demographischen Entwicklung und der sich verringernden Akzeptanz landwirtschaftlicher Produktionsmethoden in Deutschland geschuldet ist (GINDELE et al., 2016), sowie zunehmende Ertrags- und Preisschwankungen aufgrund des Klimawandels und der Liberalisierung der Agrarmärkte (VON HOBE et al., 2021).

Ein möglicher Beitrag zur Lösung der beschriebenen Herausforderungen könnte von einer der jüngsten Entwicklungen der Landwirtschaft 4.0 geleistet werden: autonome Feldroboter (AFR). Diese hochautomatisierten Roboter haben das Potenzial, die nächste Generation landwirtschaftlicher Feldtechnik darzustellen. Sie können allein oder in intelligent vernetzten Schwärmen mithilfe von Sensoren unerwünschte Beikräuter erkennen und mechanisch oder mittels Applikation von Kleinstmengen chemischer Pflanzenschutzmittel präziser selektieren als menschliche Arbeitskräfte (HEUSER et al., 2018). Damit würden ein ressourceneffizienterer und umweltfreundlicherer Einsatz von Pflanzenschutzmitteln sowie eine bedarfsgerechtere, einzelpflanzenspezifische Düngung bei gleichzeitiger wirtschaftlicher Vorteilhaftigkeit in bestimmten Pflanzenkulturen ermöglicht werden (LOWENBERG-DEBOER et al., 2020). Dieser Aspekt wird umso bedeutsamer, je lauter die gesellschaftliche Kritik am Einsatz von Dünge- und chemischen Pflanzenschutzmitteln in der Landwirtschaft und je enger sich das Spektrum verfügbarer Wirkstoffe im chemischen Pflanzenschutz wird. AFR wären in der Lage, verschiedenste Nutzpflanzenkulturen innerhalb eines

\footnotetext{
${ }^{4}$ Der Europäische Gerichtshof urteilte am 21.06.2018, dass Deutschland gegen die Nitratrichtline (91/676/EWG) verstoßen hat. Daraufhin forderte die Europäische Kommission Deutschland auf, seine Düngeverordnung entsprechend anzupassen (EUROPÄISCHE KOMMISSION, 2019).
} 
Schlages auf Grundlage zuvor durch computergestützte Analysen unterschiedlicher Kartierungen (z.B. Bodenqualität oder Ertragskartierung) erstellter Potenzialkarten anzupflanzen und zu bewirtschaften. Diese als „spot farming“ bekannte Anbauweise bietet neben einer positiven Wirkung auf die Biodiversität auch die Möglichkeit, Synergieeffekte bestimmter Pflanzenkombinationen zu erzielen (GAUS et al., 2017). Da sich die Roboter durch kleinere Ausmaße und ein geringeres Gewicht auszeichnen als herkömmliche Landmaschinen, würden sowohl straßenverkehrsrechtliche Hürden als auch die Problematik der Bodenverdichtung entfallen. Zudem könnte durch einen Verzicht auf einen Fahrer bei bestimmten landwirtschaftlichen Arbeiten dem Fachkräftemangel in der Landwirtschaft begegnet werden und eine Kostendegression in arbeitskraftintensiven Bereichen, wie z.B. dem Obst- und Gemüseanbau, zum Tragen kommen. Ein weiterer Vorteil besteht in den Antriebssystemen von AFR. Da diese zumeist elektrisch betrieben werden, könnten die Landwirte Kraftstoff sparen und den betriebseigenen, dezentral erzeugten Strom aus erneuerbaren Energien zum Laden der AFR nutzen. Zudem würde sich mit Elektromotoren das Problem der Lärm- und Schadstoffemissionen lösen lassen. Angesichts der besseren Skalierbarkeit von AFR könnten kleinbäuerliche Betriebe, die bisher oft als unwirtschaftlich galten, durch den Einsatz solcher Roboter profitabler werden, was dem fortschreitenden Strukturwandel in der Landwirtschaft entgegenwirken könnte (KING, 2017; LOWENBERG-DEBOER et al., 2020). So könnten alle drei Säulen der Nachhaltigkeit (ökologisch, ökonomisch und sozial) neu ausbalanciert und die zwischen ihnen bestehenden Interessenskonflikte zum Teil überwunden werden.

Da jeder Fortschritt zwangsläufig eine Änderung des Bestehenden zur Folge hat, wird auch die Einführung autonomer Technologien in die Landwirtschaft, ganz im Sinne der ,schöpferischen Zerstörung“, von einigen Bedenken begleitet. So würde im Fall von AFR ein Fahrer im klassischen Sinne zwar wegfallen und damit der Arbeitskräftebedarf vor dem Hintergrund des bestehenden Fachkräftemangels reduziert werden; doch bedingt die befürchtete Komplexität von AFR neue Fähigkeiten zur sachgerechten Bedienung und Reparatur, die auf den Betrieben möglicherweise nicht ausreichend vorhanden sind (REDHEAD et al., 2015). DEVITT (2018) verweist darauf, dass es Landwirten an Vertrauen in die korrekte Funktionsweise von AFR mangelt und die erhoffte Arbeitserleichterung durch einen Mehraufwand an Kontrolle aufgewogen werden könnte. Sie argumentiert weiter, dass mit 
voranschreitender Übernahme landwirtschaftlicher Tätigkeiten durch Roboter Landwirte sukzessive ersetzt und ihre landwirtschaftlichen Fähigkeiten und ihre soziale Stellung zunehmend verloren gehen könnten. Daneben wird der Einsatz elektrischer Antriebssysteme bei Landmaschinen im Allgemeinen und AFR-Konzepten im Speziellen nicht unkritisch gesehen; sie bedürften einer nicht unerheblichen Anpassung des rechtlichen Rahmens (PICKEL, 2018). Hauptkritikpunkte aus ökonomischer Perspektive sind ein erhöhter Kostenaufwand, die Kombination relativ geringer Reichweiten mit zu langen Ladezeiten vor dem Hintergrund umweltbedingter, enger Einsatzzeitfenster sowie die umweltbelastende Entsorgung der ausgedienten Akkumulatoren (KAIRIES, 2013; PETERS et al., 2013).

Aus einer globaleren Perspektive betrachtet, besteht grundsätzlich bei jeder neuen Technologie die Gefahr, dass sich diese zunächst vor allem Betriebe in wohlhabenderen Ländern leisten können und die ohnehin schon niedrigere Wettbewerbsfähigkeit von Landwirten in ärmeren Ländern weiter verringert wird ${ }^{5}$ (FLEMING et al., 2018). Neben den Landwirten betrifft diese neue Technologie auch die Landtechnikhersteller. So nimmt KING (2017) beispielsweise an, dass etablierte Landtechnikhersteller in AFR eine mögliche Bedrohung ihrer Geschäftsmodelle sehen und auf die Verbreitung von AFR zunächst zurückhaltend reagieren könnten. Der Wettbewerb zwischen den einzelnen Landtechnikunternehmen könnte durch die neuen Möglichkeiten, Daten zu sammeln und auszuwerten, zusätzlich verstärkt werden (PHAM und STACK, 2017). Zudem sind hiermit Probleme des Datenschutzes und des Dateneigentums verbunden (SCHLEICHER und GRANDORFER, 2018). Schließlich besteht die Gefahr der Monopolisierung in der Landtechnikbranche, da mit der zunehmenden Bedeutung der Datensammlung und -verarbeitung Netzwerkeffekte entstehen und Märkte durch eine „,winner takes all, loser gets nothing“-Situation (SHAPIRO und VARIAN, 1999) gekennzeichnet sein können.

Trotz der genannten Bedenken besteht weitestgehend Konsens, dass autonome Systeme Einzug in die moderne Landwirtschaft finden werden; unklar ist lediglich noch, wann und in welchem Umfang dies geschehen wird (KING, 2017; PICKEL 2019; SPARROW und HOWARD, 2020). In Anlehnung an ROGERS (1995) entscheiden Individuen immer dann über

\footnotetext{
${ }^{5}$ ROGERS (1995) beschreibt dieses Phänomen als „Innovativeness-needs Paradox“, wonach immer die Individuen in einem sozialen System, die den größten Bedarf an einer technischen Innovation haben, diese als letzte übernehmen.
} 
die Adaption oder Ablehnung einer Innovation, wenn die Unsicherheit über die zu erwartenden Konsequenzen ein erträgliches Maß angenommen hat. Die Konsequenzen hängen wiederum von verschiedenen Erfolgsfaktoren ab, wie der Wirtschaftlichkeit oder der sich aus politischen Entscheidungen ergebenden Rechtslage zum Einsatz technischer Innovationen. Da Landwirte in ihrem Entscheidungsverhalten - wie alle anderen Individuen auch - dem verhaltensökonomischen Prinzip der „,bounded rationality“ (SIMON, 1956) unterliegen und nicht vollumfänglich entsprechend der Theorie des nutzenmaximierenden ,homo oeconomicus“ agieren (DABBERT und BRAUN, 2021; MUßHOFF et al., 2009; ZIMMERMANN, 2003), hängt die Nutzungsbereitschaft entscheidend von der Technologieakzeptanz ab. Aufgrund der Tatsache, dass es sich bei einer Vielzahl von AFR um Prototypen oder Konzeptstudien handelt und erst wenige Produkte kommerzialisiert wurden, sind die Akzeptanz, der Prozess der Entstehung von Akzeptanz und der Einsatz dieser Technologie in der Landwirtschaft bislang kaum erforscht. Zahlreiche Studien konnten indes belegen, dass für eine erfolgreiche Entwicklung und Diffusion innovativer Technologien in der Landwirtschaft vor allem die Intention zur Nutzung entscheidend für den sich anschließenden Akzeptanzprozess ist (u.a. AMBONG und PAUlinO, 2020; AMIN und Li, 2014; FAR und RAZAEI-MOGHADDAM, 2015; REDHEAD et al., 2015; SCHUKAT et al., 2019).

Vor den genannten Hintergründen und aufgrund der bestehenden Forschungslücke beschäftigt sich die vorliegende Arbeit mit der Bereitschaft von Landwirten in Deutschland, AFR künftig zu nutzen. Ziel der Arbeit ist es, neben den Landwirten selbst auch die Sichtweise von Landmaschinenproduzenten, als Zulieferer und damit Stakeholder ${ }^{6}$ der Landwirtschaft, auf mögliche Akzeptanzfaktoren autonomer Feldtechnik unter Landwirten zu untersuchen, um auf diese Weise ein umfassenderes Bild des Akzeptanzprozesses zu erhalten. Dafür wird zunächst die Absicht zur Nutzung von Elektromobilität (E-Mobilität) durch Landwirte im Allgemein und von AFR im Speziellen betrachtet. Es folgt eine Analyse der betrachteten Nutzungsintention der Landwirte aus Sicht verschiedener Landmaschinenhersteller, unterteilt in etablierte Landtechnikunternehmen und AgTech Startups. Einerseits verfügen Landmaschinenhersteller über wertvolle Erfahrungen in Bezug auf das

\footnotetext{
${ }^{6}$ Als Stakeholder werden zentrale Anspruchsgruppen bezeichnet, die mit einer Organisation, Gesellschaft oder einem Produkt verbunden sind und daher Verantwortung gegenüber ihr/ihm und ein Interesse an ihrem/seinem Erfolg haben (CAMBRIDGE UNIVERSITY PRESS, 2021).
} 
Kaufverhalten ihrer Kunden; andererseits ist es strategisch wichtig bzw. sogar von existenzieller Bedeutung (AgTech Startups), den künftigen Erfolg von AFR richtig abzuschätzen. Auf diese Weise kann die Arbeit einen Beitrag zur empirischen Erforschung des Technikakzeptanzprozesses in der Landwirtschaft allgemein und in Bezug auf AFR im Speziellen leisten und gleichzeitig Impulse für eine Gestaltung künftiger AFR-Adaptions- und Diffusionsstrategien geben. Schließlich werden die gesellschaftliche Sichtweise auf die Wahrnehmung „bäuerlicher“ und ,industrieller“" Landwirtschaft allgemein sowie der Markt für Bioenergie in Form eines Exkurses betrachtet. Dafür wurde die Arbeit in drei Teile untergliedert, die in Tabelle 1 komprimiert dargestellt sind. Tabelle 1: Aufbau der Dissertation

\section{Teil I:}

Elektromobilität und Einsatz autonomer Feldroboter in der Landwirtschaft aus der Sicht deutscher Landwirte

\section{Einleitung}

I.1 Elektromobilität in der Landwirtschaft - Eine qualitative Analyse zur Nutzerakzeptanz

I.2 Akzeptanz autonomer Feldroboter im Ackerbaueinsatz Status quo und Forschungsbedarf

I.3 German Farmers' Willingness to Adopt Autonomous Field Robots: An Empirical Survey

I.4 German Farmers' Intention to Use Autonomous Field Robots: A PLS-Analysis
II.1 Autonomous Field Robots in Agriculture: A Qualitative Analysis of User Acceptance According to Different Agricultural Machinery Companies

II.2 The AgTech Startup Perspective to Farmers ex-ante Acceptance of Autonomous Field Robots

\section{Teil III:}

Strukturwandel und dezentrale

Energieversorgung in der deutschen Landwirtschaft

III.1 Die gesellschaftliche Wahrnehmung von bäuerlicher und industrieller Landwirtschaft

III.2 Der Markt für Bioenergie 2019

\section{Quelle: Eigene Darstellung}

\section{Schlussbetrachtung und Ausblick}

\section{Teil I: Elektromobilität und Einsatz autonomer Feldroboter in der Landwirtschaft aus Sicht deutscher Landwirte}

Im ersten Teil dieser Arbeit liegt der Fokus auf der Nutzungsintention landwirtschaftlicher Betriebsleiter in Deutschland in Bezug auf E-Mobilität allgemein und AFR im speziellen. Die verschiedenen Beiträge beruhen auf den Ergebnissen qualitativer Experteninterviews $(n=15)$ sowie einer standardisierten Online-Umfrage unter Landwirten $(n=500)$ in Deutschland, denen jeweils eine umfassende Literaturrecherche vorausging.

Landwirte sind als potenzielle Nutzer von AFR in Hinblick auf deren erfolgreichen Einsatz und die damit verbundene Diffusion die wichtigste Gruppe unter den betrachteten 
Stakeholdern. Der Einsatz von AFR in der Landwirtschaft und deren künftiger Marktanteil werden dabei vermutlich durch eine Vielzahl von Faktoren beeinflusst. In Anbetracht der eingangs angeführten Gründe für und gegen den Einsatz dieser Technologie ist hierbei die Nutzungsintention der Landwirte als Teil des Technologieakzeptanzprozesses besonders bedeutsam. So wird es kaum möglich sein, AFR erfolgreich in der Landwirtschaft zu etablieren, wenn ein Großteil der Landwirte nicht von ihrer Vorteilhaftigkeit überzeugt ist. DEVITT (2018) verweist darauf, dass ohne Evaluierung und anschließender Berücksichtigung von Akzeptanzfaktoren AFR möglicherweise nie das Stadium eines Nischenproduktes verlassen werden.

Beitrag I.2 „Elektromobilität in der Landwirtschaft - Eine qualitative Analyse zur Nutzerakzeptanz" untersucht durch Auswertung qualitativer Experteninterviews $(n=15)$ mögliche Akzeptanzfaktoren deutscher Landwirte, die den Einsatz von E-Mobilität in der Landwirtschaft beeinflussen. Im zweiten Beitrag „, Akzeptanz autonomer Feldroboter im Ackerbaueinsatz - Status quo und Forschungsbedarf" wird der Stand der Akzeptanzforschung zu AFR erhoben und aus Ermangelung einer ausreichenden Anzahl an Studien um Ergebnisse der Akzeptanzforschung zur sensorgestützten Präzisionslandwirtschaft ergänzt; schließlich werden Forschungsfragen für den weiteren Verlauf dieser Arbeit abgeleitet. Der dritte und der vierte Beitrag „German Farmers' Attitudes on Adopting Autonomous Field Robots: An Empirical Survey“ sowie „German Farmers' Intention to Use Autonomous Field Robots: A PLS-Analysis " schließlich befassen sich auf der Grundlage einer großzahligen Befragung mit der Nutzungsintention deutscher Landwirte $(n=500)$ in Bezug auf AFR. Dabei wird die untersuchte Stichprobe im dritten Beitrag mithilfe einer Clusteranalyse in unterschiedliche Zielgruppen für AFR segmentiert. In der letzten Studie dieses Kapitels wird anhand einer PLS-Analyse analysiert, inwieweit die latenten, intentionsbeeinflussenden Faktoren durch bestimmte Indikatoren beschrieben werden können, in welcher Beziehung sie zueinanderstehen sowie welche Stärke sie aufweisen. 


\section{Teil II: Einsatz autonomer Feldroboter in der Landwirtschaft aus Sicht von Land- maschinenherstellern}

Landmaschinenhersteller und AgTech Startups, die zweite in dieser Arbeit betrachtete Stakeholdergruppe, sind in der Regel sehr gut über den Stand der Forschung zu AFR informiert, da sie unmittelbar in deren Entwicklung und Produktion involviert sind. Für sie ist die Abschätzung des künftigen Erfolgs dieser neuen Technologie mindestens strategisch und in Teilen sogar existenziell bedeutsam. Über die Perspektive dieser Stakeholdergruppe hinsichtlich der Einflussfaktoren auf die Akzeptanz von AFR durch Landwirte ist jedoch bislang wenig bekannt. Daher befasst sich der zweite Teil dieser Arbeit mit der Sichtweise verschiedener etablierter Landmaschinenhersteller und AgTech Startups auf den AFR-Akzeptanzprozess bei Landwirten. Als Datengrundlage der beiden Beiträge fungieren qualitative Experteninterviews, deren Leitfäden Ergebnis einer umfangreichen Literaturanalyse waren.

Im ersten Beitrag dieses Kapitels „,Autonomous Field Robots in Agriculture: A Qualitative Analysis of User Acceptance According to Different Agricultural Machinery Companies “ wird auf Basis von Interviews mit neun führenden Landmaschinenherstellern in Deutschland deren Perspektive auf mögliche Einflussfaktoren auf die Akzeptanz von AFR unter Landwirten analysiert. Damit wird zusätzlich ein Beitrag zum Verständnis der oftmals vernachlässigten Multidimensionalität des Technikakzeptanzprozesses und der Komplexität der einzelnen Akzeptanzfaktoren in der Landwirtschaft geleistet (BUKCHIN und KERRET, 2018; PATHAK et al., 2019). Der zweite Beitrag des Kapitels „, The AgTech Startup Perspective to Farmers ex-ante Acceptance of Autonomous Field Robots " untersucht ebenfalls auf Grundlage von Experteninterviews mit verschiedenen europäischen AgTech Startups, die sich mit der Entwicklung von AFR beschäftigen, deren Sichtweise auf mögliche, den Akzeptanzprozess unter Landwirten beeinflussende Faktoren. Da für die meisten der betrachteten AgTech Startups, im Gegensatz zu den etablierten Landmaschinenherstellern, AFR keine mögliche Konkurrenz zu bestehenden Produktlinien und Geschäftsstrategien darstellen, erscheint eine vergleichende Betrachtung der jeweiligen Ergebnisse dieses Kapitels besonders interessant. 


\section{Teil III: Strukturwandel und dezentrale Energieversorgung in der deutschen Land- wirtschaft}

Der dritte und letzte Teil dieser Arbeit befasst sich im weiteren Sinne mit einigen Rahmenbedingungen zur erfolgreichen Etablierung von Technologien wie AFR in der Landwirtschaft. Dafür wird zunächst die Gesellschaft, die zunehmend an Einfluss auf landwirtschaftliche Produktionsmethoden gewinnt, als in hohem Maße relevante Stakeholdergruppe betrachtet. Daneben wird die Entwicklung des Marktes für Bioenergie untersucht, da die meisten der entwickelten AFR-Konzepte auf einen elektrischen Antrieb setzen, welcher nur dann als ökologisch nachhaltig gelten kann, wenn ihr Akkumulator die elektrische Energie zum Großteil aus erneuerbaren Quellen bezieht (AJANOvIC und HAAS, 2016; RIEDNER et al., 2019).

Im Zuge der agrarpolitischen und gesellschaftlichen Diskussion der vergangenen Jahre tauchen vermehrt die Begriffe einer „bäuerlichen“ und einer „,industriellen“ Landwirtschaft auf, wobei erstere oft als erhaltens- und erstrebenswert betrachtet wird, während letztere kritisch betrachtet wird (u.a. BULLER und MORRIS, 2004; CAMPBELL, 2009; GERKE, 2017; KAYSER et al., 2012; SPILLER et al., 2012). Nach einer kongruenten Verwendung geschweige denn einer einheitlichen Definition dieser beiden Begriffe sucht man jedoch vergebens. Daher beschäftigt sich der Beitrag III.1 „Die gesellschaftliche Wahrnehmung von bäuerlicher und industrieller Landwirtschaft" mit dem gesellschaftlichen Verständnis $(n=1.150)$ dieser beiden Begriffe in Deutschland. Grundlage dafür sind die Ergebnisse einer repräsentativen, standardisierten Online-Befragung von Konsumenten in Deutschland. Die Ergebnisse werden deskriptiv dar- und gegenübergestellt. Der zweite Beitrag dieses Kapitels „Der Markt für Bioenergie 2019“ analysiert die Entwicklung des Anteils der erneuerbaren Energien im Energiemix Deutschlands und wirft abschließend einen Blick auf Agrophotovoltaikanlagen als neuem Entwicklungsansatz innovativer, ressourceneffizienter Konzepte zur Doppelnutzung des knappen Faktors Boden. Solche Anlagen wären auch für AFR als dezentral verteilte Ladepunkte von besonderem Interesse. 


\section{Literatur}

AMIN, K. und J. LI (2014): Applying farmer technology acceptance model to understand farmer's behavior intention to use ICT based microfinance platform: A comparative analysis between Bangladesh and China. Wuhan International Conference on e-Business (WHICEB) Proceedings der AIS Electronic Library (AISeL), Wuhan, China.

AJANOVIC, A. und R. HAAS (2016): Dissemination of electric vehicles in urban areas: major factors for success. In: Energy 115: 1451-1458, doi: 10.1016/j.energy.2016.05.040.

BÄURLE, H. und C. TAMÁsY (2012): Regionale Konzentrationen der Nutztierhaltung in Deutschland. ISPA Mitteilungsheft 79, Vechta.

BAuernhansL, T. (2014): Die Vierte Industrielle Revolution - Der Weg in ein wertschaffendes Produktionsparadigma. In: BAuernhansl, T., Ten Hompel, M. und B. Vogel-Heuse (Hrsg.): Industrie 4.0 in Produktion, Automatisierung und Logistik. Springer Vieweg, Wiesbaden: 5-35, doi: 10.1007/978-3-65804682-8_1.

BLE (Bundesanstalt für Landwirtschaft und Ernährung) (2020): Landwirtschaftliche Produktionswert 2020: Nach erster Schätzung rund 56,3 Milliarden Euro. URL: https://www.ble.de/SharedDocs/Downloads/DE/Pressemitteilungen/2020/201216_Landwirtschaftlicher_Produktionswert.pdf;jsessionid=A943EEE80447E76AA38EC4E01841EB67.1_cid335?_blob_ =publicationFile\&v=2 (Abrufdatum: 15.04.2021).

BukChiN, S. und D. KERRET (2018): Food for Hope: The Role of Personal Resources in Farmers' Adoption of Green Technology. In: Sustainability 10 (5): 1615, doi: 10.3390/su10051615.

Buller, H. und C. MORRIS (2004): Growing goods: the market, the state, and sustainable food production. In: Environment and Planning A 36 (6): 1065-1108, doi: 10.1068/a35282.

BUNDESREGIERUNG (2020): Neue Düngeverordnung - Nährstoffeffizienz und saubere Gewässer. URL: https://www.bundesregierung.de/breg-de/aktuelles/duengeverordnung-1735878

(Abrufdatum: 15.04.2021).

CAMBRIDGE UNIVERSITY PRESS (2021): Cambridge Dictionary. URL: https://dictionary.cambridge.org/dictionary/english/stakeholder (Abrufdatum: 01.05.2021).

CAMPBELL, H. (2009): Breaking new ground in food regime theory: corporate environmentalism, ecological feedbacks and the 'food from somewhere' regime. In: Agriculture and Human Values 26 (4): 309-319, doi: 10.1007/ s10460-009-9215-8.

Cochrane, W. W. (1958): Farm Prices: Myth and Reality. University of Minnesota Press, Minneapolis.

DABBERT, S. und J. BRAUn (2021): Landwirtschaftliche Betriebslehre. Eugen Ulmer, Stuttgart.

DBV (Deutscher Bauernverband) (2021): Situationsbericht 2020/21. URL: https://www.bauernverband.de/situationsbericht/5-fakten-zur-wirtschaftlichen-lage-der-landwirtschaft-1/53-landwirtschaftliche-gesamtrechnung (Abrufdatum: 13.03.2021).

DeVITT, S. K. (2018): Cognitive Factors that Affect the Adoption of Autonomous Agriculture. In: Farm Policy Journal 15 (2): 49-60.

ERMANN, M., Christoph-SCHUlZ, I. und A. Spiller (2017): Under pressure - Wie nehmen Landwirtinnen und Landwirte in Deutschland den Druck externer Stakeholder wahr? In: Yearbook of the Austrian Society of Agricultural Economics 26: 85-94, doi: 10.24989/OEGA.JB.26.10.

EUROPÄISCHE KOMMISSION (2019): Nitrat im Grundwasser: Kommission mahnt Deutschland zur Umsetzung des EuGH-Urteils. URL: https://ec.europa.eu/germany/news/20190725-nitrat_de (Abrufdatum: 17.04.2021).

FAR, S. T. und K. REZAEI-MOgHADDAM (2017): Determinants of Iranian agricultural consultants' intentions toward precision agriculture: Integrating innovativeness to the technology acceptance model. In: Journal of the Saudi Society of Agricultural Sciences 16 (3): 280-286, doi: 10.1016/j.jssas.2015.09.003. 
Fleming, A., JAKKU, E., Lim-CAMACHO, L., TAYlOR, B. und P. THORBURN (2018): Is big data for big farming or for everyone? Perceptions in the Australian grains industry. In: Agronomy for Sustainable Development 38 (2018), doi: 10.1007/s13593-018-0501-y.

Gaus, C. C., T. F. Minßen, L.M. Urso, T. DE WitTE und J. Wegener (2017): Mit autonomen Landmaschinen zu neuen Pflanzenbausystemen. Schlussbericht. Johann Heinrich von Thünen-Institut, Bundesforschungsinstitut für Ländliche Räume, Wald und Fischerei; Technische Universität Braunschweig, Institut für mobile Maschinen und Nutzfahrzeuge; Julius-Kühn-Institut, Braunschweig.

GERKE, J. (2017): Bäuerliche Landwirtschaft. URL: http://ostdeutschebodenpolitik.de/2015/01/30/baeuerliche-landwirtschaft-zum-begriff-und-zu-ihrer-zukunft/ (Abrufdatum: 23.10.2017).

GINDELE, N., KAPS, S. und R. DOLUSCHITZ (2016): Betriebliche Möglichkeiten im Umgang mit dem Fachkräftemangel in der Landwirtschaft. In: Berichte über Landwirtschaft 94 (1), doi: 10.12767/buel.v94i1.89.

Heuser, S., M. DEMmel, J. Pfeiffer und M. GANDORFER (2018): Automatisierte mechanische Unkrautregulierung. In: Ruckelshausen, A., MeYer-Aurich, A., BOrchardt, K., Hofacker, C., LoY, J.-P., SChwerdtfeger, R., Sundermeier, H.-H., Floto, H. und B. Theuvsen (Hrsg.): Digitale Marktplätze und Plattformen. Lecture Notes in Informatics 278, Gesellschaft für Informatik, Bonn: 99-102.

JAhn, T., HÖTKER, H., OpPERMAnN, R., BLEIL, R. und L. VELE (2014): Protection of biodiversity of free living birds and mammals in respect of the effects of pesticides. Umweltbundesamt, Dessau-Roßlau.

KAIRIES, B. (2013): Marketing für Elektroautos. Akzeptanz als notwendige Bedingung für die Marktdurchdringung. Diplomica, Hamburg.

KAYSER, M., BÖHM, J. und A. SPILLER (2012): Zwischen Markt und Moral - Wie wird die deutsche Land- und Ernährungswissenschaft in der Gesellschaft wahrgenommen? In: Schriften der Gesellschaft für Wirtschafts- und Sozialwissenschaften des Landbaues e.V. 47: 329-341.

KING, A. (2017): Technology: The Future of Agriculture. In: Nature 544 (7651): 21-23, doi: 10.1038/544S21a.

KROMBHOLZ, K. (2019): Gedanken zur Vorgeschichte von Landwirtschaft 4.0. In: FrERICHS, L. (Hrsg.): Jahrbuch Agrartechnik 2018. Institut für mobile Maschinen und Nutzfahrzeuge, Braunschweig: 1-19.

LiU, Y., MA, X., Shu, L., Hancke, G. P. und A. M. AbU-Mahfouz (2021): From Industry 4.0 to Agriculture 4.0: Current Status, Enabling Technologies, and Research Challenges. In: IEEE Transactions on Industrial Informatics 17 (6): 4322-4334, doi: 10.1109/TII.2020.3003910.

Lowenberg-DeBoer, J., HuAng, I. Y., GrigOriAdis, V. und E. BlaCKMORE (2020): Economics of robots and automation in field crop production. In: Precision Agriculture 21: 278-299, doi: 10.1007/s11119019-09667-5.

MARX, K. (1867): Das Kapital - Kritik der politischen Ökonomie. Band I. Otto Meissner Verlag, Hamburg.

Mußhoff, O. und N. Hirschauer (2020): Modernes Agrarmanagement - Betriebswirtschaftliche Analyseund Planungsverfahren. 5. Auflage, Verlag Franz Vahlen, München.

Mubhoff, O., Hirschauer, N. und H. WAssmuss (2009): The Role of Bounded Rationality in Farm Financing Decisions - First Empirical Evidence -. Paper presented at the International Association of Agricultural Economists (IAAE) 2009 Conference, Beijing, China.

PATHAK, H. S., BROWN, P. und T. BEST (2019): A systematic literature review of the factors affecting the precision agriculture adoption process. In: Precision Agriculture 13 (9): 1-25, doi: 10.1007/s11119-01909653-x.

Peters, A., C. Doll, P. Plötz, A. Thielmann, A. Sauer, C. Zanker, M. Wietschel und W. Schade (2013): Konzepte der Elektromobilität. Ihre Bedeutung für Wirtschaft, Gesellschaft und Umwelt. Nomos Verlagsgesellschaft, Baden-Baden.

PHAM X. und M. STACK (2017): How data analytics is transforming agriculture. In: Business Horizons 61 (1): 125-133, doi: 10.1016/j.bushor.2017.09.011. 
PICKEL, P. (2018): Elektrifizierung in der Landwirtschaft - wo und wofür? In: Kuratorium für Technik und Bauwesen in der Landwirtschaft e. V. (Hrsg.): In Zukunft elektrisch - Energiesysteme im ländlichen Raum. Darmstadt: 56-74.

PICKEL, P. (2019): RoundTable: Agricultural robotics storms global market. Präsentation auf dem International Forum of Agricultural Robotics (FIRA) 2019, Toulouse.

Redhead, F., SNOW, S., Vyas, D., BAWden, O., Russell, R., Perez, T. und M. Brereton (2015): Bringing the Farmer Perspective to Agricultural Robots. In: Begole, B., KiM, J., InKPEN, K. und W. WoO (Hrsg.): Proceedings of the 33rd Annual ACM Conference Extended Abstracts on Human Factors in Computing Systems. Association for Computing Machinery, New York: 1067-1072, doi: 10.1145/2702613.2732894.

Riedner, L., Mair, C., ZimeK, M., BrudermanN, T. und T. STERn (2019): E-mobility in agriculture: differences in perception between experienced and non-experienced electric vehicle users. In: Clean Technology and Environmental Policy 21: 55-67.

Rogers, E. M. (1995): Diffusion of Innovations. Free Press, New York.

SCHukAt, S., Theuvsen, L. und H. HeISE (2019): IT in der Landwirtschaft: mit einheitlichen Definitionen zu einheitlichem. In: MEYER-Aurich, A., GANDOrfer, M., BARTA, N., Gronauer, A., KANTELHARDT, J. und H. FLOTO (Hrsg.): Digitalisierung in kleinstrukturierten Regionen - ein Widerspruch in sich? Gesellschaft für Informatik e.V., Bonn: 211-216.

SCHAPER, C., EMmANN, C. H., LINNE, N. und L. THEUvSEN (2013): Stakeholder-Kommunikation in kleineren und mittleren Unternehmen der Bioenergiebranche - Identifizierung, Bewertung und Management relevanter Anspruchsgruppen. In: MEYER, A. (Hrsg.): Kommunikation kleiner und mittlerer Unternehmen, Jahrbuch der KMU-Forschung und -Praxis 2013, Josef Eul Verlag, Köln und Lohmar: 9-28.

SCHLEICHER, S. und M. GRANDORFER (2018): Digitalisierung in der Landwirtschaft: Eine Analyse der Akzeptanzhemmnisse. In: RuCKelshausen, A., MeYer-Aurich, A., BOrCHARDT, K., HOFACKer, C., Loy, J.-P., Schwerdtfeger, R., Sundermeier, H.-H., Floto, H. und B. Theuvsen (Hrsg.): Digitale Marktplätze und Plattformen. Lecture Notes in Informatics 278, Gesellschaft für Informatik, Bonn: 203-206.

SCHumpeter, J. A. (2005): Capitalism, Socialism, and Democracy. Harper \& Row, New York.

SCHWAAB, K. (2016): Die Vierte Industrielle Revolution. Pantheon Verlag, Mehlingen.

SHAPIRO, C. und H. R. VARIAN (1999): Information Rules: A Strategic Guide to the Network Economy. Havard Business Press Books, Boston, MA.

SIMON, H. A. (1956): Rational choice and the structure of the environment. In: Psychological Review 63 (2): 129-138, doi: 10.1037/h0042769.

SPARROW, R. und M. HOWARD (2020): Robots in agriculture: prospects, impacts, ethics, and policy. In: Precision Agriculture 22 (2021): 818-833, doi: 10.1007/s11119-020-09757-9.

Spiller, A., Gauly, M., Balmann, A., Bauhus, J., Birner, R., Bockelmann, W., Christen, O., Entemann, S., Grethe, H., Knierim, U., Latacz-Lohmann, U., Martinez, J., Nieberg, H., QAim, M., Taube, F., Tenhagen, B.-A. und P. Weingarten (2015): Wege zu einer gesellschaftlich akzeptierten Nutztierhaltung. In: Berichte über Landwirtschaft, Special Issue 221: 1-171, doi: 10.12767/buel.v0i221.82.

SPILLER, A., KAYSER, M. und J. BÖHM (2012): Unternehmerische Landwirtschaft zwischen Marktanforderungen und gesellschaftlichen Erwartungen in Deutschland ... aus Sicht der Forschung. In: Schriften der Gesellschaft für Wirtschafts- und Sozialwissenschaften des Landbaues e.V. 47: 11-22.

VOerste, A. (2008): Lebensmittelsicherheit und Wettbewerb in der Distribution. Josef Eul Verlag, Köln und Lohmar.

von Hobe, C.-F., Michels, M. und O. Mußhoff (2021): Präferenz deutscher Landwirte für Pachtpreisanpassungsklauseln unter Berücksichtigung des Klimawandels und der Änderung agrarpolitischer Rahmenbedingungen. In: Berichte über Landwirtschaft 99 (1): 1-19, doi: 10.12767/buel.v99i1.312. 
Woodhouse, P. (2010): Beyond Industrial Agriculture? Some Questions about Farm Size, Productivity and Sustainability. In: Journal of Agrarian Change 10 (3): 437-453, doi: 10.1111/j.1471-0366.2010.00278.x.

ZIMMERMANN, M. (2003): Das Kaufverhalten von Landwirten im Bereich landwirtschaftlicher Investitionsgüter und die Auswirkungen auf den Marketing-Mix landtechnischer Unternehmen. Cuvillier Verlag, Göttingen. 


\section{Teil I:}

\section{Elektromobilität und Einsatz autonomer Feldroboter in der Land- wirtschaft aus Sicht deutscher Landwirte}

\section{I.1 Elektromobilität in der Landwirtschaft - Eine qualitative Analyse zur Nutzerakzeptanz}

E-Mobility in German Agriculture - A Qualitative Analysis of User Acceptance

Friedrich Rübcke von Veltheim, Ansgar A. Rube und Heinke Heise

Geleisteter Eigenanteil an dem Beitrag

\begin{tabular}{|c|c|c|c|c|c|}
\hline $\begin{array}{l}\text { Literatur- } \\
\text { recherche }\end{array}$ & $\begin{array}{l}\text { Entwicklung } \\
\text { des } \\
\text { Forschungs- } \\
\text { rahmens }\end{array}$ & $\begin{array}{l}\text { Konzept und } \\
\text { Design der Stu- } \\
\text { die }\end{array}$ & $\begin{array}{l}\text { Auswahl, Aus- } \\
\text { führung und } \\
\text { Entwicklung } \\
\text { von Methoden }\end{array}$ & $\begin{array}{l}\text { Interpretation } \\
\text { der } \\
\text { Ergebnisse }\end{array}$ & $\begin{array}{l}\text { Konzept und } \\
\text { Schreiben des } \\
\text { Artikels }\end{array}$ \\
\hline $50 \%$ & $50 \%$ & $50 \%$ & $50 \%$ & $60 \%$ & $>75 \%$ \\
\hline
\end{tabular}

Dieser Beitrag ist so oder in ähnlicher Fassung veröffentlicht in der wissenschaftlichen Zeitschrift „, German Journal of Agricultural Economics“ 70 (2021). 


\section{Inhalt}

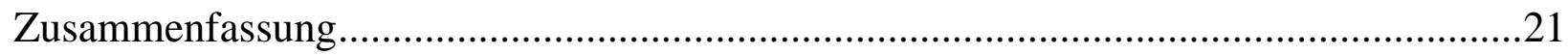

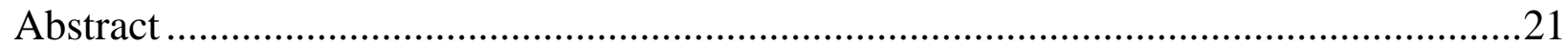

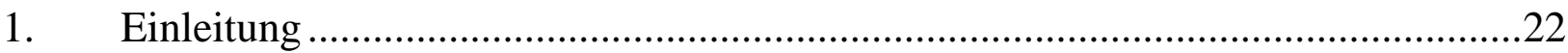

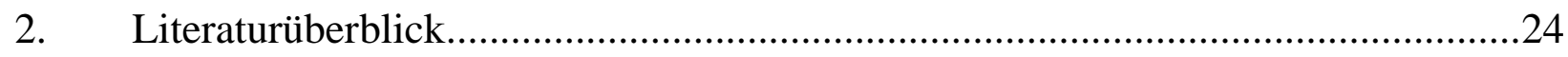

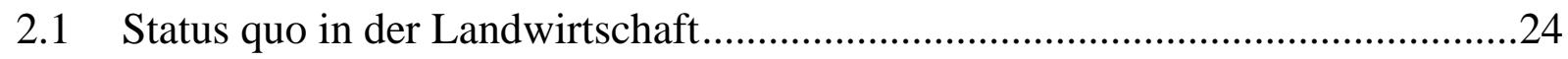

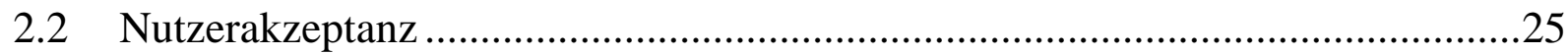

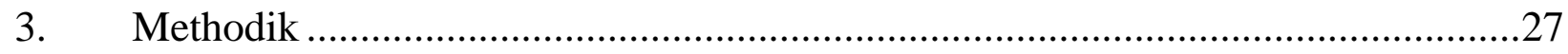

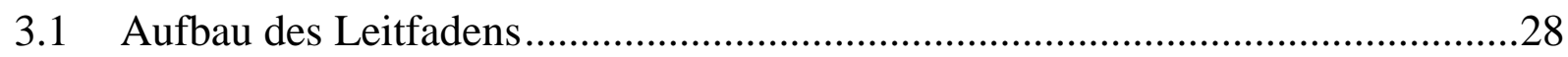

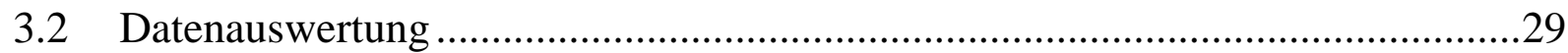

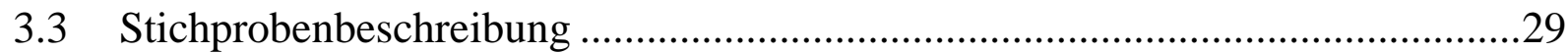

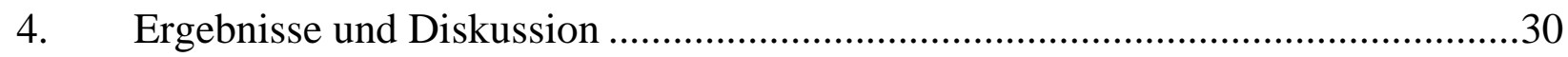

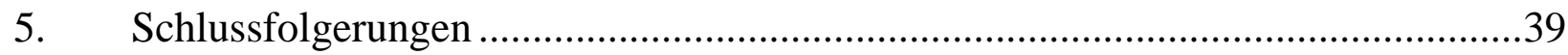

Literatur

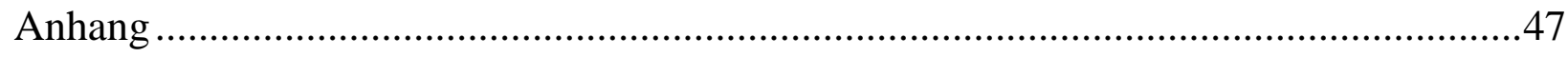




\title{
Zusammenfassung
}

Das Thema E-Mobilität in der Landwirtschaft gewinnt zunehmend an Relevanz, was sich beispielsweise in der Entwicklung erster elektrisch betriebener Traktorenkonzepte manifestiert. Ob sich diese Konzepte allerdings in der Breite etablieren lassen oder als Nische verbleiben, ist noch unklar. Fakt ist, dass die Akzeptanz der E-Mobilität unter Landwirten hierbei künftige eine entscheidende Rolle spielen wird. Über die ex-ante Nutzerakzeptanz elektrisch betriebener Landmaschinen ist, verglichen mit dem Automobilsektor, jedoch noch wenig bekannt. Daher werden in dieser explorativen Studie die Einflussfaktoren der ex-ante Nutzerakzeptanz elektrisch betriebener Landmaschinen qualitativ untersucht, um so einen Beitrag zur weiteren Erforschung der Nutzerakzeptanz zu leisten. Mithilfe qualitativer Experteninterviews wurden 15 Betriebsleiter unterschiedlich organisierter Landwirtschaftsbetriebe zu diesem Thema befragt. Die Ergebnisse verdeutlichen einerseits, dass unter den 15 befragten Landwirten mehrheitlich kritische Positionen bezüglich der Verbreitung und des Einsatzes von E-Mobilität in der Landwirtschaft vorherrschen. Andererseits zeigte sich, dass die ex-ante Nutzerakzeptanz elektrisch betriebener Landmaschinen nicht nur von unterschiedlichen Faktoren beeinflusst wird, sondern dass diese auch untereinander verknüpft und somit teilweise indirekt auf die ex-ante Nutzerakzeptanz der Befragten wirken.

\section{Schlüsselwörter}

deutsche Landwirtschaft, Elektromobilität, Landmaschinen, Nutzerakzeptant, qualitative Experteninterviews, Technologieadoption

\begin{abstract}
The development of battery-powered tractor concepts reveals that the topic of electric mobility (e-mobility) in agriculture is becoming increasingly relevant. However, it is not yet clear whether these concepts can be established on a broad scale or remain as niches. It is a fact that the future acceptance of e-mobility among farmers will play a key role in this context. But compared to the automotive sector, little is known about the ex-ante user acceptance of electrically powered agricultural machinery. Therefore, this explorative study examines the influencing factors of the ex-ante user acceptance of electrically driven agricultural machinery in a qualitative way in order to contribute to further research on user acceptance. With the help of qualitative expert interviews, 15 farm managers of differently
\end{abstract}


organized agricultural farms were interviewed on this topic. It turns out, that the majority of the interviewees are critical in terms of the distribution and use of e-mobility in agriculture. On the other hand, it has been shown that the ex-ante user acceptance of electrically powered agricultural machines is not only influenced by various factors, but that they are also linked to each other and thus have an indirect effect on the ex-ante user acceptance of the respondents.

\section{Keywords}

E-mobility, farm machinery, German agriculture, qualitative expert interviews, technology adaptation, user acceptance

\section{Einleitung}

Von den zunehmend sichtbar werdenden Auswirkungen eines sich ändernden Klimas, die sich beispielsweise in vermehrt auftretenden Extremwetterereignissen äußern, sind Landwirte in besonderer Weise betroffen (GÖMANN et al., 2015). Um die negativen Auswirkungen des Klimawandels zu begrenzen, mehren sich die Forderungen nach einer raschen Dekarbonisierung fossilbasierter Volkswirtschaften, wie Deutschland (HoLTZ et al., 2018; KEMFERT, 2018). Eine Möglichkeit hierzu sieht die Bundesregierung in der Elektrifizierung des bisher auf fossilen Brennstoffen basierten Verkehrssektors, wozu bereits mehrere Maßnahmenpakete geschnürt wurden (BMVI, 2019). Dabei prognostizieren BöSCHE et al. (2017) der E-Mobilität eine breite Marktdurchdringung innerhalb der nächsten Dekade.

Diese Entwicklung wird auch an der Landwirtschaft nicht spurlos vorübergehen, liegen ihr Kohlenstoffdioxidausstoß und Energieverbrauch doch noch weit oberhalb eines als nachhaltig geltenden Niveaus (CAETANO et al., 2017). Auch hat die Bundesregierung im Einklang mit internationalen Vereinbarungen im Rahmen des Klimaschutzplans 2050 deutliche Minderungsziele für die Treibhausgasemissionen verschiedener Wirtschaftssektoren, u.a. der Landwirtschaft, formuliert (BMU, 2019). Aus diesem Grund gewinnt das Thema E-Mobilität in der Landwirtschaft zunehmend an Relevanz, was sich bspw. in der Entwicklung erster elektrisch betriebener Traktorenkonzepte manifestiert (AUMER et al., 2008; JOHN DEERE, 2020). Dennoch ist aufgrund mannigfaltiger Herausforderungen, wie den sehr unterschiedlichen Einsatzbedingungen und einer großen Vielfalt anwendungsbedingter Anforderungen, der Zeitpunkt einer breiten Marktdurchdringung wirtschaftlich effizienter elektrischer Antriebssysteme in der Landwirtschaft - im Gegensatz zur Automobilindustrie - noch nicht absehbar, wenngleich Einigkeit darüber herrscht, dass in Zukunft 
elektrische Antriebe verstärkt Einzug in den landwirtschaftlichen Sektor finden werden (HERLITZIUS, 2018).

Zur größtmöglichen Genese und Diffusion dieser neuen Antriebstechnologie, ist die künftige Akzeptanz verschiedener Akteursgruppen, wie den Nutzern selbst, von entscheidender Bedeutung (KAIRIES, 2013). Die ist neben der Analyse der Einflussfaktoren und Mechanismen, die auf die Technikakzeptanz wirken, erklärtes Ziel der Technikakzeptanzforschung (HÜSING et. al., 2002; KollMANN, 1998; QUIRING, 2006) Der Akzeptanzbegriff umfasst dabei nicht nur das Ausbleiben von Widerstand, sondern auch die aktive Mitwirkung und Handlungsbereitschaft der Akzeptanzsubjekte, in diesem Fall der Landwirte (SCHÄFER und KEPPLER, 2013). Während die Nutzerakzeptanz von E-Mobilität im privaten Sektor bereits in verschiedenen Studien untersucht wurde (vgl. etwa BÜHNE et al., 2015; KruPa et al., 2014; Peters und HoffMAnN, 2011; ReZVANi et al., 2015), ist der landwirtschaftliche Sektor in Bezug auf dieses Thema bislang weitestgehend unerforscht geblieben (RIEDNER et al., 2019). Um diesem Forschungsdefizit zu begegnen, untersucht die vorliegende Studie explorativ die Einflussfaktoren auf die ex-ante Nutzerakzeptanz von elektrisch betriebenen Landmaschinen (eLM). Dazu wurden mithilfe qualitativer Experteninterviews 15 Betriebsleiter unterschiedlich organisierter Landwirtschaftsbetriebe zu diesem Thema befragt.

Nach einem kurzen Literaturüberblick zum Status quo und der Nutzerakzeptanz von eLM (Kapitel 2) folgt eine ausführliche Beschreibung der methodischen Vorgehensweise und des Interviewleitfadens (Kapitel 3), woran sich die Darstellung der wichtigsten Studienergebnisse und deren Diskussion (Kapitel 4) knüpfen. Abschließend werden Schlussfolgerungen gezogen und Handlungsempfehlungen für die Landwirtschaft ausgesprochen (Kapitel 5). Dabei werden die Grenzen der Studie aufgezeigt und weiterer Forschungsbedarf dargelegt. 


\section{Literaturüberblick}

Nachfolgend wird zunächst ein kurzer Überblick über den Ist-Zustand von $\mathrm{eLM}^{7}$ gegeben, bevor auf die bisherigen wissenschaftlichen Erkenntnisse zur Akzeptanzforschung der E-Mobilität und deren Einflussfaktoren eingegangen wird.

\subsection{Status quo in der Landwirtschaft}

Nachdem John Deere bereits im Jahr 2011 ein teilelektrifiziertes Traktorenkonzept vorgestellt hat, wurde im Jahr 2015 mithilfe des Förderprogramms „IKT für Elektromobilität II“ des Bundesministeriums für Wirtschaft und Energie ein vollelektrischer Traktor im sogenannten SESAM-Projekt (Sustainable Energy Supply For Agricultural Machines) entwickelt (STOEHR et al., 2015). Von den beiden verfolgten Ansätzen (mitgeführte Batterie und kabelgebunden) wird die kabelgebundene Variante im Zuge des „GridCon Porjects“ fortgeführt (JOHN DEERE, 2020). Dabei kann der entwickelte Prototyp über zwei wartungsfreie Elektromotoren, von denen der eine die Zapfwelle und das Hydrauliksystem und der andere den Traktor antreibt, bei einer Maximalgeschwindigkeit von $50 \mathrm{~km} / \mathrm{h}$ bis zu $130 \mathrm{~kW}$ Leistung entfalten, wobei eine Batterieladung bis zu vier Betriebsstunden lang hält. Daneben hat auch der Landmaschinenhersteller Fendt zwei eLM Konzepte (e100 Vario und X Concept) entwickelt, wovon der e100 Vario mit einer Leistung von $50 \mathrm{~kW}$ und der X Concept mit bis zu $130 \mathrm{~kW}$ angegeben wird (FENDT, 2020). Dabei ist der X Concept teil- und der e100 vollelektrisiert. Sowohl John Deere also auch Fendt betonen mögliche Einsparungen bei Energie-, Wartungs- und Servicekosten sowie den emissionsfreien und vergleichsweise geräuscharmen Betrieb der eLM als Vorteile gegenüber dieselbetriebenen Landmaschinen.

Neben diesen Konzeptfahrzeugen werden auch schon marktreife Produkte angeboten, wie bspw. von den Firmen Kramer, Siloking und Weidemann. Der Landmaschinenhersteller Kramer hat mit dem KL 25.5e einen vollelektrischen Radlader im Produktportfolio, der ebenfalls auf zwei Elektromotoren (für Fahrantrieb und Hydraulik) setzt und bei einer Ladezeit von fünf bis neun Stunden, drei bis fünf Stunden laufen kann (KRAMER, 2020). Die Firma Weidemann vertreibt den 1160 eHoftrac, der ebenfalls über zwei Elektromotoren, deren Blei-Säure-Batterien über einen 400 Volt Stromanschluss geladen werden, angetrieben wird und bei einer Ladezeit von acht Stunden anderthalb bis vier Stunden Laufzeit vorweist (WEIDEMANN, 2020). Bei denen vom Landmaschinenhersteller Siloking angebotenen Truckline e.0 und Silokamm e.0 handelt es sich um einen vollelektrischen

\footnotetext{
${ }^{7}$ Für eine umfassendere Übersicht zum technischen Stand siehe PICKEL (2019a).
} 
Futtermischwagen und ein vollelektrisches Silageverteilergerät, die ebenfalls mit Blei-Batterien betrieben werden (SILOKING, 2020). Die Einsatzzeit wird mit zwei Betriebsstunden bzw. fünf Mischungen pro Batterieladung angegeben. Daneben werden von den Firmen John Deere, Fendt und Pöttinger auch elektrische Anbaugeräte, wie Schwader präsentiert, während der Landmaschinenhersteller Amazone eine teilelektrische Sämaschine mit Saatgut-Separator entwickelt hat. Andere Beispiele wären eine teilelektrische Ballenpresse von Krone oder ein teilelektrischer Roder der Firma Grimme (AGRARHEUTE, 2017).

Zusammenfassend lässt sich festhalten, dass sich viele eLM noch in der Produktentwicklungs- oder frühen Markteinführungsphase befinden. Dies wird von der Aussage des Landmaschinenherstellers Claas, dass ökonomisch sinnvolle Lösungen noch einige Zeit in ihrer Entwicklung brauchen werden, gestützt. PICKEL (2019b) prognostiziert perspektivische Klarheit in der Entwicklung von eLM bis spätestens Ende der 2020er Jahre und sieht gröBere Potenziale von eLM eher in kleineren, mobilen Landmaschinen mit einem geringeren Leistungsbedarf, idealerweise kombiniert mit eigener Stromerzeugung aus erneuerbaren Energiequellen.

\subsection{Nutzerakzeptanz}

Die Technikakzeptanzforschung beschäftigt sich vor allem mit der Frage: ,„(..) wie einmal eingeführte oder kurz vor der Einführung stehende Techniken von den potenziellen Nutzern, Konsumenten und der allgemeinen Öffentlichkeit aufgenommen, bewertet und eingestuft werden. "(RENN, 2005). Dabei hat das Thema der Nutzerakzeptanz gerade in der gesellschaftspolitischen Diskussion um die Energiewende und damit verbundene neue Antriebstechnologien erneut an Bedeutung gewonnen, da die dafür notwendigen individuellen und gesellschaftlichen Veränderungen nur mithilfe einer breiten Akzeptanz tragfähig sein werden (SCHÄFER und KEPPLER, 2013). Um den derzeitigen Stand der Nutzerakzeptanz von eLM zu umreißen, wird aufgrund des weitgehend unerforschten Bereichs zunächst auf Studien eingegangen, die sich mit Akzeptanzfaktoren von elektrischen Fahrzeugen allgemein beschäftigen ${ }^{8}$ und dann, so vorhanden, ein Bezug zur Landwirtschaft hergestellt.

Für die Akzeptanz von elektrischen Fahrzeugen unter Konsumenten konnte gezeigt werden, dass diese von vielen verschiedenen Faktoren abhängt, die sich entweder akzeptanzhemmend oder -fördernd auf die (potenziellen) Nutzer auswirken. REZVANI et al. (2015) haben im Zuge einer Literaturanalyse die Ergebnisse von 16 empirische Studien zur Nutzerakzeptanz elektrischer Fahrzeuge miteinander vergleichen und die Einflussfaktoren in

\footnotetext{
${ }^{8}$ Für einen umfassenderen Literaturüberblick empfehlen sich die Studien von REZVANI et al. (2015) oder GLOBISCH et al. (2013).
} 
individuelle und soziale Faktoren, technische Faktoren, Kontextfaktoren und Kostenfaktoren unterteilt. Ein Beispiel für einen individuellen Faktor stellt die Einstellung des potenziellen Nutzers zur E-Mobilität dar. So bestätigen EGBUE und LONG (2012) in ihrer Studie zu Unterschieden in der Wahrnehmung und Einstellung zu elektrischen Fahrzeugen, dass technisch versierte Menschen eher dazu neigen Elektrofahrzeuge zu adoptieren. BüHNE et al. (2015) gehen davon aus, dass der Erfolg und die Durchsetzung der E-Mobilität sogar wesentlich von der Einstellung der Nutzer und deren damit einhergehenden Akzeptanz abhängt. Dies konnte gerade in der Landwirtschaft vielfach durch Studien bestätigt werden (u.a. AUBERT et al., 2012; AUSTIN et al., 1998; VOSS et al., 2008). Ein weiterer wichtiger individueller Faktor stellt die Offenheit gegenüber Innovationen dar. HARDMAN et al. (2016) konnten für early-adopters von elektrischen Fahrzeugen nachweisen, dass sich soziodemografische und psychologische Eigenschaften des Nutzers, wie dessen Offenheit gegenüber Innovationen, auf dessen Adoptionsverhalten auswirken. VON JEINSEN et al. (2018) und Aguilar-GaLlegos et al. (2015) konnten belegen, dass die Offenheit für Innovationen aber auch der Kontakt zu Informationen gerade in der Landwirtschaft eine große Rolle im Akzeptanzprozess neuer Technologien spielt. Der Faktor Information in Form des Wissens über die Potenziale von elektrischen Fahrzeugen allgemein wurde in der gesichteten Literatur mehrfach belegt (KAHN, 2007; LANE und POTTER, 2007; MOONS und DE Pelsmacker, 2012; ReZVANi et al., 2015). Dabei führt ein begrenztes Wissen unter den (potenziellen) Nutzern zu negativen Auswirkungen auf das Adoptionsverhalten (BÖSCHE et al., 2017; KAIRIES, 2013; PETERS et al., 2013), was für andere Technologien ebenso in der Landwirtschaft bestätigt wurde (u.a. AUBERT et al., 2012; ERICKSON, 2017; SCHIMMELPFENNIG und EBEL, 2016; THOMPSON et al., 2018). Bei den technischen Faktoren wirken sich besonders die Ladezeit und -kapazität sowie die damit verbundene Reichweite elektrischer Fahrzeuge akzeptanzhemmend aus, da diese vielfach als noch nicht ausreichend empfunden wird, was gleichermaßen für die Landwirtschaft gilt (BÖSCHE et al., 2017; KAIRIES, 2013; Peters und HofFMAnN, 2011). Das für private elektrische Fahrzeuge identifizierte technische Akzeptanzhemmnis unzureichender Ladeinfrastrukturen (PETERS et al., 2013) kommt nach Ansicht von RIEDNER et al. (2019) in der Landwirtschaft weniger zum Tragen, da viele Höfe häufig eigenen Strom produzieren. Ein weiterer technischer Faktor ist die Umweltverträglichkeit elektrischer Fahrzeuge. KRUPA et al. (2014) analysierten Faktoren, die die Adoption von Plug-in-Hybrid-Elektrofahrzeugen beeinflussen, wobei die Reduzierung von Treibhausgasemissionen als ein wichtiger Aspekt bestätigt wurde. Eine positive Wirkung der Umweltverträglichkeit elektrischer Fahrzeuge wurde zusätzlich von EGBUE et al. (2017), GöTZ et al. (2011), GRAHAM-RowE et al. (2012) und PETERS et al. (2011) bestätigt. Zu den wichtigsten Kostenfaktoren bei der Akzeptanz 
elektrischer Fahrzeuge zählen die Anschaffungs- und Betriebskosten (BöSCHE et al., 2017; Peters et al., 2011; Peters und HofFMAnN, 2011; SCHNEIDER et al., 2014; SKIPPON und GARWOOD, 2011). BOZEM et al. (2013) haben in einer Umfrage unter Pkw-Nutzern ermittelt, dass die Kosten für Kauf und Betrieb elektrischer Fahrzeuge für die Befragten erst dann akzeptabel sind, wenn diese auf dem Niveau konventioneller Fahrzeuge sind, allenfalls diese leicht übersteigen und eine Alltags- und Gebrauchstauglichkeit gegeben ist. Als ein maßgeblicher Kontextfaktor zählt die Politik, insofern, als dass sie durch entsprechende Fördermaßnahmen großen Einfluss auf die Nutzerakzeptanz der E-Mobilität ausüben kann (BÜHNE et al., 2015). Eine entsprechende Wirkung auf die Akzeptanz von (potenziellen) Nutzern durch gezielte, politisch herbeigeführte Anreizsysteme bestätigen auch DÜTSCHKE et al. (2013), HEymann (2013), KAINE et al. (2007) und PETERS et al. (2013).

Generell lässt sich ein großes Forschungsdefizit in Bezug auf den ex-ante Akzeptanzprozesses von elektrischen Fahrzeugen in der Landwirtschaft feststellen, obgleich verhältnismäßig viele Studien zum Akzeptanzprozess von elektrischen Fahrzeugen in der Automobilbranche existieren. Vor diesem Hintergrund scheint eine Überprüfung der Übertragbarkeit von Erkenntnissen aus der Automobilbranche auf die Landwirtschaft besonders interessant.

\section{Methodik}

Zur Erhebung der verwendeten Daten wurden Experteninterviews nach MAYRING (2015) mit 15 landwirtschaftlichen Betriebsleitern geführt und im Anschluss qualitativ analysiert (GLÄSER und LAUDEL, 2012). Die Fragen des dabei verwendeten Interviewleitfadens (siehe Anhang) wurden auf Grundlage, der aus der verfügbaren Literatur zu diesem Thema gebildeten Einflussfaktoren formuliert. 


\subsection{Aufbau des Leitfadens}

Ziel einer qualitativen Inhaltsanalyse ist es, die aus einer Kommunikationsart entspringenden Informationen systematisch und regelgeleitet zu analysieren (MAYRING, 2015). Im Unterschied zu quantitativen Befragungen wird eine statistische Repräsentativität nicht primär angestrebt. Vielmehr geht es darum, einen bisher wenig erforschten Sachverhalt zunächst explorativ mithilfe der in den Experteninterviews rekonstruierten Situationen hinsichtlich der Erfahrungen, Meinungen und Einstellungen der befragten Experten empirisch zu analysieren. Auf diese Weise wird ein Fundament für mögliche weiterführende, quantitative Forschungen gelegt (GLÄSER und LAUDEL, 2012; PATTON, 2015). Die qualitative Vorgehensweise wurde in der vorliegenden Studie gewählt, da für das Untersuchungsfeld im landwirtschaftlichen Kontext bisher kaum Daten vorliegen.

Um die Gespräche fokussiert ablaufen zu lassen und die Voraussetzungen zur Erfassung aller für die Fragestellung der Studie relevanten Informationen während der Erhebung zu gewährleisten, wurde im Vorfeld ein Interviewleitfaden formuliert (siehe Anhang). Diesem lagen folgende Leitfragen zu Grunde:

- Welche Ausprägungen der einzelnen Einflussfaktoren sind in der Wahrnehmung der Experten wichtig oder unwichtig bzw. wird ein Vor- oder Nachteil wahrgenommen?

- Wie tragen die Ausprägungen bzw. Einflussfaktoren zur Akzeptanz bei?

- Wie ist die momentane Einstellung der Experten zum Thema der E-Mobilität und einer Adoption der Technologie?

Auf dieser Grundlage wurden 31 Fragen (siehe Anhang) für die halbstandardisierten Experteninterviews aufgestellt, wobei Erkenntnisse von Untersuchungen zur E-Mobilität im Bereich der Automobilindustrie sowie Schlussfolgerungen zur Nutzerakzeptanz von Innovationen in der Landwirtschaft berücksichtigt wurden (AUGENSTEIN, 2015; BOZEM et al., 2013; BÜHNE et al., 2015; FAR und REZAI-MOghAdDAM, 2017; FrANKE et al., 2013; KAINE et al., 2007; KAIRIES, 2013; PETERS und HOFFMANN, 2011; RIEDEMANN, 2011; VON JEINSEN et al., 2018). Es wurde darauf geachtet, die Fragen offen und neutral zu formulieren. Nach einer kurzen Hinführung zum Thema wurden die Experten zu den zuvor aus der Literatur abgeleiteten Einflussfaktoren auf die Akzeptanz von E-Mobilität in der Landwirtschaft befragt. Diese waren im Einzelnen: Offenheit für Innovationen, Information, Technik, Umwelt, Preis, Politik und Einstellung zu E-Mobilität (AltenbURG et al., 2016; AUVINEN et al., 2016; BRUNNENGRÄBER und HAAS, 2018; CANSINO et al., 2018; GNANN und PlÖTZ, 2011; HeYMANN, 2013; KIHM und TROMMER, 2014; MISERIOR, 2018; NPE, 2018; 
PETERS et al., 2013; PICKEL, 2018; YAY, 2012). Abschließend gab es seitens der Experten die Gelegenheit, Ergänzungen vorzunehmen oder Sachverhalte anzusprechen, die nicht Teil des Leitfadens waren.

\subsection{Datenauswertung}

Die aufgenommenen und im Anschluss transkribierten Experteninterviews wurden aufbereitet und die zur Beantwortung der, eingangs gestellten, Frage erforderlichen Informationen mithilfe der Software „f4 Analyse“ extrahiert und nach GLÄSER und LAUDEL (2012) qualitativ analysiert. Dafür wurden die auf diese Weise gewonnenen Daten den eingangs aus der verfügbaren Literatur abgeleiteten Einflussfaktoren auf die Akzeptanz von eLM zugeordnet, wobei eine nachträgliche Ergänzung im Falle neu erfasster, zuvor nicht berücksichtigter Informationen möglich blieb. Dies war insofern wichtig, als dass vorab kaum Erkenntnisse bzw. Zusammenhänge zwischen den akzeptanzbeeinflussenden Faktoren gebildet werden konnten, was wiederum der begrenzten Literatur zu dieser Problemstellung geschuldet war (GLÄSER und LAUDEL, 2012). Zu einer solchen Faktorenneubildung kam es im Laufe der Auswertung allerdings nicht. Aus den 15 Interviews konnten über 600 Textstellen den Einflussfaktoren zugeordnet und auf diese Weise strukturiert werden; dies diente als Grundlage für die Darlegung der Ausprägungen und Beziehungen der untersuchten Faktoren im nachfolgenden Ergebnisteil.

\subsection{Stichprobenbeschreibung}

Für die Interviews wurden 15 landwirtschaftliche Betriebsleiter als Experten ausgewählt, deren demografische und betriebsspezifische Daten der Tabelle A1 (siehe Anhang) entnommen werden können. Als einziges Ausschlusskriterium galt dabei eine Betriebsaufgabe in absehbarer Zukunft. Die befragten Landwirte waren alle männlich und zwischen 24 und 64 Jahre alt. Das Durchschnittsalter liegt bei 41 Jahren. Unter den ausgewählten Experten gaben sechs den Agrarbetriebswirt, fünf ein abgeschlossenes Studium und zwei einen landwirtschaftlichen Meister als höchsten Ausbildungsgrad an. Eine Ausbildung zum Landwirt und keine landwirtschaftliche Ausbildung wurden je einmal genannt. Fast alle der von den Experten geführten Betriebe befinden sich in Niedersachsen; lediglich einer in Mecklenburg-Vorpommern. 12 der Betriebe werden im Haupt- und drei im Nebenerwerb betrieben; 10 Betriebe sind reine Ackerbaubetriebe, während die restlichen vier Betriebe gemischt (drei Mal Ackerbau und Milchvieh, einmal Ackerbau und Schweinemast) wirtschaften. Ein Betrieb ist darüber hinaus Direktvermarkter. Die Flächenausstattung variiert zwischen 30 und 1.000 Hektar (ha) bewirtschaftete Fläche; im Mittel liegt die bewirtschaftete Fläche bei 239,4 ha. 


\section{Ergebnisse und Diskussion}

Nachfolgend werden zunächst die Ergebnisse der untersuchten Akzeptanzfaktoren chronologisch vorgestellt und jeweils anschließend diskutiert. Abschließend wird aus den gewonnenen Erkenntnissen ein Modell abgeleitet und dargestellt.

Für den Einflussfaktor Offenheit für Innovationen konnte durch die Auswertung der Interviews gezeigt werden, dass dieser für die künftige Adoption von E-Mobilität in der Landwirtschaft wichtig ist. Da in dieser Untersuchung keine sich dem Thema der E-Mobilität in der Landwirtschaft gänzlich verschließende Gruppe ausgemacht werden konnte, kann von einem grundlegenden Interesse der Landwirte an dieser Technologie gesprochen werden. Unter den befragten Landwirten wurden drei verschiedene Gruppen identifiziert: eine aufgeschlossene, eine abwartende und eine rational abwägende Gruppe (siehe Tabelle A2 im Anhang).

Die aufgeschlossene Gruppe sieht Offenheit für Innovationen, auch in Bezug auf eLM, als wichtig für die Weiterentwicklung und Zukunftsfähigkeit des eigenen landwirtschaftlichen Betriebes an. Daneben ist für die Experten dieser Gruppe Offenheit für Innovationen die Voraussetzung für einen besseren Informationsstand, der einen Wettbewerbsvorteil gegenüber weniger informierten Betriebsleitern darstellt (Landwirte 1, 2, 6 und 12).

„Das ist schon eine wichtige Voraussetzung, um letztendlich auch für den Alltag gut informiert zu sein, damit man auch Entscheidungen [...] ausführen kann." (Landwirt 12)

Es konnte somit ein Einfluss des Faktors Offenheit für Innovationen auf den ebenfalls aus der Literatur gewonnen Faktor Information festgestellt werden.

Zur abwartenden Gruppe sind Experten zusammengefasst, die sich durch Zurückhaltung im Hinblick auf E-Mobilität in der Landwirtschaft auszeichnen. Technische Entwicklungen werden abwartend aufgenommen; eine Adoption zu einem späteren Zeitpunkt wird aber nicht ausgeschlossen. Die Befragten sehen für ihren Betrieb mittelfristig keinen Schwerpunkt in der E-Mobilität (Landwirte 3, 9 und 10).

Die größte Gruppe (acht der 15 Experten) ist durch Aussagen charakterisiert, die Offenheit für Innovationen vor allem mit Kosten und Nutzen einer neuen Technologie in Verbindung bringen. Diese rational abwägende Gruppe macht ihre Offenheit von ihren jeweiligen betrieblichen Strukturen bzw. Einsatzbereichen abhängig und kommt zu dem Schluss, dass sie sich nicht als „Vorreiter“ neuer Technologien bezeichnen würde; eine wahrgenommene ökonomische Vorteilhaftigkeit und die Möglichkeit, eLM testen zu können, würde ihre 
Offenheit aber erhöhen und eine Adoption der Technologie wahrscheinlicher machen (Landwirte 4, 5, 7, 8, 11, 13, 14 und 15). Hier lässt sich ein Zusammenhang zwischen den Faktoren Preis und Offenheit sowie Offenheit und dem Zeitpunkt der Adoption feststellen. Diese Erkenntnis deckt sich mit dem von AGUILAR-GALLEGOS et al. (2015) identifizierten Einfluss von Offenheit auf die Wahrnehmung von Informationen und auf das Interesse an Innovationen.

Für den Faktor Information wurde eruiert, wie die befragten Betriebsleiter mit verfügbaren Informationen in Kontakt treten, wie ihr Informationsstand ist und welche Informationen für die Befragten wichtig für die Bewertung von E-Mobilität in der Landwirtschaft sind (siehe Tabelle A3 im Anhang).

Der Kontakt zu Informationen ist vielfach wenig vorhanden und wird infolgedessen von den Experten als negativ bewertet (Landwirte 2, 5, 10, 13, 14 und 15). Übereinstimmend dazu gelangten auch BÖSCHE et al. (2017) zu der Erkenntnis, dass sich ein zu geringer Kontakt mit Informationen zur E-Mobilität negativ auf die Nutzerakzeptanz auswirken kann. Als Hauptinformationsquellen wurden Messen und Fachzeitschriften genannt (Landwirte 1, 3-6, 8-13 und 14), wobei unter den Befragten Uneinigkeit hinsichtlich der Gewichtung der benannten Quellen besteht. Die aktive Informationsbeschaffung, die für Messen charakteristisch ist, setzt ein stärkeres Interesse an eLM voraus als dies die Lektüre von Fachzeitschriften erfordert. Insofern zeigt sich ein Zusammenhang zwischen den Faktoren Information und Offenheit für Innovationen. Dieser bestätigt sich auch bei der Frage, wie gut sich die Befragten über eLM informiert fühlen und wie es um ihren derzeitigen Informationsstand bestellt ist. So könnte dieser nach Ansicht aller befragten Experten besser sein. Besteht wenig Interesse an dem Thema, erfolgt keine aktive Aneignung neuer Informationen bzw. neuen Wissens (Landwirte 3, 10 und 15).

„Soweit wie es mich interessiert, ja. Also ich könnte besser informiert sein, aber das Interesse ist nicht da. "(Landwirt 3)

Darüber hinaus sind die angebotenen Informationen zur E-Mobilität nach Aussage einiger Experten im Vergleich mit Informationen über Dieselaggregate nicht umfassend genug (Landwirte 2, 5, 12 und 13). Zu einem vergleichbaren Ergebnis kamen auch BOZEM et al. (2013), indem sie belegten, dass sich Kunden im direkten Vergleich der Antriebskonzepte über konventionelle Fahrzeuge informierter fühlen als über elektrisch angetriebene. Ein zu geringer Kenntnisstand kann sich infolge von Fehleinschätzungen hemmend auf die Nutzerakzeptanz auswirken (KAIRIES, 2013).

Mit Blick auf die Informationsrelevanz für die befragten Experten fordern diese geschlossen die bessere Darlegung eines Gesamtkonzepts und des Praxisbezugs von eLM. 
Adressaten dieser Forderung sind vornehmlich entsprechende Leitmedien und die Maschinenhersteller. Ergänzend dazu betrachten einige Experten Kosten-Nutzen-Berichte, die allen voran technische Aspekte und Kaufpreise in den Vordergrund stellen, als wichtige Informationen (Landwirte 1, 5, 7 und 9). Auch das selbstständige Erproben von eLM und die daraus resultierende Praxiserfahrung bzw. die durch entsprechende Medien berichtete Erfahrung anderer Berufskollegen, gilt vielen Befragten als Quelle von Informationen, die für ihr jeweiliges Einsatzfeld relevant sind (Landwirte 1, 4-8, 10, 12, 13 und 14). Diese wichtigen Informationen bilden nach Ansicht der Experten teilweise die Voraussetzung für den Kauf einer eLM (Landwirte 1 und 5). Damit folgen sie in ihrer Einschätzung den Ergebnissen von PETERS et al. (2013), die bestätigen, dass das Ausprobieren elektrischer Fahrzeuge einer Nutzerakzeptanz dienlich ist. Die so gewonnenen Erfahrungswerte können die Benutzerfreundlichkeit und Einfachheit einer Technologie vermitteln (PIERPAOLI et al., 2013).

Der Faktor Technik umfasst die Merkmalsausprägungen Vor- und Nachteile, landwirtschaftliche Anwendungsbereiche und Ladeinfrastruktur bzw. -zyklen (siehe Tabelle A4 im Anhang).

Als technischen Vorteil von eLM sehen einige Befragte eine stärkere Leistung, eine verbesserte Kraftübertragung, weniger Energieverluste und eine höhere Energieeffizienz (Landwirte 2, 7, 8 und 15). Ebenso werden die geringere Wartung von eLM und ihr vergleichsweise einfacher Aufbau als technisch vorteilhaft gegenüber herkömmlichen, mit Verbrennungsmotoren betriebenen Landmaschinen wahrgenommen (Landwirte 5-8, 11, 12, 14 und 15). Zwei Betriebsleiter merken auch den eingesparten Kraftstoffverbrauch als positiv an (Landwirte 1 und 13). Besonders begrüßen einige der Befragten die sich durch eLM bietenden Möglichkeiten, wie die optimale Einstellung, den niedrigeren Wirkungsverlust und die bessere Steuer- bzw. Regelbarkeit von Anbaugeräten (Landwirte 2, 3, 8 und 12). Die elektrische Steuerung auf 12-Volt-Basis ist bereits möglich und so konnten die Landwirte 3 und 8 auf positive Erfahrungen mit solchen Systemen zurückblicken. Der in den Aussagen zur besseren Steuerbarkeit der Anbaugeräte wahrgenommene Vorteil wirkt sich positiv auf die Einstellung der Landwirte gegenüber eLM aus, worin ein Zusammenhang zwischen den Faktoren Technik und Einstellung zu E-Mobilität ersichtlich wird. So sind es gerade die empfundenen Vorteile, die beim Faktor Technik eine Akzeptanzsteigerung bewirken können (FAR und REZAEI-MOGHADDAM, 2017). Der dazukommende, von Landwirt 14 (Testbetrieb eines Prototyps) vorgenommene Vergleich von Kosten und Nutzen einer Technologie fließt mit in die Bewertung der selbigen ein und kann sich im 
Falle einer wahrgenommenen technologischen Überlegenheit ebenfalls positiv auf die Nutzerakzeptanz auswirken (AGUILAR-GALLEGOS et al., 2015; VON JEINSEN et al., 2018).

Als Hauptkritikpunkte bzw. derzeit bestehende Nachteile der Technologie werden neben der noch unzureichenden technischen Reife der fehlende Kontakt zu Werkstätten, mangelndes Knowhow seitens der Anwender sowie die geringen Reichweiten bzw. nicht ausreichende Energiedichte der Akkumulatoren angeführt (Landwirte 3, 6, 8, 9, 12, 13, 14 und $15)$.

„,Aber die Technik ist noch nicht ausgereift genug, um das selber ganz konkret jetzt für die eigene Planung im Blick zu haben. "(Landwirt 6)

So fordern einige der befragten Experten eine Mindesteinsatzzeit der Landmaschinen zwischen 8 und 14 Stunden (Landwirte 2, 3, 5 und 12). Eine zu geringe Reichweite bzw. Einsatzzeit der Maschinen wurde auch von PICKEL (2018) als größtes bestehendes technisches Akzeptanzhemmnis ausgemacht.

,[...] im Dauerbetrieb hast, dann ist das was anderes. Aber ich denke die normalen Zyklen, da kann man sich darauf einstellen. " (Landwirt 3)

Entscheidende Verbesserungen auf diesem Gebiet sind nach HEYMANN (2013) und NPE (2018) allerdings erst ab Mitte der 2020er Jahre zu erwarten. Dies gilt im Besonderen für Nutzfahrzeuge, da sich diese in hergestellter Stückzahl und Anforderungsprofil wesentlich von Personenkraftwagen unterscheiden (GRÄBENER, 2017). Die genannten Nachteile verringern den wahrgenommenen Nutzen und wirken sich negativ auf die Akzeptanz von E-Mobilität aus. Bezogen auf die landwirtschaftlichen Anwendungsbereiche von eLM sind einigen befragten Landwirten ein vergleichbar breites Einsatzspektrum und eine ähnliche Flexibilität wie beim Einsatz herkömmlicher Landmaschinen wichtig (Landwirte 1 und 5). Dies unterstreicht die Beobachtung von KAINE et al. (2007), dass Landwirte vor allem ein breites Einsatzspektrum sowie eine hohe Flexibilität bei technischen Innovationen schätzen. Auch die Forderung einiger Befragter, dass eLM leistungstechnisch ein Äquivalent zu konventionellen Maschinen darstellen sollen (Landwirte 1, 5 und 6), wird in der Literatur bestätigt (BOZEM et al., 2013). Insgesamt erscheint die Bodenbearbeitung beim Ackerbau einigen Experten als ein wenig geeigneter Einsatzbereich von eLM (Landwirte 1, 7, 8, 12 und 13). Sie sehen das Potenzial eher im Bereich von Pflegemaßnahmen, wie dem Ausbringen von Dünger, Saatgut und Pflanzenschutzmitteln (Landwirte 1, 2, 7, 9, 11, 12 und 14). Einige der Befragten können sich angesichts des derzeitigen Entwicklungsstandes von eLM den Einsatz vorzugsweise in viehhaltenden Betrieben vorstellen, da hier die Einsatzzeiten der Maschinen vergleichsweise kurz sind und diese anschließend wieder geladen werden könnten (Landwirte 1 und 2). Darüber hinaus ist der emissionsfreie Betrieb 
elektrischer Geräte in geschlossenen Räumen, wie Ställen, vorteilhafter als der Einsatz abgaserzeugender, mit Verbrennungsmotoren betriebener Maschinen (Landwirte 1, 8 und 11). Hier lässt sich ein Zusammenhang zwischen den Faktoren Umwelt und Technik erkennen. Das von PETERS und HOFFMANN (2011) angeführte Argument, elektrische Fahrzeuge könnten als Erweiterung konventioneller Landmaschinen gesehen werden, konnte nicht bestätigt werden. Vielmehr ist einigen Befragten eine flexibel einsetzbare Maschine wichtig, was mit der allgemein schwierigen wirtschaftlichen Lage vieler Betriebe und ihrer daraus resultierenden beschränkten Investitionsfähigkeit erklärt werden könnte (Landwirte 1 und 3). So kann die Investition in ein vielseitig einsetzbares Gerät wirtschaftlich attraktiver sein als die Investition in eine bloße Erweiterung bestehender Technik.

Die meisten Landwirte bewerten die Umsetzbarkeit der Ladeinfrastruktur als weitestgehend unproblematisch, da sowohl Stellplätze als auch Stromanschlüsse auf den Hofstellen vorhanden sind (Landwirte 1-5, 7-10, 12-14 und 15). Es besteht lediglich bei zwei Befragten Unsicherheit bezüglich der Kosten einer passenden Ladestation (Landwirte 6 und 10), was verdeutlicht, dass bei einigen Landwirten der Faktor Information auf den Faktor Technik insofern wirkt, als dass eine Ermangelung an Informationen zu Unsicherheiten in der Bewertung der technischen Umsetzbarkeit führt. Daneben wird von denselben zwei Experten die für schnelle Ladevorgänge benötigte Leistungsfähigkeit des vorhandenen Stromnetzes infrage gestellt. Diese Sorge scheint begründet zu sein, da bspw. STÖHR und HACKENBERG in ihrer 2018 veröffentlichten Untersuchung zu dem Ergebnis kommen, dass die Energie, die für das Laden elektrisch betriebener Traktoren notwendig ist, die Kapazität der bestehenden Stromnetze übersteigt. Einige der befragten Landwirte bemängeln, dass neben dem noch unzureichend ausgebauten E-Tankstellennetz, das für weiter von der Hofstelle entfernte Schläge wichtig wäre, das Ladungsmanagement in Arbeitsspitzen, unter Zeitdruck oder bei unvorteilhaften Witterungsbedingungen noch nicht mit dem dieselbetriebener Landmaschinen mithalten kann (Landwirte 1 und 13). Generell bewerten die Experten das Verhältnis von Einsatz- zu Ladezeiten bei dem derzeitigen Entwicklungsstand von eLM als kritisch (Landwirte 2, 6, 7, 9, 11 und 13). Die dabei immer wieder vorgenommenen Vergleiche mit der technischen Reife elektrisch angetriebener Personenkraftwagen bestätigen die aus der Literatur gewonnene Erkenntnis, dass Menschen neue Mobilitätsmuster oft mit ihnen bereits bekannten vergleichen (PETERS und DÜTSCHKE, 2010).

Auch für den Faktor Umwelt ergaben sich aus den Interviews verschiedene Vor- und Nachteile von eLM, unterschiedliche Sichtweisen auf das Image der Landwirtschaft und Beziehungen zu anderen Faktoren (siehe Tabelle A5 im Anhang). 
Der emissionsfreie und lärmreduzierte Betrieb von eLM wird von vielen der befragten Experten als ökologisch vorteilhaft bewertet (Landwirte 1, 2, 6, 7 und 9). Dies stellt sich in der Automobilbranche ähnlich dar und wirkt sich durch seine positive Wirkung auf den wahrgenommenen Nutzen akzeptanzsteigernd aus (KAIRIES, 2013). Als weitere Vorteile zugunsten der Umwelt werden die Unabhängigkeit von fossilem Kraftstoff und die ressourcenschonendere Nutzung (auch eigenerzeugten) Stroms aus erneuerbaren Energiequellen genannt (Landwirte 1, 3, 5-8, 10, 12 und 13). Ergänzend führt Landwirt 14 aus, dass elektrische Anbaugeräte bspw. eine genauere Applikation von Dünger oder anderen Betriebsmitteln ermöglichen und so einer Verschwendung oder Übernutzung von Ressourcen entgegenwirken, was der Umwelt zugutekommt. Es besteht demnach ein Zusammenhang zwischen den Faktoren Technik und Umwelt. Dennoch hinterfragen viele der Experten die genannten Vorteile auch kritisch, namentlich wegen vorhandener Unklarheiten bezüglich der Umweltbilanzen von eLM und möglicher Weiterentwicklungen konventioneller Technik (Landwirte 1, 2, 5, 8 und 9).

„, Wenn du jetzt die Batterie herstellst oder alles andere dazu herstellst, wie lange hättest du dann einen Dieselmotor - eine Technik, die auch sehr voran ist - nutzen können?" (Landwirt 5)

Nach KIHM und TROMMER (2014) kommt es durch Verbesserungen infolge technischer Weiterentwicklungen konventioneller Verbrennungsmotoren oft zu einer Relativierung der Umweltvorteile elektrisch angetriebener Fahrzeuge. Folglich ist diese Kritik nicht unbegründet. Daneben wird besonders die Belastung der Umweltbilanz durch den Herstellungsprozess der Akkumulatoren von mehreren Landwirten als ökologisch nachteilig gesehen (Landwirte 1, 2, 6 und 13). Die tatsächlichen ökologischen Auswirkungen des Herstellungsprozesses der Akkumulatoren sind nach YAY (2012) jedoch schwer abzuschätzen. Auch die Erzeugung regenerativer Energien betrachten einige der Experten bspw. aufgrund der erforderlichen Herstellung von Photovoltaikmodulen oder der Energiebilanz von Biogasanlagen als bedenklich für die Umwelt (Landwirte 10, 11 und 14).

Die E-Mobilität kann nach Ansicht von Landwirt 8 einen positiven Einfluss auf das Image der Landwirtschaft haben, da seiner Meinung nach, die Öffentlichkeit umweltfreundlichere Landmaschinen und weniger Belastung für die Natur fordert. Ergänzend kommen die verringerten Lärm- und Schadstoffemissionen bei der Bearbeitung von Ackerflächen, die sich in einer räumlichen Nähe zu Wohngebieten befinden, dem sozialen Frieden und damit einem positiveren Image der Landwirtschaft entgegen (Landwirte 3 und 5). Das kann sich wiederum akzeptanzsteigernd auf die Landwirte auswirken. Dafür müsse aber das Gesamtkonzept der E-Mobilität in der Landwirtschaft öffentlich besser dargestellt werden, da nach 
Ansicht vieler Befragter derzeit Themen wie Tierhaltung, Pflanzenschutz und Düngung die gesellschaftliche Diskussion um die Landwirtschaft dominieren (Landwirte 1, 2, 8, 13 und 14). Es lassen sich für die Nutzung eLM aufgrund ihrer Umweltvorteile zwei Gruppen identifizieren. Die erste Gruppe ist bereit, für die wahrgenommenen Umweltvorteile einen höheren Preis zu zahlen (Landwirte 6 und 9), was einen Zusammenhang zwischen den Faktoren Umwelt und Preis bestätigt. Für die zweite Gruppe wiegen ökonomische Argumente weit schwerer als ökologische (Landwirte 3, 5, 7, 11, 12 und 15), womit die beschriebene Beziehung sowohl positiv als auch negativ auf den Faktor Preis wirken kann.

„Auf einer Begeisterung und Umweltfreundlichkeit kann man einen Betrieb nicht zukunftsträchtig führen. " (Landwirt 7)

Für den Faktor Preis ergab sich, dass die Landwirte 6 und 14 durchaus bereit wären, einen höheren Kaufpreis für eLM zu zahlen, sofern dieser sich innerhalb eines gewissen Rahmens bewegt (siehe Tabelle A6 im Anhang). Dabei bezieht sich Landwirt 6 auf die Betriebsstunden, die maximal 5 bis 10 Euro über den aktuellen Kosten liegen dürften. Landwirt 7 wäre hingegen nur dann bereit mehr von eLM zu zahlen, wenn die Leistung, verglichen mit konventionellen Landmaschinen, ebenbürtig oder besser ist. Generell war eher keine Mehrzahlungsbereitschaft unter den befragten Landwirten zu vernehmen, was im Einklang mit den Ergebnissen von LONG et al. (2016) steht, die in den gerade zum Beginn der Markteinführung neuer Technologien tendenziell höheren Preisen ein bedeutendes Akzeptanzhemmnis sehen.

„, Wie viel teurer ist die Anschaffung und wie teuer ist die Unterhaltung im Vergleich zu konventionell angetriebenen Maschinen?" (Landwirt 2)

Laut Meinung einiger Experten fließen neben der Höhe des Preises auch eine Reihe anderer Bewertungskriterien, wie die technische Leistung, das Verhalten landwirtschaftlicher Konkurrenten, Investitionskosten, Liquidität des Betriebes oder die Betriebskosten von eLM, in die tatsächliche Kaufentscheidung mit ein (Landwirte 1, 2, 5, 10, 11 und 12). Zu dem Schluss, dass der Preis nicht das alleinige Bewertungskriterium darstellt, kamen auch PETERS et al. (2013) in ihrer Untersuchung. Durch die von mehreren Landwirten aufgestellte Forderung, dass die Leistungsfähigkeit von eLM mindestens der von herkömmlichen Maschinen entsprechen müsse (Landwirte 2, 7 und 10), um eine Mehrzahlungsbereitschaft unter Landwirten zu erzeugen, kann zusammen mit den befürchteten Unterhaltskosten von eLM eine Beziehung zwischen den Faktoren Technik und Preis abgeleitet werden. Dies bestätigt die Ergebnisse von BOZEM et al. (2013). Daneben können sich äußere Einflüsse, wie fehlende Einnahmen bzw. eine angespannte Marktsituation, negativ auf die 
Inkaufnahme höherer Preise auswirken (Landwirte 1, 3, 6 und 13). Eine andere Möglichkeit zur Steigerung der ökonomischen Attraktivität von eLM kann in betriebsspezifischen Gegebenheiten, wie die Verwertung eigenproduzierten Stroms liegen. Dies ist vor allem der Fall, wenn die Kosten des eigenverbrauchten Stroms unter den Kosten des Strombezugs aus dem öffentlichen Netz liegen (STOEHR et al., 2015). Darüber hinaus bestünde die Möglichkeit, Synergieeffekte zu nutzen und bspw. durch die Integration eines Vehicle-toGrid (V2G) Konzepts einen neuen, ökonomisch vorteilhaften Betriebszweig zu etablieren (PICKEL, 2018). Beim V2G-Konzept wird elektrischer Strom von den Fahrzeugakkumulatoren zurück in das öffentliche Stromnetz gespeist, um so eine intelligente Sektorenkopplung zu ermöglichen. Diese Wirkung betrieblicher Einflussgrößen sowohl auf die Wahrnehmung des Preises (Landwirt 4) als auch auf die Nutzerakzeptanz konnte ebenso von CAVALlO et al. (2014) belegt werden und kann je nach Situation positiv oder negativ ausfallen (KAIRIES, 2013).

Der Faktor Politik wird von den Experten sehr unterschiedlich wahrgenommen und bewertet. Die Ergebnisse lassen sich grob in die momentane Wahrnehmung politischer Entscheidungen und die Rolle der Politik für die künftige Verbreitung von E-Mobilität in der Landwirtschaft unterteilen (siehe Tabelle A7 im Anhang).

Aktuell sehen einige Befragte keinen direkten politischen Schwerpunkt auf der Elektrifizierung des Antriebes von Landmaschinen; jedoch indirekt über die Förderung von E-Mobilität im Automobilsektor (Landwirte 6 und 9). Zwar spiele die Politik eine wichtige Rolle für die Verbreitung von eLM (Landwirt 8), doch nimmt sie diese nach Ansicht von Landwirt 10 nur sporadisch und wenn, dann sehr überstürzt wahr. Dies führt vermehrt zu Planungsunsicherheiten für die Landwirte (Landwirte 1 und 4). Mehrere Experten sind der Auffassung, dass politische Entscheidungsträger künftig an einem überzeugenderen Gesamtkonzept für die E-Mobilität in der Landwirtschaft arbeiten sollten, das auch den Ausbau der Infrastruktur zur Sicherung der Stromversorgung beinhaltet (Landwirte 1, 3 und 4). Der Einfluss politscher Entscheidungen auf die Bewertung technischer Innovationen lässt sich auch in der Literatur finden (KAINE et al., 2007). Besonders positiv werden von vielen Befragten mögliche Kaufanreize, wie bspw. staatliche Förderprogramme, als Instrumente zur Förderung der Verbreitung von eLM bewertet (Landwirte 2, 4, 7, 8, 12 und 15).

,[...] Fördermaßnahmen dafür, da ist die Politik natürlich relevant. Das wäre in Richtung Preis, wenn die Maschinen mehr kosten, aber sie auch gefördert werden, dann würde sich das auch wieder ausgleichen " (Landwirt 8) 
Das bestätigt auch HEYMANN (2013) mit seiner Beobachtung, dass sich finanzielle Fördermaßnahmen besser auf die Nutzerakzeptanz auswirken als nicht-finanzielle Anreize. Folglich besteht ein Zusammenhang zwischen den Faktoren Politik und Preis.

Die Ergebnisse zum Faktor Einstellung zu E-Mobilität lassen sich untergliedern in die aktuelle Einstellung zu eLM, Erfahrungen mit sowie das Meinungsbild zum Umstieg auf eLM. Sie bestätigen darüber hinaus, dass Zusammenhänge mit verschiedenen anderen Faktoren bestehen (siehe Tabelle A8 im Anhang).

Befragte mit einer positiven Einstellung gegenüber E-Mobilität unterstreichen aktuell das Erreichen der nötigen technischen Reife und die Finanzierbarkeit als Grundvoraussetzungen für den künftigen Einsatz solcher Maschinen (Landwirte 1, 3 und 6). Daneben wurde auch die Bedeutung der Forschung auf diesem Gebiet hervorgehoben (Landwirt 12).

„Hier muss aber auf der einen Seite finanzierbar sein und zum anderen auch in ähnlicher Weise die Funktion erfüllen wie die herkömmlichen Antriebsaggregate. Wenn das gewährleistet ist, dann wäre sicherlich ein gangbarer Weg. [...] Aber für mich ist da noch sehr viel Forschungsarbeit notwendig. " (Landwirt 12)

Hier lässt sich ein Einfluss des Faktors Technik auf den Faktor Einstellung identifizieren. Dagegen sehen Befragte mit einer negativen Einstellung gegenüber E-Mobilität sowohl die diesbezügliche Politik als auch die durch die Produktion und Entsorgung von Akkumulatoren entstehenden Umweltprobleme kritisch (Landwirte 5, 7, 9 und 13). Es kommt in der Ursachendimension zu einer negativen Auswirkung der Faktoren Politik und Umwelt auf den Faktor Einstellung. Weiterhin berichteten mehrere Landwirte, dass der Informationsgewinn durch die mit eLM gesammelten Erfahrungen eine Bewertung von eLM erleichtert (Landwirte 3, 6, 8 und 12). Der Faktor Information kann hier als ursächlich für den Einfluss auf die Einstellung zu eLM ausgemacht werden. Bezogen auf einen möglichen Umstieg auf eLM nehmen einige Experten eine abwartende Haltung ein (Landwirte 10 und 11). Als Ursache werden Unsicherheiten hinsichtlich der technischen Reife und die noch als mangelhaft wahrgenommene Eignung für die jeweiligen betriebsindividuellen Einsatzbereiche aufgeführt (Landwirte 1, 3, 6, 8, 10-12 und 13).

Die interpretierten Beziehungen der untersuchten Einflussfaktoren sind zusammenfassend in Abbildung 1 dargestellt, wobei die Pfeile die jeweilige Wirkungsrichtung anzeigen. 


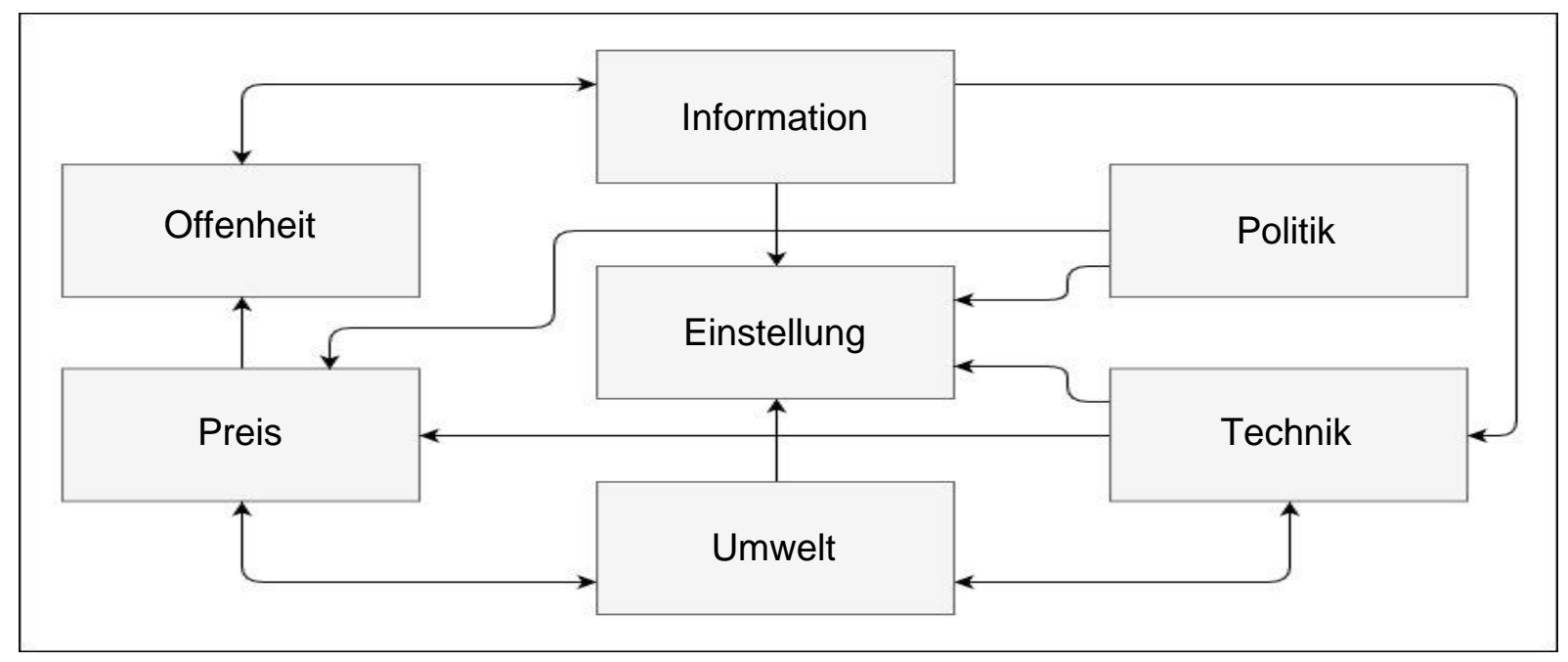

Abbildung 1: Ermittelte Beziehungen zwischen den Einflussfaktoren

Quelle: Eigene Darstellung

\section{Schlussfolgerungen}

Ziel dieser Untersuchung war es, Einflussfaktoren auf die Akzeptanz von eLM mithilfe qualitativer Experteninterviews zu erfassen. Die Ergebnisse zeigen einerseits, dass unter den 15 befragten Landwirten mehrheitlich kritische Positionen bezüglich der Verbreitung und des Einsatzes von E-Mobilität in der Landwirtschaft vorherrschen. Andererseits wird deutlich, dass die Einflussfaktoren in unterschiedlichen Beziehungen zueinanderstehen und dadurch auch mittelbar auf die Wahrnehmung der Befragten wirken. Im Folgenden werden anhand der einzelnen Einflussfaktoren Schlussfolgerungen gezogen und Handlungsempfehlungen abgeleitet.

Die Ergebnisse haben verschiedene Implikationen. Die empirischen Ergebnisse bezüglich des Faktors Offenheit für Innovationen erlauben es Maschinenherstellern und der Fachpresse, die Informationen über E-Mobilität in der Landwirtschaft künftig mehr an die jeweilige Zielgruppe anzupassen, indem sie auf die von den Landwirten geäußerten Bedenken besser eingehen, sofern sie ein Interesse an der Weiterentwicklung und Verbreitung von eLM haben. Ergänzend könnten zwecks Akzeptanzsteigerung mögliche Einsatzbereiche und in einer späteren Markteinführungsphase auch Finanzierungsmöglichkeiten von eLM transparenter kommuniziert werden.

Bezogen auf den Faktor Information könnte unter dem Gesichtspunkt einer Akzeptanzsteigerung von E-Mobilität in der Landwirtschaft ein Ausbau des Informationsangebots unter Beibehaltung der von den Landwirten favorisierten Medien sinnvoll sein. Im Zuge dessen erscheint es ratsam, den praktischen Nutzen der Technologie besser aufzuzeigen und diesen den Landwirten zu vermitteln. Hierfür könnte die Darlegung des von den Befragten 
geforderten verbesserten Gesamtkonzepts zur E-Mobilität in der Landwirtschaft ein Ansatzpunkt sein. Daneben empfiehlt es sich für die Landmaschinenhersteller und -händler, frühzeitig Testmöglichkeiten für eLM anzubieten, um eine Steigerung der Nutzerakzep$\operatorname{tanz}$ zu bewirken. Ergänzend dazu könnten den Landwirten entsprechende Nutzungskonzepte unterbreitet werden, die wahrgenommenen Umstellungsrisiken entgegenwirken.

Um beim Faktor Technik den Forderungen der Befragten zu entsprechen, müssten zunächst die Akkumulatoren der eLM hinsichtlich ihrer Laufzeit weiterentwickelt werden. Ob sich darüber hinaus ein Netzausbau positiv auf die Nutzerakzeptanz auswirken würde, bleibt abzuwarten, da die Leistungsfähigkeit der Stromnetze nur von wenigen Landwirten als Problem wahrgenommen wurde und oftmals eine hofeigene Stromproduktion aus regenerativen Energiequellen betrieben wird.

Für den Faktor Umwelt zeigt sich, dass sich E-Mobilität in der Landwirtschaft durch ihr umweltfreundliches Image positiv auf die Nutzerakzeptanz auswirkt. Damit diese und das dazugehörige Bild nicht unnötig getrübt werden, sollten transparente und glaubwürdige Umweltbilanzen bereitgestellt, der Ausbau erneuerbarer Energiequellen und die Verbesserung der zur Herstellung von Akkumulatoren benötigten Rohstoffbeschaffung diskutiert werden. Daneben könnte eine stärkere öffentliche Kommunikation der Umweltvorteile das von der Gesellschaft wahrgenommene Image der Nutzer und damit auch deren Akzeptanz von eLM verbessern.

Bei der mangelnden Mehrzahlungsbereitschaft der Befragten in Bezug auf den Faktor Preis müssen äußere Faktoren mit in Betracht gezogen werden. So ist die Investitionsbereitschaft der Befragten aufgrund einer Reihe ökonomisch schwieriger Wirtschaftsjahre infolge unvorteilhafter Wettereinflüsse gedämpft und damit auch die Wirkung des Faktors Preis auf die Nutzerakzeptanz von eLM in diesem Zusammenhang negativ.

Der derzeit von den Experten als negativ wahrgenommene Einfluss des Faktors Politik auf die Akzeptanz von eLM könnte durch vertrauensschaffende Maßnahmen und die Präsentation plausibler Gesamtkonzepte, analog zur E-Mobilität in der Automobilbranche, möglicherweise ins Positive gedreht werden. Dabei können die von den Befragten bevorzugten finanziellen Förderinstrumente einen Schritt in diese Richtung darstellen, wobei ein Anstieg der Kaufpreise von eLM um ebenjenen Förderbetrag durch entsprechende Ausgestaltung der Maßnahmen vermieden werden sollte.

Für eine akzeptanzsteigernde Wirkung des Faktors Einstellung könnte die Weiterentwicklung elektrischer Anbaugeräte, die einen Beitrag zum „Precision Farming“ leisten, von Vorteil sein und einen Umstieg auf eLM attraktiver gestalten. 
Trotz der interessanten Ergebnisse hinsichtlich der Nutzerakzeptanz von eLM müssen, wie bei den meisten empirisch erhobenen Daten, bei der Interpretation der Ergebnisse eine Reihe von Einschränkungen beachtet werden. So handelt es sich bei den 15 befragten Landwirten größtenteils um konventionell wirtschaftende Ackerbauern aus dem Raum Niedersachsen, die im Gegensatz zu einigen viehhaltenden Betrieben bisher keine Erfahrungen mit der Nutzung von eLM sammeln konnten. Da Erfahrung mit technischen Innovationen nachweislich einen Einfluss auf deren Akzeptanz ausübt, könnten daher die Ergebnisse bspw. in der Innenwirtschaft anders ausfallen (MELENHORST und BOUWHUIS, 2004). Bei der kleinen, nicht repräsentativen Stichprobe handelt es sich bei der vorliegenden qualitativen Studie nur um eine explorative Untersuchung, die einer ersten Identifizierung und Einschätzung der Akzeptanz von eLM und deren Einflussfaktoren dient. Die Ergebnisse können dennoch als Hilfestellung für Landmaschinenhersteller und politische Entscheidungsträger genutzt werden, um die Verbreitung von eLM voranzutreiben, wobei die E-Mobilität in Konkurrenz zu verschiedenen anderen Antriebskonzepten, wie Wasserstoff, synthetische- oder Biokraftstoffe, steht. Diese explorative Studie bietet zudem Anknüpfungspunkte für weiterführende Forschung im Bereich der Akzeptanz von E-Mobilität in der Landwirtschaft. Es wäre bspw. denkbar, in methodischer Hinsicht ergänzende quantitative Forschungsarbeiten durchzuführen und unter inhaltlichen Aspekten auch das gesellschaftliche Meinungsbild einzufangen.

\section{Literatur}

AgRARHEUTE (2017): Maschinen unter Strom: So praxisreif sind Elektroantriebe. URL: https://www.ag rarheute.com/technik/maschinen-strom-so-praxisreif-elektroantriebe-533587 03.07.2020).

Aguilar-Gallegos, N., Munoz-Rodríguez, M., Santoyo-Cortés, H., Aguilar-Ávila, J. und L. KLERKX (2015): Information networks that generate economic value: A study on clusters of adopters of new or improved technologies and practices among oil palm growers in Mexico. In: Agricultural Systems 135 (2015): 122-132, doi: 10.1016/j.agsy.2015.01.003.

Altenburg, T., SChamp, E. W. und A. Chaudhary (2016): The emergence of electromobility: Comparing technological pathways in France, Germany, China and India. In: Science and Public Policy 43 (4): 464 475, doi: $10.1093 / \mathrm{scipol} / \mathrm{scv} 054$.

Aubert, B. A., Schroeder, A. und J. Grimaudo (2012): IT as enabler of sustainable farming: An empirical analysis of farmers' adoption decision of precision agriculture technology. In: Decision Support Systems 54 (1): 510-520, doi: 10.1016/j.dss.2012.07.002.

AUGENSTEIN, K. (2015): Analysing the potential for sustainable e-mobility - The case of Germany. In: Environmental Innovation and Societal Transitions 14: 101-115, doi: 10.1016/j.eist.2014.05.002.

Aumer, W., Lindner, M., GEIßLER, M. und T. HerlitZIUS (2008): Elektrischer Traktor: Vision oder Zukunft? In: Landtechnik 1/2008 (1): 14-15, doi: 10.15150/LT.2008.758.

Austin, E. J., Willock, J., Deary, I. J., Gibson, G. J., Dent, J. B., Edwards-Jones, G. und O. Morgan (1998): Empirical models of farmer behaviour using psychological, social and economic variables - Part I: Linear modelling. In: Agricultural Systems 58 (2): 203-224, doi: 10.1016/S0308-521X(98)00066-3. 
Auvinen, H., Järvi, T., Kloetzke, M., Kugler, U., Bühne, J.-A., Heinl, F., Kurte, J. und K. Esser (2016): Electromobility Scenarios: Research Findings to Inform Policy. In: Transportation Research Procedia 14: 2564-2573, doi: 10.1016/j.trpro.2016.05.346.

BMU (Bundesministerium für Umwelt, Naturschutz und nukleare Sicherheit) (2019): Der Klimaschutzplan 2050 - Die deutsche Klimaschutzstrategie. URL: https://www.bmu.de/themen/ klima-energie/klimaschutz/nationale-klimapolitik/klimaschutzplan-2050/(Abrufdatum: 18.08.2019).

BMVI (Bundesministerium für Verkehr und digitale Infrastruktur) (2019): Die Zukunft fährt elektrisch. URL: https://www.bmvi.de/DE/Themen/Mobilitaet/Elektromobilitaet/

Elektromobilitaet-kompakt/elektromobilitaet-kompakt.html (Abrufdatum: 03.06.2019).

BÖsche, K. V., Ester, P., Hock, T., RAhimzeI, E. und M. VOGEL (2017): Innovationen in der Elektromobilität. IKT für Elektromobilität III: Einbindung von gewerblichen Elektrofahrzeugen in Logistik-, Energie- und Mobilitätsinfrastrukturen. Bundesministerium für Wirtschaft und Energie, Berlin.

Bozem, K., NAgL, A., RATH, V. und A. Haubrock (2013): Elektromobilität: Kundensicht, Strategien, Geschäftsmodelle. Springer Fachmedien, Wiesbaden.

BRUnNENGRÄBER, A. und T. HAAS (2018): Vom Regen in die Traufe: die sozial-ökologischen Schattenseiten der E-Mobilität. In: GAIA - Ecological Perspectives for Science and Society 27 (3): 273-276, doi: 10.14512/gaia.27.3.4.

Bühne, J.-A., Gruschwitz, D., HÖlscher, J., KlÖtzKe, M., Kugler, U. und C. SchimecZEK (2015): How to promote electromobility for European car drivers? Obstacles to overcome for a broad market penetration. In: European Transport Research Review 7 (3), doi: 10.1007/s12544-015-0178-0.

Caetano, N. S., Mata, T. M., Martins, A. A. und M. C. Felgueiras (2017): New trends in energy production and utilization. In: Energy Procedia 107: 7-14, doi: 10.1016/j.egypro.2016.12.122.

Cansino, J., SÁnChez-BrazA, A. und T. SAnZ-DíAz (2018): Policy Instruments to Promote Electro-Mobility in the EU28: A Comprehensive Review. In: Sustainability 10 (7): 2507, 10.3390/su10072507.

Cavallo, E., Ferrari, E., Bollani, L. und M. Coccia (2014): Attitudes and behaviour of adopters of technological innovations in agricultural tractors: A case study in Italian agricultural system. In: Agricultural Systems 130 (2014): 44-54, doi: 10.1016/j.agsy.2014.05.012.

DütschKe, E., PAETZ, A.-G. und J. Wesche (2013): Integration Erneuerbarer Energien durch Elektromobilität - inwieweit sind Konsumenten bereit, einen Beitrag zu leisten? In: uwf UmweltWirtschaftsForum 21 (2013): 233-242, doi: 10.1007/s00550-013-0290-3.

EGBUE, O. und S. LONG (2012): Barriers to widespread adoption of electric vehicles: an analysis of consumer attitudes and perceptions. In: Energy Policy 48 (2012): 717-729, doi: 10.1016/j.enpol.2012.06.009.

EgBue, O., Long, S. und V. A. SAmaranayake (2017): Mass deployment of sustainable transportation: evaluation of factors that influence electric vehicle adoption. In: Clean Technologies and Environmental Policy 19 (2017): 1927-1939, doi: 10.1007/s10098-017-1375-4.

Erickson, B., Lowenberg-DeBoer, J. und J. BreAdFord (2017): 2017 Precision Agricultural Service Dealership Survey. URL: http://agribusiness.purdue.edu/files/file/croplife-purdue-2017-precision-dealersurvey-report.pdf (Abrufdatum: 21.09.2020).

FAR, S. T. und K. REZAEI-MOGHADDAM (2017): Determinants of Iranian agricultural consultants' intentions toward precision agriculture: Integrating innovativeness to the technology acceptance model. In: Journal of the Saudi Society of Agricultural Sciences 16 (3): 280-286, doi: 10.1016/j.jssas.2015.09.003.

FENDT (2020): Fendt hat die Zukunft fest im Visier. URL: https://www.fendt.com/de/11364.html. (Abrufdatum: 03.07.2020).

Franke, T., KREMS, J. und F. SCHMALFUß (2013): Adapting to the range of an electric vehicle - the relation of experience to subjectively available mobility resources. In: E. BRANDENBURG (Hrsg.): Grundlagen und Anwendungen der Mensch-Maschine-Interaktion. 10. Berliner Werkstatt Mensch-Maschine-Systeme, 10.-12. Oktober 2013, Berlin. Foundations and applications of human machine interaction, Universitätsverlag der TU, Berlin: 95-103. 
GLÄSER, J. und G. LAUDEL (2012): Experteninterviews und qualitative Inhaltsanalyse als Instrumente rekonstruierender Untersuchungen. Lehrbuch. VS - Verlag für Sozialwissenschaften, Wiesbaden.

GLOBISCH, J., SCHNEIDER, U. und E. DÜTSCHKE (2013): Acceptance of electric vehicles by commercial users in the electric mobility pilot regions in Germany. In: ECEEE Summer Study Proceedings: 973-983.

GNANN, T. und P. PlÖTZ (2011): Status Quo und Perspektiven der Elektromobilität in Deutschland. Fraunhofer ISI, Karlsruhe. URL: http://hdl.handle.net/10419/54753 (Abrufdatum: 22.10.2018).

gömann, H., Bender, A., Bolte, A., Dirksmeyer, W., Englert, H., Feil, J.-H., Frühauf, C., HauSChild, M., Krengel, S., Lilienthal, H. LÖPMEIER, F.-J., MÜller, J., Mußhoff, O., NATKhin, M., Offermann, F., Seidel, P., Schmidt, M., Seintsch, B., Steidl, J., Strohm, K. und Y. Zimmer (2015): Agrarrelevante Extremwetterlagen und Möglichkeiten von Risikomanagementsystemen: Studie im Auftrag des Bundesministeriums für Ernährung und Landwirtschaft (BMEL). URL: https://www.bmel.de/SharedDocs/Downloads/Landwirtschaft/Klima-und-Umwelt/Klima

schutz/AbschlussberichtProjektExtremwetterlagen.pdf?_blob=publicationFile_ (Abrufdatum: 03.06.2019).

GÖtZ, K., Sunderer, G., Birzle-Harder, B. und J. DefFner (2011): Attraktivität und Akzeptanz von Elektroautos. Arbeitspaket 1 des Projekts OPTUM: Optimierung der Umweltentlastungspotenziale von Elektrofahrzeugen, ISOE - Institut für sozialökologische Forschung, Frankfurt am Main.

GRÄBENER, S. (2017): Methodische Entwicklung und Bewertung von Elektrifizierungskonzepten für innerstädtische Nutzfahrzeuge. Dissertation. Fakultät V - Verkehrs- und Maschinensysteme, Technische Universität Berlin, Berlin.

Graham-Rowe, E., Gardner, B., Abraham, C., Skippon S., Dittmar, H., Hutchins, R. und J. STANNARD (2012): Mainstream consumers driving plug-in battery-electric and plug-in hybrid electric cars: A qualitative analysis of responses and evaluations. In: Transportation Research Part A: Policy and Practice 46 (1): 140-153, doi: 10.1016/j.tra.2011.09.008.

Hardman, S., ShiU, E. und R. STEINBERger-Wilckens (2016): Comparing high-end and low-end early adopters of battery electric vehicles. In: Transportation Research Part A: Policy and Practice 88: 40-57.

HERLITZIUS, T. (2018): Szenarien der Systemkonfiguration elektrifizierter Arbeitsmaschinen. In: KTBL (Hrsg.): In Zukunft elektrisch - Energiesysteme im ländlichen Raum. KTBL, Darmstadt: 75-85.

HEYMAnN, E. (2013): Evolution statt Revolution - die Zukunft der Elektromobilität. IW-Analysen, Nr. 84. IW Medien, Köln.

Holtz, G., XiA-BAuer, C., Roelfes, M., SChÜLe, R., VAlLentin, D. und L. MARTENS (2018): Competences of local and regional urban governance actors to support low-carbon transitions - development of a framework and its application to a case-study. In: Journal of Cleaner Production 177: 846-856, doi: 10.1016/j.jclepro.2017.12.137.

HÜsing, B., Bierhals, R., BÜhrlen, B., Friedewald, M., Kimpeler, S., Menrad, K., Wengel, J., ZimMER, R. und P. ZOCHE (2002): Technikakzeptanz und Nachfragemuster als Standortvorteil. Abschlussbericht. Fraunhofer-Institut für Systemtechnik und Innovationsforschung (Fraunhofer ISI), Karlsruhe.

JOHN DEERE (2020): Kabelgeführter mobiler und autonomer Maschinenverband - GridCON2 führt eine Vielzahl von Einzelentwicklungen zusammen. URL: http://www.gridcon-project.de/Beschreibung.html (Abrufdatum: 03.06.2019).

KAHN, M. E. (2007): Do greens drive Hummers or hybrids? Environmental ideology as a determinant of consumer choice. In: Journal of Environmental Economics and Management 54: 129-145.

Kaine, G., LeEs, J. und V. Wright (2007): An Approach to Predicting Demand for an Agricultural Innovation. In: Australasian Agribusiness Review 15 (7): 94-107.

KAIRIES, B. (2013): Marketing für Elektroautos. Akzeptanz als notwendige Bedingung für die Marktdurchdringung. Diplomica, Hamburg.

KEMFERT, C. (2018): Die Hitzewelle zeigt: Höchste Zeit für einen konsequenten Klimaschutz! In: DIW-Wochenbericht 85 (32): $689 \mathrm{ff}$. 
Kinm, A. und S. Trommer (2014): The new car market for electric vehicles and the potential for fuel substitution. In: Energy Policy 73: 147-157, doi: 10.1016/j.enpol.2014.05.021.

KollmanN, T. (1998): Akzeptanz innovativer Nutzungsgüter und -systeme: Konsequenzen für die Einführung von Telekommunikations- und Multimediasystemen. Reihe Neue betriebswirtschaftliche Forschung Band 239. Gabler Verlag, Wiesbaden.

KRAMER (2020): KL25.5e - Der Kramer KL25.5e - eine Fahrzeugklasse für sich. URL: https:/ /www.krameronline.com/de/branchen-produkte/landwirtschaft/radlader/radlader-4t/model/kl255e/ (Abrufdatum: 03.07.2020).

Krupa, J. S., Rizzo, D. M., Eppstein, M. J., Brad Lanute, D., GaAlema, D. E., Lakkaraju, K. und C. E. WARRENDER (2014): Analysis of a consumer survey on plug-in hybrid electric vehicles. In: Transportation Research Part A: Policy Practice 64: 14-31, doi: 10.1016/j.tra.2014.02.019.

LANE, B. und S. POTTER (2007): The adoption of cleaner vehicles in the UK: exploring the consumer attitudeaction gap. In: Journal of Cleaner Production 15 (11-12): 1085-1092, doi: 10.1016/j.jclepro.2006.05.026.

LONG, T. B., BLOK, V. und I. CONINX (2016): Barriers to the adoption and diffusion of technological innovations for climate-smart agriculture in Europe: evidence from the Netherlands, France, Switzerland and Italy. In: Journal of Cleaner Production 112 (1): 9-21, doi: 10.1016/j.jclepro.2015.06.044.

MAYRING, P. (2015): Qualitative Inhaltsanalyse. Grundlagen und Techniken. Beltz Pädagogik, Weinheim.

Melenhorst, A.-S. und D. G. Bouwhuis (2004): When do older adults consider the internet? An exploratory study of benefit perception. In: Gerontechnology 3 (2): 89-101, doi: 10.4017/gt.2004.03.02.004.00.

MiSERIOR (2018): Rohstoffe für die Energiewende. Bischöfliches Hilfswerk MISEREOR e.V., Aachen. URL: https://www.misereor.de/fileadmin/publikationen/studie-rohstoffe-fuer-die-energiewende.pdf (Abrufdatum: 12.2.2019).

MoOns., I. und P. DE Pelsmacker (2012): Emotions as determinants of electric car usage intention. In: Journal of Marketing Management 28 (3-4): 195-237, doi: 10.1080/0267257X.2012.659007.

NPE (2018): Fortschrittsbericht 2018 - Markthochlaufphase. URL: http://nationale-plattform-elektromobi litaet.de/fileadmin/user_upload/Redaktion/NPE_Fortschrittsbericht_2018_barrierefrei.pdf. (Abrufdatum: 10.4.2019).

PATTON, M. Q. (2015): Qualitative research \& evaluation methods: integrating theory and practise. SAGE, Los Angeles.

Pierpaoli, E., Carli, G., Pignatti, E. und M. Canavari (2013): Drivers of Precision Agriculture Technologies Adoption: A Literature Review. In: Procedia Technology 8: 61-69, doi: 10.1016/j.protcy.2013.11.010.

PETERS, A. und E. DÜTSCHKE (2010): Zur Nutzerakzeptanz von Elektromobilität. Analyse aus Expertensicht. Fraunhofer Institut, Karlsruhe.

Peters, A. und J. HoffmanN (2011): Nutzerakzeptanz von Elektromobilität. Eine empirische Studie zu attraktiven Nutzungsvarianten, Fahrzeugkonzepten und Geschäftsmodellen aus Sicht potenzieller Nutzer. Fraunhofer Institut, Karlsruhe.

Peters, A., Doll, C., PlÖtz, P., Thielmann, A., SAuer, A., ZAnker, C., Wietschel, M. und W. Schade (2013): Konzepte der Elektromobilität. Ihre Bedeutung für Wirtschaft, Gesellschaft und Umwelt. Nomos Verlagsgesellschaft mbH \& Co. KG, Baden-Baden.

PICKEL, P. (2018): Elektrifizierung in der Landwirtschaft - wo und wofür? In: KTBL (Hrsg.): In Zukunft elektrisch - Energiesysteme im ländlichen Raum. KTBL, Darmstadt: 56-74.

PICKEL, P. (2019a): Electricity for tractors and tractor-implement systems. Club of Bologna, 29th Members Meeting, Hannover 10.-11.11.2019. URL: https://www.clubofbologna.org/ew/ew _proceedings/2019_KNR_S2.1_Pickel_original.pdf (Abrufdatum: 03.07.2020). 
PICKEL, P. (2019b): Es ist schwierig den Strom auf die Maschine zu bringen. Interview vom 31.10.2019. URL: https://www.deere.de/de/blog/articles/technik/elektrifizierung-landwirtschaft-john-deere-traktor/ (Abrufdatum: 21.09.2020).

QuiRING, O. (2006): Methodische Aspekte der Akzeptanzforschung bei interaktiven Medientechnologien. In: Münchener Beiträge zur Kommunikationswissenschaft 6 (2006): 1-29, doi: 10.5282/ubm/epub.1348.

RENN, O. (2005): Technikakzeptanz: Lehren und Rückschlüsse der Akzeptanzforschung für die Bewältigung des technischen Wandels. In: Technikfolgenabschätzung - Theorie und Praxis 3 (14): 29-37, doi: 10.14512/tatup.14.3.29.

REZVANI, Z., JANSSON, J. und J. BODIN (2015): Advances in consumer electric vehicle adoption research: a review and research agenda. In: Transportation Research Part D: Transport Environment 34 (2015): 122-136, doi: 10.1016/j.trd.2014.10.010.

RIEDEMANN, P. (2011): Kundenakzeptanz von Innovationen im Produktentwicklungsprozess. Dissertation. Freie Universität Berlin, Berlin.

Riedner, L., MAir, C., ZimeK, M., BrudermanN, T. und T. STERn (2019): E-mobility in agriculture: differences in perception between experienced and non-experienced electric vehicle users. In: Clean Technology and Environmental Policy (2019) 21: 55-67, doi: 10.1007/s10098-018-1615-2.

SCHÄFER, M. und D. KePPLER (2013): Modelle der technikorientierten Akzeptanzforschung. Überblick und Reflexion am Beispiel eines Forschungsprojekts zur Implementierung innovativer EnergieeffizienzMaßnahmen. In: TU Berlin discussion paper 34 (2013), doi: 10.14279/depositonce-4461.

SChimmelpfennig, D. und R. Ebel (2016): Sequential Adoption and Cost Savings from Precision Agriculture. In: Journal of Agricultural and Resource Economics 41 (1): 97-115.

Schneider, U., DÜtschKe, E. und A. Peters (2014): How Does the Actual Usage of Electric Vehicles Influence Consumer Acceptance? In: HÜlsmanN, M. und D. FORNAHL (Hrsg.): Evolutionary Paths towards the Mobility Patterns of the Future. Springer, Berlin/Heidelberg.

SKIPPON, S. und M. GARWOOD (2011): Responses to battery electric vehicles: UK consumer attitudes and attributions of symbolic meaning following direct experience to reduce psychological distance. In: Transportation Research Part D: Transport and Environment 16 (7): 525-531, doi: 10.1016/j.trd.2011.05.005.

SILOKING (2020): Siloking e.0 - Produktprospekt. URL: https://www.siloking.com/de/siloking-produkt uebersicht/futtermischwagen-truckline\#downloads (Abrufdatum: 03.07.2020).

STOEHR, M., GiGLMAIER, S. und R. BERLET (2015): Folgenabschätzung zum Einsatz batteriebetriebener vollelektrifizierter Landmaschinen. B.A.U.M. Consult GmbH. URL: https://www.baumgroup. de/fileadmin/interface/files/HDSAVATEVA-142016151526-CJTYIRXHHM.pdf (Abrufdatum: 03.07.2020).

STÖHR, M. und B. HACKENBERG (2018): Enhancing Synergy Effects Between The Electrification Of Agricultural Machines And Renewable Energy Deployment With Semi-Stationary Energy Storage In Rural Grids. In: Energy Procedia 155: 179-187, doi: 10.1016/j.egypro.2018.11.057.

Thompson, N. M., Bir, C., Widmar, D. A. und J. R. MinterT (2018): Farmer Perceptions of Precision Agriculture Technology Benefits. In: Journal of Agricultural and Applied Economics 51 (1): 142-163, doi: $10.1017 /$ aae.2018.27.

VON JEINSEN, T., HePpe, H. und L. THEUvSEN (2018): Determinanten der Akzeptanz technischer Innovationen in der Landwirtschaft. In: Ruckelshausen, A., MeYer-Aurich, A., BOrCHARD, K., Hofacker, C., LOY, J.-P., SCHWERDTFEgER, R., SundermeIER, H.-H., Floto, H. und B. Theuvsen (Hrsg.): Digitale Marktplätze und Plattformen, Lecture Notes in Informatics (LNI). Gesellschaft für Informatik, Bonn: 127-130.

Voss, J., SCHAPER, C., SPILLER, A. und L. TheuvSEN (2008): Innovationsverhalten in der deutschen Landwirtschaft - Empirische Ergebnisse am Beispiel der Biogasnutzung. In: BERG, E., HARTMANN, M., HECKELEI, T., HOLM-MÜLLER, K. und G. SCHIEFER (Hrsg,): Risiken in der Agrar- und Ernährungswirtschaft und ihre Bewältigung, Schriften der GEWISOLA 44. GEWISLOA, Münster: 379-391. 
WEIDEMANN (2020): Weidemann 1160 eHoftrac. URL: http://www.weidemann.de/de/hoftrac/model/ 1160-ehoftrac/ (Abrufdatum: 03.07.2020).

YAY, M. (2012): Elektromobilität. Theoretische Grundlagen, Herausforderungen sowie Chancen und Risiken der Elektromobilitaet, diskutiert an den Umsetzungsmöglichkeiten in die Praxis. Lang Peter GmbH Internationaler Verlag der Wissenschaften, Frankfurt. 


\section{Anhang}

Tabelle A1: Demografische und betriebliche Daten der Interviewpartner

\begin{tabular}{|c|c|c|c|c|c|c|c|}
\hline $\begin{array}{l}\text { Lfd. } \\
\text { Nr. }\end{array}$ & $\begin{array}{c}\text { Ge- } \\
\text { schlecht }\end{array}$ & Alter & Ausbildung & Landkreis & $\begin{array}{c}\text { Erwerbs- } \\
\text { form }\end{array}$ & Betriebszweig & $\begin{array}{c}\text { Fläche } \\
\text { in ha }\end{array}$ \\
\hline 1 & männlich & 24 & Landwirt & Hildesheim & Haupterwerb & $\begin{array}{l}\text { Direktvermark- } \\
\text { tung }\end{array}$ & 30 \\
\hline 2 & männlich & 43 & Studium & Hildesheim & Haupterwerb & Ackerbau & 190 \\
\hline 3 & männlich & 55 & Meister & Hildesheim & Haupterwerb & $\begin{array}{c}\text { Ackerbau und } \\
\text { Schweinehaltung }\end{array}$ & 200 \\
\hline 4 & männlich & 58 & keine & Hildesheim & Haupterwerb & Ackerbau & 85 \\
\hline 5 & männlich & 35 & $\begin{array}{l}\text { Agrarbetriebs- } \\
\text { wirt }\end{array}$ & Hannover & Haupterwerb & $\begin{array}{l}\text { Ackerbau und } \\
\text { Milchvieh }\end{array}$ & 175 \\
\hline 6 & männlich & 50 & Studium & Hildesheim & Haupterwerb & Ackerbau & 140 \\
\hline 7 & männlich & 28 & Studium & $\begin{array}{l}\text { Vor-pom- } \\
\text { mern- } \\
\text { Greifswald }\end{array}$ & Haupterwerb & Ackerbau & 1000 \\
\hline 8 & männlich & 29 & Studium & Hildesheim & $\begin{array}{l}\text { Nebener- } \\
\text { werb }\end{array}$ & Ackerbau & 175 \\
\hline 9 & männlich & 64 & Studium & Hildesheim & Haupterwerb & Ackerbau & 400 \\
\hline 10 & männlich & 29 & $\begin{array}{c}\text { Agrarbetriebs- } \\
\text { wirt }\end{array}$ & Hannover & Haupterwerb & $\begin{array}{l}\text { Ackerbau und } \\
\text { Milchvieh }\end{array}$ & 135 \\
\hline 11 & männlich & 40 & $\begin{array}{c}\text { Agrarbetriebs- } \\
\text { wirt }\end{array}$ & Northeim & Haupterwerb & Ackerbau & 95 \\
\hline 12 & männlich & 35 & $\begin{array}{c}\text { Agrarbetriebs- } \\
\text { wirt }\end{array}$ & Hannover & $\begin{array}{l}\text { Nebener- } \\
\text { werb }\end{array}$ & Ackerbau & 44 \\
\hline 13 & männlich & 36 & Meister & Heidekreis & Haupterwerb & Ackerbau & 260 \\
\hline 14 & männlich & 52 & $\begin{array}{c}\text { Agrarbetriebs- } \\
\text { wirt }\end{array}$ & Hildesheim & Haupterwerb & Ackerbau & 525 \\
\hline 15 & männlich & 43 & $\begin{array}{c}\text { Agrarbetriebs- } \\
\text { wirt }\end{array}$ & $\begin{array}{l}\text { Holzmin- } \\
\text { den }\end{array}$ & $\begin{array}{l}\text { Nebener- } \\
\text { werb }\end{array}$ & $\begin{array}{c}\text { Ackerbau und } \\
\text { Milchvieh }\end{array}$ & 137 \\
\hline$\overline{\mathrm{X}}$ & & & & & & & 239,4 \\
\hline
\end{tabular}


Tabelle A2: Ergebnisse für den Faktor Offenheit

\begin{tabular}{lll}
\hline Merkmalsausprägung & \multicolumn{1}{c}{ Ergebnisse der Befragung } \\
\hline Aufgeschlossene Gruppe & - & Offenheit wichtig, um Verbesserungen für den Betrieb wahrzunehmen \\
& - & höhere Offenheit wird als wichtig für Wahrnehmung von Informatio- \\
& nen gewertet \\
& - & Wahrnehmung der Informationen als Voraussetzung für wichtige be- \\
& triebliche Entscheidungen \\
Abwartende Gruppe & - & Wahrnehmung von Informationen wird nicht aktiv betrieben \\
& - & eLM kein Schwerpunkt \\
Weitere Einflüsse auf die & - & Abwarten der technischen Entwicklung und spätere Adoption \\
Offenheit & - & andere berufliche Tätigkeit \\
Kosten-Nutzen-Relation & - & Alter \\
& - & Ausbildungsgrad \\
& fenheit für eLM \\
& - & Offenheit abhängig von betrieblichen Strukturen/Einsatzbereichen \\
& und Finanzierungsmöglichkeiten \\
& - & über die Finanzierungsmöglichkeiten hat Preis der Technik Einfluss \\
& auf die Offenheit \\
& - & ausprobieren eLM steigert wahrgenommenen Nutzen und führt zu hö- \\
& herer Offenheit
\end{tabular}

Quelle: Eigene Darstellung

Tabelle A3: Ergebnisse für den Faktor Informationen

\begin{tabular}{lll}
\hline Merkmalsausprägung & \multicolumn{1}{c}{ Ergebnisse der Befragung } \\
\hline Kontakt mit & - & wenig Informationen vorhanden aus Sicht der Landwirte und wird ne- \\
Informationen & gativ bewertet \\
& - & unterschiedliche Wahrnehmung der Medien bezüglich Informationen \\
& - & Messen erfordern eigenes Interesse zur Informationssuche \\
& - & Fachzeitschriften: Informationen werden dargeboten, keine aktive Su- \\
& che notwendig \\
Informationsstand & - & Faktor Offenheit beeinflusst Wahrnehmung von Informationen \\
& - & abhängig von wahrgenommenem Nutzen und betrieblichen Einsatzbe- \\
& reichen \\
& - & Faktor Offenheit für Aufbau eines Wissensstandes wichtig \\
& - & bisher keine umfassenden Informationen angeboten bekommen, son- \\
& dern nur Wissen über einzelne Aspekte von eLM vorhanden \\
Wichtige Informationen & besserer Informationsstand über konventionelle Antriebe \\
& - & Handlungsaufforderung das Gesamtkonzept von eLM besser darzule- \\
& gen \\
- & Vergleichstests zu konventionellen Landmaschinen \\
- & Einschätzungen von Experten aus der Technikbranche \\
- & praxisbezogene Informationen \\
- & Aufzeigen eines Kosten-Nutzen-Verhältnisses, vor allem technische \\
& Aspekte/Vorteile und Kaufpreise, wichtig für eine subjektive Ein- \\
& schätzung \\
- & eigenes Ausprobieren wichtige Quelle von Informationen, teilweise \\
& Voraussetzung für den Kauf einer Landmaschine \\
\hline
\end{tabular}

Quelle: Eigene Darstellung 
Tabelle A4: Ergebnisse für den Faktor Technik

\begin{tabular}{|c|c|}
\hline Merkmalsausprägung & Ergebnisse der Befragung \\
\hline Vorteile & $\begin{array}{l}\text { - Leistung, bessere Kraftübertragung, weniger Energieverluste, Energie- } \\
\text { effizienz; auch im Vergleich zu konventionellen Landmaschinen } \\
\text { - } \\
\text { geringere Wartung, einhergehend mit geringerem Verschleiß der Ma- } \\
\text { schinen und weniger Arbeitsaufwand } \\
\text { - } \quad \text { einfacherer technischer Aufbau, vor allem Wegfall des Getriebes und } \\
\text { Ersatz von Öl- und Hydraulikbauteilen } \\
\text { - } \quad \text { Einsparungen durch Nutzung von Strom gegenüber Diesel } \\
\text { - Nutzung elektrifizierter Anbaugeräte, bessere Steuer- und Dosierbar- } \\
\text { keit, Optimierung von Aufwandmengen bei Dünger und Pflanzen- } \\
\text { schutzmitteln }\end{array}$ \\
\hline Nachteile & $\begin{array}{l}\text { - allgemeine technische Reife; mitunter Vergleich zu elektrischen Auto- } \\
\text { mobilen } \\
\text { - Kontakt zu Werkstätten und Reparaturen, Personenschutz beim Arbei- } \\
\text { ten mit einem Hochspannungsbordnetz an eLM } \\
\text { - Batteriespeicher bezüglich Reichweite und Energiedichte als unzu- } \\
\text { reichend wahrgenommen, geforderte Einsatzzeit zwischen } 8 \text { und } 14 \\
\text { Stunden }\end{array}$ \\
\hline Anwendung in der & - breites Einsatzspektrum und ähnliche Flexibilität wie herkömmlich \\
\hline & Landmaschinen gefordert \\
\hline Einsatzbereiche & $\begin{array}{l}\text { - Ackerbau weniger als Einsatzbereich wahrgenommen; Potenzial wird } \\
\text { aber beim Einsatz für Pflegemaßnahmen gesehen } \\
\text { - Viehhaltende Betrieb erscheinen den Befragten geeigneter aufgrund } \\
\text { des Entwicklungsstandes eLM } \\
\text { - vor allem Bereiche mit täglicher Routine und kurzen Einsatzzeiträumen } \\
\text { - Emissionsfreier Betrieb in Ställen und Gebäuden positiv wahrgenom- } \\
\text { men }\end{array}$ \\
\hline $\begin{array}{l}\text { Ladeinfrastruktur und } \\
\text { Ladezyklen }\end{array}$ & $\begin{array}{l}\text { - Umsetzung einer Ladeinfrastruktur an der Hofstelle mehrheitlich un- } \\
\text { problematisch wahrgenommen, teilweise aber Unsicherheit über Kos- } \\
\text { tenaufwand und Leistung der verfügbaren Stromnetze } \\
\text { - kritische Sicht auf das Ladungsmanagement in Arbeitsspitzen, unter } \\
\text { Zeitdruck oder bei Wettereinflüssen } \\
\text { - Übernacht-Laden als Präferenz } \\
\text { - Verhältnis von Einsatz- und Aufladezeit bei aktuellem technischem } \\
\text { Stand kritisch wahrgenommen, kürzere Aufladezeiten gefordert }\end{array}$ \\
\hline Weitere Einflüsse & $\begin{array}{l}\text { - Verfügbarkeit am Markt beeinflusst Wahrnehmung der technischen } \\
\text { Reife und bietet Möglichkeit eLM zu testen }\end{array}$ \\
\hline
\end{tabular}

Quelle: Eigene Darstellung 
Tabelle A5: Ergebnisse für den Faktor Umwelt

\begin{tabular}{|c|c|}
\hline Merkmalsausprägung & Ergebnisse der Befragung \\
\hline Vorteile & $\begin{array}{l}\text { - } \quad \text { emissionsfreier Betrieb der Maschinen } \\
\text { - } \quad \text { Lärmreduktion, gerade in Nähe zu bewohnten Gebieten } \\
\text { - } \quad \text { Unabhängigkeit von Diesel, Kostenreduktion und Ressourcenscho- } \\
\text { nung durch Nutzung von Strom } \\
\text { - } \quad \text { Nutzung eigen erzeugten Stromes aus erneuerbaren Energien inkl. } \\
\text { Kostenvorteilen und attraktiv bei zukünftigem Wegfall von Einspeise- } \\
\text { vergütungen } \\
\text { - } \\
\text { aber Vorteile kritisch betrachtet, besonders aufgrund der Herstellung, } \\
\text { Unklarheiten über Umweltbilanzen und Weiterentwicklungen bei kon- } \\
\text { ventioneller Technik }\end{array}$ \\
\hline Nachteile & $\begin{array}{l}\text { - unklare Umweltbilanz von eLM, besonders Produktion und Herstel- } \\
\text { lung der Batteriespeicher kritisch betrachtet } \\
\text { - } \quad \text { überregionale Emissionen, wenn Betrieb der Maschinen nur mit kon- } \\
\text { ventionellem Strom möglich ist } \\
\text { - } \quad \text { regenerative Energien ebenfalls nicht unkritisch gesehen, z.B. Herstel- } \\
\text { lung von Photovoltaikanlagen und Energiebilanz von Biogasanlagen }\end{array}$ \\
\hline $\begin{array}{l}\text { Image der } \\
\text { Landwirtschaft }\end{array}$ & 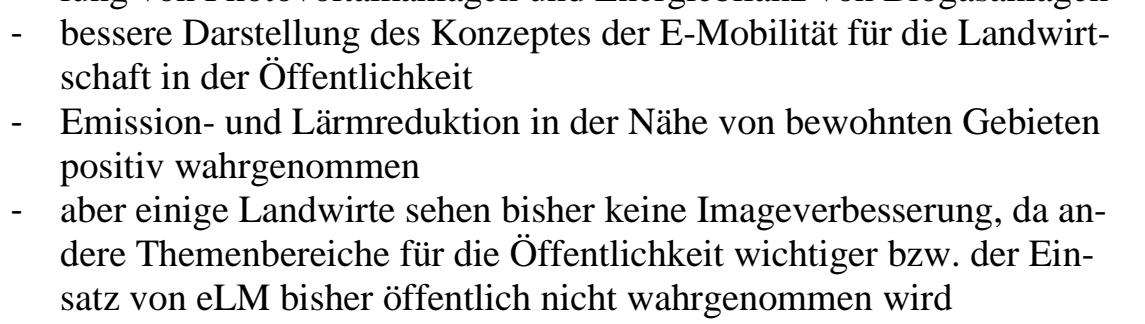 \\
\hline $\begin{array}{l}\text { Nutzung zugunsten der } \\
\text { Umweltvorteile }\end{array}$ & $\begin{array}{l}\text { - einige Landwirte bereit für Umweltvorteile in einem gewissen Rah- } \\
\text { men höhere Kaufpreise zu akzeptieren } \\
\text { - mehrheitlich Preis bzw. Wirtschaftlichkeit wichtiger } \\
\text { - Viehbetriebe: technische Aspekte für die betrieblichen Einsatzberei- } \\
\text { che im Vordergrund }\end{array}$ \\
\hline
\end{tabular}

Quelle: Eigene Darstellung

Tabelle A6: Ergebnisse für den Faktor Preis

\begin{tabular}{|c|c|}
\hline Merkmalsausprägung & Ergebnisse der Befragung \\
\hline $\begin{array}{l}\text { Akzeptanz höherer } \\
\text { Kaufpreise }\end{array}$ & $\begin{array}{l}\text { - nur von wenigen Landwirten oder unter bestimmten Umständen ak- } \\
\text { zeptierbar, Preis ist aber nicht alleiniges Bewertungskriterium } \\
\text { - Bewertung anhand der Wirtschaftlichkeit der Maschine und im Ver- } \\
\text { gleich zu konventionellen Landmaschinen } \\
\text { - genannt werden Betriebsgröße, Liquidität des Betriebes, Unterhalts- } \\
\text { kosten } \\
\text { - } \\
\text { technische Leistung der Maschine ebenfalls Ursache für Bewertung } \\
\text { des Kaufpreises } \\
\text { - allgemeine Konkurrenz am Markt zu konventionellen Landmaschinen } \\
\text { beeinflusst Bewertung des Preises } \\
\text { - äußere Einflüsse: fehlende Einnahmen, angespannte Marktsituationen } \\
\text { wirken negativ auf Akzeptanz höherer Preise }\end{array}$ \\
\hline
\end{tabular}

Quelle: Eigene Darstellung 
Tabelle A7: Ergebnisse für den Faktor Politik

\begin{tabular}{lll}
\hline Merkmalsausprägung & \multicolumn{1}{c}{ Ergebnisse der Befragung } \\
\hline Aktuelle Wahrnehmung & - & eLM kein Schwerpunkt in der Politik \\
der Politik & - & grundsätzlich aber wichtige Rolle für Verbreitung eLM \\
& - & Politik wichtig für betriebliche Planungssicherheit im Hinblick auf In- \\
& vestitionen in eLM \\
Politische Instrumente & - & Darstellung eines Gesamtkonzeptes für die E-Mobilität in der Land- \\
& wirtschaft mit Ausbau der Infrastruktur zur Sicherung des Strombe- \\
& darfes \\
- & Senkung bei Kfz-Steuern aufgrund der Steuerbefreiung landwirt- \\
& schaftlichen Maschinen unattraktiv \\
& - & andere steuerliche Bewertung in Finanzierungssystem positiv wahrge- \\
& nommen \\
- & Förderungen beim Maschinenkauf besonders positiv aufgefasst, da \\
& wirtschaftlicher Vorteil entsteht, Ausgleich der höheren Kaufpreis \\
& eLM \\
- & bei Förderungen aber auch negative Erfahrungen berichtet und teil- \\
& weise kritisch gesehen \\
- & Förderung der Forschung im Bereich eLM positiv wahrgenommen \\
\hline
\end{tabular}

Quelle: Eigene Darstellung

Tabelle A8: Ergebnisse für den Faktor Einstellung

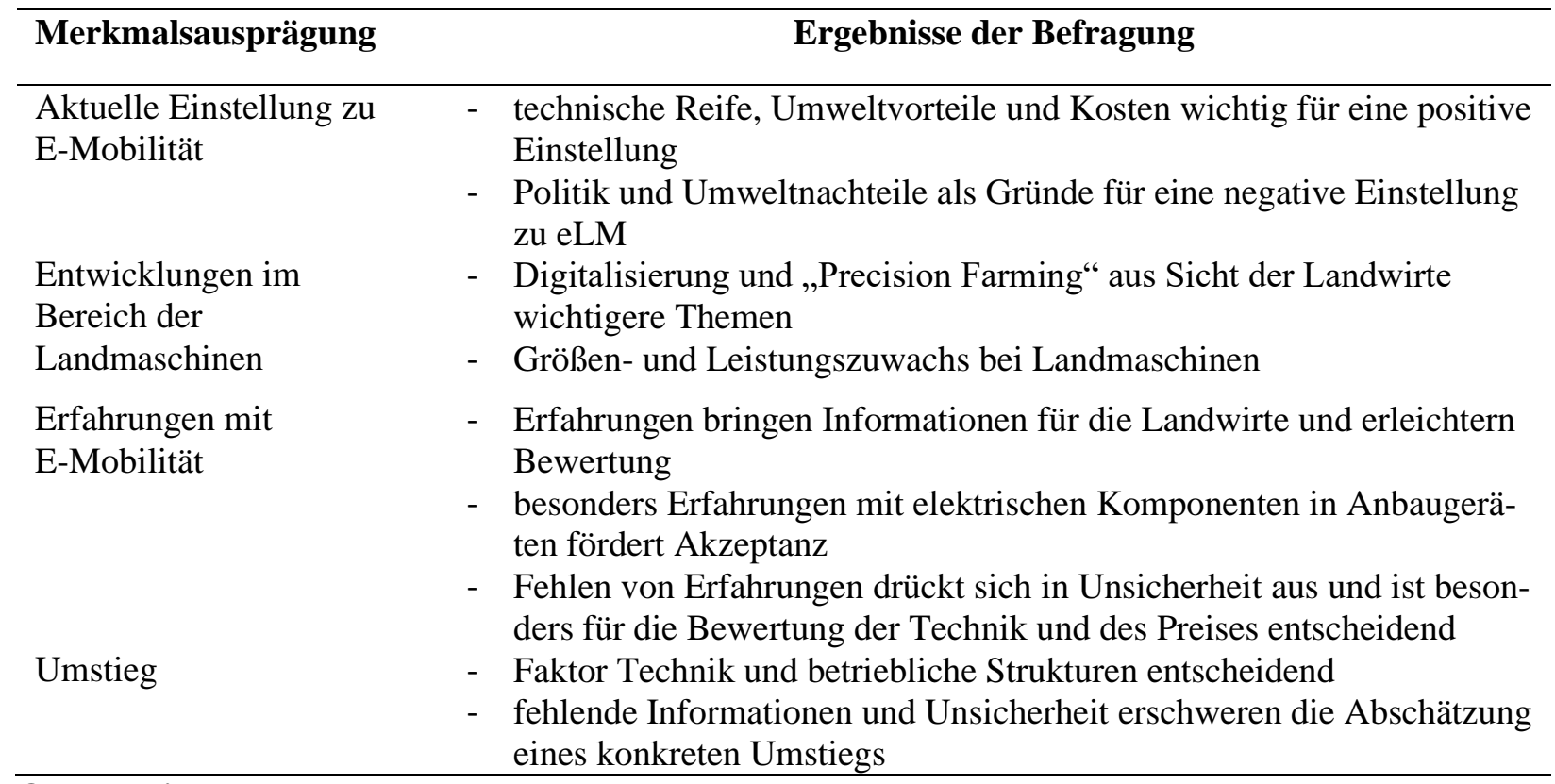

Quelle: Eigene Darstellung 


\section{Interviewleitfaden}

\section{Einstellung}

1. Beschreiben Sie bitte, wie in Zukunft der Einsatz von Landmaschinen Ihrer Meinung nach aussehen könnte.

a. Ggf. Nachfrage: Wo sehen Sie da die Elektromobilität/elektrische Landmaschinen?

2. Wie ist ihre persönliche Einstellung zum Thema der elektrischen Landmaschinen?

3. Wie stellen Sie sich in 5 oder 10 Jahren eine attraktive Nutzung oder Nutzungsmöglichkeiten von elektrischen Landmaschinen vor?

4. Welche Erfahrungen haben Sie schon mit elektrischen Landmaschinen gemacht?

\section{Treiber und Hemmnisse}

5. Welche Faktoren begünstigen die Verbreitung elektrischer Landmaschinen? Welche Vorteile sehen Sie bei elektrischen Landmaschinen?

6. Welche Faktoren stehen der Verbreitung elektrischer Landmaschinen im Weg? Welche Nachteile sehen Sie bei elektrischen Landmaschinen?

7. Was muss sich ändern, damit elektrische Landmaschinen für Sie als Kunde/Nutzer attraktiv werden?

\section{Offenheit für Innovation}

8. Wie schätzen Sie generell Ihre Offenheit gegenüber neuen Innovationen ein?

9. Wie innovativ schätzen Sie Ihren Betrieb anhand der Skala ein?

\begin{tabular}{ccccc}
\hline $\begin{array}{c}1 \\
\begin{array}{c}\text { Gar nicht } \\
\text { innovativ }\end{array}\end{array}$ & $\begin{array}{c}\text { Eher nicht } \\
\text { innovativ }\end{array}$ & Teils/teils & Eher innovativ & Sehr innovativ \\
\hline$\square$ & $\square$ & $\square$ & $\square$ & $\square$ \\
\hline
\end{tabular}

10. Wie innovativ schätzen Sie sich im Vergleich zu Ihren Kollegen ein?

\begin{tabular}{ccccc}
\hline $\begin{array}{c}1 \\
\begin{array}{c}\text { Gar nicht } \\
\text { innovativ }\end{array}\end{array}$ & $\begin{array}{c}\text { Eher nicht } \\
\text { innovativ }\end{array}$ & Teils/teils & Eher innovativ & Sehr innovativ \\
\hline$\square$ & $\square$ & $\square$ & $\square$ & $\square$ \\
\hline
\end{tabular}

11. Wie schätzen Sie Ihr Interesse am Thema elektrischer Landmaschinen ein, wenn es um Innovationen geht?

\section{Information}

12. Kommen Sie häufig in Kontakt mit Informationen zu elektrischen Landmaschinen, z.B. in landwirtschaftlichen Zeitschriften oder auf Messen?

13. Fühlen Sie sich ihrer Meinung nach gut informiert über elektrische Landmaschinen?

a. Ggf. Nachfrage: Wie empfinden Sie das im Vergleich zu dieselbetriebenen Landmaschinen?

14. Welche Informationen bezüglich der elektrischen Landmaschinen sind für Sie wichtig, um eine Einschätzung vorzunehmen? 
a. Ggf. Nachfrage: Würden Vorführungen oder eigenes Ausprobieren dabei helfen?

\section{Technik}

15. Welche Vorteile von elektrischen Landmaschinen sehen Sie speziell aus technischer Sicht?

a. Wie bewerten Sie die genauere Steuer und Regelbarkeit?

b. Wie bewerten Sie den höheren Wirkungsgrad des Antriebstranges $>90 \%$ ?

c. Wie bewerten Sie die geringere Wartung?

16. Wo sehen Sie hinsichtlich der technischen Ausreifung Verbesserungsbedarf bei elektrischen Landmaschinen?

a. Batterieleistung?

17. Halten Sie die Technik grundsätzlich für geeignet, um Anwendung in der Landwirtschaft zu finden?

a. Wo sehen Sie hauptsächlich Einsatzbereiche?

18. Aktuelle elektrische Landmaschinen haben eine Einsatzzeit und Ladezeit von ca.5 Std. Wie bewerten Sie das für Ihren Betriebsablauf?

a. Würden Sie ihren Betriebsablauf an technische Gegebenheiten anpassen?

19. Empfinden Sie das Aufladen der elektrischen Landmaschinen auf Ihrem Betrieb ohne größeren Aufwand für machbar?

a. Ggf. Nachfrage: Was sind Probleme, die sich für Sie ergeben?

\section{Umwelt}

20. Wie bewerten Sie das Thema Umweltschutz und Nachhaltigkeit für Sie und Ihren Betrieb?

21. Welche Vorteile sehen Sie speziell in Bezug auf die Umwelt beim Einsatz von elektrischen Landmaschinen?

a. Wie bewerten Sie die Reduktion/Unabhängigkeit von Diesel?

b. Wie bewerten Sie die Lärmreduktion?

c. Wie bewerten Sie den emissionsfreien Betrieb der Maschinen?

22. Welche Nachteile für die Umwelt sehen sie durch den Einsatz von elektrischen Landmaschinen?

a. Wie bewerten Sie es, wenn der Strom zum Laden nicht aus erneuerbaren Energien kommt?

b. Wie bewerten Sie den Rohstoffbedarf zur Herstellung der elektrischen Maschinen?

23. Wie bewerten Sie den Einsatz elektrischer Landmaschinen in Bezug auf das Image der Landwirtschaft, z.B. bei Umweltverbänden oder in der Bevölkerung?

24. Elektrische Maschinen ermöglichen auch eigen produzierten Strom zum Aufladen zu verwenden. Wie schätzen Sie dies für Ihre Akzeptanz der elektrischen Landmaschinen ein?

25. Gibt es Nachteile der elektrischen Landmaschinen, die sie zugunsten der Umweltvorteile in Kauf nehmen würden?

a. Mehrkosten?

b. Technische Reife der Landmaschinen?

\section{Preis}

26. Elektrofahrzeuge sind in der Regel teurer als vergleichbare Fahrzeuge mit Dieselmotor. Wie schätzen Sie das in Bezug auf die Wirtschaftlichkeit Ihres landwirtschaftlichen Betriebes ein?

27. Wären Sie bereit für einen geringeren $\mathrm{CO}_{2}$-Ausstoß höhere Kaufpreise zu akzeptieren? 
a. Gibt es technische Vorteile für den Sie höhere Kaufpreise akzeptieren?

\section{Politik}

28. Welche Rolle spielt für Sie die Politik bei der Verbreitung der elektrischen Landmaschinen?

29. Was sollte die Politik ihrer Meinung nach unterstützen, um elektrische Landmaschinen für die Landwirte attraktiver zu machen?

a. Wie würden Sie steuerliche Vorteile, z.B. bei Kfz-Steuern, für Ihren Betrieb bewerten?

b. Wie würden Sie Prämien oder Subventionen beim Kauf von elektrischen Maschinen für Ihren Betrieb bewerten?

\section{Nachbereitung}

30. Welche der angesprochenen Faktoren sind Ihnen am wichtigsten?

Was müsste noch erfüllt werden, damit ein Umstieg in Betracht käme? 


\section{I.2 Akzeptanz autonomer Feldroboter im Ackerbaueinsatz - Status quo und Forschungsbedarf}

Friedrich Rübcke von Veltheim, Ludwig Theuvsen und Heinke Heise

Geleisteter Eigenanteil an dem Beitrag

\begin{tabular}{|c|c|c|c|c|c|}
\hline $\begin{array}{l}\text { Literatur- } \\
\text { recherche }\end{array}$ & $\begin{array}{l}\text { Entwicklung } \\
\text { des } \\
\text { Forschungs- } \\
\text { rahmens }\end{array}$ & $\begin{array}{l}\text { Konzept und } \\
\text { Design der Stu- } \\
\text { die }\end{array}$ & $\begin{array}{l}\text { Auswahl, Aus- } \\
\text { führung und } \\
\text { Entwicklung } \\
\text { von Methoden }\end{array}$ & $\begin{array}{l}\text { Interpretation } \\
\text { der } \\
\text { Ergebnisse }\end{array}$ & $\begin{array}{l}\text { Konzept und } \\
\text { Schreiben des } \\
\text { Artikels }\end{array}$ \\
\hline$>90 \%$ & $>75 \%$ & $>75 \%$ & $90 \%$ & $>75 \%$ & $>75 \%$ \\
\hline
\end{tabular}

Dieser Beitrag ist so oder in ähnlicher Fassung veröffentlicht in der wissenschaftlichen Zeitschrift ,Berichte über Landwirtschaft" 97 (3). 


\section{Inhalt}

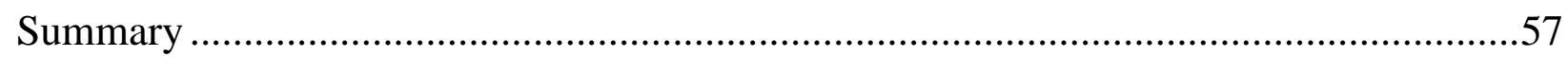

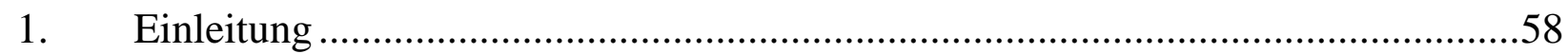

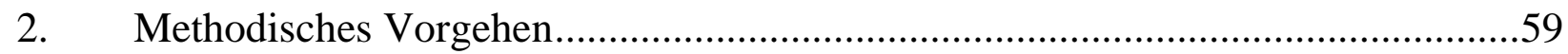

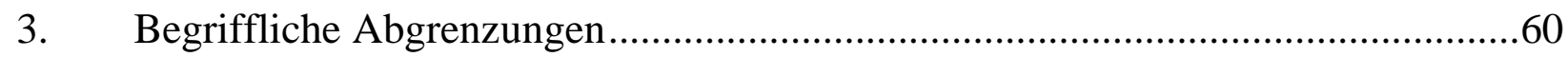

4. Einsatz autonomer Feldroboter im Ackerbau .....................................................62

5. Stand der Akzeptanzforschung .........................................................................64

5.1 Stand der Akzeptanzforschung von Precision Agriculture Technologien ............64

5.2 Akzeptanzforschung zu autonomen Feldrobotern ............................................68

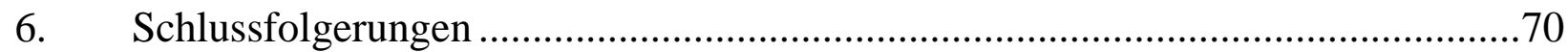

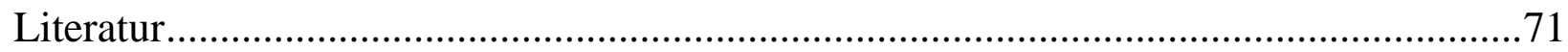




\section{Zusammenfassung}

Autonome Technologiekonzepte sind auf dem Vormarsch. Neben der Automobilindustrie und einigen anderen Branchen erfährt auch die Landwirtschaft als potenzielles Einsatzgebiet zunehmend Aufmerksamkeit, was Fragen zur Technologieakzeptanz aufwirft. Zur Beantwortung dieser Fragen bedarf es zunächst eines Überblicks über den Stand der Forschung. Auf Basis einer umfassenden Literaturrecherche skizziert dieser Beitrag zunächst exemplarisch den aktuellen Einsatz autonomer, bodengebundener Feldroboterkonzepte im Ackerbau. Es folgt ein Überblick über die Forschung zur Akzeptanz von Precision Agriculture Technologien (PAT) und AFR, die unter PAT subsumiert werden können. Insgesamt wurden 35 wissenschaftliche Beiträge zu landwirtschaftlichen Feldrobotern und 11 wissenschaftliche Beiträge aus dem Bereich der Akzeptanzforschung als besonders relevant eingestuft und analysiert. Als Ergebnis zeigte sich, dass die Erforschung der Akzeptanz von AFR Konzepten bisher kaum Beachtung fand, während andere PAT, wie GPSgestützte Lenksysteme oder die teilflächenspezifische Ausbringung von Dünger oder Pflanzenschutzmitteln bereits wiederholt Gegenstand entsprechender Untersuchungen waren. Die Ergebnisse zeigen vielfältige Anknüpfungspunkte für weitere Forschungsarbeiten auf. Besonders interessant wäre in diesem Zusammenhang eine vergleichende Betrachtung der Akzeptanz von AFR Konzepten mit der Akzeptanz bisheriger, mehr oder weniger etablierter PAT in Deutschland.

\section{Summary}

Autonomous technology concepts are on the rise. In addition to the automotive industry and some other industries agriculture is also receiving increasing attention as a potential area of application. That raises questions about technology acceptance. In order to answer these questions an overview of the state of research is needed. This was done in this article on the basis of a comprehensive literature review. It outlines first the current use of autonomous, ground-based field robot concepts in arable farming. The following is an overview of the research on the acceptance of precision agriculture technologies (PAT) and autonomous field robots (AFR), which can be subsumed under PAT. A total of 35 scientific contributions to agricultural field robots and 11 scientific contributions to the field of acceptance research were classified as particularly relevant and analyzed. As a result, research on the acceptance of AFR concepts has received little attention so far while other PAT such as GPS-based steering systems or the site-specific application of fertilizers or pesticides have been repeatedly the subject of research. The results show manifold starting 
points for further research. Particularly interesting in this context would be a comparative analysis of the acceptance of AFR concepts with the acceptance of existing, more or less established PAT in Germany.

\section{Einleitung}

Landwirtschaftliche Betriebe sehen sich aktuell mit vielen Problemen konfrontiert, für die AFR Konzepte einen künftigen Lösungsansatz darstellen könnten. Für Marktfruchtbetriebe in Deutschland zählen dazu bspw. ein zum Teil dem demographischen Wandel geschuldeter Fachkräftemangel, der gesellschaftlich kritisierte Einsatz von Pflanzenschutzmitteln, die sich aufgrund zunehmender Resistenzen aus einem immer kleiner werdenden Spektrum wirksamer Komponenten zusammensetzen, eine verstärkt den landwirtschaftlichen Berufsstand betreffende Diskussion um einen Verlust an Biodiversität in ländlichen Räumen sowie immer größer werdende Landmaschinen, die an die Grenzen der straßenverkehrsrechtlichen Vorgaben stoßen und immensen Druck auf den zu beackernden Boden ausüben (GAUS et al., 2017). AFR können Unkraut mithilfe von Sensoren erkennen und dieses mechanisch (besonders interessant für den Ökolandbau) oder mittels präziser Ausbringung von Pflanzenschutzmitteln bekämpfen (HEUSER et al., 2018). Darüber hinaus wären AFR in der Lage, verschiedene Kulturen auf einem Schlag anzupflanzen und zu beackern, je nach Bodenbeschaffenheit und Nährstoffversorgung. Aus dieser auch als ,spot farming“ bekannten Anbauweise können sich in bestimmten Pflanzenkombinationen Synergieeffekte für die Kulturen ergeben (GAUS et al., 2017). Diese Anbauweise hätte eine positive Wirkung auf die Biodiversität und würde das Landschaftsbild heterogener gestalten, als es bisher mitunter der Fall ist (WEGENER et al., 2018). Durch die kleinen Ausmaße und das relativ geringe Gewicht der Roboter würden sich straßenverkehrsrechtliche Hürden und eine zu hohe Bodenverdichtung als Probleme erübrigen. Zudem könnten durch den geringeren Arbeitskräftebedarf Kosten eingespart werden, die vor allem in arbeitskraftintensiven Bereichen, wie dem Obst- und Gemüseanbau, zum Tragen kommen würden.

KING (2017) spricht AFR ein disruptives Potenzial zu, da diese je nach Umsetzung zu einer kompletten Umwälzung bisheriger Produktionssysteme in der Landwirtschaft führen können. Doch genau das könnte in der Landwirtschaft auf Widerstände stoßen, da AFR, die in eine zunehmend digitalisierte Umwelt eingebunden sind, langfristig gesehen den eigenen Berufstand sukzessive ersetzen bzw. in eine Nebenrolle drängen könnten (DEVITT, 2018; HILLERBRAND et al., 2019). 
Ob sich AFR auf mittlere Sicht etablieren werden, ist gegenwärtig noch völlig offen (GAUS et al., 2017). Eine Grundvoraussetzung für den erfolgreichen Einsatz dieser neuen Technologie ist ihre Akzeptanz durch die Gesellschaft, aber auch die einzelner Stakeholder, wie bspw. die Landwirte oder die Landmaschinenhersteller, damit es bei einem Erstkontakt nicht zur Ablehnung und dem damit verbundenen Scheitern von AFR kommt (BRAUER, 2017). Ein Überblick über die bisherigen wissenschaftlichen Erkenntnisse auf dem Gebiet der Akzeptanzforschung zu AFR fehlte bislang. Ziel dieses Beitrags ist es daher, zunächst die für die hier betrachtete Fragestellung relevanten Begriffe (Autonomisierung, Automatisierung und Feldroboter) präzise abzugrenzen und den derzeitigen Einsatz von AFR im Ackerbau auf Basis der vorhandenen wissenschaftlichen Literatur zu skizzieren. Daran schließt sich ein Forschungsüberblick der Akzeptanz von PAT im Allgemeinen, gefolgt von der Akzeptanz von AFR im Speziellen. Diese können unter PAT subsumiert werden. Davon ausgehend, wird abschließend ein kurzes Fazit gezogen und weiterer Forschungsbedarf aufgezeigt.

\section{Methodisches Vorgehen}

Um dem Anspruch einer umfassenden Recherche zur Darstellung des aktuellen Standes der Literatur zur Akzeptanz von PAT und dann spezieller von AFR in der Landwirtschaft gerecht zu werden, wurde die Fachliteratur der letzten 10 Jahre betrachtet. Die Idee, vollautomatisierte Maschinen in der Landwirtschaft einzusetzen, ist zwar um einiges älter, doch hat sie erst in der jüngsten Vergangenheit an Relevanz gewonnen, was nicht zuletzt auf den Megatrend Digitalisierung zurückgeführt wird (SHAMSHIRI et al., 2018). Die Suche wurde anhand dreier Hauptkriterien eingeschränkt: zum einen wurden nur vollständige wissenschaftliche Artikel berücksichtigt, die Peer-Review-Begutachtungsverfahren durchlaufen hatten; zum anderen musste ein Bezug zur gestellten Forschungsfrage bestehen. Schließlich lag die Konzentration speziell auf ackerbaulichen, bodengebundenen autonomen Roboteranwendungen, da diese in den letzten Jahren sehr viel Aufmerksamkeit erfahren haben.

Aus der Betrachtung ausgeschlossen wurden Artikel, die in einer anderen Sprache als Deutsch oder Englisch veröffentlicht wurden, sowie Beiträge, die sich auf rein technische Fragestellungen begrenzten (z.B. bestimmte Software- oder Sensorteilfragen).

Da ein Literaturüberblick nach BOOTE und BEILE (2005), die sich wiederum auf HART (1999) beziehen, mehreren Anforderungen genügen muss, um den Anspruch der Vollständigkeit erheben zu dürfen, wurde ein strukturiertes, zweistufiges Vorgehen bei der Suche und Analyse der Ergebnisse gewählt (MAXWELL, 2006). Auf der ersten Stufe 
wurden 160 wissenschaftliche Journale, die in dem von DABBERT et al. (2009) veröffentlichtem GEWISOLA-ÖGA Publikationsranking als agrarökonomisch bedeutsam bewertet wurden, nach allen Kombinationsmöglichkeiten zweier Begriffsgruppen durchsucht. Die eine Gruppe bestand aus Begriffen, die sich um autonome, mobile Roboter und digitale Technologien drehen (z.B. autonome Roboter, Digitalisierung, GPS, Kleinmaschinen, smart robots). Die andere Gruppe umfasste Begrifflichkeiten, die zunächst einen Bezug zur Landwirtschaft und dann spezieller zum Ackerbau herstellen (z.B. Landwirtschaft, Feld, precision agriculture, farming). Die Suche wurde anschließend auf größere Plattformen, wie Google Scholar oder Web of Science, aber auch auf die Online-Suchfunktionen diverser Universitätsbibliotheken ausgeweitet, da solche Suchmaschinen eine breite Abdeckung einschlägiger wissenschaftlicher Literatur bieten und über erweiterte bibliometrische Funktionen verfügen, wie bspw. das Vorschlagen von zum Suchbegriff passender Literatur. Die gefundenen Artikel wurden anschließend nach ihrer Relevanz zur Forschungsfrage gesichtet und sortiert.

Auf der zweiten Stufe wurden die als relevant bzw. sehr relevant klassifizierten Artikel analysiert, um die zur Beantwortung der Forschungsfrage wichtigen Informationen zu extrahieren. Dabei wurden weitere, in den analysierten Veröffentlichungen zitierte, relevante Artikel gefunden und hinzugezogen. Als Ergebnis wurden 35 wissenschaftliche Beiträge zu landwirtschaftlichen Feldrobotern und 11 wissenschaftliche Beiträge aus dem Bereich der Akzeptanzforschung als zur Zielerreichung dieses Beitrags besonders relevant eingestuft.

\section{Begriffliche Abgrenzungen}

In Publikationen zu AFR in der Landwirtschaft werden die beiden Begriffe automatisiert und autonom oftmals synonym verwendet. Dabei gilt es hier scharf zu trennen; umso mehr, wenn Fragen der Maschinenethik oder der Technikakzeptanz diskutiert werden.

Nach OETKER (1956) bedeutet Automatisierung, ,, (...) eine ursprünglich durch menschliche Beobachtung, Überlegung und Handlung ausgeführte Folge von Vorgängen zwangsläufig nach einem festgelegten Programm mit technischen Mitteln zu bewirken.". VOIGT (2019) fasst den Begriff etwas weiter, indem er Automatisierung als: „, [Die] Übertragung von Funktionen des Produktionsprozesses, insbesondere Prozesssteuerungs- und -regelungsaufgaben vom Menschen auf künstliche Systeme“ definiert. Beide Begriffsfassungen haben gemein, dass sie sich ausschließlich auf die technischen Aspekte konzentrieren. Die Landwirtschaft durchläuft seit Beginn der Industrialisierung in nahezu allen 
Teilbereichen technische Automatisierungsprozesse, die in zunehmendem Tempo erfolgen. Diese wurden vor allem mit dem Ziel steigender Arbeitsgeschwindigkeit und damit Produktivität vorangetrieben (OETKER, 1956; CHOI et al., 2015). Automatisierte Maschinen treffen keine eigenständigen Entscheidungen, sondern folgen lediglich vorher festgelegten Pfaden bzw. Programmierungen. Laut CHRISTEN (2004) wird erst dann von Maschinenautonomie gesprochen, wenn ein System auf Grundlage einer inneren Dynamik und der Interaktion mit seiner Umwelt agiert. Dafür muss es auf Grundlage von sensorischem Input planen, schlussfolgern und Konsequenzen abschätzen. Zusätzlich müssen die Systeme lernfähig und in der Lage sein, vorgegebene Überwachungs- und Planungsschritte zur Problemlösung selbstständig zu kombinieren sowie mit anderen autonomen Systemen zu kommunizieren.

BeCHAR und Vigneault (2016) definieren Roboter als perzeptive Maschinen, die in Echtzeit handeln und zur Ausführung bestimmter Aufgaben und zum Treffen von Entscheidungen programmiert werden können. Eine Definition für AFR stammt von HERTZBERG (2014). Nach seiner Auffassung sind AFR: ,, (...) Roboter, die in einer automatisierungsaversen Umgebung, also einer nicht kontrollierten und nicht vollständig bekannten Umgebung, entsprechend automatischen Interpretationen von Sensordaten aus dieser Umgebung selbstständig zielgeleitet agieren ".

Die autonome Feldrobotik ist Gegenstand verschiedener Forschungsbereiche. So wird einerseits erforscht, wie sich die Maschinen anhand verschiedener Sensoren in einer nicht kontrollierbaren Umgebung selbstständig lokalisieren. Andererseits wird untersucht, wie die mobilen Roboter ihre Umgebung erfassen und interpretieren, um schließlich Aufgaben zu planen und umzusetzen (HART, 1999). BECHAR und VIGNEAULT (2016) unterteilen die Einsatzfelder der Robotik anhand der Strukturiertheit der Umwelt und der sich darin befindenden Objekte in vier Gruppen (Tabelle 1).

Tabelle 1: Unterteilung Robotik nach Einsatzgebiet

\begin{tabular}{ll|ll}
\hline Einsatzgebiete Robotik & & $\begin{array}{l}\text { Objekte } \\
\text { Strukturiert }\end{array}$ & Unstrukturiert \\
\hline \multirow{2}{*}{ Umwelt } & z.B. Industrie & z.B. Medizin \\
\cline { 2 - 4 } & Strukturiert & z.B. Raumfahrt & z.B. Feldroboter \\
\hline
\end{tabular}

Quelle: Eigene Darstellung nach BECHAR und VIGNEAULT (2016)

Feldroboter fallen in die vierte Gruppe, in der weder die Umwelt noch die Objekte strukturiert sind. Daraus resultieren hohe Ansprüche an die praktische Umsetzung sowie die anschließende Kommerzialisierung solcher Roboterkonzepte (FLETCHER et al., 2005). 


\section{Einsatz autonomer Feldroboter im Ackerbau}

In der Landwirtschaft gibt es Bereiche, in denen vollautomatisierte und teilautonome Systeme, bereits seit einigen Jahren im Einsatz sind (ROLDÁN et al., 2018). Diese wurden allerdings in erster Linie als großtechnische Lösungen konzipiert. Beispiele sind Melkroboter, automatische Futtersysteme aber auch GPS-gestützte Lenksysteme sowie mit Sensoren bestückte Anbaugeräte, die auf Reihenfrüchte und Obstplantagen spezialisiert wurden (bspw. John Deere iTEC Pro oder Claas autonomous navigation). Ziel ist es dabei stets, menschliche Arbeitskraft durch präziser und zuverlässiger arbeitende Technik zu ergänzen oder zu ersetzen, um auf diese Weise mit geringeren Kosten effizienter qualitativ höherwertigere Lebensmittel zu produzieren (SHAMSHIRI, 2013; BAC et al., 2016). Erst in Teilen marktreif bzw. noch im Forschungsstadium sind dagegen vollautonome Systeme, wie mobile Feldroboter, die durch den Einzug der Digitalisierung in die Landwirtschaft vermehrt Aufmerksamkeit erfahren (SHAMSHIRI et al., 2018). So wird ihnen schon jetzt eine tragende Rolle innerhalb des Sektors vorhergesagt (WOLFERT et al., 2017; ChLINGARYAN et al., 2018). Nach KIM und SHIM (2003) und XIANG et al. (2014) bleiben die bisher realisierten autonomen Roboterkonzepte im Praxistest allerdings nicht selten hinter den Erwartungen zurück, weshalb BECHAR und VIGNEAULT (2016) einen wirtschaftlich sinnvollen Einsatz von AFR erst bei entsprechend niedrigen Produktionskosten für gegeben erachten. Die erschwerten Bedingungen durch unterschiedliche Witterungs- und Bodenbedingungen gekennzeichnete Jahreszeiten sowie kurze, intensive Produktionsphasen erfordern möglichst robuste AFR (NOF, 2009). Um dies zu gewährleisten, sind die Forschungsansätze für Feldoder Agrarroboter sehr vielfältig, wie auch die landwirtschaftliche Produktion selbst durch eine große Bandbreite unterschiedlichster Anwendungsmöglichkeiten gekennzeichnet ist (SCHUELLER, 2006).

In der gesichteten Literatur werden AFR nach Arbeitsgang (Bonitur, Pflanzenschutz, Ernte, Auslese usw.), Art (Flug- oder Bodenroboter) oder Ausstattung (Manipulator, Antrieb usw.) unterteilt (BECHAR und VignEAULT, 2016; GAUS et al., 2017; ROLDÁN et al., 2018; SHAMSHIRI et al., 2018). Dabei ist eine klare Zuordnung der Roboter zu einem Arbeitsgang nicht immer möglich, da diese oft mehrere Verfahren ausführen oder als multifunktionale Plattform mit verschiedenen Modulen ausgestattet werden können.

BECHAR und VigneAUlT haben 2016 eine Forschungsübersicht der letzten 30 Jahre im Bereich der technischen Fähigkeiten landwirtschaftlicher Feldroboter, sortiert nach Kulturpflanzen und Arbeitsgang, erstellt. Sie konstatieren, dass es noch einen sehr großen Forschungsbedarf in Hinblick auf die komplexe Umwelt von Feldrobotern gibt, bevor diese erfolgreich in großem Umfang eingesetzt werden können. Obwohl Feldfrüchte, anders als 
bspw. Automobile, zu deren Produktion seit längerem Roboter eingesetzt werden, durch eine relativ geringe Rente ausgezeichnet sind, kann sich der Einsatz von AFR aufgrund stetig sinkender Stückkosten, etwa der benötigten Sensoren, künftig dennoch als ökonomisch vorteilhaft darstellen (BECHAR und VIGNEAULT, 2016). Daneben betrachten sie die Sicherheit und Zuverlässigkeit solch neuartiger Technologien als wichtige Aspekte, damit ihr Einsatz in einer unkontrollierten Umgebung, wie etwa einer Ackerfläche, von den Stakeholdern akzeptiert wird.

Einen Überblick über AFR Konzepte für die Arbeitsgänge Bodenbearbeitung, Aussaat, Bonitur, Pflege und Ernte sowie verfahrensübergreifende Arbeiten, die kurz vor einer Markteinführung stehen oder bereits realisiert wurden, vermittelt der Abschlussbericht von GAUS et al. (2017). Sie merken an, dass bisher überwiegend Roboterkonzepte als Teillösungen für einzelne Aufgaben entwickelt und nicht etwa umfassende Pflanzenbausysteme als Ganzes betrachtet wurden. Folglich plädieren sie für eine stärkere Vernetzung bestehender Insellösungen, damit die ökologischen Potenziale von AFR Konzepten zum Tragen kommen können.

RoLDÁN et al. (2018) umreißen in ihrer Studie den Status quo der Entwicklung von Luft-, Boden- und Spezialfeldrobotern sowie von Roboterschwärmen. Sie stellen fest, dass bisher vor allem Drohnen in der Landwirtschaft zum Einsatz kommen und attestieren ihnen in Kombination mit Bodenroboterschwärmen ein hohes Potenzial für die Zukunft.

Eine ausführliche Übersicht über die jüngsten Entwicklungen von Feldroboterkonzepten zur Unkrautkontrolle und Einzelpflanzenbehandlung, zur Bonitur und Feldkartierung sowie zur Gemüse- und Obsternte haben SHAMSHIRI et al. (2018) erstellt. Sie kommen zu dem Schluss, dass bisherige Roboterkonzepte noch nicht weit genug entwickelt wurden, um menschliche Arbeitskräfte in der Landwirtschaft zu substituieren. Dafür müssten Arbeitsgeschwindigkeit und Präzision der Feldroboter gesteigert werden, weshalb sie sich in diesem Zusammenhang für Schwarmtechnologien, die aus der Luft durch Drohnen mit Informationen unterstützt werden, aussprechen. 


\section{Stand der Akzeptanzforschung}

Trotz der bereits durchführbaren Arbeitsgänge und der zahlreichen Vorteile, die autonome Roboterkonzepte prinzipiell mit sich bringen, kommt ihre Kommerzialisierung eher schleppend voran (SHAMSHIRI et al., 2018). Die Einflussfaktoren auf die Akzeptanz und damit einhergehend die weitere Verbreitung von AFR haben in der Forschung bisher kaum Beachtung gefunden (DEVITT, 2018; RIAL-LOVERA, 2018).

Sehr viel zahlreicher sind dagegen Studien zur Akzeptanz von PAT, unter die mobile Feldroboter subsumiert werden können (Tabelle 2). Daher werden im Abschnitt 5.1 zunächst ausgewählte Studien zur Adoption von PAT chronologisch vorgestellt, woran sich im Abschnitt 5.2 eine Übersicht über die wenigen bisher durchgeführten Studien zur Akzeptanz von AFR Konzepten anschließt.

\subsection{Stand der Akzeptanzforschung von Precision Agriculture Technolo- gien}

AUbert et al. (2012) haben die Akzeptanzhemmnisse von PAT in der Provinz Quebec (Kanada) untersucht. Dafür wurden 1.998 Fragebögen an dort ansässige Landwirte verschickt, von denen 438 Fragebögen ausgewertet werden konnten. Die Fragen bezogen sich auf den Einsatz von GPS, geographischen Informationssystemen (GIS), Erntekartierung, Pflanzenerkennung und Fernerkundung, Teilflächenapplikation, Autopiloten und Navigation. Als Ergebnis zeigte sich, dass die Akzeptanz von PAT sowohl von der wahrgenommenen Benutzerfreundlichkeit als auch dem empfundenen Nutzen abhängig ist und durch mangelnde Kompatibilität sowie durch fehlendes Wissen um den Nutzen von PAT für Landwirte gehemmt wird. Im Gegensatz zu anderen Studien hatte in der Untersuchung von AUBERT et al. (2012) weder das Alter des Landwirtes noch die Größe des Betriebes einen Einfluss auf die Akzeptanz der untersuchten Technologien.

CASTLE et al. (2016) untersuchten die Akzeptanz verschiedener PAT sowie die Nutzung von Smartphones durch Landwirte in Nebraska (USA), die Reihenkulturen beackern. Die Antworten der 102 befragten Landwirte wurden mithilfe eines Poisson-Modells auf die Akzeptanz beeinflussende Faktoren hin analysiert. Als Ergebnis zeigte sich, dass größere Landwirtschaftsbetriebe eher verschiedene PAT einsetzen als kleinere. Zudem wurde deutlich, dass Landwirte, die ein Smartphone mit Internetzugang benutzen, eher bereit sind, PAT einzusetzen.

SCHIMMELPFENNIG und EBEL (2016) gingen der Frage nach, ob sich bestimmte Verhaltensmuster der Landwirte hinsichtlich der Akzeptanz von PAT (v.a. in Hinblick auf 
Teilflächenmanagement) ermitteln lassen und ob der Einsatz solcher Technologien tatsächlich ökonomisch vorteilhafter ist als herkömmliche Technologien. Dafür wurde ein Behandlungseffektmodell entwickelt, dessen Grundlage die Daten einer groß angelegten Erhebung (Agricultural Resource Management Survey - ARMS) des U.S. Department of Agriculture (USDA) aus dem Jahr 2010 bildeten $(n=1.507)$. Es stellte sich heraus, dass vor allem größere Betriebe mit besser ausgebildeten Arbeitskräften, die sowohl den bewirtschafteten Boden regelmäßig auf Nährstoffe untersuchen als auch genverändertes Saatgut einsetzen, bevorzugt als „Early Adopters“ von PAT in Erscheinung treten. Daneben fanden SCHIMMELPFENNIG und EBEL (2016) heraus, dass die höchsten Kostenersparnisse erzielt werden konnten, wenn die beiden PAT Boden- und Erntekartierung kombiniert wurden.

TORREZ et al. (2016) gingen der Frage nach, welche Faktoren die Akzeptanz von PAT durch Landwirte in Kansas (USA) beeinflussen und wie sich die PAT einsetzenden Landwirte charakterisieren lassen. Dafür wurden Landwirte, die gleichzeitig Mitglieder der Kansas Farm Management Association (KFMA) sind, mittels Fragebogen befragt $(n=453)$. Die Erhebung wurde anschließend mithilfe eines Adoption Decision Model analysiert. Wie auch in der Studie von SCHIMMELPFENNIG und EbEL (2016), korrelierte die Betriebsgröße positiv mit der PAT-Akzeptanz durch die Betriebsleiter. Darüber hinaus zeigte sich, dass die Einschätzung der ökonomischen Vorteilhaftigkeit des PAT-Einsatzes mit zunehmendem Alter des Landwirtes abnahm. Zusätzlich wurde bestätigt, dass der Faktor Zeit einen positiven und statistisch signifikanten Einfluss auf die Akzeptanz von PAT und deren Verbreitung hat. Dabei erfasst der Diffusionsfaktor Zeit alle Wechselwirkungen, die durch das über die Zeit sich stetig verbessernde PAT Knowhow und fortschreitende Technologieentwicklungen entstehen und schwer messbar sind.

ERICKSON et al. (2017) befragten landwirtschaftliche Dienstleister in den USA zu verschiedenen PAT $(n=209)$. Ziel war es, ein besseres Verständnis des praktischen Einsatzes sowie der Akzeptanzhemmnisse von PAT und deren Rentabilität zu erlangen. In den letzten Jahren kam es demnach zu einer verstärkten Nutzung von Technologien zur Datenerhebung, wie Stickstoffsensoren, drohnengestützten Kartierungen und Leitfähigkeitsmessungen des Bodens, während der Einsatz von Telemetriesystemen abnahm. Als Akzeptanzhemmnisse wurden das Einkommen der Landwirte und der erwartete, als zu gering bewertete Nutzen von PAT identifiziert. Daneben gaben die Dienstleister an, dass sie Hard- und Softwareinkompatibilitäten, zu hohe Produktpreise und das Aufzeigen eines Mehrwertes von PAT für die Landwirte als Herausforderung erachten.

In dem Beitrag von ZHOU et al. (2017) ging es um Entwicklungstendenzen und geografische Muster der Akzeptanz von PAT unter Baumwollanbauern in 14 südlichen US- 
Bundesstaaten. Zu diesem Zweck wurden Fragebögen per Mail an Baumwollanbauer verschickt. Die verwertbaren und in die weitere Analyse eingeflossenen Antworten beliefen sich auf 1.811 der 13.566 verschickten Fragebögen. Es konnte gezeigt werden, dass 73,5 \% der Befragten mindestens eine PAT einsetzten. Im Einzelnen nutzten 40,9 \% der Befragten Datenerhebungsinstrumente (wie automatische Ernte- oder Bodenkartierung), 67 \% GPSFührung, 25,3 \% variable Mengenausbringung und 29,3\% automatische Lenkfunktionen. Die höchste PAT-Akzeptanz wurde im Corn Belt, gefolgt vom Mississippi Delta und den Northern und Southern Plains festgestellt. Der geringste PAT-Einsatz erfolgte in den südwestlichen Regionen.

Mittels einer Medienanalyse, in die 210 Artikel aus drei Fachzeitschriften, die zwischen 2009 und 2016 veröffentlicht wurden, einflossen, untersuchten SCHLEICHER und GRANDORFER (2018), welche Gründe der Akzeptanz von PAT in Deutschland entgegenstehen. In absteigender Häufigkeit wurden folgende Akzeptanzhemmnisse genannt: hoher Investitionsbedarf, Sorge um Datenschutz und Inkompatibilität verschiedener Systeme.

THOMPSON et al. (2018) untersuchten die Bewertung von vier PAT (teilflächenspezifische Düngung, GPS-gestützte Bodenproben, automatisches Lenken und Erntekartierung) durch US-amerikanische Landwirte, die mehr als 1.000 Acres (ca. 405 Hektar) konventionell bewirtschaften. Von den 5.295 via Telefon kontaktierten Landwirten vollendeten 837 Landwirte die Umfrage vollständig, sodass diese analysiert werden konnten. Zusätzlich wurde ein paarweises Best-Worse-Scaling Choice Experiment durchgeführt, um die präferierten PAT-Vorteile der Befragten zu ermitteln. Das Ergebnis stellte sich heterogen dar. So wurde die größten Vorteile in der Wahrnehmung von PAT unter den befragten Landwirten entweder in der Ertragssteigerung oder in der Kostenreduktion und gesteigerten Bequemlichkeit durch die Nutzung von PAT gesehen. Die Wahrnehmung der Befragten wurde dabei nachweislich von den PAT beeinflusst, die sie selbst nutzen. 
Tabelle 2: Literaturüberblick zur Akzeptanzforschung von PAT

\begin{tabular}{|c|c|c|c|}
\hline Autoren (Jahr) & Forschungsfrage & Methode & Zentrale Ergebnisse \\
\hline $\begin{array}{l}\text { AUBERT et } \\
\text { al. (2012) }\end{array}$ & $\begin{array}{l}\text { Akzeptanzhemm- } \\
\text { nisse von PAT in der } \\
\text { Provinz Quebec (Ka- } \\
\text { nada) }\end{array}$ & $\begin{array}{l}\text { Umfrage } \\
\text { Landwirte } \\
\text { Kanada } \\
(n=438)\end{array}$ & $\begin{array}{l}\text { - Akzeptanz abhängig von wahrgenom- } \\
\text { mener Benutzerfreundlichkeit und emp- } \\
\text { fundener Nutzen von PAT } \\
\text { - Hemmnisse: v.a. mangelnde Kompati- } \\
\text { bilität und fehlendes Wissen um Nutzen } \\
\text { von PAT der Landwirte } \\
\text { - Betriebsgröße und Alter haben keinen } \\
\text { Einfluss auf Akzeptanz von untersuch- } \\
\text { ten PAT }\end{array}$ \\
\hline $\begin{array}{l}\text { CASTLE et al. } \\
(2016)\end{array}$ & $\begin{array}{l}\text { Faktorenanalyse der } \\
\text { Akzeptanz von PAT } \\
\text { und Smartphonenut- } \\
\text { zung unter Reihen- } \\
\text { pflanzenanbauern in } \\
\text { Nebraska (USA) }\end{array}$ & $\begin{array}{l}\text { Umfrage } \\
\text { Landwirte } \\
\text { Nebraska } \\
(n=102) ; \\
\text { Poisson-Mo- } \\
\text { dell }\end{array}$ & $\begin{array}{l}\text { - Je größer der Landwirtschaftsbetrieb, } \\
\text { desto höher ist die Akzeptanz von PAT } \\
\text { - Wenn ein Anbauer über ein Mobiltele- } \\
\text { fon mit Internetzugang verfügt, ist die } \\
\text { Nutzung von PAT wahrscheinlicher }\end{array}$ \\
\hline $\begin{array}{l}\text { SCHIMMEL-PFEN- } \\
\text { NIG und EBEL } \\
\text { (2016) }\end{array}$ & $\begin{array}{l}\text { Welche Verhaltens- } \\
\text { muster stecken hinter } \\
\text { der Akzeptanz von } \\
\text { PAT (v.a. im Hin- } \\
\text { blick auf variable } \\
\text { Mengenausbringung) } \\
\text { durch Landwirte und } \\
\text { sind PAT tatsächlich } \\
\text { ökonomisch sinn- } \\
\text { voll? }\end{array}$ & $\begin{array}{l}\text { Behandlungs- } \\
\text { effektmodell } \\
\text { auf Grundlage } \\
\text { einer Umfrage } \\
\text { (USDA) } \\
(n=1.507)\end{array}$ & $\begin{array}{l}\text { - PAT werden eher von größeren Betrie- } \\
\text { ben/Landwirten mit höherem Bildungs- } \\
\text { abschluss bevorzugt } \\
\text { - Teilflächenapplikation führt zu größten } \\
\text { Kosteneinsparungen, wenn sie mit Bo- } \\
\text { denkartierung (und nicht allein der Er- } \\
\text { tragskartierung) kombiniert wird }\end{array}$ \\
\hline $\begin{array}{l}\text { TORREZ et al. } \\
\text { (2016) }\end{array}$ & $\begin{array}{l}\text { Identifikation von } \\
\text { Einflussfaktoren der } \\
\text { Akzeptanz von PAT } \\
\text { und Charakterisie- } \\
\text { rung der PAT einset- } \\
\text { zenden Landwirte in } \\
\text { Kansas (USA) }\end{array}$ & $\begin{array}{l}\text { Umfrage } \\
\text { Landwirte } \\
\text { (KFMA Mit- } \\
\text { glieder) Kan- } \\
\text { sas }(n=453) \\
\text { Adoption De- } \\
\text { cision Modell }\end{array}$ & $\begin{array}{l}\text { - Je größer der Landwirtschaftsbetrieb, } \\
\text { desto höher ist die Akzeptanz von PAT } \\
\text { - Jüngere Landwirte akzeptieren PAT } \\
\text { eher als ältere } \\
\text { - Diffusionsfaktor Zeit hat einen positi- } \\
\text { ven Einfluss auf Akzeptanz von PAT }\end{array}$ \\
\hline $\begin{array}{l}\text { ERICKSON et } \\
\text { al. (2017) }\end{array}$ & $\begin{array}{l}\text { Verständnis von } \\
\text { Praktiken und Ein- } \\
\text { schränkungen bei der } \\
\text { Akzeptanz und Ren- } \\
\text { tabilität von PAT }\end{array}$ & $\begin{array}{l}\text { Umfrage } \\
\text { PAT-Dienst- } \\
\text { leister USA } \\
(n=209)\end{array}$ & $\begin{array}{l}\text { - Zunahme des Einsatzes von unbemann- } \\
\text { ten Fahrzeugen (z.B. Drohnen) zur Da- } \\
\text { tenerhebung bei Dienstleistern und } \\
\text { Landwirten } \\
\text { - Abnahme des Einsatzes von Telemet- } \\
\text { riesystemen } \\
\text { - Akzeptanzhemmnisse: zu geringes Ein- } \\
\text { kommen der Landwirte bzw. zu hohe } \\
\text { Produktpreise, Nutzen von PAT, man- } \\
\text { gelnde Hard- und Softwarekompatibili- } \\
\text { tät }\end{array}$ \\
\hline $\begin{array}{l}\text { ZHOU et al. } \\
\text { (2017) }\end{array}$ & $\begin{array}{l}\text { Entwicklungstenden- } \\
\text { zen und geografische } \\
\text { Muster der Akzep- } \\
\text { tanz von PAT unter } \\
\text { Baumwollanbauern } \\
\text { in } 14 \text { südlichen } \\
\text { US-Bundesstaaten } \\
\end{array}$ & $\begin{array}{l}\text { Umfrage } \\
\text { Landwirte } \\
\text { USA } \\
(n=1.811)\end{array}$ & $\begin{array}{l}\text { - 73,5\% der Befragten nutzten mindes- } \\
\text { tens eine PAT } \\
\text { - Im Detail nutzten 40,9\% Datenerhe- } \\
\text { bungsinstrumente, } 67 \% \text { GPS-Führung, } \\
\text { 25,3\% variable Mengenausbringung, } \\
29,3 \% \text { automatische Lenkfunktion }\end{array}$ \\
\hline
\end{tabular}




\begin{tabular}{|c|c|c|c|}
\hline & & & $\begin{array}{l}\text { - Höchste PAT-Akzeptanz im Corn Belt; } \\
\text { geringste PAT-Akzeptanz in südwestli- } \\
\text { chen Regionen }\end{array}$ \\
\hline $\begin{array}{l}\text { SCHLEICHER und } \\
\text { GRANDORFER } \\
\text { (2018) }\end{array}$ & $\begin{array}{l}\text { Bedeutung von Ak- } \\
\text { zeptanzhemmnissen } \\
\text { von PAT in Deutsch- } \\
\text { land im Zeitverlauf }\end{array}$ & $\begin{array}{l}\text { Medienanalyse } \\
\text { dreier Fach- } \\
\text { zeitschriften }\end{array}$ & $\begin{array}{l}\text { - Hoher Investitionsbedarf, Sorge um } \\
\text { Datenschutz/Datenhoheit und Inkompa- } \\
\text { tibilität zwischen den Systemen als } \\
\text { häufigste Hemmnisse genannt }\end{array}$ \\
\hline $\begin{array}{l}\text { THOMPSON et al. } \\
\text { (2018) }\end{array}$ & $\begin{array}{l}\text { Bewertung Land- } \\
\text { wirte (USA; > } 1.000 \\
\text { Acres) bestimmter } \\
\text { PAT }\end{array}$ & $\begin{array}{l}\text { Telefonum- } \\
\text { frage } \\
(n=837) ; \\
\text { Best-Worse- } \\
\text { Scaling Choice } \\
\text { Experiment }\end{array}$ & $\begin{array}{l}\text { - Wahrnehmung der Vorteile von PAT } \\
\text { unter Landwirten heterogen } \\
\text { - Meisten Nennungen: Ertragssteigerung, } \\
\text { Kostenreduktion, Bequemlichkeit } \\
\text { - Wahrnehmung abhängig von eigener } \\
\text { PAT-Nutzung }\end{array}$ \\
\hline
\end{tabular}

Quelle: Eigene Darstellung

\subsection{Akzeptanzforschung zu autonomen Feldrobotern}

Zur Akzeptanz von AFR konnten drei relevante Beiträge gefunden werden (Tabelle 3). Im Jahr 2011 analysierte POPA (2011) Roboterkonzepte im Zuge einer umfassenden Betrachtung des Einsatzes von künstlicher Intelligenz (KI) in der Landwirtschaft. Dafür wurden unter anderem 64 verschiedene Online-Expertensysteme (Software) und etwa 200 Videos zu diesem Thema ausgewertet. Als Ergebnis fand er heraus, dass der Einsatz von KI in der Landwirtschaft zwar wächst; Zugang zu diesen Technologien hatten zum Zeitpunkt der Erhebung aber nur etwa $30 \%$ der Weltbevölkerung. Für das Jahr 2030 wurde prognostiziert, dass global $75 \%$ der Landwirte KI nutzen werden. Er kam darüber hinaus zu dem Ergebnis, dass der Einsatz von Robotern und autonomen Geräten in der Landwirtschaft noch in den Kinderschuhen steckt und es mehr Zeit und Investitionen bedürfe, ehe solche Roboterkonzepte fester Bestandteil des landwirtschaftlichen Alltags werden.

DEVITT (2018) untersuchte in ihrer Literaturanalyse kognitive, die Akzeptanz autonomer Roboter- und Landtechnikkonzepte beeinflussende Faktoren. Sie kam zu dem Schluss, dass mangelndes Vertrauen der Landwirte in neuartige Technik, ihre Angst vor dem Verlernen landwirtschaftlicher Fähigkeiten und ihre Sorge vor dem Verlust sozialer Anerkennung wichtige kognitive Einflussfaktoren sind. Daneben konstatierte sie, dass mit zunehmenden Autonomisierungsgrad der Technik neben der kognitiven Belastung auch das Situationsbewusstsein des Landwirts für betriebliche Vorgänge sinkt.

Eine qualitative Studie zur Akzeptanz autonomer Roboterkonzepte in der Landwirtschaft führte RIAL-LOVERA (2018) durch. Sie hat auf der Suche nach Treibern und Barrieren der Akzeptanz von landwirtschaftlichen Robotern und Möglichkeiten zur Akzeptanzförderung 14 Experten befragt. Darunter befanden sich fünf Hersteller von Feldrobotern, vier auf dem Gebiet der Feldrobotik tätige Wissenschaftler, zwei Lebensmittelhersteller, ein 
Landmaschinenhändler und ein Landwirt. Die befragten Experten nannten die mangelnde Verfügbarkeit von Arbeitskräften und die Hoffnung auf sinkende Arbeitserledigungskosten als Haupttreiber der Entwicklung der Robotertechnologie. Im Gegensatz zu Ergebnissen von KUTTER et al. (2011) betrachteten die Experten Fragen des Datenschutzes und der Datenhoheit nicht als Akzeptanzbarrieren. Als Haupthindernisse nannten sie die mit der Anschaffung von AFR verbundenen Kosten sowie die fehlende Komptabilität und Standardisierung der Geräte. Wolle man die Akzeptanz fördern, müssten die Roboterkonzepte Landwirten in Form von praktischen Demonstrationen vorgestellt werden. Zudem würden auch finanzielle Anreize die Akzeptanz fördern.

Tabelle 3: Literaturüberblick zur Akzeptanzforschung von AFR

\begin{tabular}{|c|c|c|c|}
\hline Autoren (Jahr) & Forschungsfrage & Methode & Zentrale Ergebnisse \\
\hline POPA (2011) & $\begin{array}{l}\text { Status quo und } \mathrm{Zu}- \\
\text { kunft des Einsatzes } \\
\text { von KI in der Land- } \\
\text { wirtschaft }\end{array}$ & Literaturanalyse & $\begin{array}{l}\text { - KI-Einsatz wächst } \\
\text { - Prognose 2030: } 75 \% \text { der Landwirte } \\
\text { nutzen KI } \\
\text { - Bisher kaum Einsatz autonomer Robo- } \\
\text { ter in der Landwirtschaft } \\
\text { - Technologie braucht Zeit und Investi- } \\
\text { tionen, um bestehende Technik zu } \\
\text { übertreffen }\end{array}$ \\
\hline DEVITT (2018) & $\begin{array}{l}\text { Kognitive Einfluss- } \\
\text { faktoren auf die Ak- } \\
\text { zeptanz autonomer } \\
\text { Roboter- und Land- } \\
\text { technik }\end{array}$ & Literaturanalyse & $\begin{array}{l}\text { - Wichtige Faktoren: mangelndes Ver- } \\
\text { trauen in neuartige Technik, Angst vor } \\
\text { dem Verlernen landwirtschaftlicher } \\
\text { Fähigkeiten, Verlust von sozialer An- } \\
\text { erkennung } \\
\text { - Je autonomer das System, desto gerin- } \\
\text { ger ist zwar die kognitive Belastung, } \\
\text { doch desto geringer ist auch das Situa- } \\
\text { tionsbewusstsein betrieblicher Vor- } \\
\text { gänge }\end{array}$ \\
\hline $\begin{array}{l}\text { RIAL-LOVERA } \\
\text { (2018) }\end{array}$ & $\begin{array}{l}\text { Treiber und Barrie- } \\
\text { ren der Akzeptanz } \\
\text { landwirtschaftlicher } \\
\text { Roboter und Mög- } \\
\text { lichkeiten zur Ak- } \\
\text { zeptanzförderung }\end{array}$ & $\begin{array}{l}\text { Experteninter- } \\
\text { views } \\
(n=14)\end{array}$ & $\begin{array}{l}\text { - Treiber: Fachkräftemangel, Arbeitser- } \\
\text { ledigungskosten } \\
\text { - Datenschutz und Datenhoheit keine } \\
\text { Akzeptanzbarrieren } \\
\text { - Barrieren: Anschaffungs- und Unter- } \\
\text { haltskosten, fehlende Standardisierung } \\
\text { - Förderung: praktische Demonstratio- } \\
\text { nen und finanzielle Anreize }\end{array}$ \\
\hline
\end{tabular}




\section{Schlussfolgerungen}

Ziel dieses Beitrags war es, in einem ersten Schritt den Einsatz autonomer, bodengebundener Feldroboter im Ackerbau als Teil einer zunehmend automatisierten Landwirtschaft zu skizzieren. In einem zweiten Schritt sollte der Stand der Forschung zur Akzeptanz von PAT unter besonderer Berücksichtigung von AFR Konzepten zusammengetragen werden, um so abschließend bestehenden Forschungsbedarf zu identifizieren.

Es konnte gezeigt werden, dass sich autonome landwirtschaftliche Kleinmaschinen noch in der Forschungs- bzw. einer sehr frühen Markteinführungsphase befinden, wenngleich die Forschungsaktivitäten in jüngerer Zeit stark zugenommen haben (SHAMSHIRI et al., 2018). Während den AFR Konzepten ein großes Potenzial in den Bereichen Bonitur, Feldkartierung, sensorische Datenerfassung und Schädlingsbekämpfung als Teil der Bestandspflege in Feldfrüchten zugesprochen wird, werden autonome Ernteroboterkonzepte bisher nahezu ausschließlich für Obst und Gemüse im Unterglasanbau entwickelt. Mögliche Gründe hierfür können in der großen Anzahl benötigter Arbeitskräfte und den damit einhergehenden Kosten im Obsternteprozess sowie in den sich stark, bspw. von der Getreideernte, unterscheidenden Randbedingungen liegen (BECHAR und EBEN-CHAIME, 2014). So müssen bei der Obsternte einzelne Früchte erkannt und abgetrennt werden, während bei der Ernte von Feldfrüchten die Pflanze als Ganzes geerntet und verarbeitet wird, was andere Anforderungen an ein Roboterkonzept stellt (GAUS et al., 2017).

Während die Akzeptanz von PAT bereits recht umfassend erforscht wurde, konnte in Bezug auf die Akzeptanz von AFR ein großes Forschungsdefizit ermittelt werden. Dieses ist möglicherweise dem bisher marginal ausfallenden Einsatz solcher Systeme in der Praxis geschuldet. Interessant erscheint in diesem Zusammenhang neben der weiteren Erforschung der Akzeptanz von AFR Konzepten eine sich daran anschließende vergleichende Betrachtung mit der Akzeptanz bisheriger, mehr oder weniger etablierter PAT. So könnte sich bspw. die wissenschaftlich mehrfach belegte positive Korrelation zwischen Betriebsgröße und PAT-Einsatz im Falle von AFR anders darstellen, da diese als Roboterschwarm besser skalierbar sind und somit auch für kleinere Betriebe eine interessante Alternative zu bisherigen großtechnischen Lösungen darstellen könnten. Daneben beziehen sich die wenigen bisher durchgeführten Studien zur Akzeptanz von AFR Konzepten vor allem auf den nordamerikanischen Raum. Hier muss überprüft werden, ob sich die gefundenen Ergebnisse auf die deutsche Landwirtschaft übertragen lassen oder ob Unterschiede bestehen. Offen ist bislang auch die Frage geblieben, ob sich ggf. die Wahrnehmungen verschiedener Stakeholdergruppen, wie Landwirten, Landmaschinenherstellern oder der Gesellschaft als Ganzes, unterscheiden. Denn einerseits versprechen Feldroboter 
ökologische Vorteile durch gezieltere Ausbringung bspw. von Dünge- und Pflanzenschutzmitteln, eine bodenschonendere Wirtschaftsweise sowie eine geringere Lärmbelastung; andererseits verfügen AFR auf lange Sicht aber auch über das Potenzial, den Landwirt zunehmend zu ersetzen (HILLERBRAND et al., 2019). Daher sind unterschiedliche Bewertungen der Technologie wahrscheinlich. Ebenso wäre eine vertiefte Analyse möglicher regionaler Unterschiede der Akzeptanz von AFR wünschenswert, um auf diese Weise mögliche Akzeptanzhemmnisse und -treiber aufzudecken und damit die Chancen dafür zu verbessern, dass sich eine vielversprechende Technologie in der Landwirtschaft zu etablieren.

\section{Literatur}

Aubert, B. A., Schroeder, A. und J. Grimaudo (2012): IT as enabler of sustainable farming: An empirical analysis of farmers' adoption decision of precision agriculture technology. In: Decision Support Systems 54 (1): 510-520, doi: 10.1016/j.dss.2012.07.002.

Bac, C. W., RoOrda, T., Reshef, R., Berman, S., Hemming, J. und E. J. van Henten (2016): Analysis of a motion planning problem for sweet-pepper harvesting in a dense obstacle environment. In: Biosystems Engineering 146: 85-97, doi: 10.1016/j.biosystemseng.2015.07.004.

BECHAR, A. und M. EBEN-CHAIME (2014): Hand-held computers to increase accuracy and productivity in agricultural work study. In: International Journal of Productivity and Performance Management 63 (2): 194-208.

BECHAR, A. und C. VigneAult (2016): Agricultural robots for field operations: Concepts and components. In: Biosystems Engineering 149: 94-111, doi: 10.1016/j.biosystemseng.2016.06.014.

Boote, D. N. und P. BEILE (2005): Scholars before Researchers: On the Centrality of the Dissertation Literature Review in Research Preparation. In: Educational Researcher 34 (6): 3-15, doi: 10.3102/0013189X034006003.

BRAuER, R. (2017): Akzeptanz kooperativer Roboter im industriellen Kontext. Universitätsverlag, Chemnitz.

Castle, M. H., LubBen, B. D. und J. D. LuCK (2016): Factors Influencing the Adoption of Precision Agriculture Technologies by Nebraska Producers, Presentations, Working Papers, and Gray Literature: Agricultural Economics Paper 49. URL: http://digitalcommons.unl.edu/ageconworkpap/49 (Abrufdatum: 18.07.2018).

Chlingaryan, A., Sukkarieh, S. und B. Whelan (2018): Machine learning approaches for crop yield prediction and nitrogen status estimation in precision agriculture: A review. In: Computers and Electronics in Agriculture 151: 61-69, doi: 10.1016/j.compag.2018.05.012.

CHOI, K. H., HAN, S. K., HAN, S. H., PARK, K.-H., KIM, K.-S. und S. KIM (2015): Morphology-based guidance line extraction for an autonomous weeding robot in paddy fields. In: Computers and Electronics in Agriculture 113: 266-274, doi: 10.1016/j.compag.2015.02.014.

Christen, M. (2004): Schuldige Maschinen? Autonome Systeme als Herausforderung für das Konzept der Verantwortung. In: HONNEFELDER, L. und C. STREFFER (Hrsg.): Jahrbuch für Wissenschaft und Ethik 9. Walter de Gruyter, Berlin.

Dabbert, S., Berg, E., Herrmann, R., PÖChtrager, S. und K. SAlHOFER (2009): Kompass für agrarökonomische Zeitschriften: Das GEWISOLA-ÖGA-Publikationsranking. Arbeitspapier. In: Agrarwirtschaft 58 (2): 109-113.

DevitT, S. K. (2018): Cognitive Factors that Affect the Adoption of Autonomous Agriculture. In: Farm Policy Journal 15 (2): 49-60. 
ERICKson, B., LOWEnBerg-Deboer, J. und J. Bradford (2017): 2017 Precision Agricultural Service Dealership Survey. URL: http://agribusiness.purdue.edu/files/file/croplife-purdue-2017-precision-dealersurvey-report.pdf (Abrufdatum: 18.07.2018).

FLeTCHER, L., LOY, G., BARNES, N. und A. ZELINSKY (2005): Correlating driver gaze with the road scene for driver assistance systems. In: Robotics and Autonomous Systems 52 (1): 71-84, doi: 10.1016/j.robot.2005.03.010.

Gaus, C. C., Minßen, T. F., Urso, L. M., DE WiTTE, T. und J. Wegener (2017): Mit autonomen Landmaschinen zu neuen Pflanzenbausystemen. Schlussbericht. Johann Heinrich von Thünen-Institut, Bundesforschungsinstitut für Ländliche Räume, Wald und Fischerei; Technische Universität Braunschweig, Institut für mobile Maschinen und Nutzfahrzeuge; Julius-Kühn-Institut, Braunschweig.

HART, C. (1999): Doing a literature review: Releasing the social science research imagination. SAGE, Los Angeles.

HeRTZBERG, J. (2014): Autonome Systeme in der Landwirtschaft. KTBL, Darmstadt.

Heuser, S., Demmel, M., Pfeiffer, J. und M. Gandorfer (2018): Automatisierte mechanische Unkrautregulierung. In: Ruckelshausen, A., Meyer-Aurich, A., Borchardt, K., HofaCKer, C., LOY, J.-P., SchwerdtFeger, R., Sundermeier, H.-H., Floto, H. und B. Theuvsen (Hrsg.): Digitale Marktplätze und Plattformen. Lecture Notes in Informatics 278, Gesellschaft für Informatik, Bonn: 99-102.

Hillerbrand, F., Treiber, M., BAUerdick, J. und H. Bernhardt (2019): Robotik in der Außenwirtschaft - Entwicklungskonzepte und tendenzielle Einflussmöglichkeiten auf die Prozesssteuerung durch den Landwirt. In: Meyer-Aurich, A., GANDOrfer, M., BArta, N., Gronauer, A., KantelHardt, J. und H. FlOTO (Hrsg.): Digitalisierung für landwirtschaftliche Betriebe in kleinstrukturierten Regionen - ein Widerspruch in sich? Lecture Notes in Informatics 287, Gesellschaft für Informatik, Bonn: 77-82.

KIM, H. und D. SHIM (2003): A flight control system for aerial robots: algorithms and experiments. In: Control Engineering Practice 11 (12): 1389-1400, doi: 10.1016/S0967-0661(03)00100-X.

KING, A. (2017): Technology: The Future of Agriculture. In: Nature 544 (7651): 21-23, doi: 10.1038/544S21a.

KutTER, T., TIEMANn, S., SieberT, R. und S. Fountas (2011): The role of communication and co-operation in the adoption of precision farming. In: Precision Agriculture 12 (1): 2-17, doi: 10.1007/s11119-0099150-0.

MAXWELl, J. A. (2006): Literature Reviews of, and for, Educational Research: A Commentary on Boote and Beile's "Scholars before Researchers". In: Educational Researcher 35 (9): 28-31.

NoF, S. Y. (2009): Springer Handbook of Automation. Springer, Berlin/Heidelberg.

OETKER, R. (1956): Was heißt "Automatisieren"? In: Regelungstechnik 9: 210-213.

POPA, C. (2011): Adoption of Artificial Intelligence in Agriculture. In: Bulletin UASVM Agriculture 68 (1): 284-293.

RIAL-LOVERA, K. (2018): Agricultural Robots: Drivers, Barriers and Opportunities for Adoption. In: N. TREMBLAY (Hrsg.): Proceedings of the 14th International Conference on Precision Agriculture, Montreal, 2427 June 2018. The International Society of Precision Agriculture, Monticello Illinois.

Roldán, J. J., Cerro, J. D., Garzón-Ramos, D., Garcia-Aunon, P., Garzón, M., LeÓn, J. D. und A. BARRIENTOS (2018): Robots in Agriculture: State of Art and Practical Experiences. In: A. J. R. NEVES (Hrsg.): Service Robots. IntechOpen Limited, London, doi: 10.5772/65536.

SACK, W. (2005): Eicher-Traktoren und Landmaschinen. Podszun Verlag, Brilon.

SCHImmelpfennig, D. und R. EBel (2016): Sequential Adoption and Cost Savings from Precision Agriculture. In: Journal of Agricultural and Resource Economics 41 (1): 97-115.

SCHLEICHER, S. und M. GRANDORFER (2018): Digitalisierung in der Landwirtschaft: Eine Analyse der Akzeptanzhemmnisse. In: RUCKELSHAUSEN, A., MEYer-AUrich, A., BORCHARDT, K., HOFACKER, C., LOY, J.-P., Schwerdtfeger, R., Sundermeier, H.-H., Floto, H. und B. Theuvsen (Hrsg.): Digitale 
Marktplätze und Plattformen. Lecture Notes in Informatics 278, Gesellschaft für Informatik, Bonn: 203-206.

SCHUELLER, J. K. (2006): Section 4.1 Automation and Control. In: A. MUNACK (Hrsg.): CIGR Handbook of Agricultural Engineering. The International Commission of Agricultural Engineering, Michigan: 185195, doi: 10.13031/2013.21673.

SHAMSHIRI, R. (2013): A Review of Greenhouse Climate Control and Automation Systems in Tropical Regions. In: Journal of Agricultural Science and Applications 2 (3): 175-182, doi: 10.14511/jasa.2013.020307.

Shamshiri, R., Weltzien, C., Hameed, I., Yule, J., Grift, T., BAlasundram, S., Pitonakova, L., AhMAD, D. und G. CHOWDHARY (2018): Research and development in agricultural robotics: A perspective of digital farming. In: International Journal of Agricultural and Biological Engineering 11 (4): 1-11, doi: 10.25165/j.ijabe.20181104.4278.

Thompson, N. M., Bir, C., Widmar, D. A. und J. R. Mintert (2018): Farmer Perceptions of Precision Agriculture Technology Benefits. In: Journal of Agricultural and Applied Economics 40: 1-22, doi: 10.1017/aae.2018.27.

Torrez, C., Miller, N., RAmSEY, S. und T. Griffin (2016): Factors Influencing the Adoption of Precision Agricultural Technologies by Kansas Farmers. URL: https://www.agmanager.info/sites/default/files /pdf/Precision\%20Ag\%20Technology\%20Adoption.pdf (Abrufdatum: 10.07.2018).

VOIGT, K.-I. (2019): Gabler Wirtschaftslexikon: Das Wissen der Experten. Springer Gabler, Wiesbaden.

Wegener, J. K., vON HÖrSTEN, D. und L.-M. Urso (2018): Mit Spot Farming zur nachhaltigen Intensivierung in der Pflanzenproduktion. In: Julius-Kühn-Archiv 458 (194), doi: 10.5073/jka.2018.458.002.

Wolfert, S., Ge, L., Verdouw, C. und M.-J. BogaArdT (2017): Big Data in Smart Farming - A Review. In: Agricultural Systems 153 (2017): 69-80, doi: 10.1016/j.agsy.2017.01.023.

XIANG, R., JIANG, H. und Y. YING (2014): Recognition of clustered tomatoes based on binocular stereo vision. In: Computers and Electronics in Agriculture 106 (2014): 75-90, doi: 10.1016/j.compag.2014.05.006.

Zhou, X. V., English, B. C., LARson, J., LAMBert, D. M., Roberts, R. K., BOYer, C. N., Velandia, M., FALCONER, L. L. und S. W. MARTIN (2017): Precision farming adoption trends in the Southern U.S. In: Journal of Cotton Science 21 (2): 143-155. 


\section{I.3 German Farmers' Attitudes on Adopting Autonomous Field Robots: An Empirical Survey}

Friedrich Rübcke von Veltheim und Heinke Heise

Geleisteter Eigenanteil an dem Beitrag

\begin{tabular}{|c|c|c|c|c|c|}
\hline $\begin{array}{l}\text { Literatur- } \\
\text { recherche }\end{array}$ & $\begin{array}{l}\text { Entwicklung } \\
\text { des } \\
\text { Forschungs- } \\
\text { rahmens }\end{array}$ & $\begin{array}{l}\text { Konzept und } \\
\text { Design der Stu- } \\
\text { die }\end{array}$ & $\begin{array}{l}\text { Auswahl, Aus- } \\
\text { führung und } \\
\text { Entwicklung } \\
\text { von Methoden }\end{array}$ & $\begin{array}{l}\text { Interpretation } \\
\text { der } \\
\text { Ergebnisse }\end{array}$ & $\begin{array}{l}\text { Konzept und } \\
\text { Schreiben des } \\
\text { Artikels }\end{array}$ \\
\hline$>90 \%$ & $>75 \%$ & $>75 \%$ & $90 \%$ & $>75 \%$ & $>75 \%$ \\
\hline
\end{tabular}

Dieser Beitrag ist so oder in ähnlicher Fassung veröffentlicht in der wissenschaftlichen Zeitschrift „Agriculture“ 2021 (11). 


\section{Inhalt}

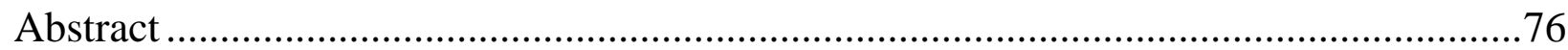

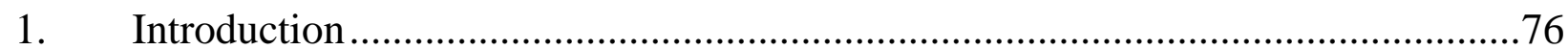

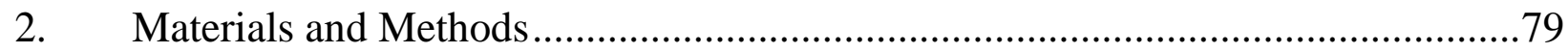

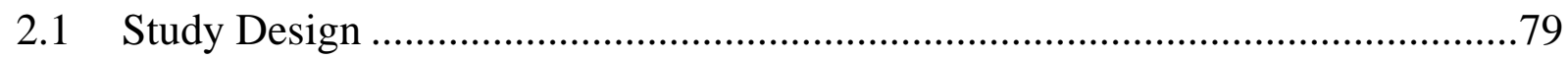

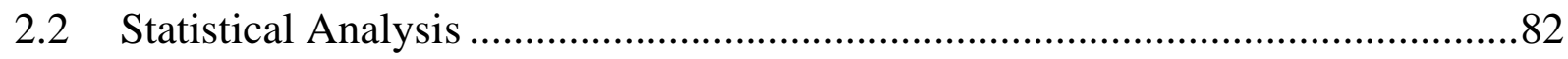

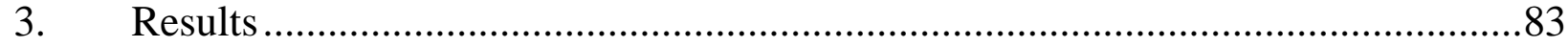

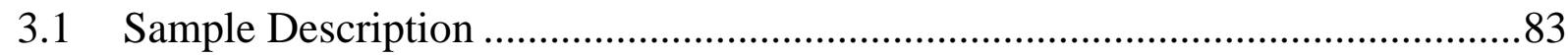

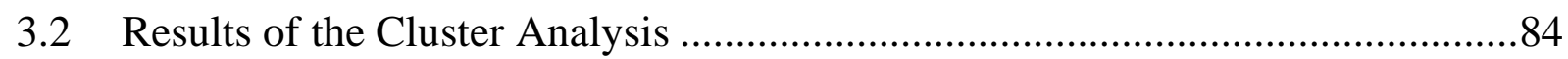

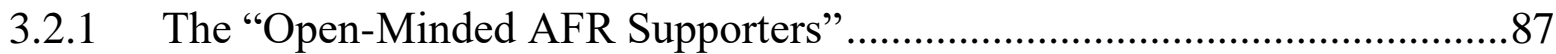

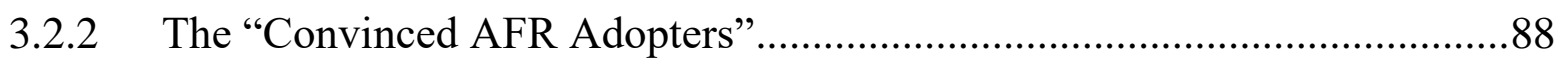

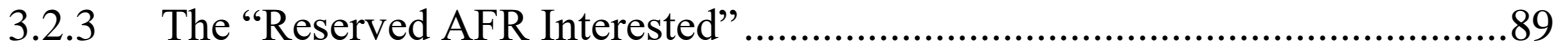

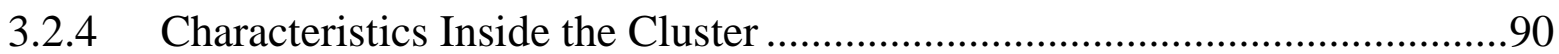

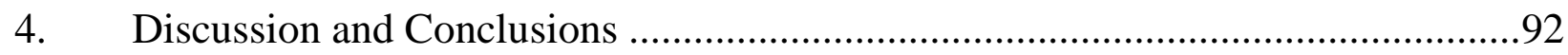

4.1 The "Open-Minded AFR Supporters"...................................................................

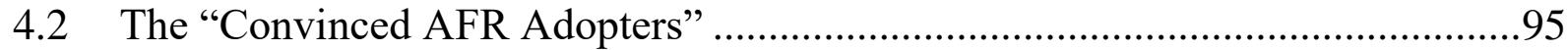

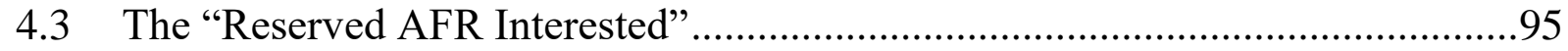

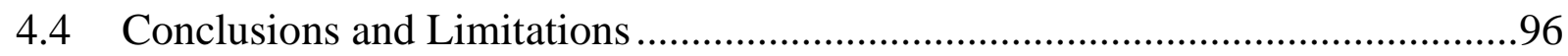

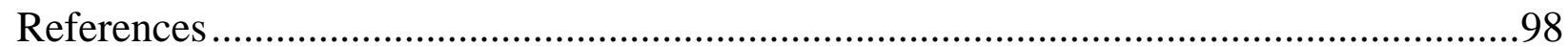

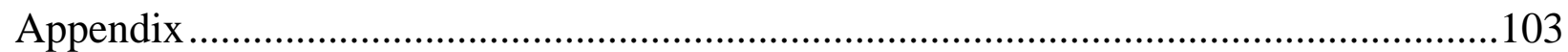




\begin{abstract}
Agricultural production methods in Europe are increasingly subject to public criticism from which many farmers suffer. This applies, among other areas, to the widespread use of pesticides. AFR, as the next stage of agricultural automation, have the potential to farm more intensively and, at the same time, in a more environmentally friendly way. However, a certain skepticism towards autonomous systems is suspected among farmers. Whether farmers adopt a technology depends largely on their uncertainty about the consequences of its use and the resulting attitude on the adoption. In order to quantify the attitude on adopting AFR in Germany and to identify possible group differences within the population, 490 German farmers were surveyed using an online questionnaire, which is based on an extended version of the Unified Theory of Acceptance and Use of Technology (UTAUT). In the subsequent cluster analysis, the statements inquiring the intention to use AFR served as cluster-forming variables. As a result, three groups ("open minded AFR supporters", "convinced AFR adopters", "reserved AFR interested") could be identified according to their response behavior. Despite existing group differences, an overall attitude in favor of AFR was observed. The results complement the existing research with a further empirical study and provide interesting starting points for further analysis, field robot manufacturers, and political decision makers.
\end{abstract}

\title{
Keywords
}

adoption; autonomous; cluster analysis; farmer, Germany; robot; technology

\section{Introduction}

The ongoing automation of mobility systems as part of the "fourth industrial revolution" (BISCHOFF, 2015) has recently gained increasing medial and, hence, societal and political relevance (RITZ, 2018; TADDICKEN et al., 2020). In addition to the car industry, this development particularly affects agriculture, which is considered a classic example of an automation process across the individual industrial revolutions (BAUERNHANSL, 2014). In contrast to industry, however, the social transformations of the $19^{\text {th }}$ century were not the consequence, but the starting point of the agricultural revolution (KROMBHOLZ, 2019). While all necessary operations (such as soil cultivation, sowing, pest control, harvesting, and threshing) were still carried out manually at that time, the increasing use of machines, such as tractors and combine harvesters, led to an ever higher degree of automation of 
agricultural production processes. Given food shortages and needs (especially during and after years of war or natural calamities) combined with the scarcity of arable land due to urban expansion, as well as its use for energy production, the associated increases in productivity were widely supported by politics and society, even though jobs were eliminated and substituted by machines (KrombHOLZ, 2019). Since the food demand of the population in industrialized countries, such as Germany, could eventually be met, process characteristics, such as the sustainability of common agricultural production methods, came to the fore of public debates and increasingly attract criticism (SPILLER et al., 2015; VOERSTE, 2008). This criticism reaches to the extent that negative effects on the reputation and the associated "license to operate" of farmers are feared (ERMANN et al., 2017).

A potential solution to the conflict between a socially demanded more sustainable food production and current agriculture, which is limited by the constraints of economic efficiency, can be found in the adoption of AFR. AFR represent a next generation of agricultural field technology, which is already highly automated in industrialized countries (RÜBCKE VON VELTHEIM et al., 2019). Operating on their own or in smart-connected swarms, AFR can perform certain operations (e.g., scouting, crop protection, or fertilization) sensor-based and more precisely than human operators. This would enable pesticide use and on-demand fertilization at an individual plant level in a more resource-efficient and environmentally friendly manner than current practices, while providing economic benefits in certain crops (LOWENBERG-DEBOER et al., 2020). Due to their relatively light weight, AFR would allow for more soil-conserving management of arable land (MINßEN et al., 2015). Besides, omitting a driver from certain farm operations would address the increasing shortage of skilled labor in agriculture and generate new jobs for maintenance, etc., of AFR in parallel. Given improved scalability of AFR, smallholder farms, which have often been deemed uneconomical, could become more profitable through the adoption of such robots leading to a rethinking process of agriculture (KING, 2017; LOWENBERGDEBOER et al., 2020). This aspect can be particularly important for organic farms, which are generally smaller than conventional ones and much more limited when it comes to the application of crop protection products. Since most AFR concepts are electrically powered, they offer farmers an opportunity to save fuel while using self-generated electricity for charging. Thus, all three pillars of sustainability (ecological, economic, and social) could be rebalanced and the previously mentioned conflicting interests appeased.

However, the introduction of unmanned vehicles into off-farm agriculture, such as AFR, is also accompanied by concerns. Although it eliminates farm machinery operators, new tasks would be added, such as monitoring and programming AFR, for which farmers may 
face a shortage of skilled labor (REDHEAD et al., 2015). DEVITT (2018) worries that AFR working out of farmers' sight may lead to a lack of trust in this technology. In addition, the successive replacement and execution of formerly manual operations by robots might result in unlearning of farming skills over time and an associated loss of social recognition for farmers (DEVITT, 2018). Regarding the adoption of smart farming technologies (SFT) in general, FLEMING et al. (2018) see the risk that mainly farmers in wealthier countries will be able to afford these technologies, which would further reduce the competitiveness of farmers in poorer countries. This is also expressed in the innovativeness-needs paradox described by ROGERS (1995), according to which, the individuals in a social system who have the greatest need for a technical innovation are the last to adopt it.

Despite all concerns, there is widespread agreement that AFR will be adopted in farming systems, the only question is when and to what extent this will happen (PICKEL, 2019; SPARROW and HOWARD, 2020). If skepticism and distrust in AFR prevail, this would hinder the development and diffusion of the technology and represent a serious adoption barrier (SPARROW and HOWARD, 2020). Following ROGERS (1995), individuals decide whether to adopt or reject an innovation whenever uncertainty about the expected consequences reaches a tolerable level. There is a wide variety of models analyzing factors influencing the user acceptance of innovations. The UTAUT model by VENKATESH et al. (2003) combines previous findings from well-documented acceptance research models and was specifically designed to predict technology acceptance. Furthermore, it has already been applied in various studies addressing issues in the agricultural context (e.g., AMBONG and PAUlinO, 2020; AMIN and LI, 2014; FAR and RAZAEI-MOGHADDAM, 2015; REDHEAD et al., 2015; RONAGHI and FOROUHARFAR, 2020; SCHUKAT et al., 2019). Thus, the UTAUT model forms the theoretical basis of our analysis. Previous studies using the UTAUT model in an agricultural context suggests that the attitudes towards the expected performance and effort, as well as the expected social influence, are relevant for the technology acceptance of farmers (e.g., CHIKOYE et al., 2018). However, previous studies on digital farming technologies in general, consisting of precision and smart farming (SCHUKAT et al., 2019), provide a diverse profile of farmers' attitudes on technology adoption depending on different parameters (DEVITT, 2018; REDHEAD et al., 2015; RIAL-LOVERA, 2018; SALIMI et al., 2020; THOMPSON et al., 2019; VECCHIO et al., 2020). This leads to the assumption that distinguishable groups also exist for farmers' attitudes toward AFR in particular.

To identify the potential target groups, this study analyzes the results of a quantitative online survey among German farmers $(n=490)$ on the expected consequences of adopting AFR. The aim is to detect different clusters and strategic groups regarding farmers' 
attitudes on adopting AFR in order to add an empirical study to the existing research. Furthermore, the results might be of interest for the strategic orientation of additional stakeholders, such as AFR manufacturers or political decision makers.

\section{Materials and Methods}

\subsection{Study Design}

The data for this study were collected using a standardized online survey on farmers' attitudes towards the adoption of AFR, in the period from July to August 2019. For this purpose, farmers $(n=490)$ in the entire federal territory of Germany were contacted via various channels, requesting them to follow a personalized hyperlink and complete the survey. Distribution channels included the email lists of the regional offices of the German Farmers' Association, published lists of German apprenticeship farms, social media websites of relevant agricultural magazines, an interview with $\mathrm{f} 3$ magazine, and private contacts within the agricultural community (network sampling approach). This approach is not uncommon in agriculture (e.g., ORSINI et al., 2020) but means that the results must be interpreted with caution due to incomplete representativeness. In order to prevent a misunderstanding of the questions, the survey was pre-tested for two weeks by experts from agri-economics research and farmers using the software Unipark (Globalpark AG). Since it was not possible to assume homogeneous knowledge of AFR among the respondents, the survey was preceded by a three-minute informational video on the functionality and possible applications of AFR presented as objectively as possible. In addition to nominal scaled questions about socioeconomic characteristics and economic practices, control questions were included to ensure the quality of responses.

The questions used were derived from a comprehensive literature analysis (e.g., DEVITT 2018; PIERPAOLI et al., 2013; REDHEAD et al., 2015; RIAL-LOVERA, 2018) and were based on the UTAUT model by VENKATESH et al. (2003) (Figure 1). Thus, different statements for the underlying key constructs performance expectancy, effort expectancy, and social influence, as well as for the moderators age, gender, and experience were formulated as closed questions. Following VENKATESH et al. (2012), the moderator voluntariness was not included in the research model of this work because farmers are free to decide whether to use AFR. Performance expectancy is defined in this paper following DAVIS (1989) as the degree to which a farmer believes that using AFR would enhance his or her job performance. To define effort expectancy, the definition of the construct perceived ease of use from DAVIS (1989) was combined with the findings of RADNER and ROTHSCHILD (1975). 
In this study, it includes variables describing the degree to which a farmer believes that the use of AFR is free of finite resources that he or she may allocate to the various farm activities for which he or she is responsible. Social influence is defined in this paper as the degree to which a farmer perceives that his or her social environment believes he or she should use AFR (VENKATESH et al., 2003).

Aside from these key constructs, existing studies on technology adoption in agriculture indicate that other factors influence farmers' adoption decisions as well (D'ANTONI et al., 2012; DevitT, 2018; HeERINK et al., 2010; Kulviwat et al., 2007; KUTTER et al., 2011; MCAllister, 1995; NeYER et al., 2012; VeNKATESH et al., 2011). Therefore, the factors trust, anxiety, and technology commitment (divided into the three facets of technological interest, technological competence beliefs, and technological control beliefs) were additionally surveyed in terms of AFR among farmers. Trust is defined in this study as the belief in the performance of AFR with personal integrity and reliability (HEERINK et al., 2010). Trust in a technology is essential for its successful diffusion and has been proven to influence adoption decisions among farmers (HEERINK et al., 2010; MACMILLAN et al., 2005; VENKATESH et al., 2011), while a lack of trust in technologies can be a cognitive barrier to adoption (DEVITT, 2018). The antonym of trust is represented by anxiety about a technology (BREHM, 1966). Anxiety is defined in this study as eliciting negative emotional reactions from AFR use (HEERINK et al., 2010). Technology Commitment provides a validated psychometric factor that addresses the use of new technologies in general (NEYER et al., 2012). The factor is composed of three facets: technological interest, technological competence beliefs, and technological control beliefs. 


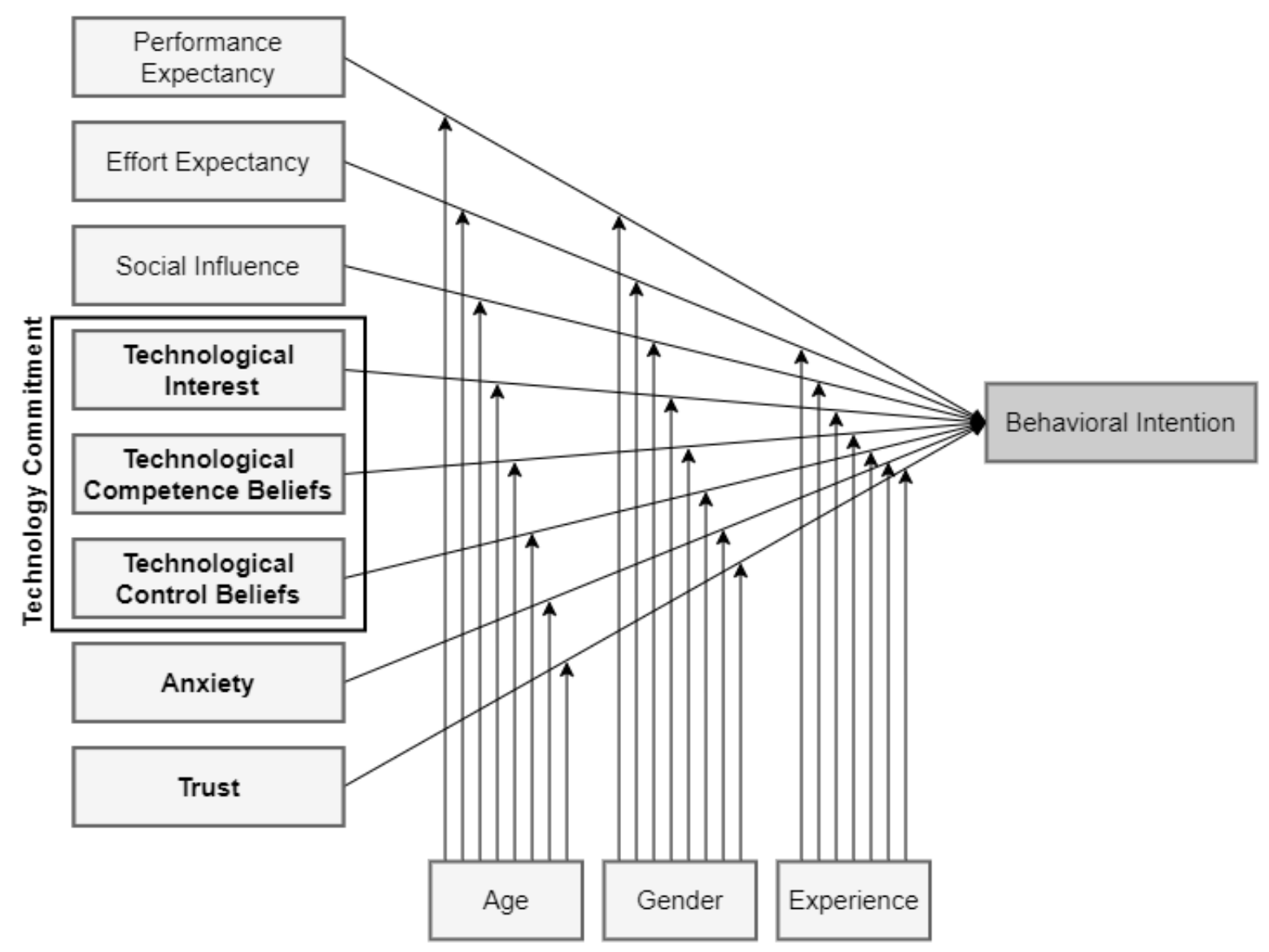

Figure 1: Modified UTAUT model in the context of AFR; bold letters = added factors

\section{Source: Authors' illustration}

To answer the survey, the farmers had to confirm or deny these statements by means of a 5-point Likert scale, where 1 = "totally disagree" and 5 = "totally agree". Further, farmers were asked to indicate their attitude towards the use of AFR on an 8-point acceptanceunacceptance scale (SAUER et al., 2005 based on HOFINGER, 2001). This scale ranges from $1=$ "I oppose the use of AFR" to $8=$ "I promote the use of AFR" and is available in the attached questionnaire. The dataset was examined for inconsistencies. For example, if the response time was far below average (average was $18 \mathrm{~min}$ ), if there was systematic response behavior, or if the control questions were answered incorrectly. After data cleaning, 490 datasets remained for further analysis. 


\subsection{Statistical Analysis}

The analysis was done using the software "IBM SPSS Statistics Subscription (2020)". First, the socio-demographic attributes of the sample and information on the farm structure of the surveyed farmers were analyzed using univariate methods. To better interpret the set of items for further analysis and to verify the constructs obtained from the literature, a hierarchical cluster analysis was performed. The four statements on intention to use AFR served as cluster-forming variables (Table 1). The goal was to form groups (clusters) that were as homogeneous as possible from the heterogeneous population. This was done by first removing outliers by applying the single-linkage method, whereby the clusters with the smallest distance (in this study: squared Euclidean distance) to each other were determined (EVERITT et al., 2011). The optimal number of clusters was then determined using Ward's method supported by the elbow criterion. In this process, individual clusters were combined with each other and checked if the variance thereby increased as little as possible (BACKHAUS et al., 2016). The result was finally compared with the dendrogram. In this way, the optimal number of three clusters could be identified.

Subsequently, the results of the hierarchical cluster analysis were compared with the results of the k-means cluster analysis to improve interpretability, and then tested in a discriminant analysis (BACKHAUS et al., 2016). To check the suitability of the sample for a discriminant analysis, the eigenvalues with the canonical correlations of the discriminant functions and their Wilks-Lambda value served as quality criteria. Eigenvalues provide information about the relative efficacy of the discriminant functions. It should be as large as possible, like the canonical correlations, which was achieved in the present study. Wilks-Lambda resulted relatively low for all three discriminant functions, meaning that the groups differed well from each other. These differences were tested for significance using the chi-square test. They all proved to be highly significant $(p \leq 0.001)$. Thus, the model was suitable for discriminant analysis. In the discriminant analysis, the correct cluster could be predicted for $85.6 \%$ of the cases, fulfilling the requirements in the literature (BACKHAUS et al., 2016). To analyze the differences between the identified clusters, mean differences were compared by univariate ANOVA table and the conservative post hoc multiple comparison test Tamhane T2. Finally, the clusters were considered according to the socio-demographic characteristics of the individual cases for possible correlations. 


\section{Results}

\subsection{Sample Description}

The sample $(n=490)$ is composed of $91.6 \%$ men and $8.4 \%$ women. This approximately represents the relative composition of the population of farmers in Germany (90.4\% male; $9.6 \%$ female) (EUROSTAT, 2020). The average age of the participants was 43 years, which was not representative for the population (53 years). The age structure of the sample was more youthful (sample: $57 \%$ younger than 45 years; national average: $38 \%$ ) (GERMAN FARMERS' ASSOCIATION, 2020). Nearly half of the participants (48\%) held a university degree, which was disproportionately high compared to the population of German farmers (12\%) (GERMAN FARMERS' ASSOCIATION, 2020). Despite the relatively low average age and higher education level, work experience was not low. For example, about $67 \%$ of respondents have worked in agriculture for more than 10 years. Moreover, $36 \%$ of the respondents originated from the northern German federal states (Bremen, Hamburg, Lower Saxony and Schleswig-Holstein) followed by $20 \%$ from the eastern states (Berlin, Brandenburg, Mecklenburg-Western Pomerania, Saxony, Saxony-Anhalt, and Thuringia), $18 \%$ from the south-western states (Baden-Württemberg, Rhineland-Palatinate, and Saarland), $16 \%$ from the western states (North Rhine-Westphalia and Hesse), and $10 \%$ from the south-eastern state of Bavaria. The German average contrasts with the sample, as most farmers are located in Bavaria (33\%) and the fewest in the eastern states (10\%) (GERMAN FARMERS' ASSOCIATION, 2020). The average farm size as well is not consistent with the German population, insofar as small farms (5 to 19 hectares) are underrepresented with $4.9 \%$ (national average: $6.6 \%$ ) and large farms (200 to 499 hectares) are overrepresented with $20.6 \%$ (national average: $16.3 \%$ ). The distribution of farm sizes in the sample almost matches the national average, with most farms (sample: $21.9 \%$; Germany: $20.4 \%$ ) in the 100 to 199 hectares class (GERMAN FARMERs' Association, 2020). Most respondents ( $86 \%$ ) have farming as their main occupation, which is only about $46 \%$ of the national average. The sample distribution by type of farming (organic or conventional) differs from the German average. In the sample, $79 \%$ of the respondents farmed conventionally (national average: $87.1 \%$ ) and $21 \%$ organically (national average: $12.9 \%$ ) (GERMAN FARMERS' ASSOCIATION, 2020). 


\subsection{Results of the Cluster Analysis}

The four statements on farmers' intention to use AFR served as cluster-forming variables for the cluster analysis performed (Table 1). This was done to divide the surveyed farmers into distinct groups based on their attitudes towards the adoption of AFR for better interpretation of the data. The results of the cluster analysis are illustrated in Table 1, showing the mean values and standard deviations of the four cluster-statements.

Table 1: Results of the clustering variables

\begin{tabular}{|c|c|c|c|c|}
\hline Statements & $\begin{array}{l}\text { CLUS A } \\
(n=257)\end{array}$ & $\begin{array}{l}\text { CLUS B } \\
(n=151)\end{array}$ & $\begin{array}{l}\text { CLUS C } \\
(n=82)\end{array}$ & $\begin{array}{c}\text { Total } \\
(n=490)\end{array}$ \\
\hline I would use AFR immediately on my farm. ${ }^{* * *}$ & $\begin{array}{c}3.46^{\mathrm{bc}} \\
(0.744)\end{array}$ & $\begin{array}{c}4.38^{\mathrm{ac}} \\
(0.500)\end{array}$ & $\begin{array}{c}1.87^{\mathrm{ab}} \\
(0.643) \\
\end{array}$ & $3.47(1.056)$ \\
\hline I plan to use AFR on my farm in the future. ${ }^{* * *}$ & $\begin{array}{l}2.48^{\mathrm{bc}} \\
(0.880)\end{array}$ & $\begin{array}{c}3.83^{\mathrm{ac}} \\
(0.781)\end{array}$ & $\begin{array}{l}1.33^{\mathrm{ab}} \\
(0.498)\end{array}$ & $2.70(1.160)$ \\
\hline $\begin{array}{l}\text { I'm sure I would spend time studying AFR during } \\
\text { the first days they are used on my farm. }\end{array}$ & $\begin{array}{l}4.43^{\mathrm{bc}} \\
(0.682)\end{array}$ & $\begin{array}{r}4.97^{\mathrm{ac}} \\
(0.161) \\
\end{array}$ & $\begin{array}{r}4.07^{\mathrm{ab}} \\
(0.813) \\
\end{array}$ & $4.54(0.688)$ \\
\hline $\begin{array}{l}\text { I would plan to learn how to properly operate AFR } \\
\text { during the first days they are used on my farm. }{ }^{* * *}\end{array}$ & $\begin{array}{l}4.53^{\mathrm{bc}} \\
(0.619)\end{array}$ & $\begin{array}{l}5.00^{\mathrm{ac}} \\
(0.000)\end{array}$ & $\begin{array}{c}4.06^{\mathrm{ab}} \\
(0.866)\end{array}$ & $4.61(0.642)$ \\
\hline
\end{tabular}

Significance level at $* \mathrm{p} \leq 0.05 ; * * \mathrm{p} \leq 0.01 ; * * * \mathrm{p} \leq 0.001$; a-c signify a significant difference to the corresponding cluster (Tamhane post-hoc multiple comparison test at significance level 0.05). Number without parentheses: mean values; numbers within parentheses: standard derivations. All statements were scored with a scale from $1=$ "totally disagree" to $5=$ "totally agree". $n=490$.

Source: Authors' calculations and illustration

Three clusters were formed (CLUS A, CLUS B, CLUS C). CLUS A as the first of the three identified clusters is the biggest one and composed of 257 farmers. This cluster is characterized by an open to slightly AFR favorable setting, but does not yet plan to adopt it ( $\mu=$ $2.48 ; \sigma=0.880$ ), which is why they are labeled the "open minded AFR supporters" in the following. Once the time is ready, they are slightly willing to immediately use AFR on their farm ( $\mu=3.46 ; \sigma=0.744)$ and they are willing to spend time studying the technology in depth $(\mu=4.43 ; \sigma=0.682)$. Even more, they are willing to learn how to operate AFR properly $(\mu=4.53 ; \sigma=0.619)$.

The second cluster (CLUS B) includes 151 farmers and is defined as a very positive tenor towards the adoption of AFR in agriculture. Therefore, the individuals in this cluster will be labeled as the "convinced AFR adopters". They would agree to an immediate use of $\operatorname{AFR}(\mu=4.38 ; \sigma=0.500)$ and plan to use them in the future as well $(\mu=3.83 ; \sigma=0.781)$. They would be eager to interact with the technology $(\mu=4.97 ; \sigma=0.161)$ and have a very strong interest in learning how to properly operate AFR $(\mu=5.00 ; \sigma=0.000)$.

CLUS C covers the responses of 82 farmers. Farmers in this cluster have the comparatively most negative view of the sample on adopting AFR in agriculture. They are therefore 
labeled as the "reserved AFR interested". Neither would they want to use AFR on their farm immediately $(\mu=1.87 ; \sigma=0.643)$, nor do they plan to do so in the future $(\mu=1.33$; $\sigma=0.498$ ). However, if this were to happen, they would want to deal the technology $(\mu=4.07 ; \sigma=0.813)$ and learn how to use it properly $(\mu=4.06 ; \sigma=0.866)$. To get a more detailed understanding of the individual clusters, they were evaluated below in terms of influencing factors on AFR adoption as cluster descriptive variables, as mentioned in section 2 (Table 2).

Table 2: Results of the cluster descriptive variables

\begin{tabular}{|c|c|c|c|c|}
\hline Statements & $\begin{array}{l}\text { CLUS A } \\
(n=257)\end{array}$ & $\begin{array}{l}\text { CLUS B } \\
(n=151) \\
\end{array}$ & $\begin{array}{l}\text { CLUS C } \\
(n=82)\end{array}$ & $\begin{array}{c}\text { Total } \\
(n=490)\end{array}$ \\
\hline \multicolumn{5}{|l|}{ Performance Expectancy } \\
\hline $\begin{array}{l}\text { AFR could lighten my workload in certain opera- } \\
\text { tions. }{ }^{* * *}\end{array}$ & $\begin{array}{l}4.49^{\mathrm{bc}} \\
(0.656)\end{array}$ & $\begin{array}{c}4.85^{\mathrm{ac}} \\
(0.362)\end{array}$ & $\begin{array}{c}3.84^{\mathrm{ab}} \\
(0.962)\end{array}$ & $4.49(0.730)$ \\
\hline AFR enables me to save work force. ${ }^{* * *}$ & $\begin{array}{l}4.00^{\mathrm{bc}} \\
(1.029)\end{array}$ & $\begin{array}{c}4.36^{\mathrm{ac}} \\
(0.933)\end{array}$ & $\begin{array}{c}3.60^{\mathrm{ab}} \\
(1.226)\end{array}$ & $4.05(1.057)$ \\
\hline With AFR I could operate more efficiently. ${ }^{* * * *}$ & $\begin{array}{c}3.96^{\mathrm{bc}} \\
(0.865)\end{array}$ & $\begin{array}{c}4.33^{\mathrm{ac}} \\
(0.846)\end{array}$ & $\begin{array}{c}2.95^{\mathrm{ab}} \\
(1.083)\end{array}$ & $3.92(1.000)$ \\
\hline $\begin{array}{l}\text { AFR would make farming more environmentally } \\
\text { friendly. }{ }^{* * *}\end{array}$ & $\begin{array}{c}3.95^{\mathrm{bc}} \\
(0.830)\end{array}$ & $\begin{array}{c}4.22^{\mathrm{ac}} \\
(0.832)\end{array}$ & $\begin{array}{c}3.00^{\mathrm{ab}} \\
(0.962)\end{array}$ & $3.88(0.943)$ \\
\hline $\begin{array}{l}\text { Through the use of AFR, additional profit can be } \\
\text { generated. }{ }^{* * *}\end{array}$ & $\begin{array}{c}3.19^{\mathrm{bc}} \\
(0.836)\end{array}$ & $\begin{array}{c}3.62^{\mathrm{ac}} \\
(0.809)\end{array}$ & $\begin{array}{c}2.72^{\mathrm{ab}} \\
(0.939)\end{array}$ & $3.24(0.905)$ \\
\hline \multicolumn{5}{|l|}{ Effort Expectancy } \\
\hline I imagine the operation of AFR to be difficult. ${ }^{* * *}$ & $\begin{array}{l}2.89^{\mathrm{bc}} \\
(0.899)\end{array}$ & $\begin{array}{l}2.52^{\mathrm{ac}} \\
(0.859)\end{array}$ & $\begin{array}{c}3.49^{\mathrm{ab}} \\
(1.009)\end{array}$ & $2.87(0.839)$ \\
\hline $\begin{array}{l}\text { Learning how to handle AFR would be difficult for } \\
\text { me. }{ }^{* * *}\end{array}$ & $\begin{array}{c}2.30^{\mathrm{bc}} \\
(0.789)\end{array}$ & $\begin{array}{c}1.96^{\mathrm{ac}} \\
(0.734)\end{array}$ & $\begin{array}{c}2.73^{\mathrm{ab}} \\
(0.903)\end{array}$ & $2.27(0.839)$ \\
\hline $\begin{array}{l}\text { A safe handling of AFR would be difficult for } \\
\text { me. }{ }^{* * *}\end{array}$ & $\begin{array}{l}2.15^{\mathrm{bc}} \\
(0.835)\end{array}$ & $\begin{array}{c}1.89^{\mathrm{ac}} \\
(0.771)\end{array}$ & $\begin{array}{c}2.51^{\mathrm{ab}} \\
(0.864)\end{array}$ & $2.13(0.850)$ \\
\hline $\begin{array}{l}\text { The maintenance/repair of AFR would be a problem } \\
\text { on my farm. }\end{array}$ & $3.10^{\mathrm{b}}(1.056)$ & $\begin{array}{c}2.66^{\mathrm{ac}} \\
(1.059)\end{array}$ & $3.28^{\mathrm{b}}(1.080)$ & $2.99(1.087)$ \\
\hline \multicolumn{5}{|l|}{ Social Influence } \\
\hline $\begin{array}{l}\text { I think that other farmers would like to see AFR } \\
\text { used on my farm. }{ }^{* * *}\end{array}$ & $\begin{array}{c}3.27^{\mathrm{bc}} \\
(0.864)\end{array}$ & $\begin{array}{c}3.57^{\mathrm{ac}} \\
(0.893)\end{array}$ & $\begin{array}{c}2.61^{\mathrm{ab}} \\
(0.926)\end{array}$ & $3.25(0.938)$ \\
\hline $\begin{array}{l}\text { I think it would make a good impression on society } \\
\text { if I used AFR. }\end{array}$ & $\begin{array}{l}3.46^{\mathrm{bc}} \\
(0.957)\end{array}$ & $\begin{array}{l}3.96^{\mathrm{ac}} \\
(0.926)\end{array}$ & $\begin{array}{c}2.60^{\mathrm{ab}} \\
(1.064)\end{array}$ & $3.47(1.061)$ \\
\hline \multicolumn{5}{|l|}{ Trust } \\
\hline I would trust AFR to make the right decisions. ${ }^{* * *}$ & $\begin{array}{c}3.42^{\mathrm{bc}} \\
(0.801)\end{array}$ & $\begin{array}{c}3.77^{\mathrm{ac}} \\
(0.818)\end{array}$ & $\begin{array}{c}2.62^{\mathrm{ab}} \\
(0.925)\end{array}$ & $3.39(0.913)$ \\
\hline $\begin{array}{l}\text { I would follow the instructions given to me by an } \\
\qquad \text { AFR. }{ }^{* * *}\end{array}$ & $\begin{array}{c}3.13^{\mathrm{bc}} \\
(0.828)\end{array}$ & $\begin{array}{l}3.42^{\mathrm{ac}} \\
(0.852)\end{array}$ & $\begin{array}{l}2.76^{\mathrm{ab}} \\
(0.976)\end{array}$ & $3.16(0.892)$ \\
\hline
\end{tabular}




\begin{tabular}{|c|c|c|c|c|}
\hline \multicolumn{5}{|l|}{ Anxiety } \\
\hline $\begin{array}{l}\text { If I were to use AFR, I would be afraid of misusing } \\
\text { it. }\end{array}$ & $2.30^{\mathrm{b}}(0.937)$ & $\begin{array}{c}1.97^{\mathrm{ac}} \\
(0.916)\end{array}$ & $2.59^{\mathrm{b}}(1.065)$ & $2.24(0.972)$ \\
\hline Using AFR, I would be afraid to damage it. ${ }^{* * *}$ & $2.13^{\mathrm{b}}(0.983)$ & $\begin{array}{l}1.76^{\mathrm{ac}} \\
(0.854)\end{array}$ & $2.23^{\mathrm{b}}(1.092)$ & $2.03(0.977)$ \\
\hline I find AFR frightening. ${ }^{* * *}$ & $1.47^{\mathrm{c}}(0.697) 1$ & $32^{\mathrm{c}}(0.659)$ & $\begin{array}{l}2.06^{\mathrm{ab}} \\
(1.093)\end{array}$ & $1.52(0.804)$ \\
\hline I find AFR intimidating. ${ }^{* * *}$ & $\begin{array}{l}1.41^{\mathrm{bc}} \\
(0.657)\end{array}$ & $\begin{array}{c}1.22^{\mathrm{ac}} \\
(0.528)\end{array}$ & $\begin{array}{l}1.87^{\mathrm{ab}} \\
(0.953)\end{array}$ & $1.43(0.712)$ \\
\hline \multicolumn{5}{|l|}{ Technological Interest } \\
\hline I am very curious about new technical innovations. & $\begin{array}{c}4.42^{\mathrm{bc}} \\
(0.726)\end{array}$ & $\begin{array}{c}4.60^{\mathrm{ac}} \\
(0.555)\end{array}$ & $\begin{array}{c}4.13^{\mathrm{ab}} \\
(0.926)\end{array}$ & $4.42(0.733)$ \\
\hline $\begin{array}{l}\text { I am always interested in using the latest technical } \\
\text { devices. }{ }^{* * * *}\end{array}$ & $\begin{array}{c}3.47^{\mathrm{bc}} \\
(0.875)\end{array}$ & $\begin{array}{c}3.89^{\text {ac }} \\
(0.858)\end{array}$ & $\begin{array}{l}3.10^{\mathrm{ab}} \\
(0.976)\end{array}$ & $3.54(0.922)$ \\
\hline $\begin{array}{l}{[\mathrm{I}] \text { would (...) use much more frequently technical }} \\
\text { products than I currently do. }\end{array}$ & $\begin{array}{c}3.67^{\mathrm{bc}} \\
(0.998)\end{array}$ & $\begin{array}{c}3.94^{\mathrm{ac}} \\
(0.876)\end{array}$ & $\begin{array}{c}3.41^{\mathrm{ab}} \\
(1.065)\end{array}$ & $3.70(0.994)$ \\
\hline $\begin{array}{l}\text { I quickly find interest in new technical develop- } \\
\text { ments. }\end{array}$ & $4.01^{\mathrm{c}}(0.859) 4$ & $19^{c}(0.862)$ & $\begin{array}{c}3.67^{\mathrm{ab}} \\
(0.982)\end{array}$ & $4.00(0.901)$ \\
\hline
\end{tabular}

\section{Technological Competence Beliefs}

Technical innovations mostly overwhelm me. ${ }^{*} \quad 2.09(0.861) 2.01^{\mathrm{c}}(0.852) 2.30^{\mathrm{b}}(0.898) 2.12(0.876)$

\begin{tabular}{cccc}
\hline $\begin{array}{c}\text { I find it difficult to deal with new technology - as a } \\
\text { rule, I simply do not know how to do that. }\end{array}$ & $1.81^{\mathrm{b}}(0.824)$ & $\begin{array}{c}1.58^{\mathrm{a}} \\
(0.6898)\end{array}$ & $1.82(0.788) 1.76(0.803)$ \\
\hline $\begin{array}{c}\text { When dealing with modern technology, I am often } \\
\text { afraid of failing. }\end{array}$ & $1.75^{\mathrm{b}}(0.802)$ & $\begin{array}{c}1.51^{\mathrm{ac}} \\
(0.765)\end{array}$ & $1.83^{\mathrm{b}}(0.767) 1.68(0.779)$ \\
\hline
\end{tabular}

I'm afraid I'll be more likely to break down technological innovations (...).** $1.59(0.708) 1.45^{\mathrm{c}}(0.709) 1.78^{\mathrm{b}}(0.832) 1.58(0.728)$

\section{Technological Control Beliefs}

It is in my hands whether I succeed in using technical innovations (...). ${ }^{*}$

$4.14^{\mathrm{b}}(0.950) 4.41^{\mathrm{a}}(0.790) 4.26(0.858) 4.24(0.894)$

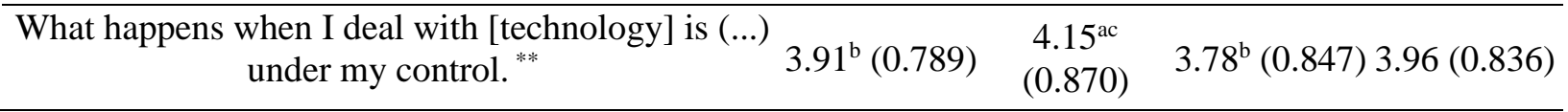

Significance level at $* \mathrm{p} \leq 0.05 ; * * \mathrm{p} \leq 0.01 ; * * * \mathrm{p} \leq 0.001$; a-c signify a significant difference to the corresponding cluster (Tamhane post-hoc multiple comparison test at significance level 0.05). Number without parentheses: mean values; numbers within parentheses: standard derivations. All statements were scored with a scale from $1=$ "totally disagree" to $5=$ "totally agree". $n=490$.

Source: Authors' calculations and illustration 


\subsubsection{The "Open-Minded AFR Supporters"}

The "open minded AFR supporters" cluster rather sees benefits in using AFR and agrees with the expected performance statements that, in doing so, their workload could be lightened ( $\mu=4.49 ; \sigma=0.604)$, work force could be saved ( $\mu=4.00 ; \sigma=1.029)$, and certain processes could be done more efficiently $(\mu=3.96 ; \sigma=0.865)$. Moreover, they are convinced that AFR enables them to operate in a more environmentally friendly way ( $\mu=3.95$; $\sigma=0.830$ ). However, the "open minded AFR supporters" are rather indifferent to the economic benefits $(\mu=3.19 ; \sigma=0.836$ ). The expected effort is slightly higher than that of the "convinced AFR adopters" and slightly lower than that of the "reserved AFR interested". They rate the operation of AFR as not being too easy $(\mu=2.89 ; \sigma=0.899)$ but see less problems in learning how to handle them properly $(\mu=2.30 ; \sigma=0.789)$. They disagree with the statement that deploying it safely would be difficult for them $(\mu=2.15 ; \sigma=0.835$ ), but vary in their opinions on whether AFR maintenance or repair would be a problem on their farm $(\mu=3.10 ; \sigma=1.056)$. Regarding the statements related to the influence of their social environment on the decision to adopt AFR by the respondents, the "open minded AFR supporters" are rather indifferent to slightly positive. The influence of society ( $\mu=3.46 ; \sigma=0.957$ ) was rated slightly higher than the influence of professional colleagues ( $\mu=3.27 ; \sigma=0.864$ ). Further, farmers from CLUS A would tend to trust the functioning of AFRs $(\mu=3.42 ; \sigma=0.801)$ rather than follow instructions from them $(\mu=3.13 ; \sigma=$ 0.828 , with an overall indifferent to slightly approving attitude on the factor trust. In contrast, no significant anxiety of the "open minded AFR supporters" regarding AFR could be measured, with the tendency of misunderstanding AFR $(\mu=2.30 ; \sigma=0.937)$ prevailing over damaging ( $\mu=2.13 ; \sigma=0.983)$ or even fearing ( $\mu=1.47 ; \sigma=0.697)$ it. In general, the results of the technology commitment factor (divided into the three facets of technological interest, technological competence beliefs, and technological control beliefs) show that farmers in CLUS A are interested in new technology, trusting in their own technical abilities, as well as the functioning of the technology, and do not fear loss of control. 


\subsubsection{The "Convinced AFR Adopters"}

The farmers of CLUS B totally agree with the statements about expected benefits from using AFR while a slight uncertainty about the economic added value of AFR could be observed $(\mu=3.62 ; \sigma=0.809)$. For instance, they see the possibility of simplifying certain work processes $(\mu=4.85 ; \sigma=0.362)$ and saving work force $(\mu=4.36 ; \sigma=0.933)$ through AFR. The expected increase in efficiency is likewise rated as positive $(\mu=4.33 ; \sigma=0.846)$. Compared to the other clusters, "convinced AFR adopters" attribute the greatest environmental added value ( $\mu=4.22 ; \sigma=0.832)$ to the use of AFR. At the same time, they are the cluster that fears the least effort due adoption of AFR. Thus, they are characterized by only a slight uncertainty about the ease of use of $\operatorname{AFR}(\mu=2.52 ; \sigma=0.859)$. In contrast, they see almost no difficulty in learning how to handle AFR $(\mu=1.96 ; \sigma=0.734)$ and in operating it safely $(\mu=1.89 ; \sigma=0.771)$. The greatest indifference occurs when assessing whether repair or maintenance of AFR would be problematic on their farm ( $\mu=2.66$; $\sigma=1.059$ ). The social influence on decision-making to adopt AFR is rated comparatively highest by CLUS B compared to the other clusters. Thus, respondents in this cluster agree with the statements that both the attitude of professional colleagues ( $\mu=3.57 ; \sigma=0.893$ ) and public opinion $(\mu=3.96 ; \sigma=0.926)$ have an influence on their decision to adopt AFR. Thereby, the attitude of society appears to be more relevant. Moreover, their trust in AFR is greater than that of CLUS A and C. Analogous to CLUS A, farmers in CLUS B would rather trust AFR to make the right decisions $(\mu=3.77 ; \sigma=0.818)$ than to follow instructions given to them by AFR $(\mu=3.42 ; \sigma=0.852)$. As a counterpart to trust, "convinced AFR adopters" expressed the fewest anxieties about this technology. Neither do they fear operating it incorrectly ( $\mu=1.97 ; \sigma=0.916)$, nor damaging it in the process $(\mu=1.76$; $\sigma=0.854)$. Least of all clusters, they find AFR frightening $(\mu=1.32 ; \sigma=0.659)$ or intimidating ( $\mu=1.22 ; \sigma=0.528$ ). Among the three identified clusters, farmers of CLUS B have the greatest technology commitment. This is expressed by a high technological interest, very low fears regarding a lack of technology competence beliefs and high technology control beliefs. 


\subsubsection{The "Reserved AFR Interested"}

In contrast to CLUS A and CLUS B, "reserved AFR interested" (CLUS C) have the lowest expectations of AFR performance. They agree that the use of AFR can both reduce the work ( $\mu=3.84 ; \sigma=0.962)$ load and save work force ( $\mu=3.60 ; \sigma=1.226)$, although the latter varies widely. However, there is an indifferent to slightly negative attitude regarding possible environmental benefits $(\mu=3.00 ; \sigma=0.962)$ and efficiency gains $(\mu=2.95$; $\sigma=1.083$ ) by using AFR. They are most critical of the possibility of generating more profit through AFR $(\mu=2.72 ; \sigma=0.939)$. In line with their more critical attitude toward possible benefits from the adoption of AFR, the "reserved AFR interested" fear the greatest effort compared with the other two clusters. Whereby this overall rather reveals an indifferent mindset. While they consider the operation of AFR to be slightly difficult ( $\mu=3.49$; $\sigma=1.009)$, they are more confident that they could learn it $(\mu=2.73 ; \sigma=0.903)$ and use it safely $(\mu=2.51 ; \sigma=0.864)$. About existing repair and maintenance possibilities on their farm, a slight skepticism could be observed ( $\mu=3.28 ; \sigma=1.080$ ). The farmers in CLUS C, compared to the "open-minded AFR supporters" and the "convinced AFR adopters", make their decision to adopt AFR least dependent on their social environment. They are not particularly interested in the opinions of their professional colleagues $(\mu=2.61 ; \sigma=0.926)$ or of society ( $\mu=2.60 ; \sigma=1.064)$. In addition, "reserved AFR interested" have little trust in how AFR works $(\mu=2.62 ; \sigma=0.925)$, which is also true for instructions that are given to them by AFR $(\mu=2.76 ; \sigma=0.976)$. Here they differ significantly from the other two clusters. Reflecting the lack of trust and compared to the "open-minded AFR supporters" and the "convinced AFR adopters", they have slightly higher levels of anxiety from using AFR, although they are dismissive of the statements on the anxiety factor overall and analogous to the other clusters. Thereby, they are most likely to fear using AFR incorrectly ( $\mu=2.59$; $\sigma=1.065$ ), which is in line with the statement of expected effort about difficulty of operation. AFRs neither really seem frightening ( $\mu=2.06 ; \sigma=1.093)$ nor intimidating ( $\mu=1.87$; $\sigma=0.953$ ) to CLUS C as well. In terms of the technology commitment factor, while their curiosity about new technology $(\mu=4.13 ; \sigma=0.926)$ coincides with that of CLUS A $(\mu=4.42 ; \sigma=0.726)$ and CLUS B $(\mu=4.60 ; \sigma=0.555)$, they are less eager to always use the latest technology (CLUS A: $\mu=3.47 ; \sigma=0.875$; CLUS B: $\mu=3.89 ; \sigma=0.858$; CLUS $\mathrm{C}: \mu=3.10 ; \sigma=0.976)$. Overall, the "reserved AFR interested" rate their technical competence beliefs slightly lower than those of the "open-minded AFR supporters" and "convinced AFR adopters", but do not fear a loss of control should they have to operate these new technologies, although this is lower than in CLUS A and B (Table 2). 


\subsubsection{Characteristics Inside the Cluster}

A more detailed description of the characteristics of those farmers within the clusters is presented in Table 3. In addition to their socio-demographic characteristics, farm statistics were also compared. No significant differences were found regarding age, gender, experience, education, vocational training, main or secondary occupation, farm type, and farm size. These characteristics are almost equally distributed across the individual clusters, although it is noticeable that the farmers in CLUS C tend to be younger and more male than those in the others. Most conventional farmers tend to be part of CLUS B. Yet, there is a significant difference regarding the farmers" willingness to take risks. Thus, the "convinced AFR adopters" are more willing to take risks $(\mu=3.56 ; \sigma=0.797)$ than the "open minded AFR supporters" $(\mu=3.35 ; \sigma=0.782)$. The "reserved AFR interested" are risk averse compared to the other two clusters, but overall, still indifferent to slightly willing to take risks $(\mu=3.13 ; \sigma=0.790)$. All clusters agree with the statement that they would be willing to make strategic changes if there was an economic benefit in doing so, with "convinced AFR adopters" agreeing fully $(\mu=4.60 ; \sigma=0.713)$ and "reserved AFR interested" agreeing only slightly $(\mu=3.51 ; \sigma=0.989)$. While the "convinced AFR adopters" would definitely want to be more environmentally friendly $(\mu=4.50 ; \sigma=0.704)$, this is true for the "open minded AFR supporters" to a not quite as strong but still clear degree $(\mu=4.23 ; \sigma=0.713)$. However, the "reserved AFR interested" are more cautious about this $(\mu=3.58 ; \sigma=1.045)$. On the 8-point acceptance-unacceptance scale presented in section 2.1, the "reserved AFR interested" rank on average between level three ("I am torn about the use of AFR") and level four ("I am indifferent to the use of AFR"), whereas the "open minded AFR supporters" find themselves in level six ("I would accept the use of AFR") and the "convinced AFR adopters" in level seven ("I approve the use of AFR"). Regarding the summarizing statement, whether the interviewed farmers consider AFR to be a good idea, a smart move or positive, highly significant differences between the individual clusters could also be identified. AFR was rated most optimistically by "convinced AFR adopters" and "open minded AFR supporters". The "reserved AFR interested" have a less positive picture than CLUS A and CLUS B, but still slightly agree with the three facets (good idea, smart move, positive) of this statement. 
Table 3: Farmers' characteristics inside the clusters

\begin{tabular}{|c|c|c|c|c|}
\hline Factor and Statements & $\begin{array}{l}\text { CLUS A } \\
(n=257)\end{array}$ & $\begin{array}{l}\text { CLUS B } \\
(n=151)\end{array}$ & $\begin{array}{l}\text { CLUS C } \\
(n=82)\end{array}$ & $\begin{array}{c}\text { Total } \\
(n=490)\end{array}$ \\
\hline Age $^{1} \varnothing$ in years ${ }^{\text {n.s. }}$ & 44 & 44 & 41 & 43 \\
\hline Gender male (female) $)^{1}$ in $\%$ n.s. & $91(9)$ & $92(8)$ & $93(7)$ & $92(8)$ \\
\hline Experience $^{1} \varnothing$ in years ${ }^{\text {n.s. }}$ & 26 & 26 & 23 & 25 \\
\hline \multicolumn{5}{|l|}{ Education $^{1}$ in $\%{ }^{\text {n.s. }}$} \\
\hline $\begin{array}{l}\text { Lower than secondary school leaving } \\
\text { certificate }\end{array}$ & 6 & 2 & 10 & 5 \\
\hline Secondary school leaving certificate & 24 & 18 & 21 & 22 \\
\hline High school diploma & 22 & 29 & 28 & 25 \\
\hline University degree & 48 & 51 & 41 & 48 \\
\hline \multicolumn{5}{|l|}{ Vocational training ${ }^{1}$ in $\%^{\text {n.s. }}$} \\
\hline Agricultural vocational training & 30 & 20 & 31 & 27 \\
\hline Agricultural university degree & 70 & 80 & 69 & 73 \\
\hline $\begin{array}{l}\text { Main occupation (secondary occupa- } \\
\text { tion) })^{1} \text { in } \% \text { n.s. }\end{array}$ & $85(15)$ & $89(11)$ & $83(17)$ & $86(14)$ \\
\hline $\begin{array}{l}\text { Farm type conventional (organic) })^{1} \\
\text { in } \%^{\text {n.s. }}\end{array}$ & $82(18)$ & $77(23)$ & $76(24)$ & $79(21)$ \\
\hline \multicolumn{5}{|l|}{ Farm size class ${ }^{1}$ in $\%$ n.s. } \\
\hline Less than 5 ha & 0 & 1 & 4 & 1 \\
\hline $5-9$ ha & 2 & 1 & 3 & 1 \\
\hline 10-19 ha & 3 & 3 & 5 & 3 \\
\hline 20-49 ha & 12 & 8 & 10 & 11 \\
\hline $50-99$ ha & 19 & 21 & 21 & 20 \\
\hline 100-199 ha & 20 & 25 & 20 & 22 \\
\hline 200-499 ha & 22 & 23 & 12 & 20 \\
\hline $500-1,000$ ha & 11 & 9 & 10 & 10 \\
\hline More than 1,000 ha & 11 & 9 & 15 & 12 \\
\hline $\begin{array}{l}\text { Are you willing to take great risks as } \\
\text { a farm manager? }{ }^{2} \mu(\sigma)^{* * *}\end{array}$ & $3.35^{\mathrm{b}}(0.782)$ & $3.56^{\mathrm{ac}}(0.797)$ & $3.13^{\mathrm{b}}(0.790)$ & $3.38(0.787)$ \\
\hline $\begin{array}{l}\text { I like to make a strategic change } \\
\text { if there is an economic benefit to doing } \\
\qquad \text { so. }^{2} \mu(\sigma)^{* * *}\end{array}$ & $4.23^{\mathrm{bc}}(0.713)$ & $4.60^{\mathrm{ac}}(0.517)$ & $3.51^{\mathrm{ab}}(0.989)$ & $\begin{array}{c}4.23 \\
(0.801)\end{array}$ \\
\hline $\begin{array}{l}\text { I would like to produce in a more envi- } \\
\text { ronmentally } \\
\text { friendly way. }{ }^{2} \mu(\sigma)^{* * * *}\end{array}$ & $4.18^{\text {bc }}(0.730)$ & $4.50^{\mathrm{ac}}(0.704)$ & $3.58^{\mathrm{ab}}(1.045)$ & $4.18(0.835)$ \\
\hline AFR acceptance level ${ }^{3} \mu(\sigma)^{* * *}$ & $6.10^{\mathrm{bc}}(1.232)$ & $6.87^{\mathrm{ac}}(0.921)$ & $4.67^{\mathrm{ab}}(1.626)$ & 6.09 (1.419) \\
\hline
\end{tabular}


In summary, I consider the use of AFR

to be ...

\begin{tabular}{ccccc}
\hline$\ldots$ a good idea $^{2} \mu(\sigma)^{* * *}$ & $4.48^{\text {bc }}(0.703)$ & $4.81^{\text {ac }}(0.412)$ & $3.40^{\text {ab }}(1.041)$ & $4.40(0.844)$ \\
\hline$\ldots$ a smart move. ${ }^{2} \mu(\sigma)^{* * * *}$ & $4.23^{\text {bc }}(0.934)$ & $4.72^{\text {ac }}(0.569)$ & $3.48^{\text {ab }}(1.080)$ & $4.26(0.953)$ \\
\hline$\ldots$ positive. $^{2} \mu(\sigma)^{* * *}$ & $4.35^{\text {bc }}(0.792)$ & $4.78^{\text {ac }}(0.462)$ & $3.39^{\mathrm{ab}}(1.015)$ & $4.33(0.875)$ \\
\hline
\end{tabular}

Significance level at $* \mathrm{p} \leq 0.05 ; * * \mathrm{p} \leq 0.01 ; * * * \mathrm{p} \leq 0.001 ; \mathrm{n} . \mathrm{s} .=$ not significant; a-c signify a significant difference to the corresponding cluster. ${ }^{1}$ Chi-square test according to Pearson. Pairwise comparison using Bonferroni correction. ${ }^{2}$ These statements were scored with a scale from $1=$ "totally disagree" to $5=$ "totally agree". Tamhane post-hoc multiple comparison test at significance level $0.05 .{ }^{3}$ These statements were scored with a scale from $1=$ "I oppose the use of AFR" to $8=$ "I promote the use of AFR". $n=490$.

Source: Authors' calculations and illustration

\section{Discussion and Conclusions}

The objective of this paper was to identify different target groups related to the attitudes of German farmers towards the adoption of AFR. For this purpose, the answers of 490 farmers could be evaluated based on an online survey.

First, it can be stated that the entire sample stands neutral to positive towards the adoption respectively the use of AFR for agriculture. These results are consistent with those of REDHEAD et al. (2015), according to whom farmers interviewed by them were enthusiastic about the opportunity to test AFR prototypes for weed control. The positive expectations of the sample are also reflected in the expected performance of AFR. The major role of expected benefits on the adoption of a new technology has been confirmed many times in the literature and is considered a basic requirement of successful innovation diffusion (MinßEN et al., 2015; PIERPAOLI et al., 2013; RIAL-LOVERA, 2018; SALIMI et al., 2020; SHOCKLEY et al., 2019; SPARROW and HOWARD, 2020). DEVITT (2018) emphasized in her study that farmers' perceptions of an overall advantage of AFR is an important adoption requirement of this technology, which seems to be given in this study. Thereby, hoping that more autonomous agricultural technology reduces the workload is in line with the results of RIAL-LOVERA (2018). In their Canadian case study, RELF-ECKSTEIN et al. (2019) confirm improved farmer health because of workload reduction and potential efficiency gains using AFR. Exploring the motives of adopting robots in agriculture, VIK et al. (2019) found that, in addition to reduced physical stress, it leads to more free time and, thus a better quality of life. LOWENBERG-DEBOER et al. (2020) and SHOCKLEY et al. (2019) found - in their analysis of the economic feasibility of AFR - that saving relatively expensive workforce for monotonous operations contributes to the economic benefits of AFR compared to existing technology. The non-perception of an economic advantage or disadvantage of AFR may be due to the partly low level of knowledge or the lack of experience 
with this technology. The missing experience might also explain the ambiguous attitude of the respondents regarding the effort expectancy. In absolute terms, however, this is still limited. Nonetheless, the tendency for farmers surveyed to be more concerned about having difficulty operating AFR is shared by REDHEAD et al. (2015). She has found that Australian farmers fear the level of complexity of AFR. For German farmers, this might become a problem as well since they also work in an industrialized country. The relevance of the feared complexity was also confirmed in the literature, including European AFR manufacturers, who see it as an important potential adoption barrier (RÜBCKE VON Veltheim and HeISE, 2020). PieRPAOLI et al. (2013) concluded in their literature review that perceived performance of new technologies only have a positive effect on the attitude towards the adoption of them if they do not lead to a significant increase in perceived effort as well. In this context, given the slight-to-large expected benefits, and little-to-almost-no feared effort across all clusters, a certain potential of future adoption and diffusion of AFR can be assumed. On the other hand, it is important to note that mainly technical effort and safety concerns were surveyed; thus, other aspects may have been neglected. At the same time, in the absence of experience with new technology, an intention-behavioral gap is often observed regarding its use (RENNER et al., 2007). The perceived neutrality, in terms of trust and social influence, can be seen as positive, as DEVITT (2018) identified a lack of trust in new technology and the loss of social cognition as important cognitive factors to the adoption of AFR. A certain social pressure on farmers in Europe, especially in Germany, results from the public debate on modern production methods in agriculture. The often emotionally charged conflict between economically driven, conventional production methods (e.g., the use of pesticides) and environmentally oriented demands from society are a burden on many farmers influencing them in their strategic decisions (MzOUGHI, 2011; ORSINI et al., 2020; SCHAAK and MUßHOFF, 2018; ZANDER et al., 2013). Farmers tend to be particularly affected by this, as they are considered important members of rural communities (RETTER et al., 2002) and are influenced in their adoption behavior by social norms (MARTÍNEZ-GARCÍA et al., 2013). At the same time, farmers are considered to be at high-risk of being partially replaced by artificial intelligence (AI) through increasing automation from 2030 onwards (FREY and OSBORNE, 2013). The absence of anxieties regarding AFR and the high level of technology commitment could be explained by the fact that farmers deal with agricultural technology on a daily basis, and this technology is constantly changing. AUSTIN et al. (1998) have already shown that farmers are more likely to use new production methods if they have a high technical interest; even more so if they want to adopt a new technology as a pioneer (Voss et al., 2008). 
Second, the partly high standard deviations in the overall sample indicate an inconsistent response behavior. It can therefore be assumed that, with regard to the adoption behavior of AFR among German farmers, there are distinguishable subgroups, analogous to the results of other studies that have investigated, for example, the attitude and investment behavior of farmers (BEER and THEUVSEN, 2019; FRANZ et al., 2012; LUHMANN et al., 2016; VON HARDENBERG and HEISE, 2018). One possible classification of such groups was introduced by ROGERS (1995). In his theory, he described the diffusion of innovations over time. Following him, innovations are initially adopted slowly by a small number of individuals, then more quickly, and by an increasing number of individuals, until a degree of saturation is reached, and they are finally adopted again more slowly by progressively decreasing numbers of individuals. He assigns a total of five groups ("innovators", "early adopters", "early majority", "late majority", and "laggards") to the respective phases of diffusion. Since this is a sample that is basically in favor of AFR, the last groups ("late majority" and "laggards") can be excluded when transferred to the identified clusters. Based on this, a cluster analysis was used to examine possible target groups in this study, whereby three distinguishable clusters (CLUS A, CLUSB, CLUS C) could be formed and compared using cluster-describing variables. These are discussed below and recommendations for further action are given.

\subsection{The "Open-Minded AFR Supporters"}

CLUS A ("open-minded AFR supporters"), which has a slightly positive stance towards the immediate use of AFR, but is not currently planning to do so, reveals neutral expectations regarding the possibility of generating additional profit through AFR within the basically positive performance expectancy, similar to the overall sample. To encourage this potential target group (which represents the largest share of the sample) to adopt AFR, its economic benefits over existing agricultural technology should be communicated to them. This is also applicable as CLUS A farmers would be willing to make strategic changes, despite low-risk appetite, should economically benefits result. For example, experience reports from test farms or studies that prove these benefits (e.g., LOWENBERG-DEBOER et al., 2020) could be presented in practical workshops. Similarly, an attempt would have to be made to target the other uncertainties, such as the feared complexity of AFR operations or certainty regarding their repair and maintenance options. First, this can be done through practical field demonstrations. Second, it places the demand on AFR manufacturers to design the technology in such a way that it is compatible with existing agricultural technology or the prevailing circumstances on the farm. It is not the farmer who should adapt to the technology, but the technology to the farmer (VIK et al., 2019). If the effort required to 
operate AFR (e.g., to calibrate the sensors) is too high, this can have a negative impact on farmers' attitudes towards the adoption of the technology (DEVITT, 2018). Besides, efforts should be made to emphasize the benefits of AFR for society (such as less emitted noise near populated areas or ecological benefits, such as less pesticide use and less soil compaction), since the "open-minded AFR supporters" on the one hand can be influenced by society's view of AFR, and on the other hand, want to farm in a more environmentally friendly way.

\subsection{The "Convinced AFR Adopters"}

CLUS B ("convinced AFR adopters"), the second largest cluster, would use AFR immediately and is planning to do so in the future. Due to the high affinity of the "convinced AFR adopters" towards the adoption of AFR, this target group should be particularly courted, as they are likely to be the first to adopt AFR. If the positive expectations of these pioneer farmers are confirmed as a result of AFR use, this could convince more farmers of its viability as a promising technology. Since farmers in this cluster are more willing to take risks and more technophile than farmers in the other two clusters, AFR manufacturers could establish test farms among the "convinced AFR adopters". This could, on the one hand, address existing uncertainties regarding compatibility with existing agricultural technology and additional profit from the use of AFR and, on the other hand, generate technical input from farmers on the practical feasibility of AFR without losing them as potential customers. Besides the strong economic influence in their strategic farm orientation, farmers of CLUS B are influenced in their adoption decision by the ecological added value of AFR, and especially by the society's mood, which is why this should be particularly addressed. The "convinced AFR adopters" "trust in the functioning of AFR can also be considered positive for its fast adoption, as it is a lack of reliability in new technologies such as AFR that represents an important adoption barrier (DEVITT, 2018; REDHEAD et al., 2015).

\subsection{The "Reserved AFR Interested"}

In order to attract the smallest and most skeptical of the identified clusters (CLUS C; "reserved AFR interested") as a target group to adopt AFR, greater efforts have to be made, as they would neither use AFR immediately nor plan to do so in the future. The non-expected efficiency gains and increased environmental performance from the use of AFR can be refuted with various studies. However, it is not certain that this alone would be enough, as farmers prefer to avoid risk rather than increase efficiency (TEY and BRINDAL, 2012). The slight risk aversion of CLUS C farmers might be addressed in several ways. For 
instance, insurance offers and personal customer support on the part of the manufacturer or risk mitigation for farmers trialing new technologies on the part of policymakers could be an option (DEVITT, 2018). Farmers are more inclined to feel that technologies are good or bad based on their own experiences or the experiences of others. Since "reserved AFR interested" are unlikely to be influenced in their decision-making by their social environment, targeted field trials would also be an option for increasing acceptance among this target group. At the same time, this could reduce the anxiety of being overwhelmed by the operation of AFR, provided that AFR manufacturers implement a simple user interface design (DEVITT, 2018). Therefore, as in Australia, CLUS C could be more strongly involved in product development and thus, further promote existing potentials, while reducing possible uncertainties about this technology (REDHEAD et al., 2015). Ignorance of operational requirements or compatibility of AFR with existing equipment could be alleviated through focused training or workshops, analogous to CLUS A and CLUS B.

\subsection{Conclusions and Limitations}

If the identified clusters of this study are finally transferred to the groups of the theory of diffusion of innovations by ROGERS (1995), and compared with their classification on the surveyed 8-point acceptance-unacceptance scale, the "convinced AFR adopters" who assign themselves to level seven ("I would approve the use of AFR") correspond most closely to the "innovators", the "open-minded AFR supporters" who assign themselves to level six ("I would accept the use of AFR") correspond to the "early adopters", the "reserved AFR interested" who assign themselves between level three ("I am torn about the use of AFR") and four ("I am indifferent to the use of AFR") to the "early or late majority", as CLUS C would be the last to use AFR immediately, despite the perceived benefits at a manageable cost (ROGERS, 1995). In this context, it would probably be purposeful to address each group in its corresponding phase, starting with the large potential of "convinced AFR adopters". On the other hand, all clusters are at an early stage of opinion formation about AFR, since only a few farmers are likely to be well informed about such a new technology. This would be supported by the indifferent attitude of the "reserved AFR interested" regarding the factor performance expectancy, which could indirectly turn positive by a better communication of, e.g., the ecological advantages of AFR in society.

Nevertheless, the results must be viewed with caution given its limitations. Since the hyperlink to the survey was sent by mail, it can be assumed that farmers interested in AFR or AFR opponents tended to respond to the call (self-selection bias) and, thus, a substantial part of the population remained unnoticed (JACOBS et al., 2009). Furthermore, respondents 
in such surveys tend not to express their true opinions, but succumb to the phenomenon of social desirability, or show a tendency towards the middle (in this case 3 out of 5) in their response behavior (BORTZ and DÖRING, 2006). Compared to the population of farmers in Germany, the age and farm size structure of the sample studied is younger and larger, which could result in a more technophile setting among the respondents, although this may not be misguided against the background of a future technology, such as AFR. On the other hand, given the economic benefits of AFR especially for smaller farms, the larger farm structure of the sample could lead to an underestimation of the actual attitudes towards the adoption of AFR. In addition, relatively high standard deviations appear sporadically in the results. This is an indication that the clusters formed are partly heterogeneous and combine farmers who would possibly form a separate cluster. Further, it must be noted that AFR adoption in general was asked about. The attitude of the farmers could be more positive or more negative depending on the specific AFR type.

Despite these limitations, this study provides interesting results on the attitudes of German farmers towards the adoption of AFR. Especially the knowledge about the characteristics of different target groups is essential to address them systematically and, thus, promoting the diffusion of this new technology. The results are likely to be of interest to AFR manufacturers, which were largely made up of startups, as successful establishment, and diffusion of AFR is probably vital to their future success. Disregarding any claim to representativeness of the sample, there seems to be a lot of interest in this new technology that AFR manufacturers can build on, for example, by better promoting the benefits of AFR to society, as they in turn influence policy and, thus, not only directly, but also indirectly, farmers in their farming activities. In addition, field demonstrations or targeted training opportunities could reduce the expected effort among farmers and increase their knowledge of the benefits of the new technology. Since many AFRs are still in the prototype phase, it would also be conceivable to involve farmers in the AFR production process (REDHEAD et al., 2015). This would more quickly reduce uncertainty about farmers' consequences to a tolerable level, which, according to ROGERS (1995), would lead to an eventual adoption decision. Since the use of AFR would reduce production costs, especially on small to medium-sized farms, and these would henceforth be less dependent on government subsidies, politicians might also have a certain interest in promoting AFR (LOWENBERG-DEBOER et al., 2020). They have various funding instruments that could act either as a supply-side promotion, in the sense of a technology push, or as a demand-side promotion, in the sense of a market pull (GERYBADZE, 2015). 
Finally, the results motivate further studies. It would be interesting, for example, to survey the perspective of other stakeholders, such as agricultural machinery manufacturers or society, on AFR and to compare it with the farmers' one. After all, a new technology increasingly stands and falls with its public backing (i.e., genetically modified plants) (FERRETTI and PAVONE, 2009). Methodologically, a more in-depth analysis of influencing directions of the factors formed would be plausible to better understand the cause-effect correlations. For example, choice-based experiments could be used to explore the conditions under which farmers would be willing to adopt this new technology or to determine the willingness to pay for AFR. In addition, it would be interesting to determine the de facto sustainability of AFR with the help of a life cycle assessment.

\section{Acknowledgement}

We acknowledge support by the Open Access Publication Funds of the Göttingen University.

\section{References}

Ambong, R. M. A. and M. A. Paulino (2020): Analyzing Rice Farmers' Intention to Adopt Modern Rice Technologies Using Technology Acceptance Model (TAM). In: Asian Research Journal of Agriculture 13 (1): 21-30, doi: 10.9734/arja/2020/v13i130094.

AMIN, K. and J. LI (2014): Applying farmer technology acceptance model to understand farmer's behavior intention to use ICT based microfinance platform: A comparative analysis between Bangladesh and China. Wuhan International Conference on e-Business (WHICEB) Proceedings at the AIS Electronic Library (AISeL), Wuhan, China.

Austin, E. J., Willock, J., Deary, I. J., Gibson, G. J., Dent, J. B., Edwards-Jones, G., Morgan, O., GRIEVE, R. and A. SUTHERLAND (1998): Empirical models of farmer behaviour using psychological, social and economic variables. Part I: linear modelling. In: Agricultural Systems 58 (2): 203-224, doi: 10.1016/S0308-521X(98)00066-3.

Backhaus, K., Erichson, B., Weiber, R. and W. Plinke (2016): Multivariate Analysemethoden: Eine anwendungsorientierte Einführung. Springer, Berlin/Heidelberg, doi: 10.1007/978-3-662-46076-4.

BAuernhansL, T. (2014): Die Vierte Industrielle Revolution - Der Weg in ein wertschaffendes Produktionsparadigma. In: BAuernhansl, T., TEN Hompel, M. and B. Vogel-Heuse (Eds.): Industrie 4.0 in Produktion, Automatisierung und Logistik. Springer Vieweg, Wiesbaden: 5-35, doi: 10.1007/978-3-658-04682-8_1.

BEER, L. and L. THEUvSEN (2019): Conventional German farmers' attitudes towards agricultural wood and their willingness to plant an alley cropping system as an ecological focus area: A cluster analysis. In: Biomass and Bioenergy 125 (2019): 63-69, doi: 10.1016/j.biombioe.2019.04.008.

Bischoff, J. (2015): Industrie 4.0 im Mittelstand. Erschließen der Potenziale der Anwendung von Industrie 4.0 im Mittelstand. Agriplan GmbH, Mühlheim an der Ruhr.

BORTZ, J. and N. DÖRING (2006): Forschungsmethoden und Evaluation für Human- und Sozialwissenschaftler. Springer, Berlin/Heidelberg, doi: 10.1007/978-3-540-33306-7.

BREHM, J. W. (1966): A theory of psychological reactance. Academic Press, New York. 
ChikoYe, D. M., GUPTA, N. K. and K. R. KANDADI (2018): Application of UTAUT in understanding the adoption of technologies for reducing post harvest maize in Zambia. In: International Journal of Agriculture and Environmental Research 4 (3): 610-636.

D'ANTONi, J. M., MishrA, A. K. and H. JoO (2012): Farmers' perception of precision technology: The case of autosteer adoption by cotton farmers. In: Computers and Electronics in Agriculture 87: 121-128, doi: 10.1016/j.compag.2012.05.017.

Davis, F. D. (1989): Perceived Usefulness, Perceived Ease of Use, and User Acceptance of Information Technology. In: MIS Quarterly 13 (3): 319-340, doi: 10.2307/249008.

DevitT, S. K. (2018): Cognitive Factors that Affect the Adoption of Autonomous Agriculture. In: Farm Policy Journal 15 (2): 49-60.

ERMANN, M., CHRISTOPH-SCHUlZ, I. and A. SPILLER (2017): Under pressure - Wie nehmen Landwirtinnen und Landwirte in Deutschland den Druck externer Stakeholder wahr? In: Yearbook of the Austrian Society of Agricultural Economics 26: 85-94, doi: 10.24989/OEGA.JB.26.10.

Eurostat (2020): Agriculture, forestry and fishery statistics; Publications Office of the European Union, Luxembourg City.

EveritT, B. S., LANDAU, S., LEeSE, M. and D. STAHL (2011): Cluster Analysis. John Wiley \& Sons, New York City.

FAR, S. T. and K. REZAEI-MogHAdDAM (2015): Determinants of Iranian Agricultural Consultants' Intentions toward Precision Agriculture: Integrating Innovativeness to the Technology Acceptance Model. In: Journal of the Saudi Society of Agricultural Sciences 16 (3): 280-286, doi: 10.1016/j.jssas.2015.09.003.

FERRETTI, M. P. and V. PAVONE (2009): What do civil society organisations expect from participation in science? Lessons from Germany and Spain on the issue of GMOs. In: Science \& Public Policy 36 (4): 287-299, doi: 10.3152/030234209X436527.

Fleming, A., JAKKU, E., LIM-CAMACHO, L., TAYLOR, B. and P. THORBURN (2018): Is big data for big farming or for everyone? Perceptions in the Australian grains industry. In: Agronomy for Sustainable Development 38, 24 (2018), doi: 10.1007/s13593-018-0501-y.

FrANZ, A., DeIMEL, I. and A. SPILLER (2012): Concerns about animal welfare: A cluster analysis of German pig farmers. In: British Food Journal 114 (10): 1445-1462, doi: 10.1108/00070701211263019.

Frey, C. B. and M. A. Osborne (2013): The future of employment: How susceptible are jobs to computerization. Oxford Martin School, University of Oxford, Oxford. URL: https://www.oxfordmartin.ox.ac.uk/downloads/academic/future-of-employment.pdf (accessed on 30 February 2021).

GERMAN FARMERS' AsSOCIATION (2020): Situationsbericht 2020/21. Trends und Fakten zur Landwirtschaft. DBV, Berlin.

GERYBADZE, A. (2015): Instrumente der Innovationspolitik. Auf dem Weg zu einer neuen Industriepolitik? In: WSI-Mitteilungen 68 (7): 516-525, doi: 10.5771/0342-300X-2015-7-516.

HeErinK, M., Krose, B., Evers, V. and B. Wielinga (2010): Assessing Acceptance of Assistive Social Agent Technology by Older Adults: the Almere Model. In: International Journal of Social Robotics 2 (4): 361375, doi: 10.1007/s12369-010-0068-5.

Hofinger, G. (2001): Denken über Umwelt und Natur. Beltz PVU, Weinheim.

Jacobs, B., Hartog, J. and W. Vijverberg (2009): Self-selection Bias in Estimated Wage Premiums for Earnings Risk. In: Empirical Economics 37 (2): 271-286, doi: 10.1007/s00181-008-0231-0.

KING, A. (2017): Technology: The Future of Agriculture. In: Nature 544 (7651): 21-23, doi: 10.1038/544S21a.

KROMBHOLZ, K. (2019): Gedanken zur Vorgeschichte von Landwirtschaft 4.0. In: L. FreRICHS (Ed.): Yearbook of agricultural engineering 2018. Institute for mobile machines and commercial vehicles, Brunswick: 1-17. 
Kulviwat, S., Bruner II, G. C., Kumar, A., Nasco, S. A. and T. Clark (2007): Toward a unified theory of consumer acceptance technology. In: Psychology \& Marketing 24 (12): 1059-1084, doi: 10.1002/mar.20196.

KuTter, T., TIEMANN, S., SiEBERT, R. and S. FOUNTAS (2011): The role of communication and co-operation in the adoption of precision farming. In: Precision Agriculture 12 (2011): 2-17, doi: 10.1007/s11119009-9150-0.

Lowenberg-DeBoer, J., HuAng, I. Y., Grigoriadis, V. and E. BlackMORE (2020): Economics of robots and automation in field crop production. In: Precision Agriculture 21 (2020): 278-299, doi: 10.1007/s11119-019-09667-5.

LuHMANN, H., SCHAPER, C. and L. THEUVSEN (2016): Future-oriented dairy farmers' willingness to participate in a sustainability standard: Evidence from an empirical study in Germany. In: Journal on Food System Dynamics 7 (3): 289-301, doi: 10.18461/ijfsd.v7i3.735.

MacMillan, K., Money, K., Downing, S. and C. Hillenbrand (2005): Reputation in Relationships: Measuring Experiences, Emotions and Behaviors. In: Corporate Reputation Review 8 (2005): 214-232, doi: $10.1057 /$ palgrave.crr.1540251.

MARTÍNEZ-GARCÍA, C. G., DOWARD, P. and T. REHMAN (2013): Factors influencing adoption of improved grassland management by small-scale dairy farmers in central Mexico and the implications for future research on smallholder adoption in developing countries. In: Livestock Science 152 (2-3): 228-238, doi: 10.1016/j.livsci.2012.10.007.

MCAllister, D.J. (1995): Affect- and Cognition-Based Trust as Foundations for Interpersonal Cooperation in Organizations. In: The Academy of Management Journal 38 (1): 24-59, doi: 10.2307/256727.

MinßEN, T.-F., URSO, L.-M., GAUS, C.-C. and L. FreriCHS (2015): Mit autonomen Landmaschinen zu neuen Pflanzenbausystemen. In: ATZoffhighway 8 (2015): 6-11, doi: 10.1007/s35746-015-0028-y.

Mzoughi, N. (2011): Farmers adoption of integrated crop protection and organic farming: Do moral and social concerns matter? In: Ecological Economics $70 \quad$ (8): 1536-1545, doi: 10.1016/j.ecolecon.2011.03.016.

Neyer, F. J., Felber, J. and C. Gebhardt (2012): Entwicklung und Validierung einer Kurzskala zur Erfassung von Technikbereitschaft. In: Diagnostica 58 (2): 87-99, doi: 10.1026/0012-1924/a000067.

Orsini, S., Costanzo, A., Solfaneli, F., Zanoli, R., Padel, S., Messmer, M. M., Winter, E. and F. SCHAEFER (2020): Factors Affecting the Use of Organic Seed by Organic Farmers in Europe. In: Sustainability 2020 (12): 8540, doi: 10.3390/su12208540.

PICKEL, P. (2019): RoundTable: Agricultural robotics storms global market. Presentation on the International Forum of Agricultural Robotics (FIRA) 2019, Toulouse.

Pierpaoli, E., Carli, G., PignatTi, E. and M. Canavari (2013): Drivers of Precision Agriculture Technologies Adoption: A Literature Review. In: Procedia Technology 8 (2013): 61-69, doi: 10.1016/j.protcy.2013.11.010.

RADNER, R. and M. ROTHSCHILD (1975): On the allocation of effort. In: Journal of Economic Theory 10 (3): 358-376, doi: 10.1016/0022-0531(75)90006-X.

Redhead, F., SNOW, S., Vyas, D., BAwden, O., Russell, R., Perez, T. and M. Brereton (2015): Bringing the Farmer Perspective to Agricultural Robots. In: BEGole, B., KIM, J., INKPEN, K. and W. WOO (Eds.): Proceedings of the 33rd Annual ACM Conference Extended Abstracts on Human Factors in Computing Systems. Association for Computing Machinery, New York: 1067-1072, doi: 10.1145/2702613.2732894.

Relf-Eckstein, J. E., Ballantyne, A. T. and P. W. B. Phillips (2019): Farming Reimagined: A case study of autonomous farm equipment and creating an innovation opportunity space for broadacre smart farming. In: NJAS - Wageningen Journal of Life Science 90-91: 100307, doi: 10.1016/j.njas.2019.100307.

RENNER, B., SPIVAK, Y., KWON, S. and R. SCHWARZER (2007): Does age make a difference? Predicting physical activity of South Koreans. In: Psychology and Aging 22 (3): 482-493, doi: 10.1037/0882-7974.22.3.482. 
RETTER, C., STAHR, K. and H. BOLAND (2002): Zur Rolle von Landwirten in dörflichen Kommunikationsnetzwerken. In: Berichte über Landwirtschaft 80 (3): 446-467.

Rial-LoverA, K. (2018): Agricultural Robots: Drivers, Barriers and Opportunities for Adoption. In: N. TRemblay (Ed.): Proceedings of the 14th International Conference on Precision Agriculture, Montreal, 24-27 June 2018. The International Society of Precision Agriculture, Monticello Illinois.

RITZ, J. (2018): Autonome Fahrzeuge. In: J. RITZ (Ed.): Mobilitätswende - autonome Autos erobern unsere Straßen. Springer Fachmedien Wiesbaden, Wiesbaden: 27-66.

Rogers, E. M. (1995): Diffusion of Innovations. Free Press, New York.

Ronaghi, M. H. and A. Forouharfar (2020): A contextualized study of the usage of the Internet of things (IoTs) in smart farming in a typical Middle Eastern country within the context of Unified Theory of Acceptance and Use of Technology model (UTAUT). In: Technology in Society 63 (2020): 101415, doi: 10.1016/j.techsoc.2020.101415.

RÜBCKe VON Veltheim, F., Theuvsen, L. and H. Heise (2019): Akzeptanz autonomer Feldroboter im Ackerbaueinsatz: Status quo und Forschungsbedarf. In: Berichte über Landwirtschaft 97 (3): 1-19, doi: 10.12767/buel.v97i3.248.

RÜbcke von Veltheim, F. and H. Heise (2020): The AgTech Startup Perspective to Farmers Ex Ante Acceptance Process of Autonomous Field Robots. In: Sustainability 12 (2020): 10570, doi: $10.3390 /$ su122410570.

SAlimi, M., POURDARBAni, R. and B. A. NOURI (2020): Factors Affecting the Adoption of Agricultural Automation Using Davis's Acceptance Model (Case Study: Ardabil). In: Acta Technologica Agriculturae 23 (1): 30-39, doi: 10.2478/ata-2020-0006.

SAUER, A., LuZ, F., SUdA, M. and U. WeILAND (2005): Steigerung der Akzeptanz von FFH-Gebieten. In: BfNSkripten 144: 1-200.

SCHAAK, H. and O. MUßHOFF (2018): Understanding the adoption of grazing practices in German dairy farming. In: Agricultural Systems 165 (2018): 230-239, doi: 10.1016/j.agsy.2018.06.015.

SCHUKAT, S., THEUvSEN, L. and H. HeISE (2019): IT in der Landwirtschaft: mit einheitlichen Definitionen zu einheitlichem Verständnis. In: MEYER-AUriCH, A., GANDOrfer, M., BARTA, N., GronAUER, A., KANTELHARDT, J. and H. FlOTO (Eds.): Digitalisierung in kleinstrukturierten Regionen- ein Widerspruch in sich? Gesellschaft für Informatik e.V., Bonn: 211-216.

SHOCKLEY, J. M., DiLlON, C. R. and S. A. SHEARER (2019): An economic feasibility assessment of autonomous field machinery in grain crop production. In: Precision Agriculture 20 (2019): 1068-1085, doi: 10.1007/s11119-019-09638-w.

SPARROW, R. and M. HOWARD (2020): Robots in agriculture: prospects, impacts, ethics, and policy. In: Precision Agriculture 22 (2021): 818-833, doi: 10.1007/s11119-020-09757-9.

Spiller, A., Gauly, M., Balmann, A., Bauhus, J., Birner, R., Bockelmann, W., Christen, O., Entemann, S., Grethe, H., Knierim, U., Latacz-Lohmann, U., Martinez, J., Nieberg, H., QAim, M., TAube, F., Tenhagen, B.-A. and P. Weingarten (2015): Wege zu einer gesellschaftlich akzeptierten Nutztierhaltung. In: Berichte über Landwirtschaft Special issue 221: 1-171, doi: 10.12767/buel.v0i221.82.

Taddicken, M., Reif, A., Brandhorst, J., Schuster, J., Diestelhorst, M. and L. Hauk (2020): Wirtschaftlicher Nutzen statt gesellschaftlicher Debatte? Eine quantitative Framing- Analyse der Medienberichterstattung zum autonomen Fahren. In: M\&K Medien \& Kommunikationswissenschaft 68 (4): 406-427, doi: 10.5771/1615-634X-2020-4-406.

TEY, Y. S. and M. BRINDAL (2012): Factors influencing the adoption of precision agricultural technologies: a review for policy implications. In: Precision Agriculture 13 (6): 713-730, doi: 10.1007/s11119-012-92736.

Thompson, N. M., Bir, C., Widmar, D. A. and J. R. Mintert (2019): Farmer Perceptions of Precision Agriculture Technology Benefits. In: Journal of Agricultural and Applied Economics 51 (1): 142-163, doi: 10.1017/aae.2018.27. 
Vecchio, Y., De Rosa, M., Adinolfi, F., Bartoli, L. and M. MAsi (2020): Adoption of precision farming tools: A context-related analysis. In: Land Use Policy 94 (2020): 104481, doi: 10.1016/j.landusepol.2020.104481.

Venkatesh, V., Morris, M. G., Davis, G. B. and F. D. Davis (2003): User Acceptance of Information Technology: Toward a Unified View. In: MIS Quarterly 27 (3): 425-478, doi: 10.2307/30036540.

Venkatesh, V., Thong, J. Y. L., Chan, F. K. Y., Hu, P. J. H. and S. A. Brown (2011): Extending the twostage information systems continuance model - Incorporating UTAUT predictors and the role of context. In: Information Systems Journal 21(6): 527-555, doi: 10.1111/j.1365-2575.2011.00373.x.

Venkatesh, V., Thong, J. Y. L. and X. XU (2012): Consumer Acceptance and Use of Information Technology: Extending the Unified Theory of Acceptance and Use of Technology. In: MIS Quarterly 36 (1): 157-178, doi: 10.2307/41410412.

Vik, J., Stræte, E. P., Hansen, B. G. and T. NÆrLand (2019): The political robot - The structural consequences of automated milking systems (AMS) in Norway. In: NJAS - Wageningen Journal of Life Sciences 90-91 (2019): 100305, doi: 10.1016/j.njas.2019.100305.

Voerste, A. (2008): Lebensmittelsicherheit und Wettbewerb in der Distribution. Josef Eul Verlag, Siegburg.

VON HARDENBERG, L. and H. HEISE (2018): German pig farmers' attitude towards animal welfare programs and their willingness to participate in these programs: An empirical study. In: Journal on Food System Dynamics 9 (3): 289-301, doi: 10.18461/ijfsd.v9i3.937.

Voss, J., Schaper, C., SPILler, A. and L. Theuvsen (2008): Innovationsverhalten in der deutschen Landwirtschaft - Empirische Ergebnisse am Beispiel der Biogasproduktion. Paper presented at the 48th Annual Conference, Bonn, Germany, 24-26 September 2008. German Association of Agricultural Economists (GEWISOLA), Brunswick, doi: 10.22004/ag.econ.52666.

Zander, K., Isermeyer, F., BÜrgelt, D., Christoph-Schulz, I. B., SAlamon, P. and D. Weible (2013): Erwartungen der Gesellschaft an die Landwirtschaft. Stiftung Westfälische Landwirtschaft, Münster. 


\section{Appendix}

\section{Questionnaire}

\section{Socio-demographic characteristics}

How old are you?

Please indicate as a number (e.g., 54).

- Age: years old.

Please mark your gender here.

- Male (1)

- Female (2)

How many years of experience do you have working in agriculture?

Please indicate as a number (e.g., 5).

- Experience: years.

Which educational level did you graduate from?

Please mark your highest degree with a cross.

- No school-leaving certificate

- Still in school-based education

- Secondary or elementary school leaving certificate

- Graduation from polytechnic high school

- Secondary school leaving certificate

- (Technical) high school diploma

- (Specialized) university degree

What vocational training have you completed?

Please mark your highest degree with a cross.

- No vocational qualification

- Agricultural training

- Agricultural master

- 1-year technical college

- 2-year technical college

- Bachelor's degree

- Master's degree/Study Diploma

- Doctorate

\section{Questions about your farm}

In which region is your farm located?

Please tick only one box.

- North (Bremen, Hamburg, Lower Saxony, and Schleswig-Holstein)

- South-West (Baden-Württemberg, Rhineland-Palatinate, and Saarland) 
- South-East (Bavaria)

- West (Hesse and North Rhine-Westphalia)

- East (Berlin, Brandenburg, Mecklenburg-Western Pomerania, Saxony, Saxony-Anhalt, and Thuringia)

What type of business do you run on your farm?

Please tick only one box.

- Main occupation (1)

- Secondary occupation (2)

How is your farm managed?

Please tick only one box.

- Conventional (1)

- Organic (2)

Please mark with a cross approximately how many hectares of agricultural land are cultivated on your farm.

Please tick only one box.

- less than 5 ha (1)

- 5-9 ha (2)

- $\quad 10-19$ ha (3)

- $20-49$ ha (4)

- 50-99 ha (5)

- 100-199 ha (6)

- 200-499 ha (7)

- 500-1000 ha (8)

- more than 1000 ha (9)

What percentage of your farmland is leased?

To do this, please drag the slider (click and hold) to the side until the desired percentage is reached.

$$
0 \%
$$
$100 \%$

How willing are you to take risks as a farmer?

\begin{tabular}{ccccc}
\hline $\begin{array}{l}\text { Not willing } \\
\text { to take risks }\end{array}$ & & & Very willing \\
to take risks
\end{tabular}


The following statements address your very personal attitude towards and use of modern technology. This is not about a single device, but about your attitude or your experience in the use of modern technologies/electronics in general.

Please mark with a cross.

\begin{tabular}{|c|c|c|c|c|c|}
\hline & $\begin{array}{l}\text { Totally } \\
\text { disagree }\end{array}$ & & & & $\begin{array}{c}\text { Totally } \\
\text { agree }\end{array}$ \\
\hline I am very curious about new technical innovations. & 1 & 2 & 3 & 4 & 5 \\
\hline Technical innovations mostly overwhelm me. & 1 & 2 & 3 & 4 & 5 \\
\hline $\begin{array}{l}\text { I find it difficult to deal with new technology - as } \\
\text { a rule, I simply do not know how to do that. }\end{array}$ & 1 & 2 & 3 & 4 & 5 \\
\hline $\begin{array}{l}\text { It is in my hands whether I succeed in using tech- } \\
\text { nical innovations (...). }\end{array}$ & 1 & 2 & 3 & 4 & 5 \\
\hline $\begin{array}{l}\text { I am always interested in using the latest technical } \\
\text { devices. }\end{array}$ & 1 & 2 & 3 & 4 & 5 \\
\hline $\begin{array}{l}\text { When dealing with modern technology, I am often } \\
\text { afraid of failing. }\end{array}$ & 1 & 2 & 3 & 4 & 5 \\
\hline $\begin{array}{l}\text { [I] would (...) use much more frequently technical } \\
\text { products than I currently do. }\end{array}$ & 1 & 2 & 3 & 4 & 5 \\
\hline $\begin{array}{l}\text { I'm afraid I'll be more likely to break down tech- } \\
\text { nological innovations (...). }\end{array}$ & 1 & 2 & 3 & 4 & 5 \\
\hline $\begin{array}{l}\text { What happens when I deal with [technology] is (...) } \\
\text { under my control. }\end{array}$ & 1 & 2 & 3 & 4 & 5 \\
\hline $\begin{array}{l}\text { I quickly find interest in new technical develop- } \\
\text { ments. }\end{array}$ & 1 & 2 & 3 & 4 & 5 \\
\hline
\end{tabular}

This question serves as a quality check.

Please tick "totally agree".

Totally disagree

1

$\square$
2

$\square$
3

$\square$
Totally agree

4

5

$\square$

\section{Evaluation autonomous field robots (AFR)}

How do you assess the use of AFR concepts on your farm?

Please tick only one box.

- I oppose the use of AFR (1)

- I fundamentally refuse the use of AFR (2)

- I am torn about the use of AFR (3)

- I am indifferent to the use of AFR (4)

- I would tolerate the use of AFR (5)

- I would accept the use of AFR (6) 
- I approve the use of AFR (7)

- I promote the use of AFR (8)

How would you rate the following statements about AFR if you imagined handling such machines?

Please mark with a cross.

\begin{tabular}{|c|c|c|c|c|c|}
\hline & $\begin{array}{l}\text { Totally } \\
\text { disagree }\end{array}$ & & & & $\begin{array}{c}\text { Totally } \\
\text { agree }\end{array}$ \\
\hline $\begin{array}{l}\text { AFR could lighten my workload } \\
\text { in certain operations. }\end{array}$ & 1 & 2 & 3 & 4 & 5 \\
\hline AFR enables me to save work force. & 1 & 2 & 3 & 4 & 5 \\
\hline I imagine the operation of AFR to be difficult. & 1 & 2 & 3 & 4 & 5 \\
\hline I find AFR intimidating. & 1 & 2 & 3 & 4 & 5 \\
\hline I would trust AFR to make the right decisions. & 1 & 2 & 3 & 4 & 5 \\
\hline Using AFR, I would be afraid to damage it. & 1 & 2 & 3 & 4 & 5 \\
\hline With AFR, I could operate more efficiently. & 1 & 2 & 3 & 4 & 5 \\
\hline A safe handling of AFR would be difficult for me. & 1 & 2 & 3 & 4 & 5 \\
\hline $\begin{array}{l}\text { If I were to use AFR, I would be afraid of misusing } \\
\text { it. }\end{array}$ & 1 & 2 & 3 & 4 & 5 \\
\hline $\begin{array}{l}\text { AFR would make farming more environmentally } \\
\text { friendly. }\end{array}$ & 1 & 2 & 3 & 4 & 5 \\
\hline $\begin{array}{l}\text { Learning how to handle AFR would be difficult for } \\
\text { me. }\end{array}$ & 1 & 2 & 3 & 4 & 5 \\
\hline $\begin{array}{l}\text { I think that other farmers would like to see AFR } \\
\text { used on my farm. }\end{array}$ & 1 & 2 & 3 & 4 & 5 \\
\hline $\begin{array}{l}\text { I would follow the instructions given to me by an } \\
\text { AFR. }\end{array}$ & 1 & 2 & 3 & 4 & 5 \\
\hline $\begin{array}{l}\text { The maintenance/repair of AFR would be a problem } \\
\text { on my farm. }\end{array}$ & 1 & 2 & 3 & 4 & 5 \\
\hline I find AFR frightening. & 1 & 2 & 3 & 4 & 5 \\
\hline $\begin{array}{l}\text { I think it would make a good impression on society } \\
\text { if I used AFR. }\end{array}$ & 1 & 2 & 3 & 4 & 5 \\
\hline
\end{tabular}


In summary, I consider the use of AFR to be...

Please mark with a cross.

a bad idea$$
1
$$

$\square$

an unwise move

$$
1
$$

$\square$

negative

1

$\square$
2

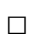

2

3

口

3

口 a good idea

4

5

口

a smart move

4

5

positive

4

5

$\square$ 


\section{I.4 German Farmers' Intention to Use Autonomous Field Robots: A PLS Analysis}

Friedrich Rübcke von Veltheim, Ludwig Theuvsen und Heinke Heise

Geleisteter Eigenanteil an dem Beitrag

\begin{tabular}{|c|c|c|c|c|c|}
\hline $\begin{array}{l}\text { Literatur- } \\
\text { recherche }\end{array}$ & $\begin{array}{l}\text { Entwicklung } \\
\text { des } \\
\text { Forschungs- } \\
\text { rahmens }\end{array}$ & $\begin{array}{l}\text { Konzept und } \\
\text { Design der Stu- } \\
\text { die }\end{array}$ & $\begin{array}{l}\text { Auswahl, Aus- } \\
\text { führung und } \\
\text { Entwicklung } \\
\text { von Methoden }\end{array}$ & $\begin{array}{l}\text { Interpretation } \\
\text { der } \\
\text { Ergebnisse }\end{array}$ & $\begin{array}{l}\text { Konzept und } \\
\text { Schreiben des } \\
\text { Artikels }\end{array}$ \\
\hline$>90 \%$ & $>75 \%$ & $>75 \%$ & $90 \%$ & $>75 \%$ & $>75 \%$ \\
\hline
\end{tabular}

Dieser Beitrag ist so oder in ähnlicher Fassung zur Veröffentlichung in der wissenschaftlichen Zeitschrift „Precision Agriculture“ angenommen. 


\section{Inhalt}

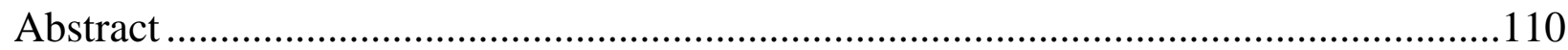

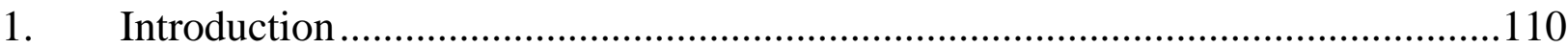

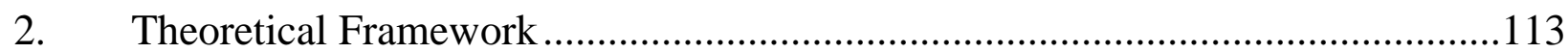

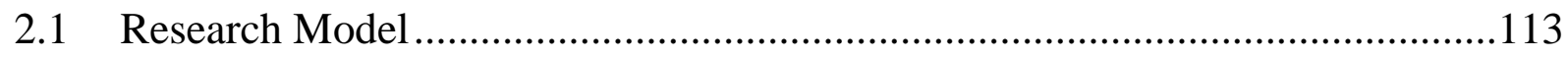

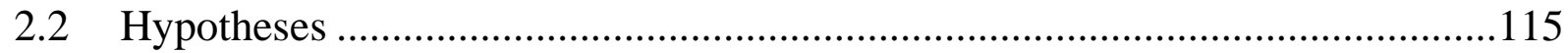

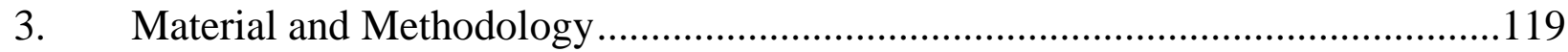

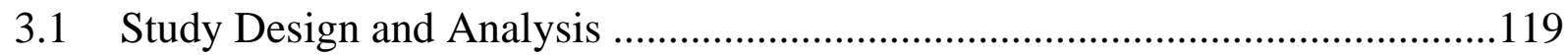

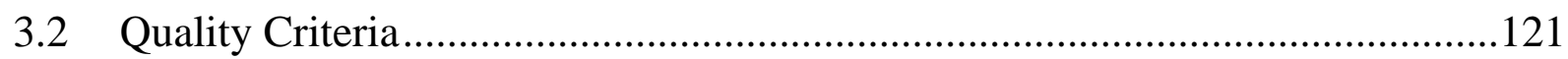

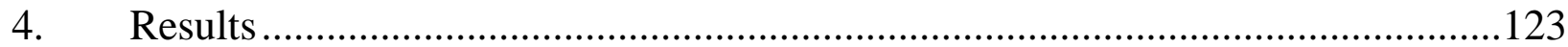

4.1 Sample Description and Descriptive Results ......................................................123

4.2 Evaluation of Quality Criteria of the Model ........................................................126

4.3 Hypothesis Verification.............................................................................127

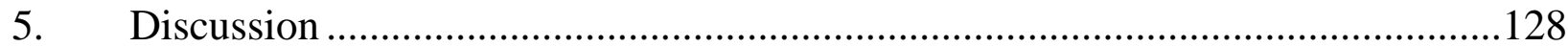

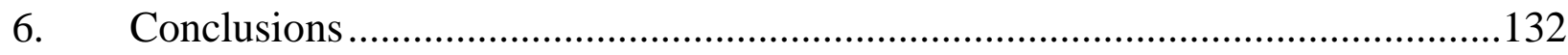

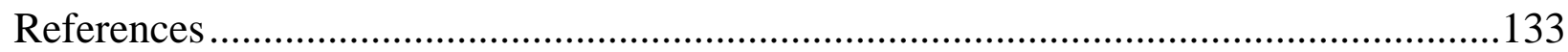

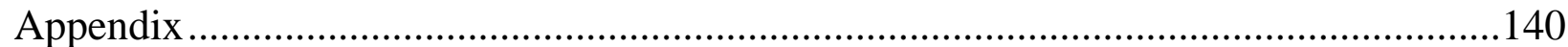




\begin{abstract}
AFR are a promising technology for solving several problems in agriculture, as they are electrical driven, can control weeds single plant based mechanically or with microdoses of pesticides and exert less ground pressure on the field. Whether such robots will be applied on a large scale in German agriculture depends on various parameters. Therefore, the factors influencing the behavioural intention of farmers with respect to their future adoption of AFR were investigated. The analysis applies a structural equation model (SEM) based on an extended version of the UTAUT. The dataset, collected in 2019, consists of 500 German farmers. The results reveal significantly positive effects of farmers' expected performance, social influence, and trust as well as significantly negative effects of farmers' effort expectancy and anxiety on the behavioural intention to use AFR. Additionally, moderating effects of age on the relationship of individual constructs to the behavioural intent to use robots could be confirmed. The results provide important information for various stakeholders. Robot suppliers should better inform farmers about the performance of their products, for instance by involving farmers in the development process of the robots. The ecological benefits attributed to field robots could meet public expectations and should be better communicated to address farmers' social influence on the behavioural intention to use the robots. Policymakers could try to create better framework conditions, for example by establishing a stable legal situation for autonomous systems or promote its use.
\end{abstract}

\title{
Keywords
}

Autonomous field robots, farmer, Germany, partial-least-squares method, Unified Theory of Acceptance and Use of Technology model

\section{Introduction}

In the course of digitalization, various SFT are currently established in developed countries. In addition to economic benefits such as increased production at lower input costs, they promise a more environmentally friendly farm management (LUTZ, 2017). One of the most recent developments in this area are AFR. AFR are defined in this paper as robots that act autonomously in a goal-directed manner in an automation-averse environment, i.e., an environment that is not controlled and not fully known, according to automatic interpretations of sensor data from that environment (HERTZBERG, 2014). In this context, the focus rests on small-machine AFR concepts. This technology could help to improve the 
efficiency and sustainability of current plant production by mechanically treating weeds without an operator or chemically treating plant diseases with pesticides in microdoses (KING, 2017). This is of particular interest to farmers in Germany because, on the one hand, the range of chemical crop protection agents is becoming ever smaller and, on the other hand, the legal framework for applying pesticides is becoming increasingly restrictive. The elimination of a driver would also increase work safety when handling pesticides by further reducing human contact with these substances. In addition, given the increasing shortage of skilled workers, driverless systems are an advantage for farmers, especially in laborintensive areas such as the cultivation of specialty crops. Due to the relatively low weight of AFR, soil compaction would substantially be reduced (MINßEN et al., 2015). At the same time, small AFR are more scalable than large farm equipment, which could make smallholder farms economically attractive again (KING, 2017). Since most AFR concepts are powered by electricity, less noise and pollutants would be emitted as well, which would lead to less annoyance among residents, especially when operating close to residential areas. KUCZERA (2006) has demonstrated that farmers are important members in rural communities, and they are influenced by their social environment in their actions. Therefore, they should also be interested in reducing social tensions regarding agriculture.

Nevertheless, various scientists have suggested possible cognitive barriers with the introduction of AFR which could lead to acceptance problems among farmers. The shift from conventional agricultural machinery to highly autonomous unmanned vehicles presents a new scenario of robots working out of the sight of farmers, which some farmers might be sceptical about (REDHEAD et al., 2015). REDHEAD et al. (2015) point out that Australian farmers fear the degree of complexity of AFR, the lack of staff qualified to operate this new technology and limited access to reliable commercial communication and data storage services. In addition, DEVITT (2018) revealed cognitive barriers to the acceptance of AFR by farmers. She fears that a lack of confidence in autonomous technology, concerns about losing agricultural skills or the loss of social recognition may have a negative impact on the behavioural intention to use AFR. This in turn is countered by falling labour costs and higher harvest expectations (RIAL-LOVERA, 2018). KESTER et al. (2013) were able to confirm for German farmers that although there is a basic willingness to adopt AFR, a certain scepticism about the safety and reliability of such systems exists.

However, since the step from prototype AFR concepts to market-ready products has recently been done, it has become clear that autonomous technology concepts will find their way into agriculture. Nevertheless, it is still unclear how and to what extent this will happen. While Australia was planning the commercial distribution of AFR in 2020, forecasts 
for Germany predict a broader commercialization of AFR towards 2030 (PICKEL, 2019). Despite such predictions and due to its disruptive potential, a successful introduction of AFR into agriculture requires its acceptance by society, but also by individual stakeholders, such as farmers or agricultural machinery manufacturers (BRAUER, 2017; REDHEAD et al., 2015; VENKATESH et al., 2003). Following WEIDNER et al. (2015), among various other parameters, such as the economic feasibility of AFR (i.e., LOWENBERG-DEBOER et al., 2020; SHOCKLEY et al., 2019), national policies and the legal situation (BASU et al., 2020) for autonomous systems resulting from these policies, an understanding of the various factors determining farmers' acceptance of AFR is needed to provide further information about a potential success of this new technology. This is all the more true as farmers tend to follow the principle of bounded rationality (SIMON, 1956) in their decision-making behaviour in contrast to the consistently rational "homo economicus" (MUßHOFF et al., 2009). Since AFR have not yet been commercialised on a large scale, there will hardly be any experience of using AFR among farmers (RÜBCKE VON VELTHEIM et al., 2019). According to VENKATESH et al. (2003) the use behaviour of a new technology is determined by the behavioural intention, which in turn is influenced by various factors (see "Theoretical framework" section). The link between behavioural intention and subsequent adoption has been partially supported by studies of technologies that are part way through the adoption process (e.g., AMBONG and PAUlino, 2020; AMIN and LI, 2014; FAR and RAZAEIMOGHADDAM, 2015; SCHUKAT et al., 2019). To this end, data were usually collected from different farmers (AMBONG and PAULINO, 2020; SCHUKAT et al., 2019) or agricultural experts (FAR and REZAEI-MOGHADDAM, 2014) via surveys or focus group discussions (AMIN and LI, 2013) and analysed using partial least squares (PLS) models. A PLS model combines path analysis, principal component analysis and regression. In detail, it investigates how far the latent variables can be described by indicators or manifest variables (HORNBURG and GIERING, 1996). In the absence of studies, yet there is no empirical evidence of the factors driving or prohibiting the intention to use AFR (REDHEAD et al., 2015; RIALLOVERA, 2018). Thus, the results of this study can contribute to the discussion on autonomous solutions in agriculture and serve as decision support for various stakeholder groups such as agricultural technology manufacturers, policymakers, technology acceptance researchers and farmers.

Therefore, the aim of this study is a first quantification of the determinants influencing the behavioural intention to use AFR. For this purpose, a research model is developed based on the UTAUT according to VENKATESH et al. (2003) and taking into account the findings of DEVITT (2018), which will be discussed in more detail in the following. After a 
description of the methodological approach, the model is applied to an online survey of German farmers $(n=500)$ and finally analysed using a PLS SEM. After the presentation of the results, the paper concludes with a discussion of the results and conclusions.

\section{Theoretical Framework}

\subsection{Research Model}

For the ex-ante assessment of user acceptance of AFR among farmers, the UTAUT model according to VENKATESH et al. (2003) was chosen from the many existing acceptance models. The UTAUT model is a combination of previous findings from well-documented acceptance research models and was specifically designed to predict technology acceptance (VENKATESH et al., 2003). It is thus more specific and clarifies more variance in technology acceptance than many of the initial models, such as the theory of planned behaviour (TPB) or the technology acceptance model (TAM) (AJZEN, 1991; AJZEN, 2005; DAVIS, 1989; VENKATESH et al., 2003). In addition, UTAUT is characterized by its generalizability to different forms of new technology and its transferability to different situations (BRAUER, 2017). Consequently, it has also been successfully applied in various studies addressing issues in the agricultural context (e.g., for SFT: CHIKOYE et al., 2018). The UTAUT also formed the basis for acceptance studies for autonomous robots in the nonagricultural sector, e.g., in the context of care robots (TURJA et al., 2020). Thus, a transfer to the analysis of the use of AFR in agriculture seems justified.

According to the UTAUT model, the use behaviour, which indicates the actual use of a new technology, in this case the use of AFR, is determined by the two variables behavioural intention and facilitating conditions. Both behavioural intention and use behaviour, which are described in more detail below, are dependent variables in this model. Since AFR have not widely been introduced into the market, it does not seem to be reasonable to investigate the actual use behaviour of farmers. Hence, this investigation is focused on behavioural intention, which is determined by the independent key constructs: performance expectancy, effort expectancy and social influence. In addition, the variables gender, age, experience, and voluntariness can have a moderating effect on the relationships between these constructs and the behavioural intention, whereby, according to VENKATESH et al. (2003), voluntariness is not seen as a requirement for acceptance research. Furthermore, age and experience can also have moderating effects on the relationship between the construct facilitating conditions and use behaviour (VENKATESH et al., 2003). 
In order to adapt the UTAUT model to the context of AFR in agriculture and due to the results of previous studies, the independent variables trust, anxiety and technology commitment (subdivided into the three facets technological interest, technological competence beliefs and technological control beliefs) were added to the key constructs (D'ANTONI et al., 2012; DEVITT, 2018; HEERINK et al., 2010; KUlviwAt et al., 2007; KUTTER et al., 2011; MCALLISTER, 1995; NEYER et al., 2012; VENKATESH et al., 2011) (see Table A1 in the appendix). The added construct technology commitment complements the UTAUT model with a satisfactorily validated psychometric construct that takes up the use of new technology in general (NEYER et al., 2012). Technology commitment is composed of the three facets, i.e., technological interest, technological competence beliefs and technological control beliefs. The variables trust and anxiety were included based on their empirically proven influence on the intention to use a new technology (HEERINK et al., 2010; KULVIWAT et al., 2007; VENKATESH et al., 2011). Trust is a major condition for the success of products (MACMILLAN et al., 2005). It influences the way people interact with other people and whether they are willing to delegate tasks to another agent - no matter if the agent is human or artificial. In this study, trust describes the belief in AFR's performance with personal integrity and reliability (HEERINK et al., 2010), because it leads to appropriate reliance on the technology enhancing safety and performance issues, while a lack of trust in new technologies can pose a cognitive acceptance barrier (DEVITT, 2018). According to BREHM (1966), anxiety can be seen as the counterpart to trust and, in comparison, has a negative connotation. HEERINK et al. (2010) defines anxiety as: "evoking anxious or emotional reactions when it comes to using the system" and assumes an influence in the UTAUT model based on NOMURA et al. (2006). Since farmers are free to decide on the use of AFR and are not subject to any constraints and according to VENKATESH et al. (2012), who did the same in an extension (UTAUT 2) of the UTAUT model, the moderator voluntariness was not included in the research model of this paper. Finally, since the commercialization of AFR on large scale has not yet started (RÜBCKE VON VELTHEIM et al., 2019), the original construct use behaviour, which describes the actual usage of AFR, as well as its corresponding construct facilitating conditions were excluded.

The following Figure 1 summarizes all hypotheses of this study. The three constructs, which were added in order to adapt the UTAUT model to this study, are highlighted in bold letters. 


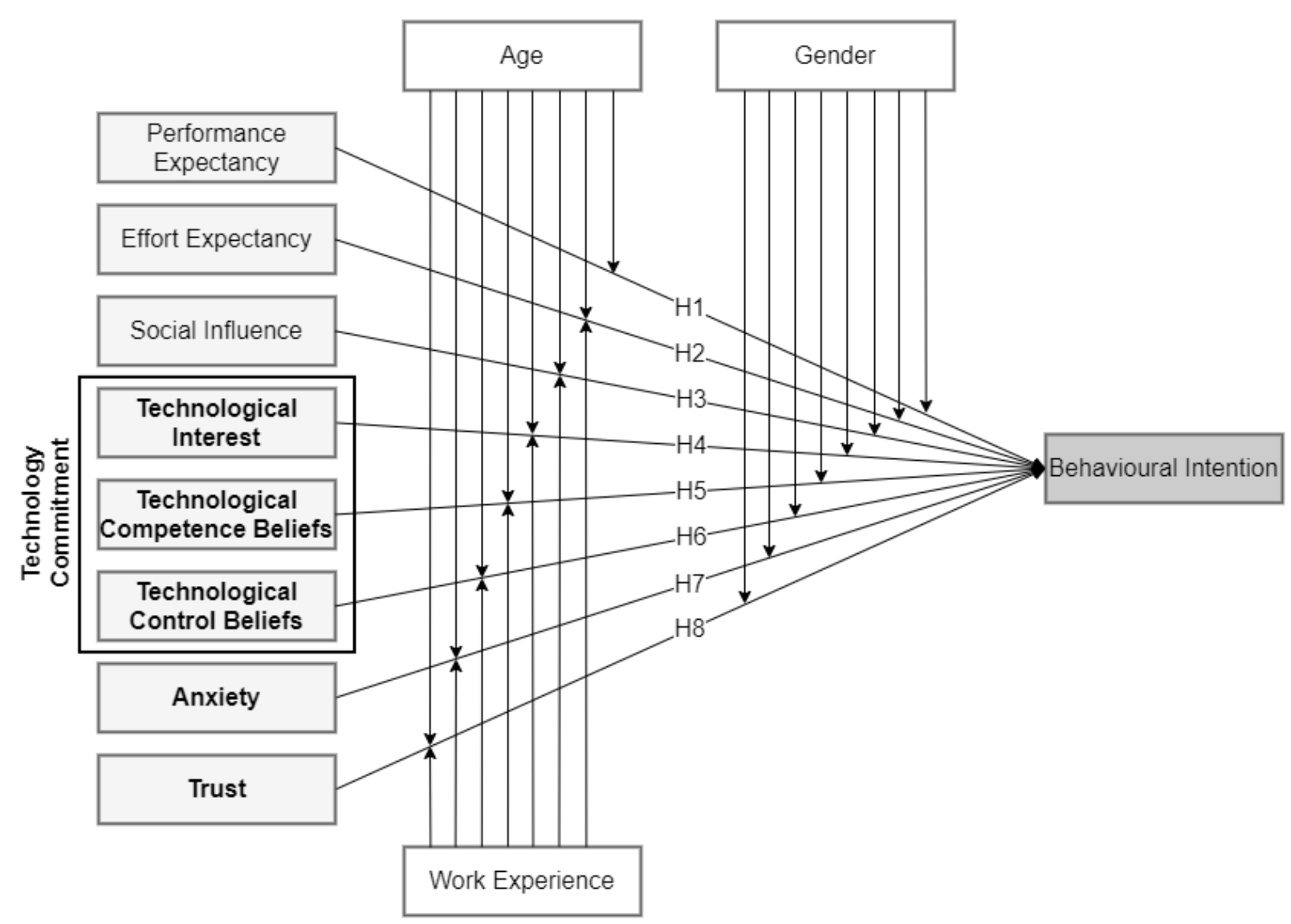

Figure 1: Research model, adapted from VENKATESH et al. (2003); bold letters = added variable

\section{Source: Authors' illustration}

\subsection{Hypotheses}

The behavioural intention indicates the requests and efforts of a person to perform behaviour to use a new technology. In this study, behavioural intention measures the attitude of potential users towards AFR usage, whereby it was assumed that the relation between the independent variables and the behavioural intention is moderated by gender, age, and work experience. The determinants are explained below, and hypotheses formulated.

Performance expectancy refers to the assessment by a potential user of the extent to which the use of a new technology improves the performance of the work within a certain context, in this case arable farming. In many technology acceptance studies, performance expectancy has the strongest influence on the behavioural intention and its relation is often moderated by gender and age (MORRIS and VENKATESH, 2000; VENKATESH et al., 2003; VENKATESH and MORRIS, 2000). Economic performance plays an important role in investment decisions, e.g., adopting AFR (SHOCKLEY et al., 2019). VENKATESH et al. (2003) assume that given the fact with men being highly task-oriented (MINTON and SCHNEIDER, 1980), performance expectations that focus on the fulfilment of tasks are also likely to be 
particularly evident for men. With regard to man-dominated agriculture (EUROSTAT, 2019), an influence of the gender might be demonstrated. Therefore, it was assumed that gender has a moderating effect on the relationships between the key constructs (performance expectancy, effort expectancy, social influence, technology commitment, anxiety, and trust) and the behavioural intention. TAMIRAT et al. (2017) showed for agriculture that younger farmers are more willing to use PAT, such as AFR, based on increased interest in new technologies. In addition, work experience could also have a moderating effect on the relationship between the key constructs and behavioural intention, as experienced farmers may have different views than inexperienced potential users (LEVY, 1988). For this reason, the following hypotheses were derived:

H1: Performance expectancy has an influence on the behavioural intention to use AFR.

H1a: The effect of performance expectancy on behavioural intention is moderated by age and gender.

Effort expectancy is the degree to which a user believes that a system will be free of effort or difficult to operate. It covers not only financial but also time aspects and is often perceived as higher in a new system at an early stage of implementation. It is predicted to be more challenging for older women with less work experience than for men (BEM and ALLEN, 1974; Bozionelos, 1996; VenKATESH and MorRis, 2000). Plude and Hoyer (1985) demonstrated that with increasing age the processing of complex information that may be necessary for the operation of AFR becomes more difficult. The more experienced a user is in handling technology the less effort is expected from a new technology (LEVY, 1988). For these reasons, gender, age, and work experience were expected to have a moderating effect on the relationship between the perceived ease of use and the behavioural intention regarding AFR (GLOY and AKRIDGE, 2000; HOAG et al., 1999; VENKATESH et al., 2003). The following hypotheses were derived:

H2: Effort expectancy has an influence on the behavioural intention to use AFR.

H2a: The effect of effort expectancy on behavioural intention is moderated by age, gender, and work experience.

The social influence refers to the perception of whether people relevant to the potential user believe that the potential user should use the new technology, in this case AFR. This effect also decreases with greater experience (VENKATESH and DAVIS, 2000). Regarding agriculture, KUCZERA (2006) identified that colleagues, friends, and family members have a social influence on strategic farm decisions. Furthermore, it was observed that farmers' 
neighbours' experience with new technologies has a significant effect on future use (FoSTER and ROSENZWEIG, 1995). Gender, age, and experience were identified as moderating variables. On the one hand, the literature points out that women react more sensitively to the opinions of others and thus their intention to use AFR is easier to influence (VENKATESH et al., 2003). On the other hand, RHODES (1983) described an increased need for belonging among older people, so that increased social influence was assumed. Both described influences decrease with increasing experience with technology (VENKATESH and MORRIS, 2000).

H3: Social influence affects the behavioural intention to use AFR.

H3a: The effect of social influence on behavioural intention is moderated by age, gender, and work experience.

Technology commitment complements the UTAUT model with a psychometric construct that reflects the use of new technology in general (NEYER et al., 2012). Various studies have shown that younger farmers in particular are more committed to PAT than older ones (D’ANTONI et al., 2012; KUTTER et al., 2011). In addition, studies on gender differences revealed that young men are more interested in and ready for new technology than women (VENKATESH et al., 2003). It was assumed that this applies also to the relation between technology commitment and the behavioural intention to use AFR. The technology commitment is composed of the three facets technological interest, technological competence beliefs and technological control beliefs.

Following DAVIS (1989), NEYER et al. (2012) define technological interest as a representative attribute that reflects the subjective evaluation of technological progress. It is well documented that farmers use new production methods when they have a high technological interest (AUSTIN et al., 1998). This is all the more relevant when they are going to pioneer a new technology (Voss et al., 2008). It was therefore be assumed that a potential user with a high technological interest is much more willing to use AFR than a potential user with a more technological aversion.

H4: Technological interest has an influence on the behavioural intention to use AFR.

H4a: The effect of technological interest on behavioural intention is moderated by age, gender, and work experience.

Technological competence beliefs are defined by NEYER et al. (2012) based on the concept of competence beliefs by KRAMPEN (1991) as the subjective expectation of opportunities in technology relevant situations. It also reflects the expected adaptability to still unknown technological innovations. Since AFR has not yet been widely commercialized, most 
potential users may not have gained experience with this new technology. Therefore, it was assumed that technological competence beliefs have an influence on the behavioural intention.

H5: Technological competence beliefs have an influence on the behavioural intention to use AFR.

H5a: The effect of technological competence beliefs on behavioural intention is moderated by age, gender, and work experience.

Technological control beliefs, as the final of the three facets of technology commitment, were also defined in reference to KRAMPEN (1991) as individual contingency expectations, which represent the subjective expectation of the results of actions relevant to technology (BEIER, 1999). They reflect the level of perceived controllability of technology. Following DEVITT (2018), potential AFR users in the initial stage of adoption are often willing to put in a lot of energy to try and make the new technology work. At this stage, a poor user interface design that makes set up laborious, complex, or confusing is one of the greatest barriers to the adoption of AFR. In addition, SFT, such as AFR, are characterized by a large amount of collected data that is often beyond the control of potential users, which could have an influence on the behavioural intention.

H6: Technological control beliefs have an influence on the behavioural intention to use AFR.

H6a: The effect of technological control beliefs on behavioural intention is moderated by age, gender, and work experience.

Where new technologies such as AFR are introduced, trust leads to a certain degree of dependency on the technology, which causes safety and performance problems, while a lack of trust in new technologies may act as a cognitive barrier to acceptance (DEVITT, 2018). KRAMPEN et al. (1982) stated that women often have more trust than men and that trust decreases with age. In addition, GALLIMORE et al. (2019) found that women report greater trust and perceived trustworthiness of autonomous robots relative to men. Taken the above together, the following was hypothesized:

H7: Trust has an influence on the behavioural intention to use AFR.

H7a: The effect of trust on behavioural intention is moderated by age, gender, and work experience.

In the light of the aforementioned studies by GALLIMORE et al. (2019) and KRAMPEN et al. (1982) and in addition to the findings of GHAZALI et al. (2018), who reported that women 
evidenced lower anxiety to a male robot relative to a female robot, it was assumed that the construct anxiety also differs in terms of gender and age. Hence, it was hypothesized that it also has an influence on the behavioural intention to use AFR.

H8: Anxiety has an influence on the behavioural intention to use AFR.

H8a: The effect of anxiety on behavioural intention is moderated by age, gender, and work experience.

\section{Material and Methodology}

\subsection{Study Design and Analysis}

In July and August 2019, a standardized online survey of farmers $(n=500)$ was performed in Germany in order to assess farmers' behavioural intention to use AFR as well as its determinants. Personalised links for the online survey were used to ensure that each participant could only answer the questionnaire once. The survey was pre-tested for two weeks and was finally implemented with the help of the Unipark software (Globalpark AG). The attributes of the individual questions and statements were measured using five-point Likert scales (from 1 "fully disagree" to 5 "fully agree"). In order to assure a consistent knowledge base about AFR among the participating farmers, a three-minute, informative video on how AFR work and the possible areas of application was presented in the beginning of the questionnaire. Additionally, control questions were established for quality assurance. Furthermore socio-demographic and farm characteristics were inquired. For the recruitment of survey participants, various channels were used, such as the mailing lists of the German Farmers' Association, the publication of the survey link on social media pages of various agricultural journals, an interview with $\mathrm{f} 3$ magazine, and the use of the published mailing lists of farms, certified to train farmers, as well as private contacts. After eliminating incomplete datasets, 533 complete questionnaire replies could be compiled. However, further observations had to be excluded from analysis. The exclusion criteria were unsystematic response behaviour, processing time that deviated significantly from the average (18 minutes) and an incorrect answer to the quality control question. After the dataset had been cleared of incorrectly completed forms, 500 observations remained for further investigation.

The data analysis was done based on the extended UTAUT model (see "Theoretical framework" section). All constructs described above, which will be investigated in relation to the behavioural intention to use AFR, are latent variables. Latent variables cannot be observed directly and must therefore be described with the help of empirical indicators 
(HORNBURG and GIERING, 1996). All empirical indicators which were used to describe the latent variables are illustrated in Table A1 in the appendix. To further analyse the relationships of the latent variables, the component based PLS SEM was used. A PLS SEM consists of two elements: a structural model, which displays the relationships between the constructs, and a measurement model, which displays the relationships between constructs and indicators (HAIR et al., 2016). The measurement model can either be formative or reflective (DiAMANTOPOULOS, 2011). In this paper a reflective measurement model is used. This was done because the individual indicators of the respective constructs are highly significantly correlated with each other and the exclusion of one indicator does not trigger a sizeable effect on the associated construct (e.g., HAIR et al., 2016). The model differentiates between dependent (endogenous) and independent (exogenous) latent variables (HENSELER, 2005). PLS is characterized by its suitability for complex models and explorative studies and was therefore chosen for the present analysis.

To test the moderating effects of gender, age, and work experience (H1a - H8a), multigroup analysis (MGA) were performed because they allow testing a moderator continuously throughout the research model (HENSELER and CHIN, 2010). Further, MGA is very well suited to calculate the strength of the moderating effects (BARON and KENNY, 1986; SARSTEDT et al., 2011). It should be noted that a moderator can change not only the strength but also the direction of a relationship in the research model. Two separate bootstraps are calculated and compared for the MGA (HENSELER and CHIN, 2010). The estimated parameters from the bootstrap calculation help to check the probability of differences between groups. If there is a group difference that is significant, a moderating effect can be assumed (HENSELER et al., 2009). For age and work experience a median split was performed to divide the moderators into two groups. The younger group included everyone born after 1978; the older group included everyone born before 1979. The less experienced group included those with less than 22 years of work experience; the experienced group included those with more than 21 years of work experience. Gender was divided in male and female. Evaluations were performed with the program SmartPLS v. 3.2.8. 


\subsection{Quality Criteria}

The analysis of the PLS model is based on a two-stage approach: First, the quality of the structural model is evaluated with regards to its reliability and validity; second, the measurement model is checked. Since the objective of PLS SEM is the maximization of the explained variance for the endogenous latent variables, in this study the behavioural intention to use AFR, the quality evaluation of the measurement model as well as of the structural model concentrate on key figures for the PLS path model's predictive power (HAIR et al., 2016). For the reflectively specified measurement model, this includes internal consistency reliability, convergent and discriminant validity. The internal consistency reliability indicates how well the individual indicators explain the underlying construct. It is evaluated using the two criteria composite reliability and Cronbach's alpha. The composite reliability should be higher than 0.7 , whereby values above 0.6 are also considered to be acceptable in explorative studies (HAIR et al., 2016). The same specifications apply to Cronbach's Alpha. Composite reliability tends to overestimate the true value, whereas Cronbach's Alpha tends to underestimate it, so the true value may lie between these two criteria (HAIR et al., 2016).

To evaluate the convergent validity, which describes the extent to which a measurement is positively correlated with an alternative measurement of the same construct (HAIR et al., 2016), the two criteria: indicator reliability and average variance extracted (AVE) were used. Indicator reliability of a construct reveals how much the associated indicators have in common, whereas AVE describes how much of the variance in an indicator is explained by the construct (HAIR et al., 2016). For indicator reliability, the loading of an indicator should be above 0.7, whereas the AVE should be above 0.5 , so that the construct explains at least $50 \%$ of each indicator's variance (HULLAND, 1999). The discriminant validity is the extent to which one construct differs from another construct (HAIR et al., 2016). Its measurement is thus concerned with the empirical validity of a construct. To determine the discriminant validity, the Fornell-Larcker criterion, cross loadings and the relatively new heterotrait-monotrait ratio (HTMT) were used. The Fornell-Larcker criterion compares the square root of the AVE values with the latent variable correlations and tests whether a construct shares more variance with its associated indicators than with any other construct (FORNELL and LARCKER, 1981). To fulfil the criteria, the Fornell-Larcker criterion should be larger than the AVE. To further test if the discriminant validity has been established, an indicator's reliability on the associated construct should be greater than any other of its cross-loadings (CHIN, 1998). Otherwise, an indicator should be removed (HAIR et al., 2016). Following Henseler et al. (2015), the Fornell-Larcker criterion and cross-loadings 
alone are not sufficient to fulfil the discriminant validity criteria. Therefore, the HTMT is needed, which describes the relationship between indicators that measure different constructs, and between indicators that each measures its own construct (HAIR et al., 2016). To have no lack of discriminant validity the HTMT should not exceed 0.85 , nor should the confidence interval contain the value 1 (HENSELER et al., 2015). The quality criteria described for the reflective measurement model are summarized in Table 1.

Table 1: Quality criteria of reflective measurement models

\begin{tabular}{ll}
\hline Quality criteria & Requirement \\
\hline Internal consistency reliability & $\begin{array}{l}\text { Composite reliability }>0.6 \text { and }<0.9 \\
\text { Cronbach's alpha }>0.6 \text { and }<0.9\end{array}$ \\
\hline Convergent validity & $\begin{array}{l}\text { Indicator reliability }>0.7 \\
\text { AVE }>0.5\end{array}$ \\
\hline Discriminant validity & $\begin{array}{l}\text { Fornell-Larcker criterion }>\text { AVE } \\
\text { Cross loadings }<\text { loadings on the associated constructs } \\
\end{array}$ \\
& HTMT of the correlations $<0.85 ;$ confidence interval $\neq 1$ \\
\hline
\end{tabular}

Source: Authors' illustration according to HAIR et al. (2016)

After the measurement model was tested, the results of the structural model were evaluated. This was done in six steps: 1) assess structural model for collinearity, 2) assess the significance and relevance of the structural model relationships, 3) assess the level of $\mathrm{R}^{2}, 4$ ) assess the $\mathrm{f}^{2}$ effect size, 5) assess the predictive relevance $\mathrm{Q}^{2}$, and 6) assess the $\mathrm{q}^{2}$ effect size (HAIR et al., 2016). To test collinearity in the structural model, the inner variance inflation factor (VIF) was used, which describes the degree to which the standard error has been increased due to the presence of collinearity (HAIR et al., 2016). If the VIF is above 5 ( $80 \%$ of the indicators' variance has been verified by the remaining indicators related with the same construct), a critical level of collinearity is indicated and an elimination of constructs, a predictor merge into a single construct or the creation of higher-order constructs should be considered in order to treat collinearity problems (HAIR et al., 2011). The significance and relevance of the structural model relationships was assessed through non-parametric bootstrapping. The bootstrapping method generates subsamples that are randomly drawn from the data set and after that are used to estimate the PLS path model (DAVISON and HINKLEY, 1997; EFRON and TIBSHIRANI, 1986). The p-values should be lower than 0.05 (significant), 0.01 (very significant) or 0.001 (highly significant). When determining the $\mathrm{R}^{2}$ value, it applies that the greater the $\mathrm{R}^{2}$, the higher the proportion of the declared variance (HAIR et al., 2016). The level of an acceptable $\mathrm{R}^{2}$ value depends on the research discipline. Thus, for some social scientists, a value of $\mathrm{R}^{2}$ greater than 0.25 is acceptable, which ensures that at least $25 \%$ of the variance of the endogenous variable is explained by the influencing factors (HAIR et al., 2011; HENSELER et al., 2009). The $\mathrm{f}^{2}$ value explains to 
which extent an exogenous latent variable contributes to the $\mathrm{R} 2$ value of an endogenous latent variable, where $\mathrm{f}^{2}$ values of 0.02 point to a small, 0.15 to a medium, and 0.35 to a large effect of an exogenous construct on an endogenous construct (COHEN, 1988). $\mathrm{Q}^{2}$ uses blindfolding procedure to assess the predictive relevance of a model (GöTZ et al., 2010). The division of the sample size by the omission distance (D) should not give an integer, where D should be selected between 5 and $10 . \mathrm{Q}^{2}$ values above 0 indicate a predictive relevance of the model to the endogenous construct (CHIN, 1998). In the last step the $\mathrm{q}^{2}$ effect size was assessed. It is a criterion for evaluating the relative predictive relevance of an exogenous construct to an endogenous construct and indicates a small (0.02), medium $(0.15)$ or large $(0.35)$ predictive relevance of an exogenous construct for an endogenous construct (HAIR et al., 2016).

\section{Results}

\subsection{Sample Description and Descriptive Results}

The descriptive statistics are shown in Table 2. The sample consists of $91.6 \%$ male and $8.4 \%$ female respondents. This is nearly representative for the gender ratio of farm managers in Germany ( $90.4 \%$ male; $9.6 \%$ female), as recorded in the last agricultural census in 2016 (EUROSTAT, 2019). The average age of the respondents is 41.5 years. In terms of age distribution, the sample is not representative for the population of those employed in agriculture in Germany in 2016. For example, farmers under the age of 35 are clearly overrepresented and farmers over the age of 44 are clearly under-represented in the sample (GERMAN FARMERS' ASSOCIATION, 2019). Work experience is relatively high, since about $67 \%$ of the respondents have worked in agriculture for more than 10 years. The majority of the farmers surveyed work in the regions North (Bremen, Hamburg, Lower Saxony, and Schleswig-Holstein: about 36 \%), East (Berlin, Brandenburg, Mecklenburg Western Pomerania, Saxony, Saxony-Anhalt, and Thuringia: about $20 \%$ ) and South-West (BadenWürttemberg, Rhineland-Palatinate, and Saarland: about $18 \%$ ). Except for the South-West region (around $22 \%$ national average) this does not match the national distribution of the farms in 2019. On a national average most farms are located in the South-East region (Bavaria: around $32 \%$ ) (DESTATIS, 2019). With respect to farm management practices, the sample represents approximately the national average for 2018, as $14.2 \%$ (national average: $12 \%$ ) of the respondents farm organically and $85.8 \%$ (national average: $88 \%$ ) conventionally (GERMAN FARMERS' ASSOCIATION, 2019). 
Table 2: Descriptive statistics $(n=500)$

\begin{tabular}{|c|c|c|c|c|}
\hline Variable & Description & Frequency & Percentage (\%) & German average $(\%)^{b}$ \\
\hline \multirow{2}{*}{ Gender $^{\mathrm{a}}$} & Male & 458 & 91.6 & 90.4 \\
\hline & Female & 42 & 8.4 & 9.6 \\
\hline \multirow{5}{*}{$\operatorname{Age}^{\mathrm{a}}$} & Under 25 & 52 & 10.4 & 7.7 \\
\hline & $25-34$ & 144 & 28.8 & 14 \\
\hline & $35-44$ & 89 & 17.8 & 16.4 \\
\hline & $45-54$ & 108 & 21.6 & 28.3 \\
\hline & Older than 55 & 107 & 21.7 & 33.6 \\
\hline \multirow{4}{*}{$\begin{array}{l}\text { Work experi- } \\
\text { ence }^{\mathrm{a}}\end{array}$} & Less than 10 years & 165 & 33.0 & n.a. \\
\hline & 10 to 20 years & 105 & 21.0 & n.a. \\
\hline & 21 to 30 years & 130 & 26.0 & n.a. \\
\hline & More than 30 years & 100 & 20.0 & n.a. \\
\hline North & $\begin{array}{l}\text { Bremen, Hamburg, } \\
\text { Lower Saxony, Schles- } \\
\text { wig-Holstein }\end{array}$ & 181 & 36.2 & 18.4 \\
\hline East & $\begin{array}{l}\text { Berlin, Brandenburg, } \\
\text { Mecklenburg Western } \\
\text { Pomerania, Saxony, } \\
\text { Saxony-Anhalt, Thurin- } \\
\text { gia }\end{array}$ & 101 & 20.2 & 9.6 \\
\hline West & $\begin{array}{l}\text { North Rhine-Westpha- } \\
\text { lia, Hesse }\end{array}$ & 80 & 16.0 & 18.0 \\
\hline South-West & $\begin{array}{l}\text { Baden-Württemberg, } \\
\text { Rhineland-Palatinate, } \\
\text { Saarland }\end{array}$ & 89 & 17.8 & 21.6 \\
\hline South-East & Bavaria & 49 & 9.8 & 32.5 \\
\hline \multirow{2}{*}{$\begin{array}{l}\text { Farm manage- } \\
\text { ment }\end{array}$} & Conventional & 429 & 85.8 & 88.0 \\
\hline & Organic & 71 & 14.2 & 12.0 \\
\hline \multirow[t]{9}{*}{ Farm size } & Less than 5 hectares & 4 & 0.8 & 8.1 \\
\hline & 5 to 9 hectares & 7 & 1.4 & 16.4 \\
\hline & 10 to 19 hectares & 18 & 3.6 & 20.2 \\
\hline & 20 to 49 hectares & 52 & 10.4 & 23.7 \\
\hline & 50 to 99 hectares & 99 & 19.8 & 17.5 \\
\hline & 100 to 199 hectares & 110 & 22.0 & 9.3 \\
\hline & 200 to 499 hectares & 102 & 20.4 & 3.3 \\
\hline & 500 to 1000 hectares & 51 & 10.2 & 0.9 \\
\hline & More than 1000 hectares & 57 & 11.4 & 0.6 \\
\hline
\end{tabular}

${ }^{\mathrm{a}}$ Gender, age and work experience classes were used as moderators in the UTAUT model

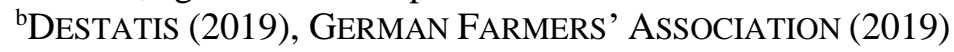

Source: Authors' calculations and illustration

In 2019, there were about 266,600 farms in Germany cultivating 16.7 million hectares of agricultural land, including 11.7 million hectares of arable land (DESTATIS, 2019). The farm size structure of the sample is shifted towards larger farms in comparison to the 
national average. Although most respondents farm 100 to 199 hectares in line with the national average, the class 200 to 499 hectares is overrepresented, and the classes 5 to 19 hectares is underrepresented (DESTATIS, 2019).

In general, the respondents seem to be positive about the use of AFR in agriculture (see Table A1 in the appendix). The performance expectancy scored the highest mean agreement $(\mu=4.07)$, followed by behavioural intention $(\mu=3.80)$, social influence $(\mu=3.32)$, trust $(\mu=3.27)$ and technology commitment $(\mu=3.20)$. Effort expectancy $(\mu=2.43)$ and anxiety $(\mu=1.79)$ received the lowest agreement. Within the construct perceived usefulness, the greatest benefit $(\mu=4.49 ; \sigma=0.729)$ was seen in the expected reduction of workload and the least benefit $(\mu=3.87 ; \sigma=0.945)$ in the more environmentally friendly management through the use of AFR. In terms of expected effort, the least difficulties $(\mu=2.87 ; \sigma=0.962)$ are associated with the safe handling of AFR, whereas slightly more problems are seen in the operation $(\mu=2.14 ; \sigma=0.850)$. Overall, on average more benefits $(\mu=4.07 ; \sigma=0.690)$ than efforts $(\mu=2.43 ; \sigma=0.710)$ are expected. With regard to the social influence, marginally more weight is given to the good impression on society through the use of AFR $(\mu=3.47 ; \sigma=0.938)$ than to the impression on other farmers $(\mu=3.25 ; \sigma=0.938$ ). The judgement of the farmers surveyed turned out to be clearer in the case of technology commitment. Here, the highest agreement is with the statement of being curious about new technologies $(\mu=4.42 ; \sigma=0.732)$, while farmers are least afraid of damaging these new technologies when using them $(\mu=1.58 ; \sigma=0.735)$. Trust in AFR is slightly higher in terms of them making the right decisions $(\mu=3.40 ; \sigma=0.910)$ compared to following up on these decisions $(\mu=3.16 ; \sigma=0.891)$. In contrast, there is slightly more concern about using AFR incorrectly $(\mu=2.24 ; \sigma=0.971)$, while almost none of the farmers surveyed find AFR intimidating $(\mu=1.43 ; \sigma=0.708)$.

The positive general orientation is also reflected in the dependent variable (behavioural intention) of the research model $(\mu=3.80)$. A more detailed understanding of the distribution across the individual indicators of behavioural intention is provided in Figure 2. Most of the farmers interviewed were interested in becoming familiar with the new technology during the first days of AFR use on their farm $(\mu=4.53)$ and learning how to use it properly $(\mu=4.60)$. However, farmers were rather indifferent to the implementation of AFR on their farms $(\mu=3.44)$, and there were no plans yet to use AFR in the nearest future $(\mu=2.70)$. The standard deviations range from $\sigma=0.722$ to $\sigma=1.163$. 


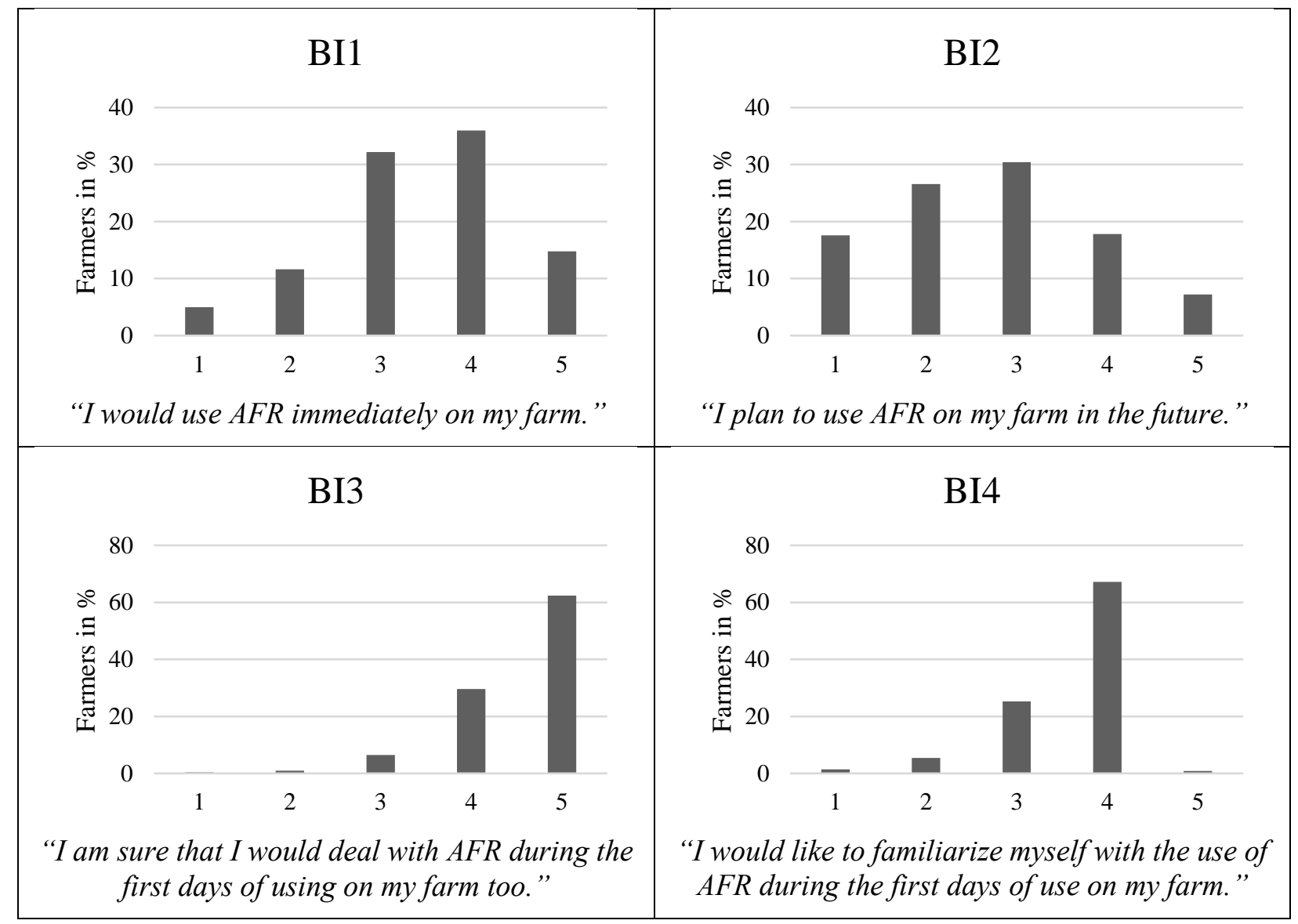

Figure 2: Response distribution in the construct behavioural intention. Scale form $1=$ totally disagree to $5=$ totally agree; $(n=500)$

\section{Source: Authors' illustration}

\subsection{Evaluation of Quality Criteria of the Model}

A PLS was used to analyse the extended UTAUT model and the derived hypotheses. First, the measurement model was evaluated by the quality criteria regarding reliability and validity as described in the "Material and methodology" section. The internal consistency reliability of each construct was assessed by Cronbach's alpha (CRA) and the composite reliability (CR). Table A1 (see appendix) shows that all CRA (ranging from 0.653 to 0.787 ) and CR (ranging from 0.783 to 0.858 ) values are above 0.6 and can therefore be considered acceptable for this explorative study. The convergent validity was computed using the indicator loadings and the AVE. To test the significance of the indicator loadings the bootstrap procedure was used with 5,000 subsamples. Most of the indicator loadings are above 0.7 (ranging from 0.657 to 0.910 ). Those indicators with a loading between 0.657 and 0.708 remained in the model, since its removal did not increase the composite reliability of the corresponding construct. All AVE values exceeded the threshold of 0.5 (ranging from 0.533 to 0.749 ). To determine the discriminant validity, the Fornell-Larcker criterion, cross loadings and the HTMT were checked. None of the observed AVE was higher than the 
squared correlations, so the Fornell-Larcker criterion was fulfilled (see A1 and A2 in the appendix). All indicators' cross loadings had a significantly higher loading on its own construct than on others, therefore no indicators had to be removed. The HTMT did not exceed 0.85 , nor did the confidence interval contain the value 1 , so that the discriminant validity can be assumed to be acceptable.

\subsection{Hypothesis Verification}

The results of the PLS analysis with standardized path coefficients are shown in Figure 3. Six out of nine hypotheses derived from the extended UTAUT model could be supported (H1, H2, H3, H4, H7, and H8). Performance expectancy $(0.314, \mathrm{p} \leq 0.001)$, social influence $(0.214, \mathrm{p} \leq 0.001)$, technological interest $(0.124, \mathrm{p} \leq 0.01)$ and trust $(0.132, \mathrm{p} \leq 0.001)$ had significantly positive effects on the behavioural intention to use AFR. A significantly negative influence on the behavioural intention to use AFR for effort expectancy $(-0.135$, $\mathrm{p} \leq 0.001)$ and anxiety $(-0.124, \mathrm{p} \leq 0.01)$ was observed. The effects of performance expectancy and social influence were the strongest. The research model accounted for $51.6 \%$ $\left(\mathrm{R}^{2}\right)$ of the variance in behavioural intention. For the gender and work experience, no moderating effect could be identified, whereas age was confirmed as a moderator. Regarding age, the moderating effect was confirmed for the two facets technological interest and technological competence beliefs of the technology commitment construct (see Table A4 in the appendix). In detail, there was almost no influence (0.016) of technological interest on behavioural intention for the younger group, whereas the older group showed a higher path coefficient (0.220). The opposite was observed for the technological competence beliefs. In this case the influence was negative and significantly higher in the younger group (0.178) than in the older group (0.013). According to CHIN (1998), path coefficients below 0.1 are not interesting to interpret although it is significant. As a result, the moderator hypotheses 1a, 2a, 3a, 6a, 7a, and 8a were not supported, whereas hypotheses $4 \mathrm{a}$ and $5 \mathrm{a}$ were partly supported. 


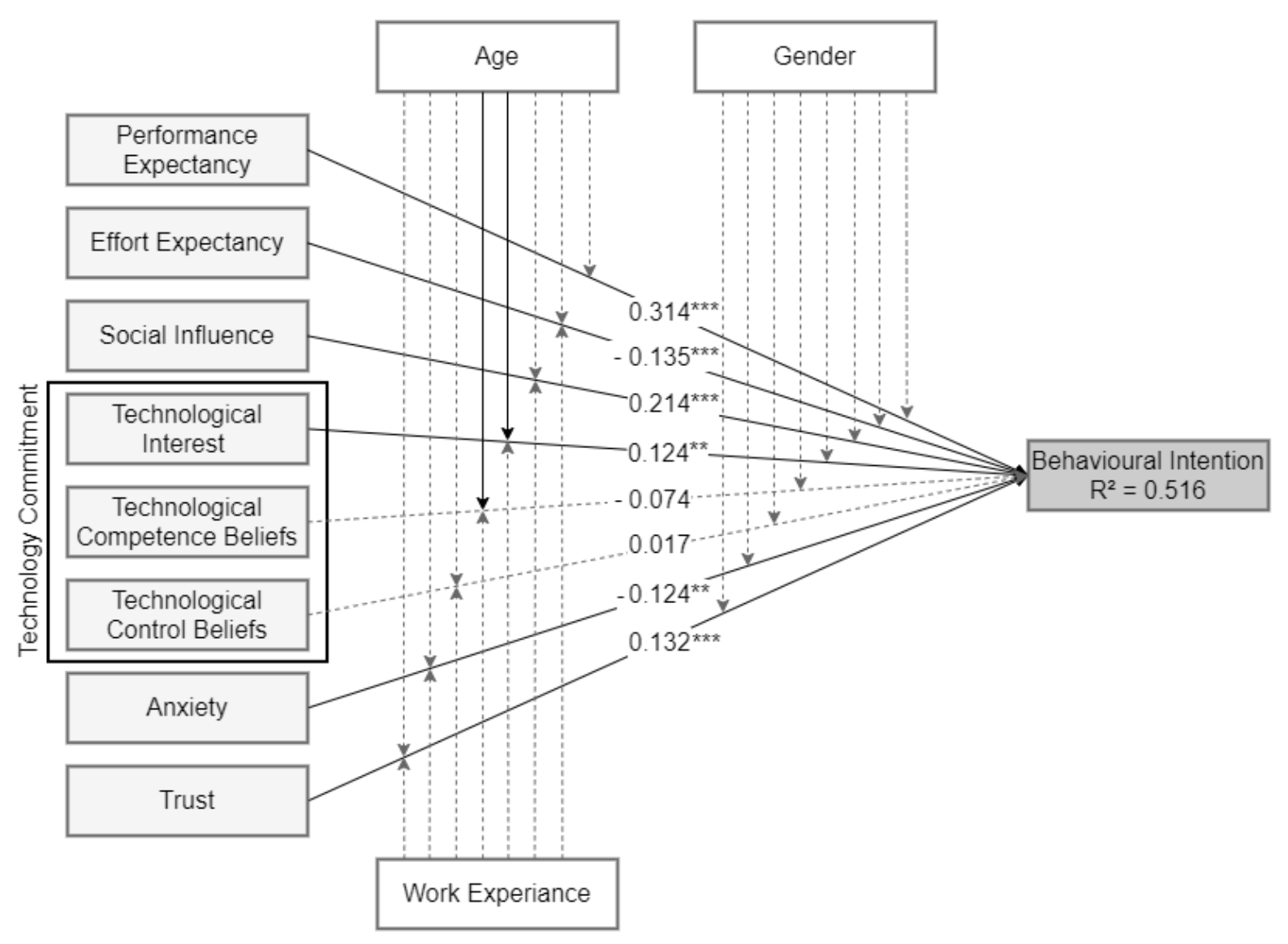

Figure 3: Results of the PLS analysis. Significance level: *p $\leq 0.05, * * p \leq 0.01, * * * p \leq 0.001$; broken line: not significant

Source: Authors' illustration

\section{Discussion}

After analysing the descriptive results (see Table A1 in the appendix) of the survey $(n=500)$ based on the extended UTAUT model, it was found that the respondents are positive about AFR, although the use of AFR on their own farms is not currently planned by the respondents. This was reflected in specific by performance expectancy and the resulting intention of farmers to use AFR. It is hoped that a high degree of technology autonomy will reduce farmers' workload, which is in line with the results of RIAL-LOVERA (2018), who analysed drivers, barriers, and opportunities for adoption of agricultural robotics in California (USA) by applying qualitative expert interviews. The positive behavioural intention underlines the respondents' willingness to use AFR. This is confirmed by the indefiniteness regarding effort expectancy, the lack of anxiety regarding AFR and the respondents' high technology commitment. Especially the latter is important for the future adoption of AFR. For example, AUSTIN et al. (1998) have already shown that farmers are 
more likely to use new production methods if they have a high level of technological interest; even more so if they want to adopt a technology as a pioneer (Voss et al., 2008).

Furthermore, the PLS analysis showed that the six constructs performance expectancy, effort expectancy, social influence, technological interest, anxiety, and trust significantly determine farmers' behavioural intention to use AFR and that the assumed research model has a relatively high explanatory power $\left(\mathrm{R}^{2}=51.6 \%\right)$. The fact that the strongest significant positive influence on behavioural intention derives from performance expectancy has been demonstrated in many other studies on technology acceptance (VENKATESH et al., 2003). The significant influence of performance expectancy can also be explained by the relative importance of the economic benefits expected from the use of AFR (REDHEAD et al., 2015). For example, SHOCKLEY et al. (2019) confirmed in their comparison of conventional and autonomous machinery for grain crop production under various scenarios that AFR will never be adopted, if they are not profitable for producers. In their case study, autonomous machinery was more profitable than conventional machinery for all scenarios. In addition, all studies published after 1990 on the economics of AFR that were reviewed by LOWENBERG-DEBOER et al. (2020) figured scenarios in which autonomous robotic technologies are profitable. At the same time, they identified a need for in-depth research on economic implications due to the different study designs, assumptions and calculations only based on data from prototypes.

The significant positive effect of social influence on the behavioural intention to use AFR may result from the fact that farmers are important members of rural communities (RETTER et al., 2002). Therefore, the expectations of third parties are crucial in their decision-making process (SOLANO et al., 2003; ZIMMERMANN, 2003). This supports the concerns expressed by DEVITT (2018) that farmers could fear a social cognition loss due to AFR's onfarm implementation, which, if so, could lead to AFR possibly not reaching the post-adoption phase of acceptance and use. Various studies have shown that farmers perceive society's increasingly negative perception of their production methods as a burden (ZANDER et al., 2013). In contrast to this a consumer survey carried out in EU Member States in 2012 found that only $6 \%$ of respondents would agree to a ban on robots in agriculture (EUROBAROMETER, 2012). If AFR lead to wider consumer acceptance than current agricultural production methods, this could be an additional incentive for farmers to use AFR.

Regarding technology commitment, only the facet technological interest had a significant influence on the behavioural intention to use AFR. The intention to use new technologies often requires a certain affinity for or interest in technology (AUSTIN et al., 1998; Voss et al., 2008). For the behavioural intention to use a new technology, trust is one of the most 
important predictors (GEFEN et al., 2003). The knowledge about the reliability of a system determines how to use it (DIXON et al., 2007; DIXON and WICKENS, 2006; MEYER, 2001, MEYER, 2004). Since trust had a positive influence on the behavioural intention, it can be said that safety and performance issues are important in relation to AFR. Nevertheless, it must be assumed that most farmers are unlikely to be aware of the detailed requirements that their farm must meet in order to fulfil AFR's needs or whether the technology works reliably and is therefore trustworthy (REDHEAD et al., 2015). Similarly, the conclusion reached by DEVITT (2018) that a lack of trust could have an inhibiting effect on the behavioural intention to use AFR could be confirmed indirectly.

The significant negative influence of effort expectance on the intention to use AFR was to be expected, since decision-making in agriculture traditionally tends to avoid risk rather than increase efficiency (TEY and BRINDAL, 2012). According to REDHEAD et al. (2015), Australian farmers fear the level of complexity of AFR, the lack of employees skilled in operation of this new technology and limited access to reliable economical communication and data storage service. This could also be an issue for German farmers, as they work in a developed country as well. At the same time, anxiety, as the counterpart of trust, could also be validated by its significantly negative influence on the behavioural intention to use AFR, although the positive effect of trust was greater than the negative effect of anxiety (HEERINK et al., 2010).

The moderating influence of age was demonstrated for the relation between the behavioural intention and the two facets technological interest and technological competence beliefs of the technology commitment construct. Younger farmers showed a higher level of technological interest and believe that they have more skills in handling new technology than older farmers. These results are in line with many other studies analysing the effect of age and technological interest on new technologies in agriculture (ISGIN et al., 2008; PIERPAOLI et al., 2013; TEY and BRINDAL, 2012), as younger farmers are more likely to adopt new agricultural technologies (LARSON et al., 2008).

Work experience in no case moderates the relationships between the independent and dependent variables in the extended UTAUT model. However, former studies showed that experience plays an important role for the acceptance of new technologies or systems in agriculture (HIEBERT, 1974; SCHUKAT et al., 2019). It remains to be considered whether a median split regarding MGA draws a suitable dividing line for age and work experience, since, for example, farmers with more than 10 years of work experience could also be considered as experienced. 
However, this study finds its limitations in several biases which cannot be excluded due to the way the data is collected. Particularly when it comes to the self-assessment of respondents with regard to certain beliefs, phenomena such as response behaviour not corresponding to reality as a result of social desirability, a tendency towards the middle (BORTZ and DÖRING, 2006) or simple incomprehensibility of the question asked can always occur. In addition, online surveys often tend towards self-selection instead of the desired random selection (JACOBS et al., 2009). Thus, opponents or supporters of AFR may feel addressed by the questionnaire and take up a disproportionate share of the sample. Similarly, both age and farm size structure of the investigated sample is younger and larger than German average, so that the result may indicate a more technophile attitude of respondents compared to the German average. Nevertheless, since AFR will only be relevant for future operational decisions, a younger sample composition can be assumed to be more relevant. For the MGA, the sample is divided into two groups, which leads to a high degree of data aggregation and a loss of information (ORCUTT et al., 1968). Thus, if the moderating effects are examined differently, they could possibly be stronger or weaker.

Regarding the path coefficients, it is questionable whether values smaller than 0.1 should be considered for interpretation. Reference values for path coefficients differ in the reviewed literature. Some consider a value smaller than 0.2 to be negligible (CHIN, 1998), while others consider only path values smaller than 0.1 to be negligible (LOHMÖLLER, 1988). The path coefficients of technological interest (0.124) and anxiety (-0.124) were only slightly above an absolute value of 0.1 and therefore had only a small influence but were nonetheless considered in the discussion. Since AFR is a future technology in Germany, it was only possible to determine the behavioural intention to use AFR. But a gap is often observed between the intention to use and the actual usage behaviour (RENNER et al., 2007), so that farmers may behave differently than ex-ante intended. The results should therefore be considered cautiously. However, despite the limitations mentioned, the model can explain more than $50 \%$ of the variance. Therefore, it seems suitable to predict the behavioural intention of farmers towards AFR.

It is worth noting that the added constructs trust and anxiety had significant effects on the behavioural intention to use AFR. Thus, the study could again prove the adaptability of the UTAUT and furthermore its transferability to the context of autonomous agritechnical concepts, which is of interest for future technology acceptance studies using the UTAUT model.

The findings provide several implications for agricultural manufacturers and politicians. The present results show a broadly positive position within the agricultural sector in favour 
of this new technology, although this should be viewed with some caution as the study is not entirely representative in its composition. The absence of anxiety in relation to AFR and the high level of technological commitment are further evidence for this. AUSTIN et al. (1998) have already shown that farmers are more likely to use new production methods if they have a high level of technological interest; even more if they want to adopt a new technology as a pioneer (Voss et al., 2008). Manufacturers should build on this and continue to increase the performance expectations of farmers, for example by demonstrating the economic attractiveness of AFR compared with conventional agricultural machinery in certain fields. In case of Australia farmers welcomed the opportunity to be involved in the prototype development of AFR, which could also be an option for Germany (REDHEAD et al., 2015). The ecological benefits attributed to AFR could meet public expectations of farming and should be better communicated to address the social influence on the behavioural intention to use AFR (FRIEDRICH et al., 2012). In addition to better communication of the benefits, specific incentives, such as training on AFR, could be provided to reduce effort expectations and anxiety. DEVITT (2018) sees this as one of the greatest barriers to the adoption of AFR. There are various ways in which policymakers can promote the use of AFR or reduce the risks involved (GERYBADZE, 2015). For example, a supply-side promotion in sense of a technology push or a demand-side promotion in sense of a market pull would be possible. In addition, politicians could try to create better framework conditions, for example by establishing a stable legal situation or financial support programmes for autonomous systems. Another approach could target society or the social environment of farmers, for example by proactively providing information or education.

\section{Conclusions}

AFR are considered to be a promising technology for addressing many agricultural production challenges as well as a growing conflict in Western Europe between societal expectations regarding ecological sustainability and modern farming practices (MINBEN et al., 2015). However, this promising technology will only be established on a broad basis if it is accepted by its potential future users (REDHEAD et al., 2015; VENKATESH et al., 2003). The technology acceptance is determined by various parameters, such as the economic feasibility of the technology, national policies and the legal situation resulting from these policies. For a new technology, like AFR, the acceptance among farmers is expressed essentially by the behavioural intention to use the technology, which has been partially supported by studies of technologies that are part way through the adoption process (e.g., AMBOng and PAulino, 2020; AMIN and Li, 2014; FAR and RAZAEI- 
MoghadDAM, 2015; SchuKAT et al., 2019). Therefore, the aim of this study was a first ex-ante quantification of the determinants influencing the behavioural intention of farmers to use AFR. For this purpose, a research model was developed based on the UTAUT proposed by VENKATESH et al. (2003).

The results offer a range of opportunities for further studies. For example, the results found must be confirmed or rejected by a representative sample. In addition, discrete choice experiments could be used to aggregate the collected preferences into more robust results. Finally, comparative follow-up studies should survey the actual adoption behaviour of farmers as soon as AFR have been introduced to the market on a broader scale and initial experience with this new technology is available. Although a fundamentally positive view of farmers in Germany towards AFR could be demonstrated, a differentiated opinion of other stakeholder groups, such as the established agricultural machinery manufacturers or AgTech startups, has not yet been researched in Germany. Due to the high level of public interest in agricultural production methods (ZANDER et al., 2013; ZÜHLSDORF et al., 2016), it would also be interesting to survey public attitudes towards AFR. If there is too much resentment against AFR, this could hinder the introduction of this new technology (FERRETTI and PAVONE, 2009).

\section{References}

Ambong, R. M. A. and M. A. Paulino (2020): Analyzing Rice Farmers' Intention to Adopt Modern Rice Technologies Using Technology Acceptance Model (TAM). In: Asian Research Journal of Agriculture 13 (1): 21-30, doi: 10.9734/arja/2020/v13i130094.

Ajzen, I. (1991): The theory of planned behavior. In: Organizational Behavior and Human Decision Processes 50 (2): 179-211, doi: 10.1016/0749-5978(91)90020-T.

AJZEN, I. (2005): Attitudes, Personality and Behavior. Open University Press, Berkshire.

Austin, E. J., Willock, J., Deary, I. J., Gibson, G. J., Dent, J. B., Edwards-Jones, G., Morgan, O., GRIEVE, R. and A. SUTHERLAND (1998): Empirical models of farmer behaviour using psychological, social and economic variables. Part I: linear modelling. In: Agricultural Systems 58 (2): 203-224, doi: 10.1016/S0308-521X(98)00066-3.

BARON, R. and D. KENNY (1986): The moderator-mediator variable distinction in social psychological research: Conceptual, strategic, and statistical considerations. In: Journal of Personality and Social Psychology 51 (6): 1173-1182, doi: 10.1037//0022-3514.51.6.1173.

Basu, S., Omotubora, A., Beeson, M. and C. Fox (2020): Legal framework for small autonomous agricultural robots. In: AI \& Society 35: 113-134, doi: 10.1007/s00146-018-0846-4.

BEIER, G. (1999): Kontrollüberzeugungen im Umgang mit Technik. In: Report Psychologie 24 (9): 684-693.

BEM, D. J. and A. ALLEN (1974): On predicting some of the people some of the time: The search for crosssituational consistencies in behavior. In: Psychological Review 81 (6): 506-520, doi: 10.1037/h0037130.

BORTZ, J. and N. DÖRING (2006): Forschungsmethoden und Evaluation für Human- und Sozialwissenschaftler. Springer, Berlin/Heidelberg. 
BozIOnElos, N. (1996): Psychology of computer use: XXXIX. Prevalence of computer anxiety in British managers and professionals. In: Psychological reports 78 (3): 995-1002, doi: 10.2466/pr0.1996.78.3.995.

BRAUER, R. R. (2017): Akzeptanz kooperativer Roboter im industriellen Kontext. Universitätsverlag Chemnitz, Chemnitz.

Brehm, J. W. (1966): A theory of psychological reactance. Academic Press, New York.

Chikoye, D. M., GuPTA, N. K. and K. R. KANDADI (2018): Application of UTAT in understanding the adoption of technologies for reducing post harvest maize in Zambia. In: International Journal of Agriculture and Environmental Research 4 (3): 610-636.

CHIN, W. (1998): The Partial Least Squares Approach to Structural Equation Modeling. In: G. MARCOULIDES (Ed.): Methodology for Business and Management - Modern Methods for Business Research. Lawrence Erlbaum Associates Publishers, Mahwah: 295-336.

CoHEN, J. (1988): Statistical power analysis for the behavioral sciences. Lawrence Erlbaum Associates Publishers, Mahwah.

D'AnTONi, J. M., MishrA, A. K. and H. JOO (2012): Farmers' perception of precision technology: The case of autosteer adoption by cotton farmers. In: Computers and Electronics in Agriculture 87: 121-128, doi: 10.1016/j.compag.2012.05.017.

DAVIS, F. D. (1989): Perceived Usefulness, Perceived Ease of Use, and User Acceptance of Information Technology. In: MIS Quarterly 13 (3): 319-340, doi: 10.2307/249008.

Davison, A. C. and D. V. Hinkley (1997): Bootstrap Methods and their Application. Cambridge University Press, Cambridge.

Destatis (2019): Landwirtschaftliche Betriebe. Betriebsgrößenstruktur landwirtschaftlicher Betriebe nach Bundesländern (Agricultural enterprises. Size structure of agricultural enterprises by federal states). URL: https://www.destatis.de/DE/Themen/Branchen-Unternehmen/Landwirtschaft-ForstwirtschaftFischerei/Landwirtschaftliche-Betriebe/Tabellen/betriebsgroessenstruktur-landwirtschaftlichebetriebe.html (accessed 2 January 2020).

DevitT, S. K. (2018): Cognitive Factors that Affect the Adoption of Autonomous Agriculture. In: Farm Policy Journal 15 (2): 49-60.

Diamantopoulos, A. (2011): Incorporating Formative Measures into Covariance-Based Structural Equation Models. In: MIS Quarterly 35 (2): 335-358, doi: 10.2307/23044046.

DiXON, S. and C. WiCKENS (2006): Automation Reliability in Unmanned Aerial Vehicle Control: A RelianceCompliance Model of Automation Dependence in High Workload. In: Human factors 48 (3): 474-486, doi: 10.1518/001872006778606822.

DiXon, S., WiCkens, C. and J. MCCARLEY (2007): On the Independence of Compliance and Reliance: Are Automation False Alarms Worse Than Misses? In: Human factors 49 (4): 564-572, doi: 10.1518/001872007X215656.

Efron, B. and R. Tibshirani (1986): Bootstrap Methods for Standard Errors, Confidence Intervals, and Other Measures of Statistical Accuracy. In: Statistical Science 1 (1): 54-75.

Eurobarometer (2012): Public Attitudes towards Robots. Special Eurobarometer 382. URL: https://ec.europa.eu/commfrontoffice/publicopinion/index.cfm/Survey/getSurveyDetail/instrument s/SPECIAL/surveyKy/1044/p/3 (accessed 10 November 2019).

EUROSTAT (2019): Labour force main indicators. URL: https://appsso.eurostat.ec.europa.eu/nui/submitViewTableAction.do (accessed 29 August 2019).

FAR, S. T. and K. REZAEI-MOgHADDAM (2015): Determinants of Iranian Agricultural Consultants' Intentions toward Precision Agriculture: Integrating Innovativeness to the Technology Acceptance Model. In: Journal of the Saudi Society of Agricultural Sciences 16 (3): 280-286, doi: 10.1016/j.jssas.2015.09.003. 
Ferretti, M. P. and V. PAVONE (2009): What do civil society organisations expect from participation in science? Lessons from Germany and Spain on the issue of GMOs. In: Science \& Public Policy 36 (4): 287-299, doi: 10.3152/030234209X436527.

FORNELL, C. and D. F. LARCKER (1981): Evaluating structural equation models with unobservable variables and measurement error. In: Journal of Marketing Research 18 (1): 39-50, doi: 10.2307/3151312.

Foster, A. D. and M. R. RosenzWEIG (1995): Learning by Doing and Learning from Others: Human Capital and Technical Change in Agriculture. In: Journal of Political Economy 103 (6): 1176-1209, doi: $10.1086 / 601447$.

Friedrich, T., DERPSCH, R. and A. KASSAM (2012): Overview of the Global Spread of Conservation Agriculture. In: Field Actions Science Report 6 (1): 1-7, doi: 10.1080/00207233.2018.1494927.

Gallimore, D., LyOns, J. B., Vo, T., MAHOnEY, S. and K. T. WynnE (2019): Trusting Robocop: GenderBased Effects on Trust of an Autonomous Robot. In: Frontiers in Psychology 10 (2019): 482, doi: 10.3389/fpsyg.2019.00482.

Gefen, D., Karahanna, E. and D. Straub (2003): Inexperience and Experienced with Online Stores: The Importance of TAM and Trust. In: IEEE Transactions on Engineering Management 50 (3): 307-321.

GERMAN FARMERS’ ASSOCIATION (2019). Situationsbericht 2019/20. Trends und Fakten zur Landwirtschaft. DBV, Berlin.

GERYBADZE, A. (2015): Instrumente der Innovationspolitik. Auf dem Weg zu einer neuen Industriepolitik? In: WSI-Mitteilungen 68: 516-525, doi: 10.5771/0342-300X-2015-7-516.

GHAZAli, A. S., HAM, J., BArAKOva, E. and P. MARKOPOUlos (2018): Effects of Robot Facial Characteristics and Gender in Persuasive Human-Robot Interaction. In: Frontiers in Robotics and AI 5: 1-16, doi: 10.3389/frobt.2018.00073.

GLOY, B. A. and J. AKRIDGE (2000): Computer and Internet adoption on large U.S. farms. In: The International Food and Agribusiness Management Review 3 (3): 323-338, doi: 10.1016/S1096-7508(01)00051-9.

GÖTZ, O., LIEHR-GOBBERS, K. and M. KRAFFT (2010): Evaluation of Structural Equation Models Using the Partial Least Squares (PLS) Approach. In: EsPosito VinZI, V., CHIN, W. W., Henseler, J. and H. WANG (Eds.): Handbook of Partial Least Squares: Concepts, Methods and Applications. Springer, Berlin/Heidelberg: 691-711.

Hair, J. F., Hult, G., Ringle, C. and M. SARStedt (2016): A Primer on Partial Least Squares Structural Equation Modeling. Sage Publications, Thousand Oaks.

HAIR, J. F., RINGLE, C. and M. SARSTEDT (2011): PLS-SEM: Indeed a silver bullet. In: The Journal of Marketing Theory and Practice 19: 139-151, doi: 10.2753/MTP1069-6679190202.

Heerink, M., Krose, B., Evers, V. and B. WielingA (2010): Assessing Acceptance of Assistive Social Agent Technology by Older Adults: the Almere Model. In: International Journal of Social Robotics 2 (4): 361 375, doi: 10.1007/s12369-010-0068-5.

HENSELER, J. (2005): Einführung in die PLS-Pfadmodellierung. In: WiSt - Wirtschaftswissenschaftliches Studium 34 (2): 70-75, doi: 10.15358/0340-1650-2005-2-70.

Henseler, J. and W. W. CHIN (2010): A Comparison of Approaches for the Analysis of Interaction Effects Between Latent Variables Using Partial Least Squares Path Modeling. In: Structural Equation Modeling: A Multidisciplinary Journal 17 (1): 82-109, doi: 10.1080/10705510903439003.

Henseler, J., Ringle, C. and M. SARstedt (2015): A New Criterion for Assessing Discriminant Validity in Variance-based Structural Equation Modeling. In: Journal of the Academy of Marketing Science 43 (1): 115-135, doi: 10.1007/s11747-014-0403-8.

Henseler, J., Ringle, C. M. and R. R. SinkOvics (2009): The use of partial least squares path modeling in international marketing. In: Rudolf, R. S. and N. G. PERVEZ (Eds.): New Challenges to International Marketing. Emerald Group Publishing Limited, Bingley: 277-319.

HertzBERG, J. (2014): Autonome Systeme in der Landwirtschaft. KTBL, Darmstadt. 
HieBERT, L. D. (1974): Risk, Learning, and the Adoption of Fertilizer Responsive Seed Varieties. In: American Journal of Agricultural Economics 56 (4): 764-768, doi: 10.2307/1239305.

HoAg, D. L., Ascough, J. C. and W. M. Frasier (1999): Farm Computer Adoption in the Great Plains. In: Journal of Agricultural and Applied Economics 31 (1): 57-67, doi: 10.1017/S0081305200028776.

Hornburg, C. and A. GIERING (1996): Konzeptualisierung und Operationalisierung komplexer Konstrukte. Ein Leitfaden für die Marketingforschung. In: Marketing ZFP 18 (1): 5-24, doi: 10.15358/0344-13691996-1-5.

HULLAND, J. (1999): Use of partial least squares (PLS) in strategic management research: a review of four recent studies. In: Strategic Management Journal 20 (2): 195-204, doi: 10.1002/(SICI)10970266(199902)20:2<195::AID-SMJ13>3.0.CO;2-7.

IsGin, T., BILGIC, A., Forster, D. L. and M. T. BATTE (2008): Using count data models to determine the factors affecting farmers' quantity decisions of precision farming technology adoption. In: Computers and Electronics in Agriculture 62 (2): 231-242, doi: 10.1016/j.compag.2008.01.004.

Jacobs, B., Hartog, J. and W. Vijverberg (2009): Self-selection Bias in Estimated Wage Premiums for Earnings Risk. Empirical Economics 37 (2): 271-286, doi: 10.1007/s00181-008-0231-0.

Kester, C., Griepentrog, H. W., Hörner, R. and Z. TunCER (2013): A survey of future farm automation - a descriptive analysis of survey responses. In: J. V. STAFFORD (Ed.): Precision Agriculture' 13. Wageningen Academic Publishers, Wageningen: 785-792, doi:10.3920/978-90-8686-778-3_97.

AMIN, K. and J. LI (2014): Applying farmer technology acceptance model to understand farmer's behavior intention to use ICT based microfinance platform: A comparative analysis between Bangladesh and China. Wuhan International Conference on e-Business (WHICEB) Proceedings at the AIS Electronic Library (AISeL), Wuhan, China.

KING, A. (2017): Technology: The Future of Agriculture. Nature 544 (7651): 21-23, doi: 10.1038/544S21a.

KRAMPEN, G. (1991): Fragebogen zu Kompetenz- und Kontrollüberzeugungen: (FKK); Handanweisung. Hogrefe Verlag, Göttingen.

Krampen, G., Viebig, J. and W. WAlter (1982): Entwicklung einer Skala zur Erfassung dreier Aspekte von sozialem Vertrauen. In: Diagnostica 28 (3): 242-247.

KUCZERA, C. (2006): Der Einfluss des sozialen Umfeldes auf betriebliche Entscheidungen von Landwirten. Margraf Publishers, Weikersheim.

Kulviwat, S., Bruner II, G. C., Kumar, A., NAsco, S. A. and T. ClarK (2007): Toward a unified theory of consumer acceptance technology. In: Psychology \& Marketing 24 (12), 1059-1084, doi: 10.1002/mar.20196.

KutTer, T., Tiemann, S., SieberT, R. and S. Fountas (2011): The role of communication and co-operation in the adoption of precision farming. In: Precision Agriculture 12 (2011): 2-17, doi: 10.1007/s11119009-9150-0.

Larson, J., Roberts, R. K., English, B. C., Larkin, S. L., Marra, M. C., Martin, S. W., Kenneth, W. P. and J. M. REEVES (2008): Factors affecting farmer adoption of remotely sensed imagery for precision management in cotton production. In: Precision Agriculture 9 (2008): 195-208, doi: 10.1007/s11119008-9065-1.

LEVY, J. A. (1988): Intersections of Gender and Aging. In: The Sociological Quarterly 29 (4): 479-486.

LOHMÖLLER, J.-B. (1988): The PLS Program System: Latent Variables Path Analysis with Partial Least Squares Estimation. In: Multivariate Behavioral Research 23 (1): 125-127, doi: 10.1207/s15327906mbr2301_7.

Lowenberg-DeBoer, J., HuAng, I. Y., Grigoriadis, V. and S. BlackMORE (2020): Economics of robots and automation in field crop production. In: Precision Agriculture 21 (2020): 278-299, doi: 10.1007/s11119-019-09667-5. 
LUTZ, K. J. (2017): Digitalisierung der Landwirtschaft: Revolution mit evolutionärem Charakter. In: HildebrandT, A. and W. LANDhäUBER (Eds.): CSR und Digitalisierung. Der digitale Wandel als Chance und Herausforderung für Wirtschaft und Gesellschaft. Springer Gabler, Berlin: 429-442.

MacMillan, K., Money, K., Downing, S. and C. HillendBrand (2005): Reputation in Relationships: Measuring Experiences, Emotions and Behaviors. In: Corporate Reputation Review 8 (3): 214-232, doi: 10.1057/palgrave.crr.1540251.

MCAllister, D. J. (1995): Affect- and Cognition-Based Trust as Foundations for Interpersonal Cooperation in Organizations. In: The Academy of Management Journal 38 (1): 24-59, doi: 10.2307/256727.

MEYER, J. (2001): Effects of Warning Validity and Proximity on Responses to Warnings. In: Human factors 43 (4): 563-572, doi: 10.1518/001872001775870395.

MEYER, J. (2004): Conceptual Issues in the Study of Dynamic Hazard Warnings. In: Human factors 46 (2): 196204, doi: 10.1518/hfes.46.2.196.37335.

Minßen, T.-F., UrSO, L.-M., GAus, C.-C. and L. Frerichs (2015): Mit autonomen Landmaschinen zu neuen Pflanzenbausystemen. In: ATZoffhighway 8 (3): 6-11, doi: 10.1007/s35746-015-0028-y.

Minton, H. L. and F. W. SCHNEIDER (1980): Differential psychology. Waveland Press, Long Grove.

Morris, M. G. and V. VenKatesh (2000): Age Differences in Technology Adoption Decisions: Implications for a Changing Workforce. In: Personnel Psychology 53 (2): 375-403, doi: 10.1111/j.17446570.2000.tb00206.x.

MubHoff, O., Hirschauer, N. and H. WAssmuss (2009): The Role of Bounded Rationality in Farm Financing Decisions - First Empirical Evidence -. Paper presented at the International Association of Agricultural Economists (IAAE) 2009 Conference, Beijing, China.

Neyer, F. J., Felber, J. and C. GebHardt (2012): Entwicklung und Validierung einer Kurzskala zur Erfassung von Technikbereitschaft. In: Diagnostica 58 (2): 87-99, doi: 10.1026/0012-1924/a000067.

Nomura, T., SuzUKi, T., KANDA, T. and K. KATO (2006): Measurement of Anxiety toward Robots. In: ROMAN 2006 - The 15th IEEE International Symposium on Robot and Human Interactive Communication: 372-377, doi: 10.1109/ROMAN.2006.314462.

Orcutt, G. H., Watts, H. W. and J. B. Edwards (1968): Data Aggregation and Information Loss. In: The American Economic Review 58 (4): 773-787.

PICKEL, P. (2019): RoundTable: Agricultural robotics storms global market. Presentation on the International Forum of Agricultural Robotics (FIRA), Toulouse.

Pierpaoli, E., Carli, G., Pignatti, E. and M. Canavari (2013): Drivers of Precision Agriculture Technologies Adoption: A Literature Review. In: Procedia Technology 8 (2013): 61-69, doi: 10.1016/j.protcy.2013.11.010.

Plude, D. and W. Hoyer (1985): Attention and Performance: Identifying and Localizing Age Deficits. In: N. ChARNess (Ed.): Aging and Human Performance. John Wiley and Sons, New York: 47-99.

Redhead, F., SNow, S., Vyas, D., Bawden, O., Russell, R., Perez, T. and M. Brereton (2015): Bringing the Farmer Perspective to Agricultural Robots. In: BEgOLE, B., KIM, J., INKPEN, K. and W. WOO (Eds.): Proceedings of the 33rd Annual ACM Conference Extended Abstracts on Human Factors in Computing Systems. Association for Computing Machinery, New York: 1067-1072, doi: 10.1145/2702613.2732894.

RenNer, B., SpIVAK, Y., KWON, S. and R. SCHWARZER (2007): Does Age Make a Difference? Predicting Physical Activity of South Koreans. In: Psychology and aging 22 (3): 482-493, doi: 10.1037/08827974.22.3.482.

RetTER, C., STAHR, K. and H. BOLAND (2002): Zur Rolle von Landwirten in dörflichen Kommunikationsnetzwerken. In: Berichte über Landwirtschaft 80 (3): 446-467.

RHODES, S. R. (1983): Age-related differences in work attitudes and behavior: A review and conceptual analysis. In: Psychological Bulletin 93 (2): 328-367, doi: 10.1037/0033-2909.93.2.328. 
Rial-LoverA, K. (2018): Agricultural Robots: Drivers, Barriers and Opportunities for Adoption. In: N. Tremblay (Ed.): Proceedings of the 14th International Conference on Precision Agriculture, Montreal, 24-27 June 2018. The International Society of Precision Agriculture, Monticello Illinois.

RÜBCKE VON Veltheim, F., Theuvsen, L. and H. Heise (2019): Akzeptanz autonomer Feldroboter im Ackerbaueinsatz: Status quo und Forschungsbedarf. In: Berichte über Landwirtschaft 97 (3): 1-19, doi: 10.12767/buel.v97i3.248.

SARstedt, M., Henseler, J. and C. M. Ringle (2011): Multigroup Analysis in Partial Least Squares (PLS) Path Modeling: Alternative Methods and Empirical Results. In: MARKO, S., MANFrED, S. and R. T. CHARLES (Eds.): Measurement and Research Methods in International Marketing, Advances in International Marketing Vol. 22. Emerald Group Publishing Limited, Bingley: 195-218, doi: 10.1108/S1474-7979(2011)0000022012.

Schukat, S., Kuhlmann, A. and H. Heise (2019): Fattening Pig Farmers' Intention to Participate in Animal Welfare Programs. In: Animals 9 (12): 1042, doi: 10.3390/ani9121042.

SHOCKLEY, J. M., DiLlon, C. R. and S. A. SHEARER (2019): An economic feasibility assessment of autonomous field machinery in grain crop production. In: Precision Agriculture 20 (2019): 1068-1085, doi: 10.1007/s11119-019-09638-w.

SIMON, H. A. (1956): Rational choice and the structure of the environment. In: Psychological Review 63 (2): 129-138, doi: 10.1037/h0042769.

SOlano, C., LEÓN, H., PÉREZ, E. and M. HerRero (2003): The role of personal information sources on the decision-making process of Costa Rican dairy farmers. In: Agricultural Systems 76 (1): 3-18, doi: 10.1016/S0308-521X(02)00074-4.

Tamirat, T., Pedersen, S. M. and K. Lind (2017): Farm and operator characteristics affecting adoption of precision agriculture in Denmark and Germany. In: Acta Agriculturae Scandinavica, Section B - Soil \& Plant Science 68 (4): 349-357, doi: 10.1080/09064710.2017.1402949.

TEY, Y. S. and M. BRINDAL (2012): Factors influencing the adoption of precision agricultural technologies: a review for policy implications. In: Precision Agriculture 13 (6): 713-730, doi: 10.1007/s11119-012-92736.

TurJa, T., AALTONEN, I., TAIPALE, S. and A. OKSANEN (2020): Robot acceptance model for care (RAM-care): A principled approach to the intention to use care robots. In: Information \& Management 57 (5): 103220, doi: 10.1016/j.im.2019.103220.

Venkatesh, V. and F. D. DAvis (2000): A Theoretical Extension of the Technology Acceptance Model: Four Longitudinal Field Studies. In: Management Science 46 (2): 186-204, doi: 10.1287/mnsc.46.2.186.11926.

Venkatesh, V. and M. G. MORRIS (2000): Why Don't Men Ever Stop to Ask for Directions? Gender, Social Influence, and Their Role in Technology Acceptance and Usage Behavior. In: MIS Quarterly 24 (1): 115139, doi: $10.2307 / 3250981$.

Venkatesh, V., Morris, M. G., Davis, G. B. and F. D. Davis (2003): User Acceptance of Information Technology: Toward a Unified View. In: MIS Quarterly 27 (3): 425-478, doi: 10.2307/30036540.

Venkatesh, V., Thong, J. Y. L., Chan, F. K. Y., Hu, P. J. H. and S. A. Brown (2011): Extending the twostage information systems continuance model - Incorporating UTAUT predictors and the role of context. In: Information Systems Journal 21 (6): 527-555, doi: 10.1111/j.1365-2575.2011.00373.x.

Venkatesh, V., Thong, J. Y. L. and X. Xu (2012): Consumer Acceptance and Use of Information Technology: Extending the Unified Theory of Acceptance and Use of Technology. In: MIS Quarterly 36 (1): 157-178, doi: 10.2307/41410412.

Voss, J., Schaper, C., Spiller, A. and L. Theuvsen (2008): Innovationsverhalten in der deutschen Landwirtschaft - Empirische Ergebnisse am Beispiel der Biogasproduktion. Paper presented at the 48th Annual Conference, Bonn, Germany, 24-26 September 2008. German Association of Agricultural Economists (GEWISOLA), Brunswick, doi: 10.22004/ag.econ.52666. 
Weidner, R., Redlich, T. and J. P. Wulfsberg (2015): Technische Unterstützungssysteme. Springer Vieweg, Wiesbaden.

Zander, K., Isermeyer, F., BÜrgelt, D., Christoph-Schulz, I. B., SAlamon, P. and D. Weible (2013): Erwartungen der Gesellschaft an die Landwirtschaft. Stiftung Westfälische Landwirtschaft, Münster.

ZIMMERMANN, M. (2003): Das Kaufverhalten von Landwirten im Bereich landwirtschaftlicher Investitionsgüter und die Auswirkung auf den Marketing-Mix landtechnischer Unternehmen. Cuvillier Verlag, Göttingen.

ZÜHLSDORF, A., SpIller, A., GAUly, S. and S. KÜHL (2016): Wie wichtig ist Verbrauchern das Thema Tierschutz? Präferenzen, Verantwortlichkeiten, Handlungskompetenzen und Politikoptionen. Zühlsdorf + Partner GbR, Berlin. 


\section{Appendix}

Table A1: Observed items and constructs with quality criteria

\begin{tabular}{llccc}
\hline Item & Statement & Mean & $\begin{array}{c}\text { Standard } \\
\text { deviation }\end{array}$ & $\begin{array}{c}\text { Factor- } \\
\text { loading }\end{array}$ \\
\hline Factor "Performance Expectancy" $(A V E=0.533 ; C R A=0.782 ;$ & \multicolumn{2}{c}{ " } & 0.850; & VIF = 1.487) \\
\hline PE1 & I would use AFR whenever they were to be used. & 4.16 & 0.867 & 0.716 \\
PE2 & AFR could lighten my load in certain operations. & 4.49 & 0.729 & 0.785 \\
PE3 & AFR enables me to save work force. & 4.04 & 1.064 & 0.657 \\
PE4 & With AFR I could operate more efficiently. & 3.92 & 1.002 & 0.795 \\
PE5 & AFR would make farming more environmentally friendly. & 3.87 & 0.945 & 0.687 \\
\hline
\end{tabular}

\begin{tabular}{lllll}
\hline Factor "Effort Expectancy" $(A V E=0.647 ; C R A=0.724 ; C R=0.845 ; V I F=1.633)$ \\
\hline EE1 & I imagine the operation of AFR to be difficult. & 2.87 & 0.962 & 0.770 \\
EE2 & Learning how to handle AFR would be difficult for me. & 2.27 & 0.837 & 0.874 \\
EE3 & A safe handling of AFR would be difficult for me. & 2.14 & 0.850 & 0.763
\end{tabular}

Factor "Social Influence" $(A V E=0.738 ; C R A=0.653 ; C R=0.849 ; V I F=1.363)$

\begin{tabular}{llccc}
\hline SI1 & $\begin{array}{l}\text { I think that other farmers would like to see AFR used on } \\
\text { my farm. }\end{array}$ & 3.25 & 0.938 & 0.812 \\
SI2 & $\begin{array}{l}\text { I think it would make a good impression on society if I } \\
\text { used AFR. }\end{array}$ & 3.47 & 1.064 & 0.904
\end{tabular}

\begin{tabular}{|c|c|c|c|c|}
\hline \multicolumn{5}{|c|}{ Factor "Technological Interest" $(A V E=0.546 ; C R A=0.722 ; C R=0.828 ; V I F=1.521)$} \\
\hline TCI1 & I am very curious about new technical innovations. & 4.42 & 0.732 & 0.740 \\
\hline TCI2 & I am always interested in using the latest technical devices. & 3.54 & 0.923 & 0.762 \\
\hline TCI3 & $\begin{array}{l}{[\mathrm{I}] \text { would (...) use much more frequently technical prod- }} \\
\text { ucts than I currently do. }\end{array}$ & 3.70 & 0.990 & 0.691 \\
\hline TCI4 & I quickly find interest in new technical developments. & 4.00 & 0.896 & 0.760 \\
\hline
\end{tabular}

Factor "Technological Competence Beliefs" $(A V E=0.557 ; C R A=0.737 ; C R=0.834$; $V I F=1.484)$

\begin{tabular}{|c|c|c|c|c|}
\hline TCM1 & Technical innovations mostly overwhelm me. & 2.12 & 0.875 & 0.732 \\
\hline TCM2 & $\begin{array}{l}\text { I find it difficult to deal with new technology - as a rule, I } \\
\text { simply do not know how to do that. }\end{array}$ & 1.76 & 0.802 & 0.777 \\
\hline TCM3 & $\begin{array}{l}\text { When dealing with modern technology, I am often afraid } \\
\text { of failing. }\end{array}$ & 1.69 & 0.789 & 0.741 \\
\hline TCM4 & $\begin{array}{l}\text { I'm afraid I'll be more likely to break down technological } \\
\text { innovations (...). }\end{array}$ & 1.58 & 0.735 & 0.735 \\
\hline
\end{tabular}

Factor "Technological Control Beliefs" $(A V E=0.567 ; C R A=0.659 ; C R=0.794 ; V I F=1.186)$

\begin{tabular}{lllll}
\hline TCN1 & $\begin{array}{l}\text { It is in my hands whether I succeed in using technical in- } \\
\text { novations (...). }\end{array}$ & 4.24 & 0.891 & 0.639 \\
TCN2 & $\begin{array}{l}\text { When I (..) have difficulties with technique, it depends } \\
\text { (...) on me to solve it. }\end{array}$ & & 1.48 & 0.720
\end{tabular}


$\begin{array}{llll}\text { TCN3 What happens when I deal with [technology] is (...) under } & 3.96 & 0.832 & 0.880\end{array}$ my control.

\begin{tabular}{|c|c|c|c|c|}
\hline \multicolumn{5}{|c|}{ Factor "Behavioural Intention" $(A V E=0.568 ; C R A=0.751 ; C R=0.840)$} \\
\hline BI1 & I would use AFR immediately on my farm. & 3.44 & 1.038 & 0.824 \\
\hline BI2 & I plan to use AFR on my farm in the future. & 2.70 & 1.163 & 0.727 \\
\hline $\mathrm{BI} 3$ & $\begin{array}{l}\text { I am sure that I would deal with AFR during the first days } \\
\text { of using on my farm too. }\end{array}$ & 4.53 & 0.697 & 0.722 \\
\hline BI4 & $\begin{array}{l}\text { I would like to familiarize myself with the use of AFR } \\
\text { during the first days of use on my farm. }\end{array}$ & 4.60 & 0.659 & 0.737 \\
\hline \multicolumn{5}{|c|}{ Factor "Trust" ( $A V E=0.749 ; C R A=0.673 ; C R=0.856 ; V I F=1.339)$} \\
\hline TR1 & I would trust AFR to make the right decisions. & 3.40 & 0.910 & 0.910 \\
\hline TR2 & I would follow the instructions given to me by an AFR. & 3.16 & 0.891 & 0.819 \\
\hline \multicolumn{5}{|c|}{ Factor "Anxiety" $(A V E=0.606 ; C R A=0.787 ; C R=0.858 ; V I F=1.611)$} \\
\hline ANX1 & If I were to use AFR, I would be afraid of misusing it. & 2.24 & 0.971 & 0.698 \\
\hline ANX2 & Using AFR, I would be afraid to damage it. & 2.03 & 0.976 & 0.637 \\
\hline ANX3 & I find AFR frightening. & 1.52 & 0.800 & 0.878 \\
\hline ANX4 & I find AFR intimidating. & 1.43 & 0.708 & 0.871 \\
\hline
\end{tabular}

Scale from $5=$ totally agree to $1=$ totally disagree; AVE $=$ Average Variance Extracted;

CRA = Cronbach's Alpha; CR = Composite Reliability; VIF = Inner Variance Inflation Factor

Source: Authors' calculation

Table A2: Discriminant validity Fornell-Larcker criterion

\begin{tabular}{l|rrrrrrrrr}
\hline & \multicolumn{1}{|c}{ BI } & EE & PE & SI & ANX & TR & TCI & TCM & TCN \\
\hline BI & $\mathbf{0 . 7 5 4}$ & & & & & & & & \\
EE & -0.433 & $\mathbf{0 . 8 0 4}$ & & & & & & & \\
PE & 0.576 & -0.284 & $\mathbf{0 . 7 3 0}$ & & & & & & \\
SI & 0.474 & -0.175 & 0.443 & $\mathbf{0 . 8 5 9}$ & & & & & \\
ANX & -0.418 & 0.467 & -0.306 & -0.226 & $\mathbf{0 . 7 7 8}$ & & & & \\
TR & 0.430 & -0.282 & 0.388 & 0.371 & -0.253 & $\mathbf{0 . 8 6 6}$ & & & \\
TCI & 0.370 & -0.358 & 0.287 & 0.217 & -0.230 & 0.157 & $\mathbf{0 . 7 3 9}$ & & \\
TCM & -0.251 & 0.444 & -0.088 & 0.011 & 0.382 & -0.061 & -0.342 & $\mathbf{0 . 7 4 7}$ & \\
TCN & 0.173 & -0.182 & 0.157 & 0.105 & -0.205 & 0.122 & 0.284 & -0.151 & $\mathbf{0 . 7 5 3}$ \\
\hline
\end{tabular}

Source: Authors' calculations (highlighted: square roots of AVE values; not highlighted values: construct correlations) 
Table A3: Discriminant validity HTMT criterion

\begin{tabular}{l|lllllllll}
\hline & BI & EE & PE & SI & ANX & TR & TCI & TCM & TCN \\
\hline BI & & & & & & & & & \\
EE & 0.575 & & & & & & & \\
PE & 0.697 & 0.374 & & & & & & \\
SI & 0.636 & 0.255 & 0.603 & & & & & & \\
ANX & 0.523 & 0.642 & 0.353 & 0.272 & & & & & \\
TR & 0.576 & 0.393 & 0.506 & 0.535 & 0.311 & & & & \\
TCI & 0.482 & 0.491 & 0.374 & 0.311 & 0.307 & 0.225 & & & \\
TCM & 0.337 & 0.609 & 0.122 & 0.062 & 0.554 & 0.079 & 0.474 & & \\
TCN & 0.214 & 0.262 & 0.227 & 0.148 & 0.244 & 0.176 & 0.430 & 0.206 & \\
\hline
\end{tabular}

Source: Authors' calculations

Table A4: Results multigroup analysis - moderating effect of age and work experience between the constructs and the behavioural intention to use AFR

\begin{tabular}{|c|c|c|c|c|c|c|}
\hline \multirow[t]{3}{*}{ Path } & \multicolumn{6}{|c|}{ Path coefficients } \\
\hline & \multicolumn{3}{|c|}{ age } & \multicolumn{3}{|c|}{ work experience } \\
\hline & $<41 \mathrm{y}$ & $>40 y$ & $\Delta$ & $<22 \mathrm{y}$ & $>21 y$ & $\Delta$ \\
\hline $\mathrm{EE} \rightarrow \mathrm{BI}$ & -0.158 & -0.125 & 0.033 & -0.150 & -0.111 & 0.039 \\
\hline $\mathrm{PE} \rightarrow \mathrm{BI}$ & 0.339 & 0.304 & 0.035 & 0.329 & 0.305 & 0.025 \\
\hline $\mathrm{SI} \rightarrow \mathrm{BI}$ & 0.202 & 0.199 & 0.003 & 0.226 & 0.199 & 0.027 \\
\hline $\mathrm{ANX} \rightarrow \mathrm{BI}$ & -0.099 & -0.146 & 0.048 & -0.091 & -0.151 & 0.059 \\
\hline $\mathrm{TR} \rightarrow \mathrm{BI}$ & 0.103 & 0.162 & 0.059 & 0.084 & 0.169 & 0.084 \\
\hline $\mathbf{T C I} \rightarrow \mathbf{B I}$ & 0.016 & 0.220 & $0.204^{*}$ & 0.086 & 0.133 & 0.048 \\
\hline $\mathrm{TCM} \rightarrow \mathrm{BI}$ & -0.178 & 0.013 & $0.190^{*}$ & -0.115 & -0.047 & 0.068 \\
\hline $\mathrm{TCN} \rightarrow \mathrm{BI}$ & 0.011 & -0.037 & 0.047 & 0.017 & 0.020 & 0.003 \\
\hline
\end{tabular}

Significance level: ${ }^{*} \mathrm{p} \leq 0.05$

Source: Authors' calculations 
Table A5: Results multigroup analysis - moderating effect of gender between the constructs and the behavioural intention to use AFR

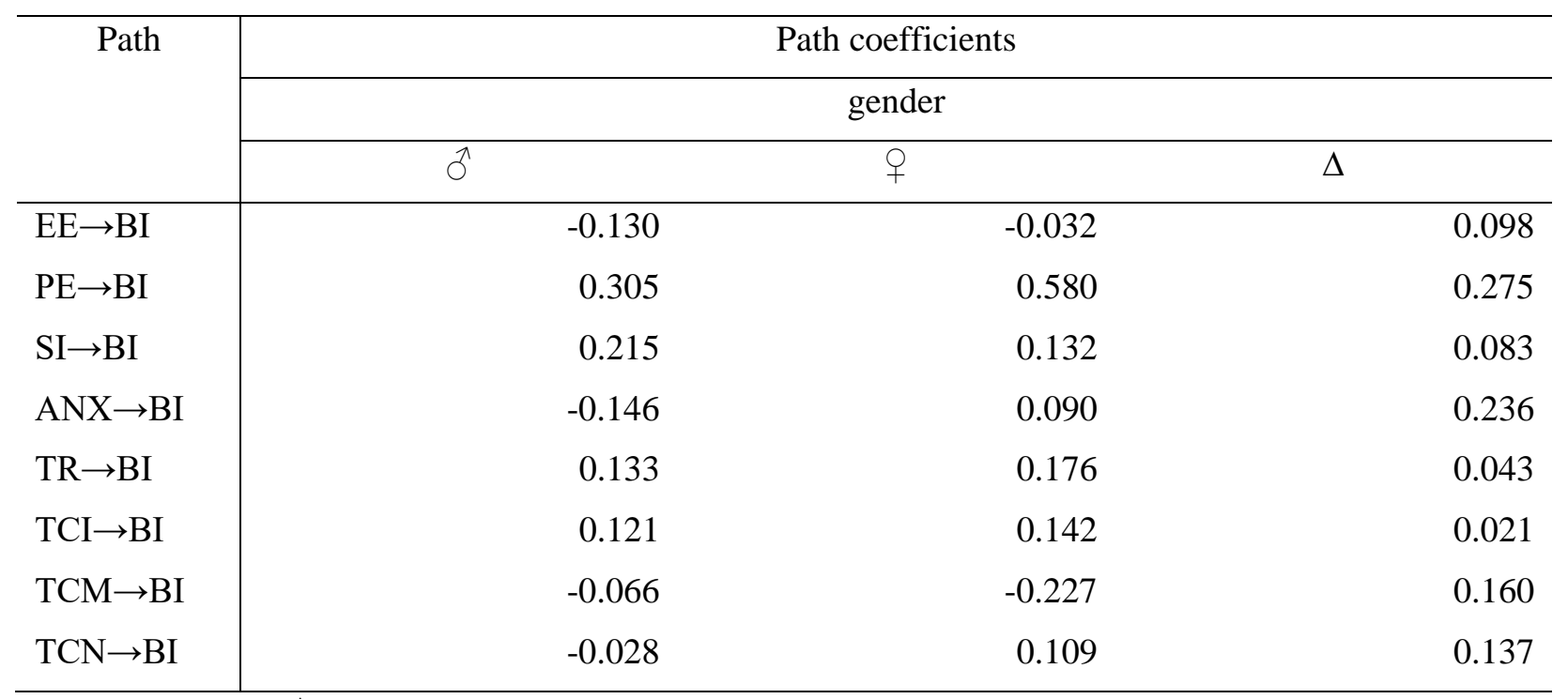

Significance level: ${ }^{*} \mathrm{p} \leq 0.05$

Source: Authors' calculations 


\section{Teil II:}

\section{Einsatz autonomer Feldroboter in der Landwirtschaft aus Sicht von Landmaschinenherstellern}

\section{II.1 Autonomous Field Robots in Agriculture: A Qualitative Analysis of User Acceptance According to Different Agricultural Machinery Companies}

Friedrich Rübcke von Veltheim, Frans Clausen und Heinke Heise

Geleisteter Eigenanteil an dem Beitrag

\begin{tabular}{llllll}
\hline $\begin{array}{l}\text { Literatur- } \\
\text { recherche }\end{array}$ & $\begin{array}{l}\text { Entwicklung } \\
\text { des } \\
\text { Forschungs- } \\
\text { rahmens }\end{array}$ & $\begin{array}{l}\text { Konzept und } \\
\text { Design der Stu- } \\
\text { die }\end{array}$ & $\begin{array}{l}\text { Auswahl, Aus- } \\
\text { führung und } \\
\text { Entwicklung } \\
\text { von Methoden }\end{array}$ & $\begin{array}{l}\text { Interpretation } \\
\text { der } \\
\text { Ergebnisse }\end{array}$ & $\begin{array}{l}\text { Konzept und } \\
\text { Schreiben des } \\
\text { Artikels }\end{array}$ \\
\hline$>60 \%$ & $>75 \%$ & $>60 \%$ & $50 \%$ & $50 \%$ & $>70 \%$ \\
\hline
\end{tabular}

Dieser Beitrag ist so oder in ähnlicher Fassung veröffentlicht in der wissenschaftlichen Zeitschrift , Schriften der Gesellschaft für Wirtschafts- und Sozialwissenschaften des Landbaues e.V. “Bd. 56, 2021. 
II.1 Autonomous Field Robots in Agriculture: A Qualitative Analysis of User Acceptance Accoring to

\section{Inhalt}

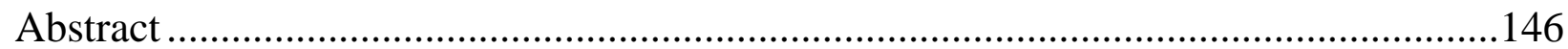

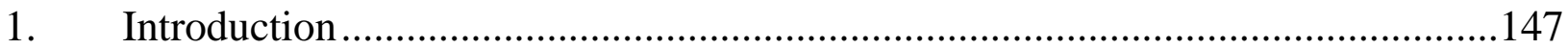

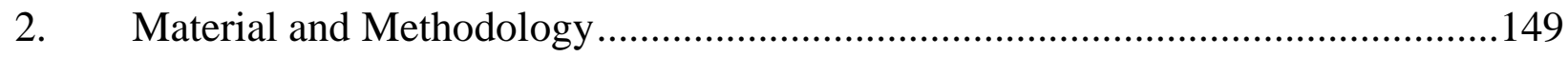

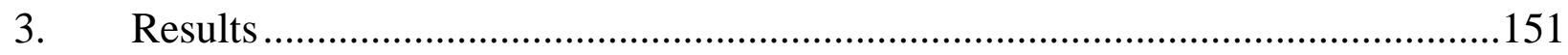

3.1 Sample Description ..................................................................................151

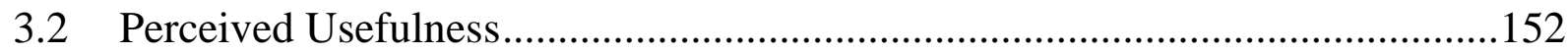

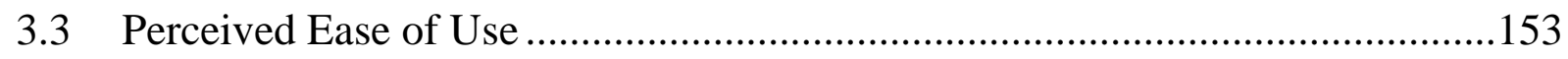

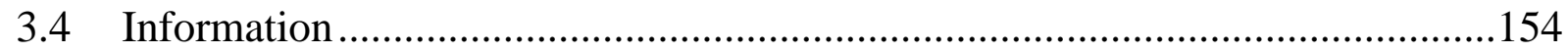

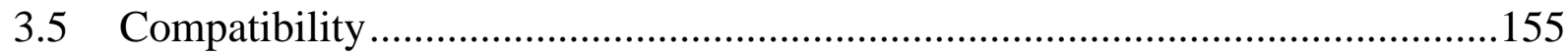

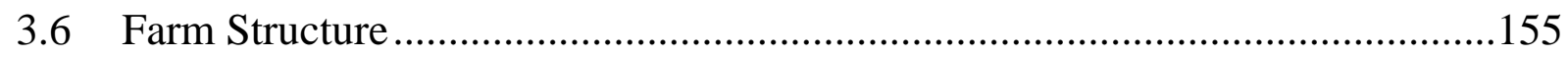

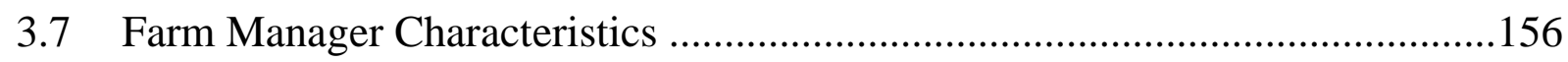

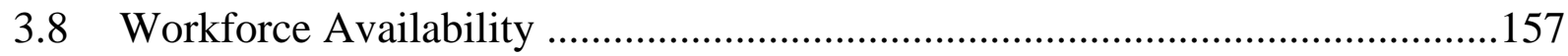

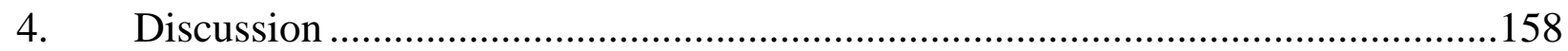

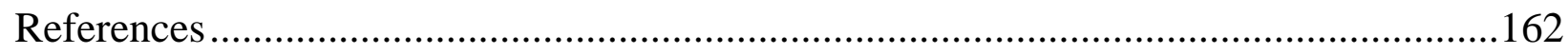




\begin{abstract}
Great hopes are being placed on the autonomation of agricultural engineering from various perspectives. However, it is unclear whether AFR will make the transition from the development phase to the broader agricultural market. Among various factors, this particularly depends on user acceptance of this new technology. Therefore, this paper provides and discusses the results of an initial explorative qualitative survey of the ex-ante user acceptance of AFR from the perspective of nine different agricultural machinery manufacturers in Germany. The interview guideline developed for this purpose is based on an adapted TAM according to DAVIS (1989). In summary, the results confirm that a large number of factors potentially influence farmers' acceptance from the perspective of agricultural machinery manufacturers, with perceived usefulness being regarded as the greatest motivation for using AFR. Even if all experts assume the need for AFR in the future, the economic advantages of autonomous agricultural technology over conventional technology are not entirely plausible over the medium term from the experts' point of view. The interviewees do not expect AFR to be relevant in arable farming for at least 10 years, although earlier for special crops. AFR's future market share will depend above all on legislation regarding autonomous technologies. If AFR are used on a large scale in arable farming, this will very likely lead to changes in existing business models affecting the end customer. If further development of this innovative technology is desired, political decisionmakers should establish a legislative framework for the safe operation of autonomous agricultural machinery and promote the use of AFR through market policy instruments. In addition, the results require quantitative verification among farmers.
\end{abstract}

\title{
Keywords
}

Acceptance, Autonomous, Farmers, Manufacturers, Robots, TAM 
II.1 Autonomous Field Robots in Agriculture: A Qualitative Analysis of User Acceptance According to

\section{Introduction}

Autonomous vehicles are becoming increasingly important not only for the automotive industry but also for agricultural engineering in industrialized countries. A higher degree of automation in agricultural processes enables entire systems to be reconsidered. For example, instead of ever larger and heavier conventional agricultural machinery, small autonomous robot units could replace them, performing certain tasks either alone or in swarms with other units. Whereas in the past smallholder farms were labeled uneconomic, AFR could make them economically attractive again and lead to a shift in thinking, saying: "bigger is no longer better" (KING, 2017). In addition to improved scalability, AFR have the potential to be more environmentally friendly and at the same time economically efficient. For example, due to their lower weight, they exert less soil compaction than large agricultural machinery and their advanced sensor technology enables more precise, plantspecific pest detection and elimination, which can save considerable amounts of pesticides (REDHEAD et al., 2015). By replacing the operator, the math is changed, which can be particularly useful in labor-intensive areas of arable farming, such as special crop cultivation. Due to the machines' usual electric drives, less air and noise pollution also occur (GAUS et al., 2017). This is particularly important given that farmers are influenced by their social environment. For example, KUCZERA (2006) found that colleagues, friends and family members have an influence on the farmer's strategic decisions.

Besides the many advantages, there are also potential disadvantages involved with AFR. The shift from manned tractors to unmanned robot systems with no direct visual contact could make some farmers skeptical. According to REDHEAD et al. (2015), farmers fear highly complex AFR for which they do not have qualified staff. DEVITT (2018) adds further cognitive acceptance barriers amongst farmers, such as a lack of trust in AFR or the ongoing loss of agricultural skills associated with further loss of the already diminished public recognition of farming as a profession. In case of high-value crops, KESTER et al. (2013) revealed a conflict between the support for autonomous technologies on the one hand and general skepticism towards new technology on the other.

However, there is widespread agreement that AFR will find a place in future agriculture. But it is not yet clear, when and to what extent this will happen. KING (2017), for example, quotes the assumption of an AFR manufacturer saying that established agricultural machinery companies have little interest in introducing AFR because they represent alternative designs to their existing business models. On the other hand, this new technology also entails a range of new business models for agricultural machinery manufacturers. One 
II.1 Autonomous Field Robots in Agriculture: A Qualitative Analysis of User Acceptance According to

option could be AFR offered to farmers as a service by an external supplier or as a cloudbased pay-per-use model (ERL et al., 2019). Moreover, the legal size and weight standards for agricultural machinery have been largely exhausted, so that a rethink in terms of smaller, more specific machines offers the opportunity to further develop agricultural technology (GAUS et al., 2017). FUCHS (2018) even assigns AFR a potential disrupter that could represent a caesura in agricultural engineering. In any case, new possibilities for analyzing data collected by AFR will lead to increased competitive pressure among agricultural machinery manufacturers (PHAM and STACK, 2017). In addition to AgTech startups, IT companies such as IBM and Google are entering the agricultural machinery market (BURWOOD-TAYLOR et al., 2016). The current market leaders should be aware of risk to their present position. It is therefore important to assess whether there is a future market for this new technology. The perspective of various agricultural machinery manufacturers on the factors influencing the ex-ante acceptance of AFR among farmers therefore seems particularly interesting.

Nevertheless, only few studies have so far investigated AFR's acceptance process in the agricultural sector. RIAL-LOVERA (2018) interviewed 14 agricultural stakeholders in California about their AFR adoption behavior. The main drivers for adoption were identified as shortages in the workforce and rising labor costs, as well as a missing awareness of the potential benefits of AFR and the lack of compatibility between farm equipment. More broadly, CAFFARO and CAVALLO (2019) interviewed several people about SFT during an agricultural fair in Northern Italy. They found that farm manager characteristics, such as educational level, moderate SFT adoption behavior. In summary, and according to RIALLOVERA (2018), there is an enormous research deficit in the study of AFR's technology acceptance process in agriculture. Many studies focus on the effect of individual aspects and neglect the multidimensionality of the acceptance process, resulting in insufficient knowledge of the complexity of the acceptance factors (BUKCHIN and KERRET, 2018; PATHAK et al., 2019).

To address this under-researched issue, the present study examines the factors influencing the farmers' ex-ante acceptance of AFR from an agricultural machinery manufacturer perspective. Therefore, nine agricultural machinery manufacturers were interviewed on this topic using qualitative expert interviews. 
II.1 Autonomous Field Robots in Agriculture: A Qualitative Analysis of User Acceptance According to

\section{Material and Methodology}

In order to collect the required data, expert interviews in line with MAYRING (2015) were performed with nine agricultural engineering companies. Among these are seven established agricultural equipment and vehicle manufacturers and two AgTech startup companies that produce AFR. The questions were formulated in an open and neutral manner then divided into six thematic blocks: drivers and barriers, technology, economics, socio-economic effects, environmental impacts and legal restrictions. After a brief introduction to the topic, the experts were interviewed personally. Finally, the experts had the opportunity to add to or address issues that were not part of the questionnaire. The interview-guide was divided in 25 questions based on factors influencing ex-ante user acceptance of new technologies according to DAVIS' (1989) TAM, including perceived usefulness, perceived ease of use and external factors. The factors attitude toward using and actual system use were excluded from this analysis deliberately. This was due a direct influence of perceived usefulness and perceived ease of use on the behavioral intention being found (CHUTTUR, 2009). In addition, the study's object concerns the future use of a technology, so very little or no experience in handling AFR can be relied on. DAVIS (1989) defines perceived usefulness as: the subjective probability of a potential user employing a particular technology, in this case AFR, will increase his work performance in an organizational context. Perceived ease of use describes the degree to which the future user expects the technology to be free of any effort. Perceived usefulness and ease of use are statistically distinct dimensions and can be affected by various external factors (BENBASAT and DEXTER, 1986; MILLER, 1977). It is assumed that perceived ease of use has a positive influence on perceived usefulness and on behavioral intention, as ease of use makes a technology appear more useful and increases the probability of use (ADRIAN et al., 2005; Rial-Lovera, 2018). Based on the available literature on this topic, the external factors of information, compatibility, farm structure, farm manager characteristics and workforce availability were added (AUBERT et al., 2012; CAFFARO and CAVAllo, 2019; DeVitT, 2018; KeSTER et al., 2013; PATHAK et al., 2019; PAUSTIAN and THEUVSEN, 2017; REDHEAD et al., 2015; RIAL-LOVERA, 2018). The factor information follows an approach from precision agriculture research and is defined as the information collected by AFR regarding availability and quality as a source for strategic decisions on farms. It is assumed that this factor affects perceived usefulness. At the same time, the possible advantage of additional data over conventional production methods is examined. With the factor compatibility, a feature from diffusion theory is included, which has proven to be an essential aspect in the literature review, both in terms of acceptance and technical design (AUBERT et al., 2012). 
II.1 Autonomous Field Robots in Agriculture: A Qualitative Analysis of User Acceptance According to

Compatibility is expressed, for example, by the ability to combine AFR with current working practices and existing machinery or equipment. An effect of this factor on perceived usefulness as well as on perceived ease of use is assumed. Furthermore, an effect of the variables explained below is assumed to have a direct effect on behavioral intention. The farm structure is formed by two components. First, the farm size and second, the availability of resources, i.e., the resources needed to implement autonomous technology, are considered (AUBERT et al., 2012). In addition, the characteristics of the farm manager are described separately as an external factor, taking into account socio-economic variables; primarily age and educational level, as well as attitude to risk (PATHAK et al., 2019). The availability of workforce is also defined as an external factor influencing behavioral intention, since labor shortage was identified in previous studies as a factor influencing the acceptance of AFR (GRIEPENTROG, 2010; LOWENBERG-DEBOER et al., 2020). It is important to note that economic benefit as a possible factor for autonomous robotics compared to conventional systems is included in perceived usefulness but receives special attention in the survey. In particular, assumptions of a higher crop yield, cost savings, first of all in labor costs, and an increase in economic efficiency are taken into account. Based on these factors, various potential acceptance influences were assumed (Figure 1).

The data was analyzed using a qualitative content analysis with the help of the software "f4 Analysis" (GLÄSER and LAUDEL, 2010). A qualitative content analysis aims to systematically evaluate the information originating from a specific type of communication in a rule-based approach (MAYRING, 2015). In contrast to quantitative studies, statistical representativeness is not the primary objective. Rather, the idea is to analyze a problem that has, to date, hardly been studied, initially in an exploratory way, using the situations reconstructed from expert interviews to empirically investigate their experiences, opinions and attitudes. In this way, researchers create a base for possible further quantitative research (GLÄSER and LAUDEL, 2010; PATTON, 2015). The qualitative approach has been chosen in the present study, since there is hardly any data available so far for the field of investigation in the agricultural context.

The resulting data was assigned to the TAM factors described above (perceived usefulness, perceived ease of use and external factors) influencing the acceptance of AFR, with the possibility of an ex-post addition in case of newly collected information which had not been considered previously. This was important because, due to the limited literature on this particular topic, there was little evidence or connections between the factors influencing acceptance of AFR that could be determined in advance (GLÄSER and LAUDEL, 2010). 
II.1 Autonomous Field Robots in Agriculture: A Qualitative Analysis of User Acceptance According to

The evaluation of the influencing factors provided the information needed to explain the directions of potential influence in the results section below.

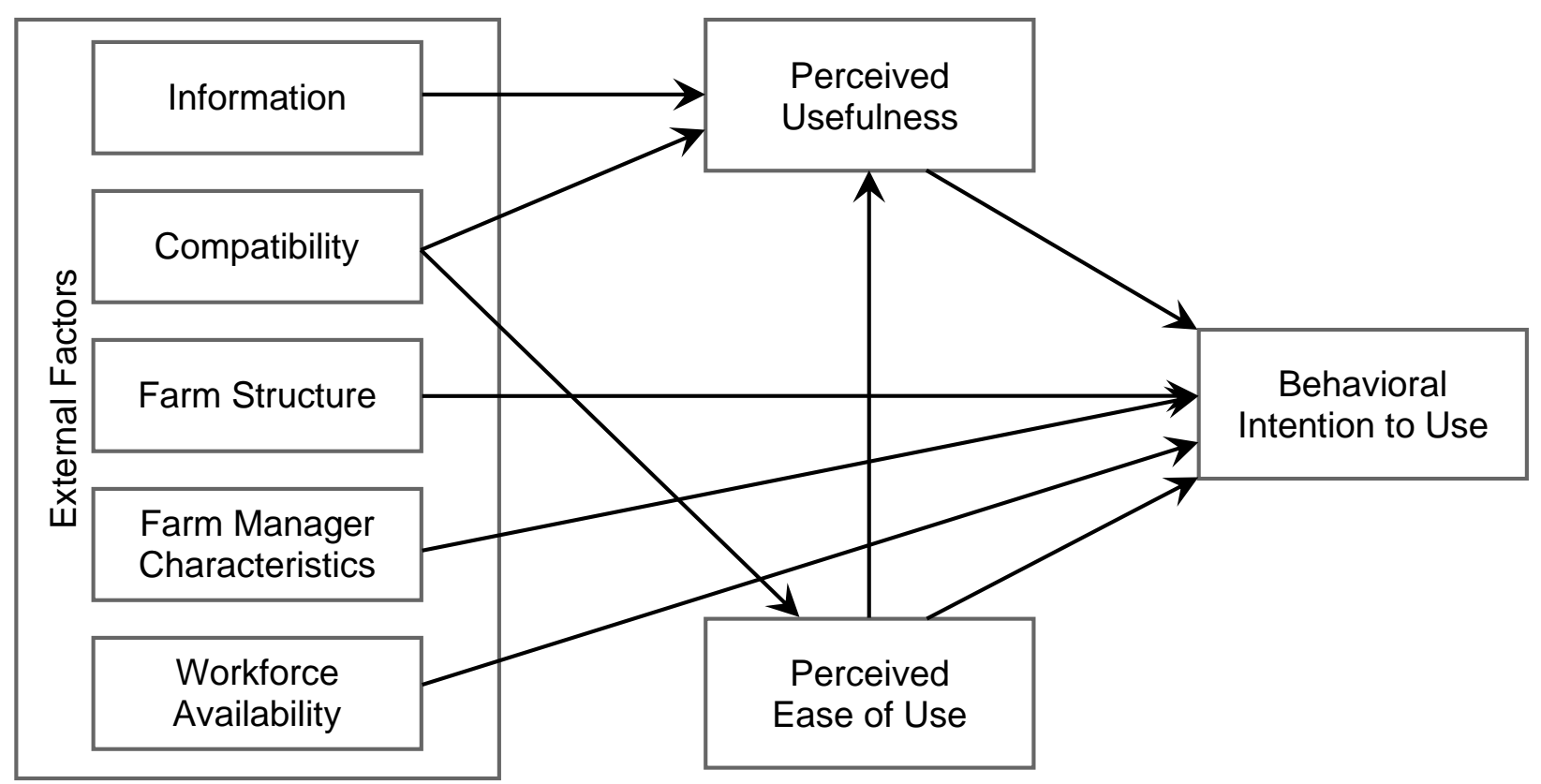

Figure 1: Modified TAM in the context of AFR

Source: Authors' illustration according to ADRIAN et al. (2005), AUBERT et al. (2012), DAVIS (1989) and RIAL-LOVERA (2018)

\section{Results}

\subsection{Sample Description}

Nine experts from agricultural machinery companies were selected for the interviews, including seven established agricultural equipment and vehicle manufacturers and two AgTech startup companies (see Table 1). The selection criteria for the established companies were direct or indirect involvement in AFR, e.g., by developing their own prototypes or investing in a corresponding AgTech startup. Also high total revenue, in order to ensure the relevance of the statements. The selection of the experts was left to the companies. 
II.1 Autonomous Field Robots in Agriculture: A Qualitative Analysis of User Acceptance According to

Table 1: Sample description

\begin{tabular}{|c|c|c|}
\hline \multicolumn{2}{|c|}{ Company } & \multirow{2}{*}{$\begin{array}{l}\text { Headquarter } \\
\text { Marktoberdorf, Bavaria (Germany) }\end{array}$} \\
\hline \multirow{6}{*}{ 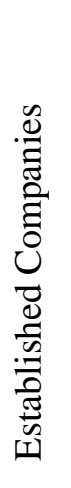 } & AGCO Fendt & \\
\hline & Amazone & Hasbergen, Lower Saxony (Germany) \\
\hline & Claas & Harsewinkel, North Rhine-Westphalia (Germany) \\
\hline & CNH Industrial & Heilbronn, Baden-Württemberg (Germany) \\
\hline & Horsch & Schwandorf, Bavaria (Germany) \\
\hline & John Deere & Kaiserslautern, Rhineland-Palatinate (Germany) \\
\hline & SDF & Lauingen, Bavaria (Germany) \\
\hline \multirow{2}{*}{ 芯 } & Deepfield Robotics & Ludwigsburg, Baden-Württemberg (Germany) \\
\hline & Naiture & Friedrichsgabekoog, Schleswig-Holstein (Germany) \\
\hline
\end{tabular}

Source: Authors' illustration

\subsection{Perceived Usefulness}

According to the experts interviewed, AFR profitability has the greatest relevance for acceptance among farmers. However, the majority of experts have doubts about AFR's economic advantages in arable farming at this stage of development: "There is simply the problem with the practical feasibility, with the economic efficiency, with the functionality of the individual applications and also the proof that it works." (E8). For example, constant logistic costs with less acreage capacity are mentioned (E1; E7). Two experts agree that crop protection with conventional machinery is much more cost-effective than using AFR and will remain so in the mid-term, unless there is a glyphosate ban (E1; E4). In contrast, possible efficiency gains from the use of AFR in certain work processes were highlighted, provided that such processes required a large amount of time (E1; E3; E6; E7). In particular, the high precision of pest detection and the possibility of 24-hour, 7-day a week operation using AFR are mentioned (E1; E3; E9). Expert 9 added that time saved could be used by farmers to further increase profitability on their farms. However, this requires the farmers to be aware of these effects (E1). The argument that AFR can save input costs such as machine costs, fertilizers, pesticides, seeds and labor cost has been largely confirmed: "Of course, at one point it is the manpower and all the machinery that I have that I can do without." (E4). But according to Expert 7, the saved salary would amount to 5-10\%. The major potential for savings is seen in the more precise application of pesticides and 
II.1 Autonomous Field Robots in Agriculture: A Qualitative Analysis of User Acceptance According to

fertilizers: "Because, in relative terms, the costs of agricultural engineering are in a ratio to the costs of the inputs, which are very much to the disadvantage of the inputs. This means that a saving of $20 \%$ in pesticides is of course an enormous amount of money." (E1). The price is perceived as a further criterion in deciding whether to use AFR: "Ultimately, it is relatively easy to break it down to the price. If the thing is too expensive and not worth it, hardly anyone will do it." (E4). The argument that AFR today are still too expensive is countered by a respondent who argues that total initial savings of 30 to $40 \%$ are already possible in certain arable work processes, such as plant protection, fertilization and seeding (E6). In addition, Experts E1, E2 and E6 point out that AFR must operate reliably and safely, so that human intervention is not required constantly: “(...) if a robot is not reliable and constantly sends out a call for help (...) then the whole thing is over quickly. " (E6).

With regard to the TAM, the assumed potential acceptance influences are reflected by the statements of many experts. The economic benefit, which is included in the factor perceived usefulness, has a direct effect on farmers' behavioral intention according to the experts (see Figure 2). Furthermore, a potential influence of perceived ease of use on perceived usefulness could be found by the expected reliability of AFR, more precisely by the fear of the need for human intervention in the autonomous process. Furthermore, effects of the categories of technology and legal framework on perceived usefulness can be determined.

\subsection{Perceived Ease of Use}

Perceived ease of use is confirmed by most experts as an important potential influencing factor (E2; E3; E4; E7; E9), although Expert 4 notes that a contractor could also take over the operation of AFR, at which point this influencing factor would be irrelevant to the farmer. Otherwise, the ease of use is to be classified as: “(...) very, very important." (E4). It is added that it must not be a solution: “(...) that can now only be operated by an engineer who has developed it." (E9). Although two respondents call for training on AFR (E2; E9), Expert 1 states that operating machinery will be easier in the future due to increasing automation. He continues that the advantages of autonomous technology would be lost if it were complicated to operate: "All I want to say [as a farmer] is, Alexa, please go to this field, execute order XL. Then, as I have already stored my farm management software. Done. That's all I want. Give me a report." (E3). In this context, one expert fears that too much dependence on technology could lead to disappointment amongst farmers, especially if they get the feeling that they are no longer: “(...) master of things.” (E7). Expert 9 adds that, given AFR's complexity, self-repair is probably no longer an option for the farmers. 
II.1 Autonomous Field Robots in Agriculture: A Qualitative Analysis of User Acceptance According to

It is demonstrated that the influence of the TAM factor ease of use on AFRs acceptance is reflected in statements from the respondents. Although different levels of importance were identified, a high level predominates. The assumed potential influence on perceived usefulness could be found, for example, by the suggestion of training programs for farmers. Possible operating methods for AFR reveals a wide range of approaches, from the transfer to a contractor up to complex operations, although the majority was in favor of a simple and intuitive operation. In addition, an influence of the sales structure on the factor ease of use became apparent through the service transfer mentioned.

\subsection{Information}

With regard to data protection and data security, several respondents made a distinction between pure machine data and farm-based agronomic data, which does not only apply to AFR: "For us, this is not a specific AFR problem, because it is already an issue today with the introduction of telemetry systems and field mapping and all the information that vehicles nowadays are collecting and possibly reporting back to the manufacturers." (E5). Many respondents agree with this statement, highlighting that farmers are already willing to share their data if there is a visible benefit for them (E2; E3; E4; E9). Potential conflicts are seen in the lack of data sovereignty in a data cloud and the danger of unauthorized access to the machines (E3; E7). Expert 6 adds that: "Data collection alone is not that bad. (...) The problem is if you can evaluate the data with AI. And depending on who then gets hold of the whole thing and pushes the interests, you can of course mess around with it quite a bit." One interviewee believes that many farmers are not yet aware of this data issue (E8).

It appears that any assumed influence of the factor information on user acceptance is very limited from the perspective of the interviewees, since agricultural machinery manufacturers already collect and use large amounts of data and no relevant relationship to user acceptance could be drawn so far. However, a danger is seen in the misuse of the information, especially in the sense of external control of autonomous agricultural machines. This applies more to large-scale autonomous technology than to small-scale autonomous technology. Nevertheless, information is important for the further development of AFR and questions of data protection and user authority should be clarified from the experts' point of view. 
II.1 Autonomous Field Robots in Agriculture: A Qualitative Analysis of User Acceptance According to

\subsection{Compatibility}

The effect of the factor compatibility is linked to technical design (E4) and the associated process dependency (E1;E3) of AFR. The range of possibilities for AFR technical design was still very wide (E4; E7; E9). Furthermore, according to Expert 2, it depends on whether only individual machines or a complete system should be autonomous. Two experts add: "Will it be more like a cable-less tractor as a dumb pulling device with appropriate equipment? Or will it be a completely different system? We think it probably only makes sense if it will be a completely different system." (E7). "But there is also simply the question: Is it really the right step to base a new technology on it, (...) our avowed three-point is the thing that everyone has to do. Or is there no other solution? Just because we've solved it this way with tractors, do we have to do it this way?" (E3). Expert 1 adds that any AFR with a specific field of application must achieve higher savings than universal AFR because of its fewer operations. The universal applicability of AFRs is regarded as an important factor for user acceptance (E8). Several experts also underline the importance of a transition period towards autonomous techniques to promote acceptance of AFR among farmers (E3; E4; E9).

Thus, the assumed potential influence of the external TAM factor compatibility on perceived usefulness could be confirmed by the required transition period for farmers. During this transition period, AFR and conventional agricultural machinery must be compatible. A potential influence of compatibility on perceived ease of use could be seen in the required technical design and process dependency of AFR.

\subsection{Farm Structure}

One expert identifies farm structure as having a direct influence on AFR acceptance, in addition to professionality on farms, since the trend towards increasing farm concentration also puts productivity pressure on farm managers (E8). Apart from farm size, field structure is also considered relevant: "The larger the fields I have and the fewer fields I have, the faster I can use robots, because the less I have to move them from field to field, the more effective it becomes. (...) This could also be the 100 hectare farm now, which simply has 100 hectares arranged around its house. This is the perfect robot farm. Not so the 500hectare farm in Bavaria that has spread out over 250 fields." (E3). Expert 1 assumes that larger farms are more likely to replace machinery and purchase robots than smaller farms because of a better resource allocation. In contrast, Expert 4 considers the size of the farm to be irrelevant if a: “(..) weeding service is offered." (E4). Larger farms would also be 
II.1 Autonomous Field Robots in Agriculture: A Qualitative Analysis of User Acceptance According to

more able to cover expected investment costs of AFR (E1; E7). Two experts explicitly do not consider farm size to be crucial, as smaller farms can also be highly modern (E2; E8).

To summarize, most experts see a positive correlation between AFR's use and increasing farm size, due to greater resource allocation. With regard to the TAM, the effect of farm structure on behavioral intention is reflected. In addition, there seems to be a potential influence on perceived usefulness in terms of investment costs and on ease of use in terms of logistic costs, whose intensity remains to be investigated.

\subsection{Farm Manager Characteristics}

The characteristics of the farm manager were assessed differently by the interviewees, although a certain degree of openness towards new developments is generally seen as encouraging (E1; E9). However, this openness varies among farmers: "Well, I am in contact with many farmers who are very open to new technologies and who promote this and also ask for it. Or even come up with suggestions themselves. And I also know farmers who say that they have been doing this for 100 years and will continue to do so." (E9). The majority of experts see a positive correlation between openness towards AFR and the level of education of farmers (E1; E2; E4; E7; E8). In addition, two of the respondents see a negative correlation between lower educational levels and increasing age (E7; E8). The majority of experts believe that younger farmers have a greater affinity for digital technologies, such as AFR, because they have grown up with them (E2; E4; E5; E6; E7; E8). While Expert 3, in contrast, assumes that farm managers aged 45 to 60 will adopt AFR the fastest, Experts 1 and 9 share a different opinion: "Well, I think that age is not really important, but rather the horizon of experience or the willingness to innovate. For us it is not always so clear that innovative farmers always have to be 25 or 35 years old, there are also innovative 5060-year olds." (E1).

Although these results indicate the relevance of the effect of the farm manager's characteristics as an expression of age and educational level, this expression is not entirely clear. Regarding age, a higher acceptance is increasingly assumed among younger farmers. With regard to the level of education, a slightly lower relevance was found among the respondents. In summary, it is noticeable that the experts do not usually attribute the influence of the farm manager's characteristics directly to the behavioral intention assumed but rather an effect in the form of the farm manager's openness towards new technology on perceived usefulness and perceived ease of use (see Figure 2). 
II.1 Autonomous Field Robots in Agriculture: A Qualitative Analysis of User Acceptance According to

\subsection{Workforce Availability}

Due to experts' frequent differentiation between arable farming and special crops, the factor workforce was examined in a differentiated manner as well. The availability of workforce in agriculture is perceived as a general problem: "But it will still be difficult to find workers at all, (...) so that the considerations can go as far as to say that a robot can also do such work, for example." (E8). Expert 7 sees the monotony of work, especially on largescale farms (> 1,000 hectares), as an obstacle for young people working in agriculture. Two experts assume that salary costs will rise in the future (E8; E9), whereas Expert 1 argues that in the case of Germany, first, machine operators will continue to be affordable and, second, as mentioned above, the skill level will decrease due to the higher degree of automation in agricultural machinery (E1). It would rather result in: “(...) different or additional skills being required from employees." (E5). Extending the analysis to special crop cultivation reveals a more uniform picture: "I think that at the moment it can save mainly handwork. And that costs. And it's only used in organic or special crops. At least for now." (E4). It is noted that the workforce: "(...) are coming from further and further away and the effort is increasing." (E1). Expert 1 continues, if, as a result of political constraints, workers are not allowed in any region where AFR operates, this may also have a positive effect on the adoption of AFR.

The experts see only a limited influence of the factor workforce on the acceptance of AFR in relation to arable farming in Germany. However, in the field of special crops this relationship is considered to be of high importance, since the effort increases with the number of workers required. This indicates a potential influence of perceived ease of use on behavioral intention, as assumed. Furthermore, there might be a potential influence on behavioral intention due to the fact that AFR are perceived as a response to workforce shortage in agriculture. 
II.1 Autonomous Field Robots in Agriculture: A Qualitative Analysis of User Acceptance According to

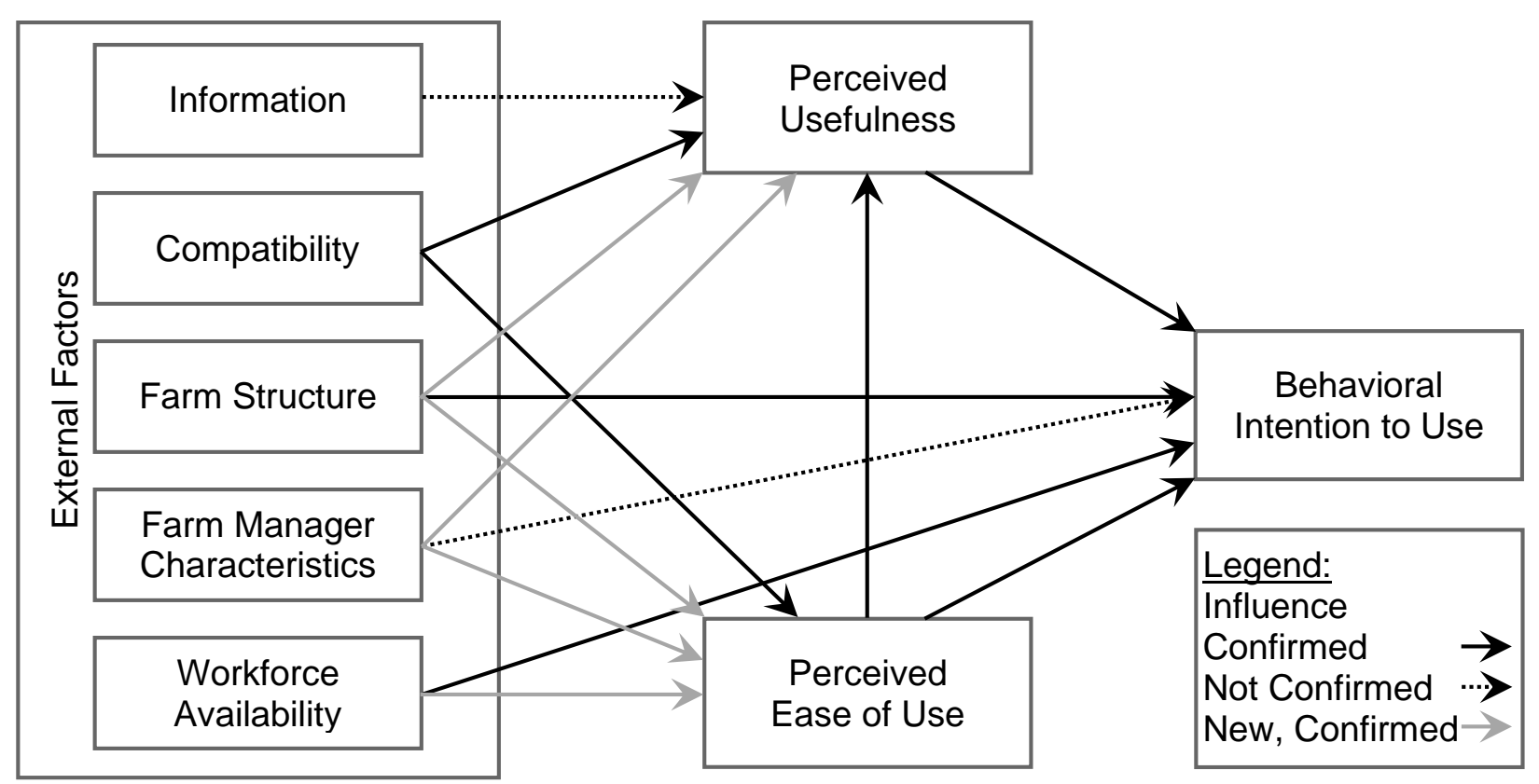

Figure 2: Examined TAM in the context of autonomous field robotics

Source: Authors' illustration according to ADRIAN et al. (2005), AUBERT et al. (2012), DAVIS (1989) and RIAL-LOVERA (2018)

\section{Discussion}

The opportunities for data collection and evaluation through SFT such as AFR are gradually increasing competition in the agricultural machinery market from not only new AgTech startups but also from established IT giants such as IBM or Google. In order to remain dominant in the market, existing agricultural engineering companies should be careful not to fall victim to such disrupters. Therefore, the aim of this exploratory study was to provide a qualitative understanding of the factors influencing the ex-ante user acceptance of AFR from the perspective of the agricultural machinery companies. For this purpose, guidelinebased interviews were conducted with nine experts from the German agricultural machinery sector. The interviews were based on the TAM according to DAVIS (1989), supported by external factors derived from the available scientific literature on this topic. In summary, it was found that the topic's wide scope means that a large number of factors influence farmers' acceptance from the point of view of agricultural machinery manufacturers, with perceived usefulness being regarded as the greatest motivation for using AFR. Even if future need for AFR is assumed by all experts, the economic advantages of autonomous agricultural technology over conventional technology are not plausible in the medium term from the experts' point of view. The interviewees do not expect AFR to be relevant in 
II.1 Autonomous Field Robots in Agriculture: A Qualitative Analysis of User Acceptance According to

arable farming for at least 10 years, although earlier in special crops. AFR's future market share will depend above all on legislation regarding autonomous technologies. If AFR are used on a large scale in arable farming, this will very likely lead to changes in the existing business models affecting the end customer. Most of the assumed potential interactions of the factors surveyed in the TAM on user acceptance of AFR have been confirmed by the experts (Figure 2). This again demonstrated the transferability of the TAM to agricultural research as a model for explaining technology acceptance (e.g., ALAMBAIGI and AHANGARI, 2015). However, there were also differences and newly revealed potential influences.

Perceived usefulness was identified as the most mentioned potential influencing factor, which is expressed primarily as economic benefit in terms of investment costs and input savings of and through AFR. This confirms the results of PIERPAOLI et al. (2013), who used a TAM to investigate the factors influencing the ex-ante acceptance of PAT. The price of AFR assumed by the experts differed widely. One reason for this could be that AFR is still at an early stage of development for certain workflows (RÜBCKE VON VELTHEIM et al., 2019). Nevertheless, a strong dependence on future user acceptance on price was revealed, which is in line with the results of RIAL-LOVERA (2018), who identified investment costs as a barrier to the adoption of AFR. Despite the cost and efficiency advantages of AFR assumed by the experts and various studies (GAUS et al., 2107; LOWENBERG-DEBOER et al., 2020; SHOCKLEY et al., 2019), an advantage of autonomous agricultural technology over existing conventional agricultural technology in arable farming is not perceived at present, nor in the mid-term. Therefore, and in addition to DöRR et al. (2019) who argue that farmers would not perceive the user benefits of autonomous agricultural technology, it can be concluded that better communication of economic advantages could promote greater acceptance among farmers. The experts also assigned ease of use as highly relevant for user acceptance, which is in line with other agricultural studies using the TAM (e.g., ADRIAN et al., 2005; AUBERT et al., 2012). At the same time, the majority of the experts forecast a decreasing level of operator qualification, which also depends on the business model (e.g., outsourcing of certain processes to service providers). However, this confirms the findings of DEVITT (2018) and DÖRR et al. (2019), according to whom the relationship between operator and machine will be weakened by the introduction of AFR and a change from machine operator to a less skilled system operator will take place. Nevertheless, the experts demand to retain the possibility of human intervention in the work process. There is greater consensus among the experts on the compatibility factor, since they assume that, particularly in the predicted transition period from semi-autonomous systems to fully autonomous ones, compatibility with conventional technology will be essential for AFR 
II.1 Autonomous Field Robots in Agriculture: A Qualitative Analysis of User Acceptance According to

acceptance among farmers. This is confirmed by RIAL-LOVERA (2018), who cites a lack of compatibility and AFR standardization as a possible barrier to acceptance among farmers. The respondents underlined the dependence of compatibility on the intended area of application, with a distinction being made between special AFR with less required compatibility and universal AFR with more required compatibility. DÖRR et al. (2019) additionally anticipate a future increase in universal AFR approaches. The farm structure showed a diverse picture, with the larger share of experts associating higher acceptance with increasing farm size. This is in line with previous studies on PAT (CAFFARO and CAVAllo, 2019). Larger farms are considered to have better resource allocation, which allows them to invest more in new technologies. DöRR et al. (2019) also take differences in farm structures as an acceptance factor, which will lead to different introductions of AFR regionally. In contrast, SHOCKLEY et al. (2019) provide evidence for higher profitability from operating AFR on smaller farms, as smaller farms are still able to achieve economies of size with autonomous machinery. Regarding farm manager's characteristics, age and level of education were considered to be of some relevance to AFR's acceptance process, even though there was disagreement among experts. DEVITT (2018) findings showing that younger, better educated and more technologically advanced farmers are more likely to use new technologies can only be partially confirmed. The effects of the farmers' level of education, as recorded in PAT studies, could not be clearly confirmed (CAFFARO and CAVALlO, 2019). However, a certain open-mindedness towards new technologies amongst farmers was mentioned by the experts as promoting acceptance and traditional attitudes as inhibiting acceptance. DEVITT (2018) unanimously confirmed the lack of ability to generate trust in AFR as an important cognitive barrier to acceptance. Demonstrations of the new technology could be used to overcome possible acceptance barriers due to the farm manager's personal characteristics. Among the external factors, availability of workforce was identified as an important driver in the user acceptance process, although the respondents believe that this factor will be more important in special crops than in arable farming. Regional differences are confirmed by GRIEPENTROG (2010), who found that regions with high labor availability and low wages will be less attractive for the introduction of AFR. The influence of workforce availability on ease of use was found to be caused by the high coordination effort of a large number of seasonal workers in special crop cultivation. Confirming this, RIAL-LOVERA (2018) identified workforce availability and rising wages in agriculture as key drivers for the adoption of AFR. Only for the factor information no recognizable influence could be found. This may be due to the fact that agricultural machinery manufacturers have found from experience that a lot of production-related and general data 
II.1 Autonomous Field Robots in Agriculture: A Qualitative Analysis of User Acceptance According to

is already collected from farmers as potential AFR users on a daily basis, either by the manufacturers themselves or through the various applications on farmers' smartphones.

However, despite the interesting results regarding the ex-ante user acceptance of AFR, as with most data collected empirically, a number of limitations must be taken into account when interpreting the findings. For instance, the majority of the agricultural machinery manufacturers interviewed are established companies which, unlike the two AgTech startups, may have a conservative perspective on this new technology and may have little experience with farmers using AFR. It can also be questioned to what extent agricultural machinery manufacturers are suitable as experts to predict the behavioral intention of farmers. However, since the experience of technical innovations has been shown to influence its acceptance, the results could differ if the sample composition were to change (e.g., more AgTech startups and less established agricultural machinery companies) (MELENHORST and BouWHUIS, 2004). The concept of the study also offers a target for misinterpretation. Thus, despite all efforts to assign the experts' statements as objectively as possible to the factors formed, a certain subjectivity can never be excluded. Nevertheless, as mentioned above, the present qualitative study is only an exploratory study designed to identify and assess the ex-ante acceptance of AFR and its influencing factors. No claim is made to representativeness.

Nevertheless, the results can be used to help AFR manufacturers and political decisionmakers to promote the diffusion of AFR. It also offers starting points for further research in the area of acceptance of autonomous machinery in agriculture. Thus, it could not be clearly identified whether a high level of ease of use could outweigh a lack of perceived usefulness or whether there would be an acceptance of this technology given less ease of use. From a methodological point of view, it would be valuable to carry out further quantitative research. 
II.1 Autonomous Field Robots in Agriculture: A Qualitative Analysis of User Acceptance According to

\section{References}

AdRIAN, A. M., NORWOOD, S. H. and P. L. MASK (2005): Producers' perceptions and attitudes toward precision agriculture technologies. In: Computers and Electronics in Agriculture 48 (3): 256-271, doi: 10.1016/j.compag.2005.04.004.

Alambaigi, A. and I. Ahangari (2015): Technology Acceptance Model (TAM) As a Predictor Model for Explaining Agricultural Experts Behavior in Acceptance of ICT. In: International Journal of Agricultural Management and Development 6 (2): 235-247, doi: 10.22004/ag.econ.262557.

Aubert, B. A., SChroeder, A. and J. Grimaudo (2012): IT as enabler of sustainable farming: An empirical analysis of farmers' adoption decision of precision agriculture technology. In: Decision Support Systems 54 (1): 510-520, doi: 10.1016/j.dss.2012.07.002.

Benbasat, I. and A. S. DeXTER (1986): An Investigation of the Effectiveness of Color and Graphical Information Presentation under Varying Time Constraints. In: MIS Quarterly 10 (1): 59-83, doi: $10.2307 / 248881$.

BukChin, S. and D. Kerret (2018): Food for Hope: The Role of Personal Resources in Farmers' Adoption of Green Technology. In: Sustainability 10 (5): 1615, doi: 10.3390/su10051615.

BuRWOOD-TAYlor, L., LeCLERC, R. and M. TILNEY (2016): AgTech investing report: Year in review 2015. AgFunder. URL: https://research.agfunder.com/2015/AgFunder-AgTech-Investing-Report-2015.pdf (accessed 15 February 2020).

CAfFaro, F. and E. CAVALLO (2019): The Effects of Individual Variables, Farming System Characteristics and Perceived Barriers on Actual Use of Smart Farming Technologies: Evidence from the Piedmont Region, Northwestern Italy. In: Agriculture (MDPI) 9 (5): 111, doi: 10.3390/agriculture9050111.

ChutTur, M. (2009): Overview of the Technology Acceptance Model. Origins, Developments and Future Directions. In: Sprouts: Working Papers on Information Systems 9 (37): 1-21.

DAVIS, F. D. (1989): Perceived Usefulness, Perceived Ease of Use, and User Acceptance of Information Technology. In: MIS Quarterly 13 (3): 319-340, doi: 10.2307/249008.

DeVITT, S. K. (2018): Cognitive Factors that Affect the Adoption of Autonomous Agriculture. In: Farm Policy Journal 15 (2): 49-60.

Dörr, J., Fairclough, B., Henningsen, J., Jahić, J., Kersting, S., Menning, P., PePer, C. and F. ScholTEN-BusCHHOFF (2019): Scouting the Autonomous Agricultural Machinery Market. Fraunhofer IESE and Kleffmann Group, Kaiserlautern/Lüdinghausen.

ErL, T., Puttini, R. and Z. Mahmood (2019): Cloud Computing: Concepts, Technology \& Architecture. Prentice Hall, New Jersey.

FuCHS, A. (2018): Autonome Landtechnik. Editorial. In: ATZheavy duty 11 (2), doi: 10.1007/s35746-018$0023-1$.

Gaus, C.-C., Minßen, T.-F., URso, L.-M., DE WiTTE, T. and J. Wegener (2017): Mit autonomen Landmaschinen zu neuen Pflanzenbausystemen. Johann Heinrich von Thünen-Institut, Institut für mobile Maschinen TU Braunschweig und Julius Kühn-Institut, Braunschweig.

GLÄSER, J. and G. LAUDEL (2010): Experteninterviews und qualitative Inhaltsanalyse als Instrumente. Lehrbuch, fourth edition. VS Verlag für Sozialwissenschaften, Wiesbaden.

Griepentrog, H.W. (2010): Automatisierung in der Außenwirtschaft. In: MOLNAR, C. and M. PIKART-MÜLLER (Eds.): Automatisierung und Roboter in der Landwirtschaft. Kuratorium für Technik und Bauwesen in der Landwirtschaft, Darmstadt: 25-33.

Kester, C., Griepentrog, H. W., Hörner, R. and Z. TunCER (2013): A survey of future farm automation - a descriptive analysis of survey responses. In: J. V. STAFFORD (Ed.): Precision Agriculture' 13. Wageningen Academic Publishers, Wageningen: 785-792, doi:10.3920/978-90-8686-778-3_97. 
II.1 Autonomous Field Robots in Agriculture: A Qualitative Analysis of User Acceptance According to

KING, A. (2017): Technology: The Future of Agriculture. In: Nature 544 (7651): 21-23, doi: 10.1038/544S21a.

KuCZERA, C. (2006): The influence of the social environment on farm decisions of farmers. Margraf Publishers, Weikersheim: 56-171.

Lowenberg-DeBoer, J., HuAng, I. Y., Grigoriadis, V. and S. Blackmore (2020): Economics of robots and automation in field crop production. In: Precision Agriculture 21 (2020), doi: 10.1007/s11119-01909667-5.

MAYRING, P. (2015): Qualitative Inhaltsanalyse. Grundlagen und Techniken. Beltz, Weinheim.

Melenhorst, A.-S. and D. G. Bouwhuis (2004): When do older adults consider the internet? An exploratory study of benefit perception. In: Gerontechnology 3 (2):89-101, doi: 10.4017/gt.2004.03.02.004.00.

Miller, L. H. (1977): A Study in Man-Machine Interaction. In: AsSOCIATION FOR COMPUTING MACHINERY (Ed.): Proceedings of the National Computer Conference, Dallas, Texas, USA, 13-16 June 1977: 409421, doi: 10.1145/1499402.1499475.

PAthak, H. S., BRown, P. and T. BeST (2019): A systematic literature review of the factors affecting the precision agriculture adoption process. In: Precision Agriculture 13 (9): 1-25, doi: 10.1007/s11119-01909653-x.

PATTON, M. Q. (2015): Qualitative research \& evaluation methods: integrating theory and practise. SAGE, Los Angeles.

PAustian, M. and L. THEUVSEN (2016): Adoption of precision agriculture technologies by German crop farmers. In: Precision Agriculture 18 (5): 701-716, doi: 10.1007/s11119-016-9482-5.

PHAM X. and M. STACK (2017): How data analytics is transforming agriculture. In: Business Horizons 61 (1): 125-133, doi: 10.1016/j.bushor.2017.09.011.

Pierpaoli, E., Carli, G., Pignatti, E. and M. Canavari (2013): Drivers of Precision Agriculture Technologies Adoption: A Literature Review. In: Procedia Technology 8: 61-69, doi: 10.1016/j.protcy.2013.11.010.

Redhead, F., Snow, S., Vyas, D., Bawden, O., Russell, R., Perez, T. and M. Brereton (2015): Bringing the Farmer Perspective to Agricultural Robots. In: JINWOO, K. I. and W. WOO (Eds.): Proceedings of the 33rd Annual ACM Conference Extended Abstracts on Human Factors in Computing Systems. ACM Press, New York: 1067-1072, doi: 10.1145/2702613.2732894.

RIAL-LoverA, K. (2018): Agricultural Robots: Drivers, Barriers and Opportunities for Adoption. In: N. TREMBLAY (Ed.): Proceedings of the 14th International Conference on Precision Agriculture, Montreal, 2427 June 2018. The International Society of Precision Agriculture, Monticello Illinois.

RÜBCKe von Veltheim, F., Theuvsen, L. and H. Heise (2019): Akzeptanz autonomer Feldroboter im Ackerbaueinsatz: Status quo und Forschungsbedarf. In: Berichte über Landwirtschaft 97 (3): 1-18, doi: 10.12767/buel.v97i3.248.

Shockley, J. M., Dillon, C. R. and S. A. Shearer (2019): An economic feasibility assessment of autonomous field machinery in grain crop production. In: Precision Agriculture 20 (2019): 1068-1085, doi: 10.1007/s11119-019-09638-w. 


\section{II.2 The AgTech Startup Perspective to Farmers Ex-Ante Acceptance Process of Autonomous Field Robots}

Friedrich Rübcke von Veltheim und Heinke Heise

Geleisteter Eigenanteil an dem Beitrag

\begin{tabular}{|c|c|c|c|c|c|}
\hline $\begin{array}{l}\text { Literatur- } \\
\text { recherche }\end{array}$ & $\begin{array}{l}\text { Entwicklung } \\
\text { des } \\
\text { Forschungs- } \\
\text { rahmens }\end{array}$ & $\begin{array}{l}\text { Konzept und } \\
\text { Design der Stu- } \\
\text { die }\end{array}$ & $\begin{array}{l}\text { Auswahl, Aus- } \\
\text { führung und } \\
\text { Entwicklung } \\
\text { von Methoden }\end{array}$ & $\begin{array}{l}\text { Interpretation } \\
\text { der } \\
\text { Ergebnisse }\end{array}$ & $\begin{array}{l}\text { Konzept und } \\
\text { Schreiben des } \\
\text { Artikels }\end{array}$ \\
\hline$>90 \%$ & $>75 \%$ & $>75 \%$ & $90 \%$ & $>75 \%$ & $>75 \%$ \\
\hline
\end{tabular}

Dieser Beitrag ist so oder in ähnlicher Fassung veröffentlicht in der wissenschaftlichen Zeitschrift ,Sustainability“ 2020 (12). 


\section{Inhalt}

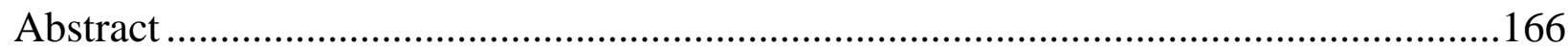

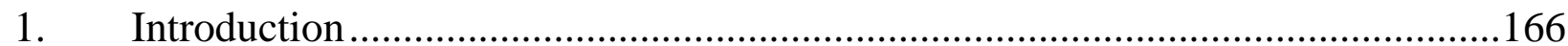

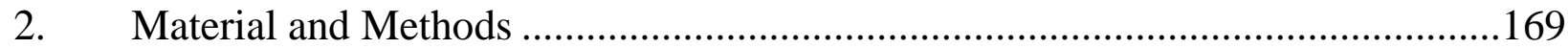

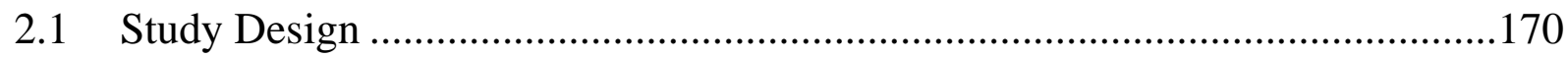

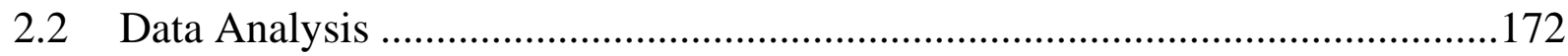

2.3 Sample Description ……………………………........................................173

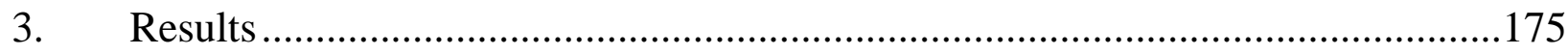

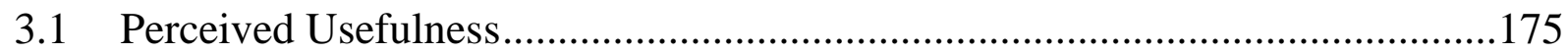

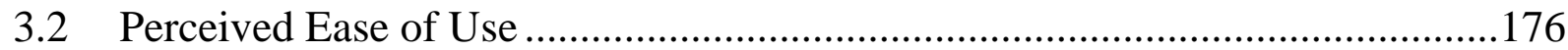

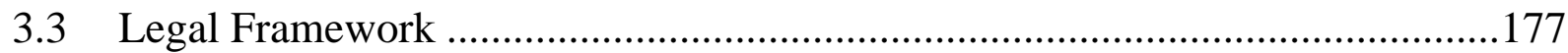

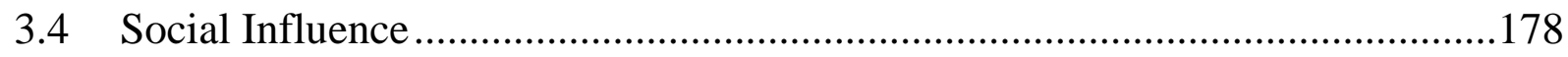

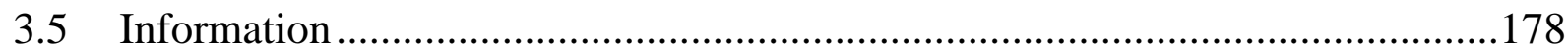

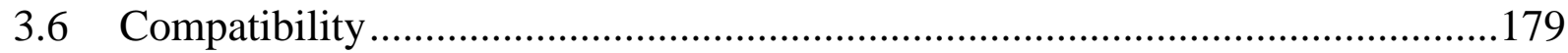

$3.7 \quad$ Farm Manager Characteristics .....................................................................180

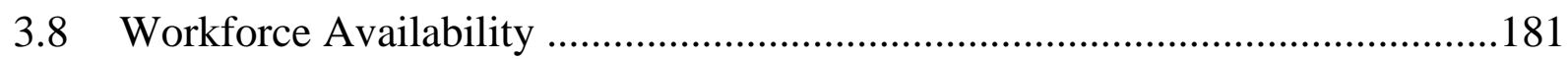

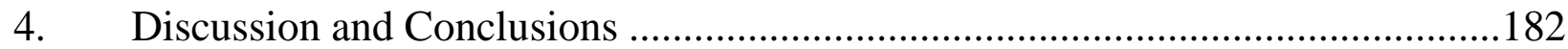

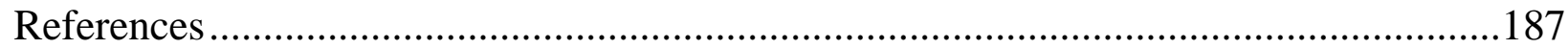

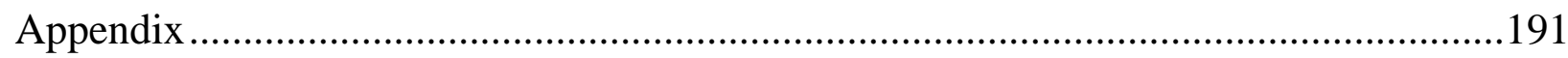




\begin{abstract}
Autonomous vehicles not only provide a new impetus in the development of car models in the automotive industry - even in agriculture there has recently been talk of AFR. Great expectations are placed on these digital assistants from a wide variety of perspectives. However, it is still unclear whether they will make the transition from market niches to broad-based distribution. Apart from various factors, this depends on user acceptance of this new technology expected by the innovators, since this is likely to be essential for the further development of AFR. For this purpose, the ex-ante user acceptance of farmers from the perspective of various AgTech startups with AFR involvement in Europe was investigated in this exploratory and qualitative study. The TAM served as the basis for the developed interview guideline. In summary, the results confirm that a variety of factors potentially influence farmer acceptance and AFR diffusion from the perspective of AgTech startups, with perceived usefulness being considered the main motivation for using AFR. The interviewed experts believe that AFR will initially be used in crops that have relatively high costs for crop protection treatments before becoming economically attractive for other crops. The basic prerequisite for a successful market launch is an adjustment of the legal framework, which sets standards in relation to AFR and thus, provides security in the production process. The results could support political decision-makers in dealing with this new technology and AFR manufacturers in the promotion of AFR.
\end{abstract}

\title{
Keywords
}

Acceptance; AgTech startups; Autonomous; Robot; TAM

\section{Introduction}

The megatrend of digitalization has not stopped at the agricultural sector. Rapidly developing technologies and infrastructures are opening up new perspectives that could even lead to a rethinking of entire farming systems (DUCKETT et al., 2018). For example, tractors as the main work equipment for arable farmers are slowly but surely being joined in their development by highly accurate, sensor-based, and intelligently linked field robots that can perform certain tasks autonomously, much more precisely, and much more sustainably either alone or in swarms with other units. The development of such AFR, which are becoming increasingly economically attractive, is mainly being driven by the growing demand for qualified workers in agriculture and the public debate about the impact of farming practices on the environment (e.g., the use of pesticides, over-fertilization, and soil compaction) 
and thus, addresses all three pillars of sustainability (economic, environmental, and social) (LOWENBERG-DeBoer et al., 2020). Social majorities can generate political pressure, which can lead to legal changes in pesticide and fertilizer application and force farmers to adapt their farming practices. AFR, some of which are still at the prototype stage, can perform specific tasks without an operator, apply pesticides on a plant-by-plant basis, or control weeds mechanically, which is why AFR could become more important in the future (REDHEAD et al., 2015). With its autonomous operation, these machines reduce the workload of farmers and protect them from unnecessary contact with harmful chemicals. Due to AFR's better scalability, smallholder farms, which have often been considered uneconomical, could become more economically attractive by adopting such robots, which could lead to a rethink in process in the agricultural sector, away from the motto: "bigger is better" (KING, 2017).

However, the introduction of autonomous technologies in agriculture is also accompanied by some concerns. For instance, although the driving of farm machinery would be abolished, new tasks would be added, such as AFR's monitoring and programming, for which farmers may lack qualified staff (REDHEAD et al., 2015). In addition, DEVITT (2018) fears that farmers may not be able to trust unmanned robots working out of sight and that by handing over tasks to artificial intelligence (AI), they would lose agricultural knowhow in the long run and thus, suffer an even greater loss of social recognition. A similar conclusion was reached by KESTER et al. (2013), who, using the example of special crops, revealed a conflict between the farmers' support of efficiently operating AFR and a lack of confidence in such technologies.

Despite the concerns expressed above, there is broad agreement that AFR will claim its place in modern agriculture in the future, but it remains unclear when and to what extent this will happen. For instance, the KING (2017) argues that the initial reluctance of established agricultural machinery manufacturers to develop AFR is due to the fact that their existing business models are being compromised. In addition, the risk of damaging reputation by a possible malfunction of the first AFR is much higher for established agricultural machinery manufacturers than for largely unknown AgTech ${ }^{1}$ startups. However, AFR also offer a whole range of new business models for agricultural machinery manufacturers, such as on-demand supply of AFR to farmers in return for a rental fee or as a cloud-based payper-use model (ERL et al., 2013).

\footnotetext{
${ }^{1}$ AgTech (Agricultural Technology) is used in this article as a generic term for all technical innovations affecting the data-driven, networked, digital agriculture of the future.
} 
Another opportunity can be seen in the ongoing development of agricultural engineering through a disaggregated approach in the form of several small robot units where large machines hit the legal size and weight limits (MINBEN et al., 2015). Since digital technologies such as AFR collect and analyze large amounts of data in order to fully unfold their potential, not only AgTech startups but also large IT companies such as IBM and Google are competing with established agricultural machinery manufacturers. As these new players will claim their share of the market, it is all the more important for the current market leaders to assess whether there is indeed a future market for AFR and, if so, to what extent (BURWOOD-TAYLOR et al., 2015). Therefore, when estimating the factors influencing the ex-ante acceptance of AFR by farmers, the perspective of various AgTech startups as new market entrants appears to be highly interesting.

However, there have only been a few studies on the technology acceptance process in relation to AFR in agriculture so far. REDHEAD et al. (2015) attribute this to the fact that this is a new, potentially disruptive technology and that not much is known about how AFR could be integrated into current farming practices. Therefore, they conducted contextual interviews with nine farmers from large-scale farms in Queensland (Australia). The farmers interviewed were particularly interested in the reduction of weed control costs and the time saved by the absence of an operator when using AFR. However, they also feared the reliability of the small robot units in an uncontrolled environment, the time required for monitoring, the complexity of AFR as well as the lack of required infrastructure in terms of high-speed mobile data networks. Overall, however, they were enthusiastic about a possible adoption of AFR, which was particularly true for farmers who were already using PAT on their farms. This can be confirmed by the findings of SALIMI et al. (2020), according to which farmers are more likely to adopt automated technologies the more they understand their usefulness. Therefore, the authors surveyed 378 people with agricultural backgrounds in Iran in 2020 about factors influencing the adoption of agricultural automation. In 2018, RIAL-LOVERA surveyed 14 agricultural stakeholders in California (USA) on their AFR acceptance behavior and revealed labor shortages and rising labor costs, as well as a lack of awareness of the potential benefits of AFR and the lack of compatibility between agricultural equipment as the main reasons for an ex-ante acceptance of AFR by farmers. Using a wider definition of the term, CAFFARO and CAVALLO (2019) conducted a survey on SFT at a northern Italian agricultural fair and found that the sociodemographic characteristics of farmers influence their adoption behavior of SFT. Overall, it can be concluded that there is a research deficit in the investigation of the technology acceptance process and diffusion of AFR in agriculture, as most studies focus only on the effect of 
certain facets and tend to ignore the multidimensionality of the acceptance process, leading to imperfect understanding of the underlying complexity of acceptance factors and diffusion barriers (BUKCHIN and KERRET, 2018; PATHAK and BROWN, 2019).

One group whose perspective appears particularly interesting in this context is, in addition to farmers (as buyers), the AgTech startups themselves (as suppliers). Following RoGERS' (1995) theory of the diffusion of innovations, the identification and successful targeting of the right group of farmers should be a top priority for suppliers in order to invest their resources into attracting the right people. In addition, the supplier's knowledge of existing drivers and barriers to the diffusion of AFR as an essentially affected party is usually higher than among other stakeholders. AFR are currently in an early market introduction phase, in which it will be decided whether the "critical mass" (ROGERS, 1995) of adoptions will be reached and the technology will be successfully established to a broader market or not.

The aim of this paper is therefore to examine first the barriers to the diffusion of AFR by providing insights into the ex-ante acceptance process of AFR. To this end, the present study examines various acceptance factors influencing the technology's diffusion from the perspective of AFR-developing AgTech startups. Since it is especially the user acceptance expected and already experienced by the suppliers that is essential for the further development and implementation of AFR, AgTech startups have collected data from farmers themselves. By interviewing the startups, it is possible to obtain aggregated knowledge about the acceptance of many farmers. Therefore, the data have been gathered through qualitative expert interviews at the International Forum of Agricultural Robotics (FIRA) in Toulouse (France) in December 2019.

\section{Material and Methods}

Due the relatively small number of AgTech startups with AFR involvement in Europe and a lack of empirical research on the acceptance of AFR, a qualitative approach for data collection and analysis was chosen: expert interviews using a semi-standardized guideline to specify the wording and order of the questions, thus ensuring a uniform procedure (MAYRING, 2015). Besides the fact that a quantitative survey was not suitable given the relatively small statistical population, expert interviews offer several advantages, since answering open questions requires higher cognitive effort from the respondents than answering closed questions and can therefore reveal more in-depth information (BARTH, 1998). In order to collect the data, we conducted interviews with experts from ten different AgTech startups specialized in AFR at the International Forum for Agricultural Robotics 
(FIRA) in Toulouse (France) in December 2019. The open and neutral formulated questions were split into six thematic blocks: drivers and barriers, economics, environmental impacts, legal constraints, socioeconomic impacts, and technology. Following a brief introduction to the topic, the experts were interviewed in person, leaving them free to add questions or topics not covered in the interview guide. The results were extracted using a qualitative content analysis according to (GLÄSER und LAUDEL, 2010; MAYRING, 2015), which is described in more detail in Section 2.2. For this purpose, individual statements from the interviews are assigned to previously developed, theory-based categories (described as factors in Section 2.1) after summarizing and aggregating the information.

\subsection{Study Design}

This 14 question-long guide (see Appendix) was based on the factors influencing the ex-ante user acceptance of new technologies based on the TAM (DAVIS, 1989). These factors cover perceived usefulness, perceived ease of use, and external factors. Since the subject of this study is the future use of AFR, it can be assumed that farmers have had little or no experience in dealing with this new technology, which is why the factors of attitude toward using and actual system use have been excluded from this analysis. In addition, ChutTur (2009) was able to demonstrate a direct influence of perceived usefulness and perceived ease of use on the behavioral intention. According to DAVIS (1989), the perceived usefulness is the subjective probability with which a potential user will adopt a certain technology (in this case AFR) to improve his work performance in an organizational context. In contrast, the perceived ease of use describes the level of simplicity of a technology expected by a potential user. Both perceived usefulness and ease of use can be influenced by various external factors (BENBASAT and DEXTER, 1986; MiLler, 1977; SALIMI et al., 2020; VERMA and SINHA, 2018). In order to address the diversity of perceived usefulness, this study explicitly addresses the underlying facets of an economic benefit in terms of, for example, higher harvest expectations, (labor-) cost savings or an increase in economic efficiency, and the facets of an environmental benefit. The effect of perceived ease of use on perceived usefulness is assumed to be positive, since a higher degree of simplicity of a technology is beneficial to its usefulness (ADRIAN et al., 2005; RIAL-LOVERA, 2018; SALIMI et al., 2020; VERMA and SINHA, 2018). The following external factors have been added based on the available literature on the topic: compatibility, farm manager characteristics, information, legal framework, social influence, and workforce availability (Aubert et al., 2012; CAFFARO and CAVAllo, 2019; Devitt, 2018; Kester et al., 2013; PATHAK et al., 2019; PAUSTIAN and THEUVSEn, 2016; REDHEAD et al., 2015; RiAL-LovERA, 2018; SALIMI et al., 2020; VERMA and SINHA, 2018). The external factor compatibility 
takes findings from diffusion theory into account, which has shown that the technical design strongly influences user acceptance (AUBERT et al., 2012). Compatibility means the possibility of combining AFR with existing technology and thus, successfully integrating it into working practice. Therefore, we expect an effect on the perceived usefulness and the perceived ease of use. As another external factor, we have introduced the farm manager's characteristics in order to meet socioeconomic aspects such as age, educational level, and risk aversion (PATHAK et al., 2019). Following an approach from PAT research, the external factor information represents the data collected by AFR and its strategic operational use as well as associated data protection and data autonomy, for which we assume a direct influence on the perceived usefulness. The sociopolitical discussion on chemical plant protection products in Europe is leading to increasing legal restrictions on the use of pesticides in agriculture (e.g., Germany: BMEL, 2020). In addition, the use of autonomous technologies on public roads is still not regulated in a way that is appropriate to the present time, which is why we assume that the legal framework has an influence on perceived usefulness (ALT, 2018). We therefore defined the external factor legal framework as the degree to which a farmer believes that organizational and legal infrastructures exist to support the use of AFR. Social influence was taken into account as a further external factor to counteract the limitation of the TAM to ignore the social environment. The fact that the social environment has an influence on the acceptance of technology has been proven several times with the follow-up models TAM2 and the UTAUT. Furthermore, farmers are influenced in their strategic decisions by their social environment (KUCZERA, 2006), which is why we assume a direct relationship between social influence and perceived usefulness. The availability of workforce has been repeatedly cited in the screened literature as a driver for the development and adoption of AFR, which is why a direct influence on the behavioral intention to use AFR is assumed from this fifth and last external factor in this study (GRIEPENTROG, 2010; LOWENBERG-DEBOER et al., 2020). The assumptions based on the factors mentioned above are shown in the following figure (Figure 1). 


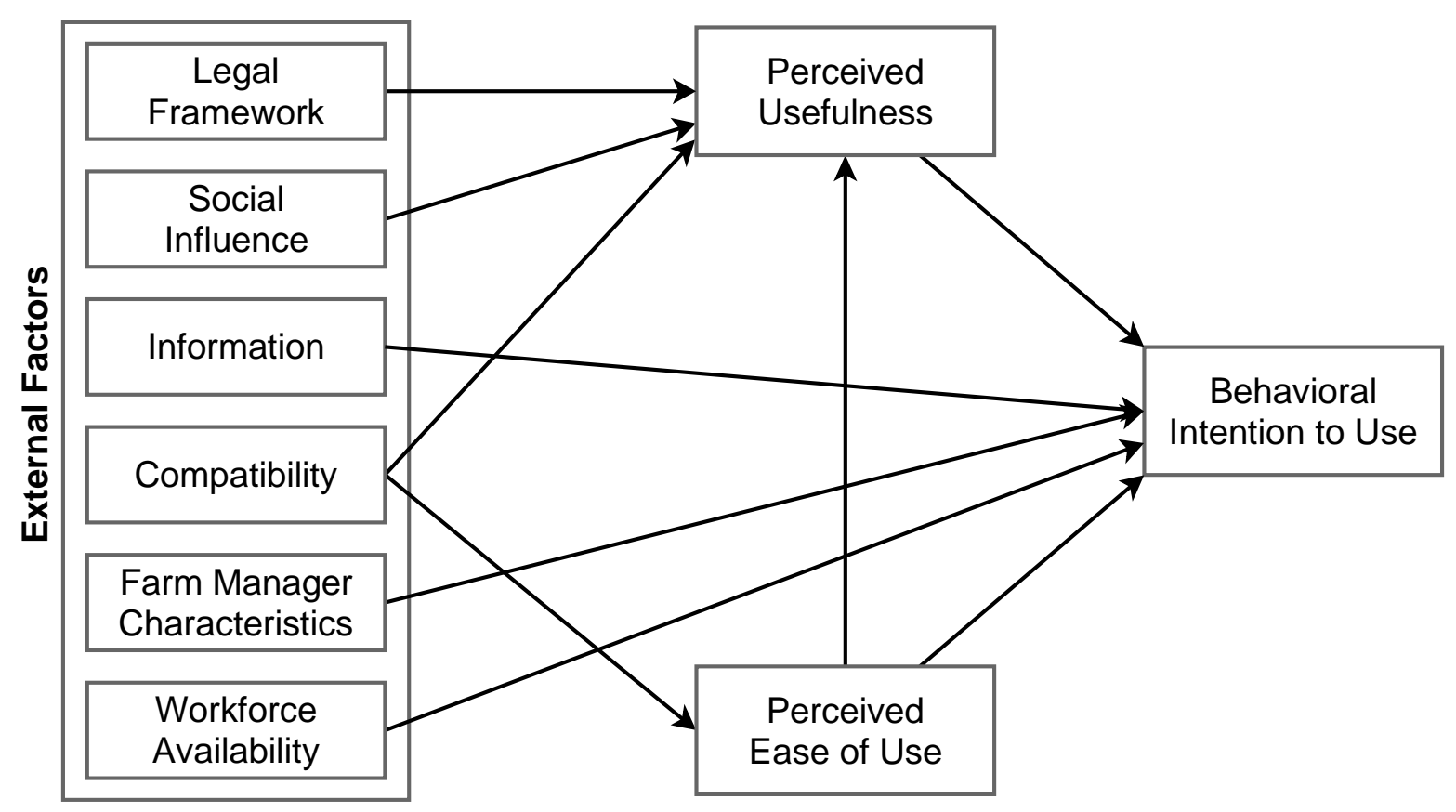

Figure 1: Modified TAM in the context of AFR

Source: Authors' illustration

\subsection{Data Analysis}

A qualitative content analysis by extraction according to (GLÄSER and LAUDEL, 2010; MAYRING, 2015) was used to analyze the data. This was performed in three steps: transcription and data exploration, identification of statements related to the acceptance factors, and summarization and interpretation of the extracted information.

The first step was to transcribe the recorded interviews in full length. The resulting transcripts were made anonymous using the method of factual anonymization. In this process, the extracted data are anonymized to such an extent that links to the individual experts can only be decrypted with a disproportionately high effort (METSCHKE and WELLBROCK, 2002). As a next step, the information relevant to the research objective of this study was extracted from the transcripts and assigned to the TAM factors described above (external factors, perceived usefulness, and perceived ease of use) that served as categories as defined by (MAYRING, 2015). The differentiation of the factors from each other was based on their definitions, anchor examples, and coding rules (see Table A1 in the Appendix). Since there are hardly any studies available for the research object in an agricultural context, the TAM factors were kept open in order to allow the integration of new factors which did not occur in the reviewed literature. Thus, all relevant information of the raw material could be examined under consideration of the interrelations between the factors 
influencing the acceptance of AFR. The methodical procedure was supported by the software "f4-analysis".

\subsection{Sample Description}

The ten selected AgTech startups surveyed for this study are listed in the table below (Table 1). Among these are some of the most promising competitors in this emerging industry, such as the current market leader Naïo Technologies, but also two EU-funded projects aiming to develop a market-ready product. The total number of potential AFR AgTech startups in Europe is probably in the lower two-digit range but is currently difficult to overview. Thus, the experts interviewed are very likely to cover a representative part of the total population of AgTech startups with AFR involvement in Europe.

Agrointelli was founded in 2015 in Denmark, aiming to offer a fully automated farming system for arable farming. As a first step, they developed "Robotti", a versatile and autonomous multiple task robot. AgrOnov, founded in 2015 as a non-profit organization, offers a network to accompany the development of AgTech startups and to promote information and knowledge about their products. The Robert Bosch startup Deepfield Robotics emerged from a research project in 2008 with the publicly funded project "BoniRob", an autonomous robot that can, among other things, measure soil quality and remove weeds. Deepfield Robotics has recently changed its name to "farming revolution" and shifted its focus to mechanical weed control by autonomous robot platforms (FARMING REVOLUTION, 2020). Ecorobotix has been developing solar-powered AFR since 2011, which control weeds with high precision using very small amounts of chemicals. The chemical company BASF is one of its investors (BASF, 2020). K.U.L.T. provides innovative weeding technology for robots and has its roots back in the 1980s. After being temporarily dissolved, they have been newly founded in 2012 under the name "K.U.L.T. Kress Umweltschonende Landtechnik GmbH" and are known for their patented finger weeder. Naïo Technologies is one of the AFR pioneers, founded in 2011. They offer a variety of different AFR for weed control. To date, they have already sold nearly 150 AFR (NAÏO TECHNOLOGIES, 2020). Robotics for Microfarms (ROMI) is an EU-funded project with a duration of 5 years (2017-2022) and a budget of about EUR 4 million. Their aim is to develop open and lightweight robotic platforms for microfarms, helping the farmers to reduce weeds and to monitor their crops (EUROPEAN COMMISSION, 2020a). SITIA is an industrial small- and medium-sized enterprise (SME). In 2019, SITIA launched a hybrid autonomous tractor called "TREKTOR". VineScout has also emerged from an EU-funded project (with a budget of around EUR 2 million), in which a monitoring system (decision support system) embedded in a small and cost-efficient vineyard robot is to be developed to market maturity 
between 2016 and 2020 (EUROPEAN COMMISSION, 2020b). Vitibot was founded in 2016. They developed the AFR "Bakus", a fully electric and autonomous straddle tractor designed to work in the vineyards.

All respondents are directly or indirectly involved in the development process of AFR and were represented as exhibitors at the International Forum of Agricultural Robotics (FIRA) in Toulouse from 8 to 10 December 2019, where the personal interviews took place.

Table 1: Sample description

\begin{tabular}{|c|c|c|c|}
\hline Company & Incorporation & Headquarter & AFR Involvement \\
\hline Agrointelli & 2015 & $\begin{array}{l}\text { Aarhus, Midtjylland } \\
\text { (Denmark) }\end{array}$ & multiple task robot \\
\hline AgrOnov & 2015 & $\begin{array}{c}\text { Bretenière, } \\
\text { Bourgogne-Franche-Comté } \\
\text { (France) }\end{array}$ & AFR consulting \\
\hline Deepfield Robotics & 2008 & $\begin{array}{c}\text { Ludwigsburg, } \\
\text { Baden-Württemberg } \\
\text { (Germany) }\end{array}$ & multiple task robot \\
\hline Ecorobotix & 2011 & $\begin{array}{l}\text { Yverdon-les-Bains, } \\
\text { Waadt (Switzerland) }\end{array}$ & $\begin{array}{l}\text { solar-powered chemical weed } \\
\text { control robot }\end{array}$ \\
\hline K.U.L.T. & 2012 & $\begin{array}{l}\text { Vaihingen an der Enz, } \\
\text { Baden-Württemberg } \\
\text { (Germany) }\end{array}$ & $\begin{array}{l}\text { autonomous mechanical weed } \\
\text { control }\end{array}$ \\
\hline Naïo Technologies & 2011 & $\begin{array}{l}\text { Escalquens, L'Occitanie } \\
\text { (France) }\end{array}$ & weed control robots \\
\hline $\begin{array}{l}\text { RObotics for MI- } \\
\text { crofarms (ROMI) }\end{array}$ & 2017 & $\begin{array}{l}\text { Barcelona, Catalonia } \\
\text { (Spain) }\end{array}$ & multiple task robot \\
\hline SITIA & 1986 & $\begin{array}{l}\text { Bouguenais, Pays de la Loire } \\
\text { (France) }\end{array}$ & multiple task hybrid robot \\
\hline VineScout & 2016 & $\begin{array}{l}\text { Valencia, Valencia } \\
\text { (Spain) }\end{array}$ & monitoring robot (vineyard) \\
\hline VitiBot & 2016 & $\begin{array}{l}\text { Reims, Grand Est } \\
\text { (France) }\end{array}$ & multiple task robot (vineyard) \\
\hline
\end{tabular}

Source: Authors' illustration 


\section{Results}

\subsection{Perceived Usefulness}

All experts agree that AFR must be economically attractive, otherwise one can only: "(...) touch a little, little, little part of the farmers - the technophile."2 (E2). For the majority of the respondents (E2, E3, E4, E5, E6, E8, E10), economic attractiveness is even the most important acceptance factor, because: “(...) a farmer is also an entrepreneur, (...) profitability is the ultimate exclusion criterion." (E4). Consequently, the adoption of AFR by farmers is seen as particularly important in areas where the costs of e.g., weed control (E3), workforce (E4, E5, E7), or time (E9) are relatively high. For expert 8, the price of the robot plays a less important role, since: “(...) the return of investment is probably more important than the cost of the machine and the cost of having the machine or renting the machine just to make sure that in a short period of time you will get back the money." (E8). Expert 9 points out that AFR are expensive to purchase but cheaper to maintain than tractors.

Most of the experts interviewed (E1, E2, E3, E6, E7, E9) also ascribe relevance to the ecological benefits of AFR for the acceptance process among farmers, as another sub-item of perceived usefulness, to the extent that they are forced to deal with resource-saving technologies due to increasing legal restrictions in plant protection. However, one expert sees in this socio-politically driven trend towards a more sustainable agriculture also an opportunity for the development of new technologies: “(...) the current socio-political discussion about reducing pesticides. At the moment, this is a door opener for technologies (...). That's already a big lever at the moment." (E3). With regard to the weighting of this influence, opinions differ. Experts 2, 4, 8 and 9 agree that the ecological benefits are a good sales argument, but not the major aspect. Three other experts differentiate that this aspect is more important for organic farmers than for conventional farmers (E3, E4, E7). For two experts (E5, E10), the ecological advantages are even the most important influencing factor, whereas expert 7 believes that AFR remains a tool and that it always depends on how it is used: "I mean all those robots, it's still tools. So, depends on how you use it. If the idea is trying to reduce the chemicals, robots can be a good tool, (...) if you want to use it in a different way [e.g.,] doing the spraying with more aggressive chemicals, because you don't have the people inside. (...) So, I think it is not the question of the tool, it's what to do with this." (E7). The argument of battery powered AFR concepts is also seen as controversial. For experts 2 and 5, electric engines have an acceptability enhancing effect, as

\footnotetext{
${ }^{2}$ The extracted statements are expressed by direct quotations, where (...) indicates that text has been skipped, while [] indicates that text has been added. This was done for practical reasons.
} 
they are more environmentally friendly and quieter than previous diesel engines. Expert 7 points out that the disposal of batteries is a problem that is often ignored, and for which one has to find a sweet spot. For expert 4, the ecological benefit is: “(...) marketing. Marketing and a big lie, because robots have batteries depending on how they drive. An electric car is not more ecological than when I drive my 20-year-old diesel." (E4).

According to most experts (E2, E3, E4, E5, E6, E8, E10), it is very important for AFR to operate reliably in order to be accepted. For about half of them (E5, E6, E8, E10), safety and reliability are even the second most important acceptance factors after economic efficiency. Experts 2, 3, 6, 7, and 8 see many teething problems with this new technology that need to be eliminated in order to increase its acceptance. Therefore, expert 7 believes that it is important that: "(...) they [farmers] just want to see it working [in the field] and for what I understand they have enough need of this kind of tools to be able to accept" (E7). Two of the respondents (E2, E6) add that farmers want to see that they remain in control and are not completely replaced by AFR. Expert 7 counters that there are still enough tasks that humans can do better than robots and that AFR cannot replace the farmer but only support him.

With regard to perceived usefulness, the relationships assumed at the beginning of the study (Figure 1) are reflected in many ways in the experts' statements. For example, it was emphasized several times that both the ecological and economic benefits of AFR have a direct influence on the behavioral intention to use. In addition, two new relations have been identified. First, the farm structure, as a new external factor, has a direct influence on perceived usefulness as it has a higher value for organic farms. Second, the ecological benefits of AFR appear to be increasing in value as a result of changes in the legal framework in terms of an ongoing ban on pesticides. Thus, the legal framework also has a direct influence on perceived usefulness (see Figure 2).

\subsection{Perceived Ease of Use}

The perceived ease of use was confirmed by most experts as an important acceptance factor (E2, E3, E4, E7, E8, E9), and, for expert 8, it is actually the most important factor for farmers: "But I think for farmers [the most important factor] is the complexity of the machines. (...) it's too difficult to use them." (E8). For this reason, four of the respondents argue that AFR should be designed as simple and clean as possible (E3, E7, E8, E9). Expert 5, on the other hand, sees things completely differently: "If you can use a smartphone, you can use a robot." (E5). Experts 2 and 8 add that it is important to provide farmers with a training program, with expert 7 focusing mainly on practical demonstrations to help 
farmers overcome any fear of dealing with AFR. Even after the purchase of AFR, according to two experts, good support must be guaranteed so that farmers do not have to worry and to: "(...) be afraid of not having the right support at the right time." (E8). Therefore, it would be: “(...) easier for the farmer accept, to take risk.” (E2).

For the TAM factor of perceived ease of use, the assumed influence on the intention of use was reflected in the answers of the respondents, too. The easier AFR are to operate, the more farmers are willing to use them, according to the experts. Hence, the indirect influence of perceived ease of use on perceived usefulness could be confirmed by the proposed training programs; the more farmers are trained about the possible applications of AFR, the more likely they are to recognize a possible benefit or added value of this technology (see Figure 2).

\subsection{Legal Framework}

All experts interviewed agreed on the fact that the legal framework strongly influences the acceptance and speed of adoption of AFR in the agricultural sector. In this context, experts 6,7 , and 10 call for general legislation on the handling of robots before regulating AFR in particular, especially with regard to safety issues: "I think it definitely will cause a problem and we need new legislations for robots in general and especially for agriculture. Especially if it's a person working alongside the robot." (E6) and: "Which is also related to how we can technically ensure that we will not have an accident and this question is quite difficult to solve outdoors.” (E7). So far, the legal framework is relative: “(...) loosely defined what is allowed and what is illegal." (E1), which is why mandatory standards would be helpful, otherwise every manufacturer would try to find a way around it: "So, all the actors of robotics agriculture work together to find some compromise to put some robots on the field." (E5). Due to the perceived lack of governmental support, experts 2, 4, and 8 assume that the diffusion rate of AFR in Europe will slow down, which will give other countries a head start: "[it] is crossing different development rates in different countries. So, we see for example that Japan is releasing faster robots on the same level of development. Whereas Europe and the US are more restrictive, and they are more concerned about safety law. So probably we will see in Japan and Australia first prototypes hitting the market." (E8). In addition to the legal requirements for AFR, three of the interviewees (E3, E7, E8) see a more restrictive legislation for the use of pesticides in agriculture, which could have a positive effect on the acceptance of AFR: "On the other hand, however, the regulations, especially in the area of plant protection, are currently so drastically discussed that people say: 'I need to take the step now, I have to do this, or crops will die'.", (E3). 
The assumed effect of the legal framework on perceived usefulness was unanimously confirmed by the interviewed experts and at the same time, represents the basic requirement for a successful diffusion of this new technology. Not only the legal requirements for the use of AFR but also the tightening legal situation regarding the use of pesticides in agriculture seem to have an impact on acceptance by farmers. In addition, a new relationship of the legal framework to ease of use could be identified, since the sooner legal standards for AFR are introduced, the fewer compromises or legal grey areas have to be used by manufacturers and the easier AFR can be operated (see Figure 2).

\subsection{Social Influence}

Most respondents agree that social influence in the form of sociopolitical pressure has an impact on farmers' decisions (E2, E3, E4, E7, E8, E9), although expert 9 notes that: "It used to be almost nothing, but it is changing really fast. I think the pressure from the government with regulation, but it mostly comes from the end-user. They want something more sustainable, and I think this is pushing really hard on the farmers. So that is definitely something they are considering more and more and it's growing really, really fast." (E9). The discussion about the use of pesticides in agriculture in particular sometimes seems to be driven more by emotion than by fact: "We have conducted many, many interviews and we have had people [farmers] there who were almost close to tears. They say they've had enough." (E3) or: "But it's not always taking into account with very precise technical date, it's more passionate, you know." and: "(...) they [consumers] have this crusade against glyphosate." (E8). According to one respondent (E7), this discussion has an influence on the acceptance of AFR among farmers, even before technical aspects, although in his opinion, the subject of labor safety should not be disregarded. Experts 2, 3, 4, 7, and 8 also see this sociopolitical discussion as a good opportunity for AFR to enter the market in order to meet the demands of society: "If this solution [AFR] can give the farmer some positive recognition it will be more easy [to accept] for the farmer." (E2).

The assumed relationship of social influence on perceived usefulness was clearly confirmed. AFR are seen by farmers as useful tools to counteract social pressure and the associated increasing regulation of pesticide use (see Figure 2).

\subsection{Information}

The vast majority of the respondents (E1, E2, E4, E5, E6, E7, E8, E10) agree that data protection and data autonomy play a role in AFR's acceptance process, of which most consider this role to be very important (E2, E4, E5, E7, E10). Thus, according to experts 
2,4 , and 6, it is important for farmers that: "(...) the data continue to belong to them [farmers] and that they have full control over the data." (E4), because: “(..) they want to be independent." (E2). Several interviewees (E3, E5, E7, E8) see this rather in a twofold way: "On the one hand, [some] say: 'yes, look, this is my field, my production, so to speak, and I would like to keep the pictures with me'. (...) On the other hand (...) they say: 'of course I'm very happy to share this information because I know that the system will then improve continuously, and I will benefit from sharing this information'." (E3), which is also influenced by the type of farm, because in viticulture, for example, exists: "(...) a culture of keeping secrets." (E8) can be important for the success of the company (E7, E8). For one of the interviewees (E4), most farmers do not really understand the term data protection, which is why it is important for AFR's acceptance process to sell the issue to farmers in the right way. Expert 9, on the other hand, sees no influence of the information factor, because: “(...) everyone is working with Google for example or e-mail for store your pictures, so everything is on Google, so it doesn't seem to matter for most of the people. Big companies yes, but small farmers no. Big companies will look at that, but I don't think there is anything to fear." (E9).

It appears that the assumed influence of the factor information on behavioral intention was not just confirmed but also strengthened by the majority of the respondents. Only one expert (E9) saw it in a different light. However, the strength of this effect depends to a large extent on the farm manager characteristics and the farm structure, as the issue of data protection and data autonomy can be more important in viticulture, for example, than in arable farming, where the experts perceive a certain dichotomy among farmers (see Figure 2).

\subsection{Compatibility}

All the respondents agreed that AFR's compatibility with existing technology is very important for the perceived usefulness and ease of use among farmers. Because: "(...) autonomous machines are the natural evolution of farm machinery." (E8) and: “(...) it would be nice if you could combine the past and the future." (E3). Otherwise, it can be confusing for farmers sometimes (E3). According to Expert 6, AFR have to fit into the given farm structures and not vice versa: "I think they [farmers] want to have a robot they can adapt to their own farm. They don't want to do it the other way around. They don't want to adapt their farm to the robot." (E6), whereby this again depends on the farm size: "like small farms that don't have necessarily like machinery and stuff you can adapt with their own habits. Not necessarily with the tractors. So, I think it depends on the farms, but if there is already a tractor it is easier for them to buy a robot, if the robot is adapted to the tractor, I think." (E6). At the same time, three experts (E6, E8, E10) underlined that the existing 
AFR concepts are not yet compatible enough. Two of the respondents (E3, E8) also made it clear that in this process: “(...) nevertheless the core know-how of these companies [established agricultural machinery manufacturers] must be taken along. It is unrealistic to expect that robotics manufacturers will suddenly revolutionize the seed planting process." (E3).

In terms of the TAM factors, the assumed relationships of AFR's compatibility with perceived usefulness and perceived ease of use were confirmed. The better the new technology can be combined with the established technology, the less confusing it is for farmers and the easier it is to use. At the same time, the perceived usefulness of AFR is higher if it can be integrated easily into existing farm structures (see Figure 2).

\subsection{Farm Manager Characteristics}

There were differing views among the respondents on the role of the farmers' age in the acceptance process. Four experts (E1, E4, E6, E9) consider that: “(...) age for example is not something that prevents people from buying a robot." (E1). Expert 9 adds that: "The elderly doesn't like technology most of the time, just like a smartphone they don't like it most of the time. But farmers are used to like technology in general, so I think the age doesn't matter." (E9). Three of the interviewees (E2, E3, E7) share this view insofar as younger people do not necessarily show a higher level of acceptance than older people but are more sensitive or have a greater affinity for technology and that they: “(...) consider that it's more like present. So, like this technology is for today and people a bit older or lower educated tend to think that it's maybe more the future." (E7). On the other hand, experts 4 and 8 attribute an effect on acceptance of age by all means: "Of course, younger farmers tend to be quicker in using such technology. I say, if you plot a curve, acceptance tends to increase with age." (E4). One interviewee also sees an opportunity in AFR: “(...) to have a new better image of agriculture and farming in general for a new and younger generation. I hope it would be that." (E6), in order to counteract the increasing ageing in the sector.

With regard to the educational level of the farmers, the respondents were in agreement. For instance, about half of them (E2, E3, E4, E7, E9) were convinced that there is a correlation between level of education and AFR acceptance. Experts 3 and 8, however, tend to see AFR manufacturers as having a responsibility to design AFR with such simplicity that the level of education should not play a role. Two of the interviewees (E6, E8) find that there is no such influence at all. Instead, acceptance depends more on the farm structure, since: 
“(...) tractors and combines, these machines are already sophisticated, so they [farmers] know how to use them." (E8).

According to the experts, although the risk appetite or curiosity about innovative technologies plays a role in the intention to use AFR, this varies with the personality of the farmer (E3, E4, E6) and is sometimes correlated with the level of education (E1). One of the respondents therefore suggests that leasing concepts should be offered first, so that: "(...) I do not have the entry barriers for the farmer. And then it's relatively easy, then the farmer actually has relatively little risk for the first year and that's the way it has to be." (E3).

\subsection{Workforce Availability}

There is general agreement among the respondents that the workforce availability factor has an influence on the acceptance process. For example, all the experts surveyed see the increasing shortage of labor in agriculture as a driver for AFR's adoption. For two of the interviewees (E1, E7), the lack of skilled workers is even one of the most important drivers of acceptance: "So, they have big issues finding people to drive the tractors and do the job. So, the main problem is this." (E7). In addition, there is an increasing pressure from the trend to use less pesticides, which means more mechanical weed control measures, resulting in more labor-intensive weed control (E7). Three experts (E2, E5, E8) point out that for some physically demanding jobs in agriculture, you need to be in good shape or it is simply unhealthy, which is why you hardly find anyone to do it; therefore, there is no risk of a displacement effect: “(...) nobody wants to do it, because it's very boring and very difficult (...) because we don't have someone. So, for me the robot won't have any impact on the labor market." (E5). Experts 3, 6, 7, and 10, on the other hand, see an effect on the labor market in Eastern Europe, since the use of AFR means that fewer seasonal workers are needed, which could lead to a social problem: "But if you imagine that like $80 \%$ of the labor is replaced by robots it's also a social question in terms of which society we want to build and how we will deal with that." (E7) and: "I think we're playing with new tools and not necessarily thinking about the impacts it's going to have. But we have to think about it of course.” (E6). Two of the respondents (E8, E9) counter that: “(...) a new set of new jobs [is] coming with digital technologies, like data analyzer, like maintenance teams, so there will be jobs." (E8). Finally, one expert points out that: “(...) a person I hire to work in the field for an hour will always be more expensive than a robot." (E4).

The assumed direct influence of workforce availability in the adapted TAM on the behavioral intention to use was clearly confirmed by the need to address labor shortages. In addition, a new relationship was found between workforce availability and perceived 
usefulness, because the less workers are available, the greater the perceived usefulness of AFR for farmers. This relationship is also confirmed by the fact that the removal of the driver reduces the cost of performing the task (see Figure 2).

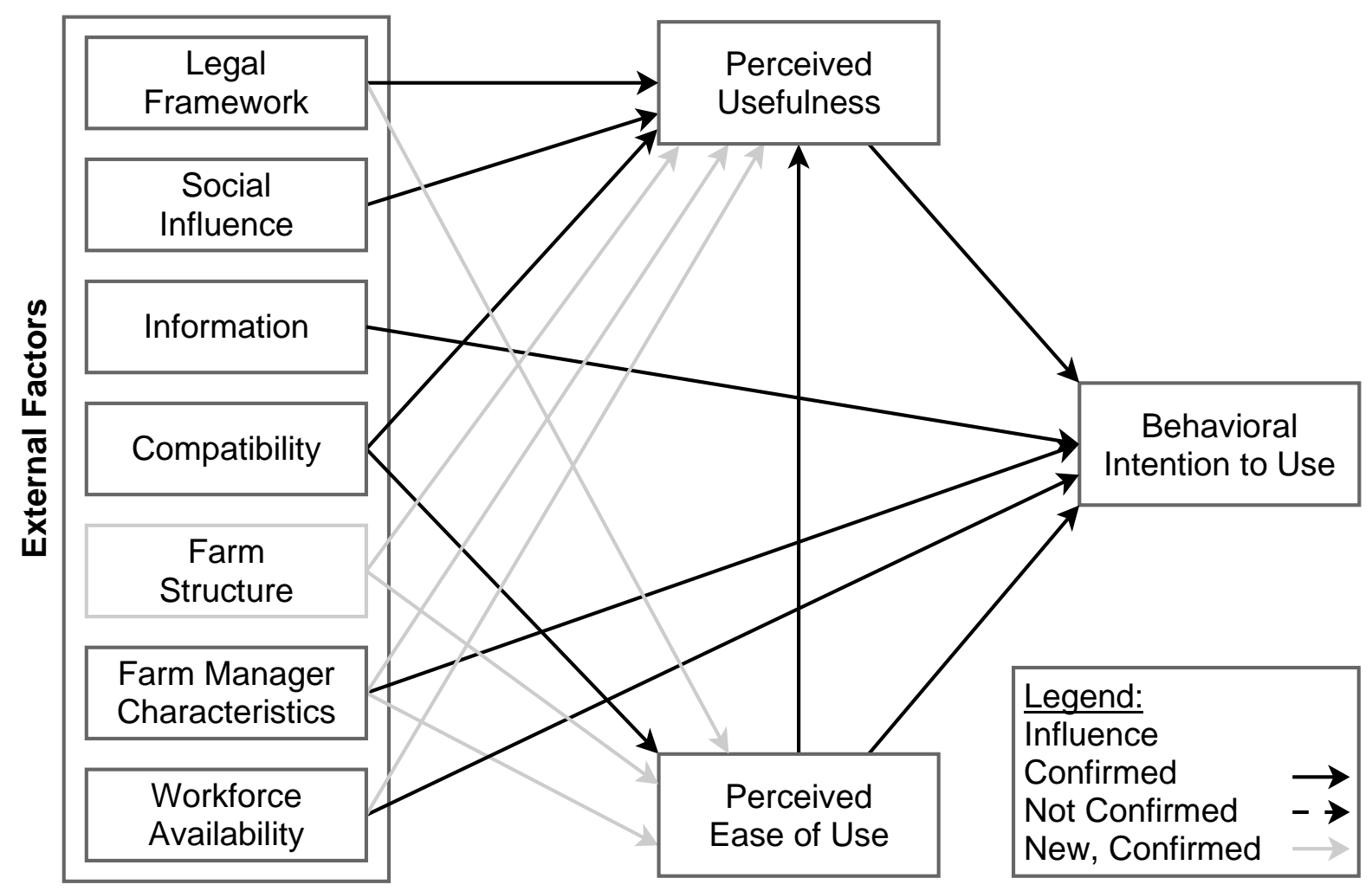

Figure 2: Examined TAM in the context of AFR

Source: Authors' illustration

\section{Discussion and Conclusions}

AFR are considered to be a promising technology to address, at least in part, the many problems in agriculture identified in this study. Nevertheless, there are hardly any studies to date that deal with the multidimensionality of AFR's ex-ante user acceptance process. Such understanding is particularly important, in some cases existentially, for AgTech startups that have committed themselves to this new technology. Therefore, the aim of this explorative paper was to gain a first qualitative understanding of the factors influencing the ex-ante user acceptance and diffusion of AFR from the perspective of AgTech startups to compare the results with the statements of farmers (as buyers) in a follow-up study. For this purpose, guideline-based interviews with ten European AgTech startups dealing with agricultural robotics were conducted at the FIRA in Toulouse (France). The interview guideline was based on the TAM according to DAVIS (1989) and was supplemented by 
external factors (legal framework, social influence, information, compatibility, farm manager characteristics, and workforce (availability)) taken from the scientific literature on this topic.

In conclusion, it was found that due to the scope of the topic, many factors influence the acceptance of farmers from the AgTech startups' point of view. In general, the assumed relationships in the TAM were not only confirmed, but also supplemented by a new factor (farm structure) and several new relationships, which once again demonstrates the transferability of the TAM to various problems of agricultural acceptance research (see also AlAMBAigi and AHANGARI, 2015).

Perceived usefulness was identified by the respondents as the most important or one of the most important factors influencing the ex-ante user acceptance of AFR, with economic benefits ranking ahead of ecological ones. This finding is supported by the results of PIERPAOLI et al. (2013) and SALIMI et al. (2020), who also investigated the ex-ante user acceptance factors of automation and PAT based on a TAM. In this context, the price of AFR plays a role in so far as the return of investment must pay off in the end and not necessarily its absolute amount, which partly confirms the results of RIAL-LOVERA (2018). The author described high investment costs as a possible barrier to acceptance of AFR in a similarly qualitative survey. Therefore, the experts interviewed in this study see AFR first in crops with particularly high labor costs, such as special crops but also row crops, such as sugar beet, before they become economically attractive on a broader scale, which is also confirmed in other studies (LOWENBERG-DeBOER et al., 2020; MinßEN et al., 2015; SHOCKLEY et al., 2019). According to the experts, the ecological advantages of AFR, with the exception of organic farms, have a rather indirect effect on acceptance, as farmers are more or less forced to deal with such technologies due to the progressive reduction of legally permitted pesticide use, which is in line with (DÖRR et al., 2019). At the same time, this sociopolitical pressure is an opportunity for AFR to enter the market more broadly. A similar conclusion was reached by FINGER et al. (2019) as a result of their systematic literature review. They particularly emphasized the importance of the ecological benefits of PAT and the trend towards more sustainable agriculture. Reliability and safety issues of AFR were also considered by most respondents as an important part of perceived usefulness, which is in line with several other studies (BECHAR and VigneAult, 2016; DEVITT, 2018; REDHEAD et al., 2015). Overall, a better communication of the economic but also environmental benefits can contribute to an increase in ex-ante user acceptance, which, regarding the reliability of AFR, should include practical demonstrations. 
The respondents also attributed an important role in the diffusion process to ease of use. This confirms the studies of ADRIAN et al. (2005) and AUBERT et al. (2012) on agriculture and on TAM in general (DAVIS, 1989). In addition, SALIMI et al. (2020) have found that there is a significant positive correlation between automation features such as compatibility, low complexity, reduced workforce needs, etc., and perceived ease of use. According to the experts, AFR should be designed as simply as possible, which is in line with the findings of REDHEAD et al. (2015), according to which too much complexity of AFR can represent an important barrier to its diffusion.

The experts consider the legal framework to be a highly important factor and a major barrier to the diffusion of AFR in the EU. For example, the general operation of AFR is currently considered by both the experts and DÖRR et al. (2019) to be legally uncertain and thus, declared a grey area. DÖRR et al. (2019) also point out that the regulations regarding the use of AFR vary widely around the globe, with Australia, Japan, and the USA being further along than Europe, where scientists tend to register a standstill. In the case of Germany, the federal government has at least stated its intention to establish the legal prerequisites for autonomous vehicles by the end of its term of office, to clarify liability issues (CDU/CSU and SPD, 2018). A proposed solution to liability issues or to clarify the cause of an accident in connection with AFR could be the use of a "black box" analogous to aviation (BMWI, 2013). In addition, the experts emphasized a large, acceptance-influencing effect through the tightening of existing laws affecting agricultural practice; more precisely, by the ever more restrictive regulations on the use of pesticides in agriculture, which are forcing farmers to change. This effect is also confirmed by DöRR et al. (2019), who add that the future market share of autonomous machines will depend largely on regional legislation, while LOWENBERG-DEBOER et al. (2020) add that legal framework may well have an influence on the economic benefits of AFR.

The main opinion of the interviewees that social influence in terms of social pressure for more sustainable farming practices also affects perceived usefulness and thus, indirectly the behavioral intention to use AFR has been confirmed several times in the scientific literature (Foster and RosenZWeig, 1995; VENKATESH and DAVIS, 2000; VenKatesh et al., 2003). ZANDER et al. (2013) conclude that farmers increasingly feel social criticism as a burden and are thus, influenced in their strategic decisions (KUCZERA, 2006). DEVITT (2018) argues that farmers may lose agricultural know-how in the medium as well as long term using AFR and fear a resulting loss of social reputation. This is confirmed by SALIMI et al. (2020), who observed a significant negative correlation between social factors and perceived usefulness of automation adoption in Azerbaijan's agricultural sector. In 
contrast, a consumer survey in the EU showed that only six percent of the respondents would agree to a ban on robots in agriculture (EUROBAROMETER, 2012).

With respect to the influence of collected information by AFR, there is a large consensus among the experts that this is particularly important for the acceptance process among farmers, as on the one hand, their independence is important to them and on the other hand, they want to keep control of the machinery and certain business secrets (e.g., in viticulture). In their media analysis, SCHLEICHER and GRANDORFER (2018) identified data protection and data autonomy as important barriers to acceptance of PAT in agriculture. In contrast, the experts surveyed in a study by RIAL-LOVERA (2018) explicitly did not see data protection and data autonomy as barriers to the diffusion of AFR in agriculture. These contradictory results can possibly be explained by the dependence of the factor information on the characteristics of the farmer and the structure of the farm, as expressed by the experts interviewed in this survey.

The compatibility of AFR was also confirmed in its direct relevance to perceived usefulness and perceived ease of use and its indirect relevance to the behavioral intention to use. This is a particularly important transition phase from existing technologies to digital technologies in order to reach farmers accordingly and not to shy away from the feared complexity of the new system (RIAL-LOVERA, 2018; TRENDOV et al., 2019). This is also confirmed by the results of AUBERT et al. (2012), who conducted a quantitative survey among farmers on PAT acceptance.

The farm structure, as a newly revealed influencing factor, seems to have a direct effect on perceived usefulness, as for example, AFR's ecological added value is higher for organic farms than for conventional farms. On the other hand, new technologies, such as AFR, can be better integrated into existing farm processes if the structural requirements (e.g., the degree of digitization) are met. Thus, DöRR et al. (2019) see the farm structure factor as a possible reason for regional differences in AFR adoption. In addition, SHOCKLEY et al. (2019) see an opportunity for smallholder farms in particular to gain economic benefits over large farms by using more scalable AFR, which is in contrast to the results for technology adoption in general, where larger farms tend to adopt new technology rather than small farms (ANDERSON et al., 1999; BJORNLUND et al., 2009).

The farm manager's characteristics were seen as relevant to the acceptance process, despite some disagreement among experts. Thus, the findings of DEVITT (2018) that better trained and younger farmers are more willing to adopt AFR could only be partially confirmed. While the experts agreed with the influence of educational level, as confirmed by CAFFARO and CAVAllo (2019) for PAT or by DAVIS (1989) in general, they disagreed on the 
influence of age, as AFR should be designed in such a simple way that age is not relevant at all. Again, possible demonstrations of AFR could be helpful in overcoming possible barriers to acceptance due to the personal characteristics of the plant manager.

The availability of the workforce was also confirmed as an important factor influencing AFR's acceptance, the main argument being the existing shortage of skilled workers. This confirms the findings of RIAL-LOVERA (2018), according to which the availability of workforce and rising wages will increase AFR's diffusion in the agricultural sector. The experts interviewed emphasized that there would be no major displacement of existing jobs, as there was already an existing shortage. The situation is different for seasonal workers from low-wage countries. In such countries, GRIEPENTROG (2010) therefore sees the introduction of AFR coming later than in countries with higher wage levels. LOWENBERG-DEBOER et al. (2020) believe that migration flows and the resulting sociopolitical discussion also have an influence in this case. One expert agrees that replacing a driver with an AFR will always be cheaper than driver-operated machines. In addition, the experts see a contrary movement in the sociopolitical demand for a further reduction of pesticides, as this means more mechanical weed control, which in turn is more labor-intensive. However, respondents also noted that AFR will create new jobs, which is in line with DUCKETT et al. (2018), who argue that AFR could attract more highly qualified workers and thus, make agriculture more attractive to young people again.

Since these are empirically collected data, caution and certain limitations must be exercised in interpreting the results with regard to the examined ex-ante user acceptance of AFR. First, the respondents are exclusively AgTech startups with AFR involvement, which may have an overly optimistic view of this new technology and their view on the acceptance of the farmers automatically shows not actual acceptance but their view on it. On the other hand, some of them also gained experience with diffusion barriers and the group of "early adopters" (e.g., Naïo Technologies as the market leader has already sold more than 120 AFR). Thus, a different sample composition could lead to different results (MELENHORST and BouwhuIS, 2004). Second, this is the ex-ante user acceptance of a technology with which most farmers probably have had no contact so far and therefore, little can be said about the actual motivation for a possible adoption. Furthermore, the categorization of the expert statements to the different factors will always remain partly subjective. Causal relationships, as they can be calculated in e.g., quantitative studies using partial least squares (PLS) analysis, cannot be identified in this qualitative paper. Therefore, no hypotheses have been tested, as they cannot be statistically validated in terms of their significance. 
However, the results offer various starting points for further research into the acceptance of autonomous machines in agriculture. Subsequently, the qualitative results must be quantified by surveying the farmers directly to verify or reject the results in a comparison, which is planned in a follow-up study. Furthermore, it would be interesting to investigate the conditions under which farmers would be willing to buy AFR (e.g., by using a choice experiment). Additionally, other stakeholder groups could be included in the analysis, for example, how consumers react to AFR depending on different designs. Furthermore, the results could support political decision-makers in dealing with this new technology (especially with regard to the creation of a contemporary legal framework for AFR) and AFR manufacturers in the promotion of their products among farmers. In conclusion, the findings of this and related studies can provide input for a successful implementation of AFR in agriculture in order to achieve the connected benefits for farmers, but most importantly for society in terms of a more sustainable and efficient farm management and thus, address the various social problems, as listed in the introduction.

\section{Acknowledgement}

We acknowledge support by the Open Access Publication Funds of the Göttingen University.

\section{References}

ADRIAN, A. M., NORWOOD, S. H. and P. L. MASK (2005): Producers' perceptions and attitudes toward precision agriculture technologies. In: Computers and Electronics in Agriculture 48 (3): 256-271, doi: 10.1016/j.compag.2005.04.004.

Alambaigi, A. and I. Ahangari (2015): Technology Acceptance Model (TAM) As a Predictor Model for Explaining Agricultural Experts Behavior in Acceptance of ICT. In: International Journal of Agricultural Management and Development 6 (2): 235-247, doi: 10.22004/ag.econ.262557.

ALT, N. (2018): Gesetzliche Vorgaben für den Feldeinsatz von hochautomatisierten Landmaschinen. In: L. FRERICHS (Ed.): Jahrbuch Agrartechnik 2018. Institute for Mobile Machines and Commercial Vehicles, Brunswick: 1-7.

Anderson, P. D., Wilson, N. P. and D. G. Thompson (1999): Adoption and diffusion of level fields and basins. In: Journal of Agricultural Resource Economics 24 (1): 186-203, doi: 10.22004/ag.econ.30870.

Aubert, B. A., Schroeder, A. and J. Grimaudo (2012): IT as enabler of sustainable farming: An empirical analysis of farmers' adoption decision of precision agriculture technology. In: Decision Support Systems 54: 510-520, doi: 10.1016/j.dss.2012.07.002.

BARTH, S. (1998): Die schriftliche Befragung. URL: https://www.hb.fh-muenster.de/opus4/frontdoor/deliver/ index/docId/489/file/SchriftlicheBefragung.pdf (accessed on 28 November 2020).

BECHAR, A. and C. VignEAUlt (2016): Agricultural robots for field operations: Concepts and components. In: Biosystems Engineering 149: 94-111, doi: 10.1016/j.biosystemseng.2016.06.014. 
Benbasat, I. and A. S. DexTER (1986): An Investigation of the Effectiveness of Color and Graphical Information Presentation under Varying Time Constraints. In: MIS Quarterly 10 (1): 59-83, doi: $10.2307 / 248881$.

BJORNLUND, H., NICOL, L. and K. K. KLEIN (2009): The adoption of improved irrigation technology and management practices - A study of two irrigation districts in Alberta, Canada. In: Agricultural Water Management 96 (1): 121-131, doi: 10.1016/j.agwat.2008.07.009.

BMEL (Bundesministerium für Ernährung und Landwirtschaft) (2020): 2035 Arable Farming Strategy-Prospects for Productive and Diverse Crop Farming. URL: https://www.bmel.de/SharedDocs/ Downloads/EN/Publications/ackerbaustrategieen.pdf?__blob=publicationFile\&v $=6$ (accessed on 6 June 2020).

BMWI (Bundesministerium für Wirtschaft und Technologie) (2013): Recht für funktionale Sicherheit in der Autonomik. URL: https://www.digitale-technologien.de/DT/Redaktion/DE/Downloads/Publika tion/autonomik-Leitfaden2.pdf?_blob=publicationFile\&v=3 (accessed on 29 October 2020).

BukChin, S. and D. KerRet (2018): Food for Hope: The Role of Personal Resources in Farmers' Adoption of Green Technology. In: Sustainability 10 (5): 1615, doi: 10.3390/su10051615.

BurWOOD-TAYLOR, L., LECLERC, R. and M. TILNEY (2016): AgTech investing report: Year in review 2015. AgFunder. URL: https://research.agfunder.com/2015/AgFunder-AgTech-Investing-Report-2015.pdf (accessed on 31 October 2020).

CAFFARO, F. and E. CAVAllo (2019): The Effects of Individual Variables, Farming System Characteristics and Perceived Barriers on Actual Use of Smart Farming Technologies: Evidence from the Piedmont Region, Northwestern Italy. In: Agriculture (MDPI) 9 (5): 111, doi: 10.3390/agriculture9050111.

CDU/CSU and SPD (2018): Koalitionsvertrag zwischen CDU, CSU und SPD. 19. Legislaturperiode. URL: https://www.bundesregierung.de/resource/blob/656734/847984/5b8bc23590d4cb2892b31c987ad67 2b7/2018-03-14-koalitionsvertrag-data.pdf?download=1 (accessed on 1 July 2020).

Chuttur, M. (2009): Overview of the Technology Acceptance Model. Origins, Developments and Future Directions. In: Sprouts: Working Papers on Information Systems 9 (37): 1-21.

DAVIS, F. D. (1989): Perceived Usefulness, Perceived Ease of Use, and User Acceptance of Information Technology. In: MIS Quarterly 13 (3): 319-340, doi: 10.2307/249008.

DevitT, S. K. (2018): Cognitive Factors that Affect the Adoption of Autonomous Agriculture. In: Farm Policy Journal 15 (2): 49-60.

Dörr, J., Fairclough, B., Henningsen, J., Jahić, J., Kersting, S., Menning, P., PePer, C. and F. SCHOlTEN-BusCHHOFF (2019): Scouting the Autonomous Agricultural Machinery Market. Fraunhofer IESE and Kleffmann Group, Kaiserlautern, Lüdinghausen. URL: https://www.iese.fraunhofer.de/content/dam/iese/en/dokumente/smart-farming/Scouting-the-Autonomous-Agricultural-MachineryMarket.pdf (accessed on 31 October 2020).

Duckett, T., Pearson, S., Blackmore, S. and B. Grieve (2018): Agricultural robotics: The future of robotic agriculture. EPSRC UK-RAS Network, London. URL: https://arxiv.org/ftp/arxiv/papers/1806/1806.06762.pdf (accessed on 30 October 2020).

BASF (2020): Ecorobotix Successfully Raises CHF 10.6 Million in Series B Financing with Lead Investors Capagro and BASF Venture Capital. URL: https://www.basf.com/global/de/who-we-are/organization/locations/europe/german-companies/BASF_Venture-Capital/publications/2018/P-18-530.html (accessed on 30 October 2020).

ErL, T., Puttini, R. and Z. Mahmood (2013): Cloud Computing: Concepts, Technology \& Architecture. Prentice Hall, Upper Saddle River.

Eurobarometer (2012): Public Attitudes Towards Robots. Special Eurobarometer 382. URL: https://ec.europa.eu/commfrontoffice/publicopinion/index.cfm/Survey/getSurvey Detail/instrumen687ts/SPECIAL/surveyKy/1044/p/3 (accessed on 1 July 2020). 
EUROPEAN COMMISSION (2020a): RObotics for MIcrofarms. URL: https://cordis.europa.eu/project/id/773875/de (accessed on 1 July 2020).

EUROPEAN COMMISSION (2020b): Intelligent Decision from Vineyard Robots. URL: https://cordis.europa.eu/project/id/737669/de (accessed on 1 July 2020).

FARMING REVOlUTION (2020): Weeding Service for Organic Farms. URL: https://www.farming-revolution.com/ (accessed on 30 October 2020).

FInGER, R., SWINTON, S., El BENNI, N. and E. WALTER (2019): Precision farming at the nexus of agricultural production and the environment. In: Annual Review of Resource Economics 11: 313-335, doi: 10.1146/annurev-resource-100518-093929.

FosteR, A. D. and M. R. RosenZWEIG (1995): Learning by Doing and Learning from Others: Human Capital and Technical Change in Agriculture. In: Journal of Political Economy 103 (6): 1176-1209, doi: $10.1086 / 601447$.

GLÄSER, J. and G. LAUDEL (2010): Experteninterviews und qualitative Inhaltsanalyse als Instrumente. Lehrbuch, fourth edition. VS Verlag für Sozialwissenschaften, Wiesbaden.

Griepentrog, H.W. (2010): Automatisierung in der Außenwirtschaft. In: MOLNAR, C. and M. PIKART-MÜLLER (Eds.): Automatisierung und Roboter in der Landwirtschaft. Kuratorium für Technik und Bauwesen in der Landwirtschaft, Darmstadt: 25-33.

Kester, C., GriePENTROG, H. W., HÖRNER, R. and Z. TUNCER (2013): A survey of future farm automation - a descriptive analysis of survey responses. In: J. V. STAFFORD (Ed.): Precision Agriculture' 13. Wageningen Academic Publishers, Wageningen: 785-792, doi:10.3920/978-90-8686-778-3_97.

KING, A. (2017): Technology: The Future of Agriculture. In: Nature 544 (7651): 21-23, doi: 10.1038/544S21a.

KUCZERA, C. (2006): The influence of the social environment on farm decisions of farmers. Margraf Publishers, Weikersheim: 56-171.

Lowenberg-DeBoer, J., HuAng, I. Y., Grigoriadis, V. and S. BlackMORE (2020): Economics of robots and automation in field crop production. In: Precision Agriculture 21 (2020), doi: 10.1007/s11119-01909667-5.

MAYRING, P. (2015): Qualitative Inhaltsanalyse: Grundlagen und Techniken. Beltz, Weinheim.

MELENHORST, A.-S. and D. G. Bouwhuis (2004): When do older adults consider the internet? An exploratory study of benefit perception. In: Gerontechnology 3: 89-101, doi: 10.4017/gt.2004.03.02.004.00.

MetsChKe, R. and R. WeLlBRoCK (2002): Datenschutz in Wissenschaft und Forschung. Druckerei Conrad $\mathrm{GmbH}$, Berlin.

MiLLER, L. H. (1977): A Study in Man-Machine Interaction. In: Association fOR COMPUTING MACHINERY (Ed.): Proceedings of the National Computer Conference, Dallas, Texas, USA, 13-16 June 1977: 409421, doi: 10.1145/1499402.1499475.

Minßen, T.-F., Urso, L.-M., GAus, C.-C. and L. Frerichs (2015): Mit autonomen Landmaschinen zu neuen Pflanzenbausystemen. In: ATZoffhighway 8 (3): 6-11, doi: 10.1007/s35746-015-0028-y.

NAÏO TeChNOlogies (2020): La Robotique Au Service de l'Agriculture. URL: https://www.naio-technologies.com/ (accessed on 1 July 2020).

PATHAK, H. S., BROWN, P. and T. BEST (2019): A systematic literature review of the factors affecting the precision agriculture adoption process. In: Precision Agriculture 13 (9): 1-25, doi: 10.1007/s11119-01909653-x.

Paustian, M. and L. THeuvsen (2016): Adoption of precision agriculture technologies by German crop farmers. In: Precision Agriculture 18 (5): 701-716, doi: 10.1007/s11119-016-9482-5.

Pierpaoli, E., Carli, G., PignatTi, E. and M. Canavari (2013): Drivers of Precision Agriculture Technologies Adoption: A Literature Review. In: Procedia Technology 8: 61-69, doi: 10.1016/j.protcy.2013.11.010. 
Redhead, F., Snow, S., Vyas, D., Bawden, O., Russell, R., Perez, T. and M. Brereton (2015): Bringing the Farmer Perspective to Agricultural Robots. In: BEgOLE, B., KIM, J., INKPEN, K. and W. WOO (Eds.): Proceedings of the 33rd Annual ACM Conference Extended Abstracts on Human Factors in Computing Systems. Association for Computing Machinery, New York: 1067-1072, doi: 10.1145/2702613.2732894.

Rial-LoverA, K. (2018): Agricultural Robots: Drivers, Barriers and Opportunities for Adoption. In: N. Tremblay (Ed.): Proceedings of the 14th International Conference on Precision Agriculture, Montreal, 24-27 June 2018. The International Society of Precision Agriculture, Monticello Illinois.

Rogers, E. M. (1995): Diffusion of Innovations. Free Press, New York.

Salimi, M., Pourdarbani, R. and B. A. NOURI (2020): Factors Affecting the Adoption of Agricultural Automation Using Davis's Acceptance Model (Case Study: Ardabil). In: Acta Technologica Agriculturae 23 (1): 30-39, doi: 10.2478/ata-2020-0006.

SCHLEICHER, S. and M. GRANDORFER (2018): Digitalisierung in der Landwirtschaft: Eine Analyse der Akzeptanzhemmnisse. In: RuCKelshausen, A., MeYer-Aurich, A., BOrCHARDt, K., HOfaCKer, C., Loy, J.-P., Schwerdtfeger, R., Sundermeier, H.-H., Floto, H. and B. Theuvsen (Eds.): Digitale Marktplätze und Plattformen. Lecture Notes in Informatics 278, Gesellschaft für Informatik, Bonn: 203-206.

SHOCKLEY, J. M., Dillon, C. R. and S. A. SHEARER (2019): An economic feasibility assessment of autonomous field machinery in grain crop production. In: Precision Agriculture 20 (2019): 1068-1085, doi: 10.1007/s11119-019-09638-w.

Trendov, N. M., VAras, S. and M. ZenF (2019): Digital Technologies in Agriculture and Rural Areas: Status Report. Food and Agricultural Organization of the United Nations, Rome: 118-120.

Venkatesh, V. and F. D. DAvis (2000): A Theoretical Extension of the Technology Acceptance Model: Four Longitudinal Field Studies. In: Management Science 46 (2): 186-204, doi: 10.1287/mnsc.46.2.186.11926.

Venkatesh, V., Morris, M. G., Davis, G. B. and F. D. DAvis (2003): User Acceptance of Information Technology: Toward a Unified View. In: MIS Quarterly 27 (3): 425-478, doi: 10.2307/30036540.

VERMA, P. and N. SINHA (2018): Integrating perceived economic wellbeing to technology acceptance model: The case of mobile based agricultural extension service. In: Technological Forecasting and Social Change 126: 207-216, doi: 10.1016/j.techfore.2017.08.013.

Zander, K., Isermeyer, F., BÜrgelt, D., Christoph-Schulz, I. B., SAlamon, P. and D. Weible (2013): Erwartungen der Gesellschaft an die Landwirtschaft. Stiftung Westfälische Landwirtschaft, Münster. 


\section{Appendix}

\section{Interview Guideline}

1. Do you agree that the interview may be recorded and used in anonymous form for my research?

2. What role will autonomous field robots play in agriculture over the next ten years?

3. In your opinion, which factors inhibit/promote the acceptance of autonomous field robots among farmers?

4. What influence do you attribute to the farm manager's age, educational level and risk propensity?

5. Do you consider the aspect of data protection and data sovereignty to be important for the acceptance of autonomous field robots among farmers?

6. In which areas of agriculture can you imagine the use of autonomous field robots?

7. How important do you think it is that autonomous field robots are compatible with different tools and possibly conventional equipment?

8. Do you expect the introduction of autonomous field robots to have an impact on the distribution structure in the agricultural machinery market?

9. What effects do you see on the agricultural labor market if autonomous field robots are used as standard?

10. How do you assess the ecological added value as an influencing factor on the acceptance among farmers?

11. Can conflicts with current legislation be a problem for the introduction of autonomous field robots in agriculture?

12. In summary, which factors do you think have the greatest influence on the adoption of autonomous robots in agriculture?

13. When do you think the commercial use of autonomous field robots in agriculture will start?

14. How much will the robots cost? 
Table A1: Coding Guide

\begin{tabular}{|c|c|c|c|}
\hline Factor & Definition & Anchor Example & Coding Rule \\
\hline $\begin{array}{l}\text { Perceived } \\
\text { Usefulness }\end{array}$ & $\begin{array}{l}\text { - Economic efficiency (increase } \\
\text { in yield or profit; input savings; } \\
\text { investment costs) } \\
\text { - Ecological sustainability } \\
\text { - Reliability (reliable operation } \\
\text { and performance during work } \\
\text { peaks) } \\
\text { - Reduced workload }\end{array}$ & $\begin{array}{l}\text { "(...) and that the farm is } \\
\text { economically and ecologi- } \\
\text { cally in good shape." (E3) }\end{array}$ & $\begin{array}{l}\text { Statements on prof- } \\
\text { itability, ecological } \\
\text { added value, func- } \\
\text { tionality, and per- } \\
\text { formance expecta- } \\
\text { tions. }\end{array}$ \\
\hline $\begin{array}{l}\text { Perceived } \\
\text { Ease of Use }\end{array}$ & $\begin{array}{l}\text { - Handling/user interface } \\
\text { - Own maintenance }\end{array}$ & $\begin{array}{l}\text { "The technology has to be } \\
\text { simple anyway." (E9) }\end{array}$ & $\begin{array}{l}\text { Intensity of per- } \\
\text { ceived ease of use's } \\
\text { effect on ac- } \\
\text { ceptance. }\end{array}$ \\
\hline $\begin{array}{l}\text { Legal } \\
\text { Framework }\end{array}$ & $\begin{array}{l}\text { - Conflicts with law/liability (in } \\
\text { the fields and on public roads) } \\
\text { - legal restrictions on substitutes } \\
\text { (e.g., for pesticides) } \\
\text { - Subsidies }\end{array}$ & $\begin{array}{l}\text { "Yes, certainly. Because you } \\
\text { have to adapt the law (...)" } \\
\text { (E10) }\end{array}$ & $\begin{array}{l}\text { Legal framework as } \\
\text { a requirement and } \\
\text { driver for the adop- } \\
\text { tion of AFR. }\end{array}$ \\
\hline $\begin{array}{l}\text { Social } \\
\text { Influence }\end{array}$ & $\begin{array}{l}\text { - Influence of social networks } \\
\text { (e.g., society, consultants, col- } \\
\text { leagues) }\end{array}$ & $\begin{array}{l}\text { "They [society] want some- } \\
\text { thing more sustainable and I } \\
\text { think this is pushing really } \\
\text { hard on the farmers." (E9) }\end{array}$ & $\begin{array}{l}\text { Statements on the } \\
\text { influence of social } \\
\text { networks on the de- } \\
\text { cision-making pro- } \\
\text { cess of farmers. }\end{array}$ \\
\hline Information & $\begin{array}{l}\text { - Data processing and use of } \\
\text { farmers actions for strategic } \\
\text { production planning } \\
\text { - Data protection and data auton- } \\
\text { omy }\end{array}$ & $\begin{array}{l}\text { "(...) everyone is working } \\
\text { with Google (...) it doesn't } \\
\text { seem to matter for most of } \\
\text { the people. Big companies } \\
\text { yes, but small farmers no." } \\
\text { (E9) }\end{array}$ & $\begin{array}{l}\text { Intensity of the in- } \\
\text { formation effect on } \\
\text { acceptance. }\end{array}$ \\
\hline Compatibility & $\begin{array}{l}\text { - Cooperation with existing tech- } \\
\text { nology }\end{array}$ & $\begin{array}{l}\text { "(...) a robot will be more } \\
\text { important if it is connectable } \\
\text { with all the other machines, } \\
(\ldots) ”(\mathrm{E} 2)\end{array}$ & $\begin{array}{l}\text { Intensity of the } \\
\text { compatibility effect } \\
\text { on acceptance. }\end{array}$ \\
\hline $\begin{array}{l}\text { Farm Manager } \\
\text { Characteristics }\end{array}$ & $\begin{array}{l}\text { - Age, education, and risk appe- } \\
\text { tite }\end{array}$ & $\begin{array}{l}\text { "(...) the farmers of today } \\
\text { were not born with these } \\
\text { technologies. (...) So yes it's } \\
\text { preventing the use of digital } \\
\text { technologies." (E6) }\end{array}$ & $\begin{array}{l}\text { Classification by } \\
\text { age, educational } \\
\text { level and risk appe- } \\
\text { tite. Intensity of the } \\
\text { effect on ac- } \\
\text { ceptance. }\end{array}$ \\
\hline $\begin{array}{l}\text { Workforce } \\
\text { Availability }\end{array}$ & $\begin{array}{l}\text { - Influence of workforce issues } \\
\text { (especially availability of } \\
\text { workforce) }\end{array}$ & $\begin{array}{l}\text { "Regulations are one ques- } \\
\text { tions, but also safety on the } \\
\text { work and the availability of } \\
\text { workforce." (E7) }\end{array}$ & $\begin{array}{l}\text { Intensity of the } \\
\text { workforce availabil- } \\
\text { ity effect on ac- } \\
\text { ceptance. }\end{array}$ \\
\hline
\end{tabular}

\section{Source: Authors' illustration}




\section{Teil III:}

\section{Strukturwandel und dezentrale Energieversorgung in der deutschen Landwirtschaft}

\section{III.1 Die gesellschaftliche Wahrnehmung von bäuerlicher und industrieller Landwirtschaft}

Friedrich Rübcke von Veltheim, Christian Schaper und Heinke Heise

Geleisteter Eigenanteil an dem Beitrag

\begin{tabular}{llllll}
\hline $\begin{array}{l}\text { Literatur- } \\
\text { recherche }\end{array}$ & $\begin{array}{l}\text { Entwicklung } \\
\text { des } \\
\text { Forschungs- } \\
\text { rahmens }\end{array}$ & $\begin{array}{l}\text { Konzept und } \\
\text { Design der Stu- } \\
\text { die }\end{array}$ & $\begin{array}{l}\text { Auswahl, Aus- } \\
\text { führung und } \\
\text { Entwicklung } \\
\text { von Methoden }\end{array}$ & $\begin{array}{l}\text { Interpretation } \\
\text { der } \\
\text { Ergebnisse }\end{array}$ & $\begin{array}{l}\text { Konzept und } \\
\text { Schreiben des } \\
\text { Artikels }\end{array}$ \\
\hline$>60 \%$ & $50 \%$ & $50 \%$ & $50 \%$ & $>60 \%$ & $>75 \%$ \\
\hline
\end{tabular}

Dieser Beitrag ist so oder in ähnlicher Fassung veröffentlicht in der wissenschaftlichen Zeitschrift „Austrian Journal of Agricultural Economics and Rural Studies “ 28 (22). 


\section{Inhalt}

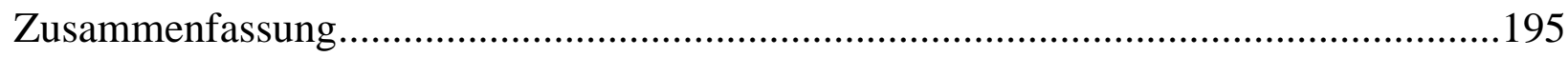

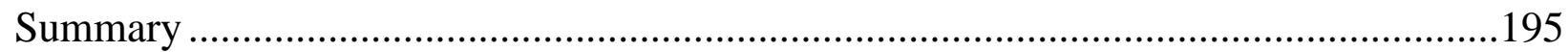

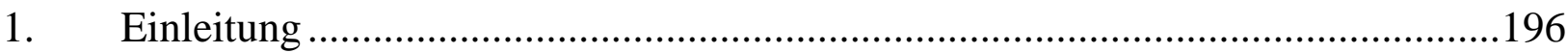

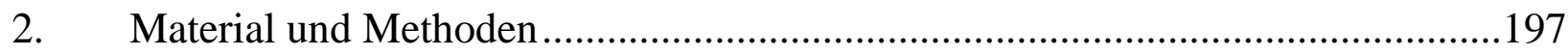

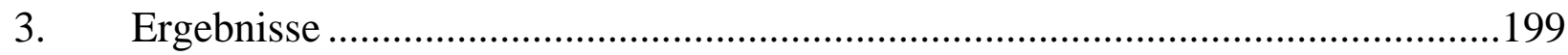

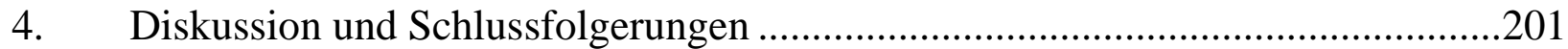

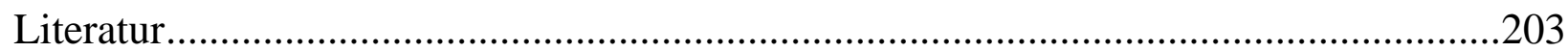




\section{Zusammenfassung}

Die vorliegende Studie untersucht die gesellschaftliche Wahrnehmung der Begriffe ,industrielle“ und „bäuerliche“ Landwirtschaft. Dafür mussten 1.150 Verbraucher im Zuge einer standardisierten Online-Befragung verschiedene Schlagworte hinsichtlich ihrer Assoziation mit einer ,,industriellen“ oder einer „bäuerlichen“ Landwirtschaft bewerten. Dabei wurde sehr deutlich, dass die Bewertungen überwiegend konträr verlaufen und die befragten Verbraucher einer ,industrialisierten“ Landwirtschaft eher negativ besetzte Begriffe bzw. Aussagen, wie „Massentierhaltung“ oder „starker Verdrängungswettbewerb nach dem Motto: „Wachsen oder Weichen“, zuordnen. Die größte Uneinigkeit im Antwortverhalten bestand für die ,industrialisierte“ Landwirtschaft bei der Zuordnung von Schlagworten bzw. Kurzaussagen wie „Verantwortung für Boden, Pflanzen und Natur“ oder „Landwirtschaftlicher Familienbetrieb“; die größte Einigkeit bezogen auf die „,äuerliche“ Landwirtschaft bei Begriffen bzw. Aussagen wie „Landleben“ oder „Verbundenheit mit Hof, Natur und Heimat".

\section{Schlagworte}

Bäuerlich, Befragung, Industriell, Landwirtschaft, Verbraucher

\section{Summary}

This study examines the social perception of the terms "industrial" and "rural" agriculture. Therefore, 1.150 consumers were confronted with different keywords in the course of a standardized online survey to evaluate how much they associate the given terms with an "industrial" or "rural" agriculture. It turned out that the evaluations were predominantly contrary and that the consumers surveyed assigned rather negative terms to "industrialized" agriculture, such as "factory farming" or "strong predatory competition based on the motto: growing or giving way". The greatest agreement in response was - in case of "rural" agriculture - in terms or statements such as "country life" or "connection with farm, nature and homeland".

\section{Keywords}

agriculture, consumer, industrial, rural, survey 


\section{Einleitung}

Mit einer unter hohem Preisdruck stehenden und durch zunehmende Intensivierung und Spezialisierung gekennzeichneten Landwirtschaft gingen nicht nur Produktivitätsfortschritte und Arbeitserleichterungen einher. Der Fortschritt rief auch eine steigende gesellschaftliche Kritik an ebensolchen Produktionsmethoden hervor (BMEL, 2018; BUSCH et al., 2013; JANSEN und VELLEMA, 2004; WBA, 2015). So herrscht laut KANTAR EMNID (2017) mit der konventionellen landwirtschaftlichen Produktion eine allgemeine Unzufriedenheit, die in urbanen Gegenden stärker ausfällt als in ländlichen Regionen und die Agrar- und die Lebensmittelindustrie in den Mittelpunkt der gesellschaftlichen Diskussion gebracht hat (ISERMEYER, 2014; WBA, 2015). Im Zuge dieser wird gerne von einer wachsenden „Industrialisierung der Landwirtschaft“ gesprochen (KAYSER et al., 2012; SPILLER et al., 2012;). Dabei werden unerwünschte Nebenwirkungen auf Umwelt, Tier und Mensch beklagt und eine Rückbesinnung auf eine „bäuerliche“ Landwirtschaft gefordert (BULLER und MORRIS, 2004; CAMPBELl, 2009; GERKE, 2017). Galt diese ehemals noch als rückwärtsgewandt und unvorteilhaft, ist der Begriff einer „bäuerlichen” Landwirtschaft heute mehr denn je en vogue und wird in zunehmendem Maße positiv besetzt (BIRNER, 2012; KREMEN et al., 2012; ZANDER et al., 2013).

In der agrarpolitischen und gesellschaftlichen Diskussion der vergangenen Jahre, die durch einen Strukturwandel in der Landwirtschaft - v.a. bezogen auf Betriebs- und BestandsgröBen bestimmt wird, tauchen diese beiden Begriffe immer häufiger auf (KAYSER et al., 2012; WoodHouse, 2010). Der Strukturwandel zwingt Landwirte mit kosteneffizienter produzierenden Betrieben Schritt zu halten, da diese sonst Gefahr laufen, unrentabel zu werden und schließlich aus der Produktion aussteigen müssen. BALMANN et al. (2016) umschreiben dieses Phänomen mit dem Bild einer „landwirtschaftlichen Tretmühle“, die nie ruht und durch Technologieschübe dafür sorgt, dass die Produktion steigt und die Erzeugerpreise sinken. Je globaler ein Markt ist, desto schneller dreht sich die Tretmühle und desto mehr unrentabel gewordene Betriebe lösen sich auf. Dieser Vorgang stößt auf zunehmenden Widerstand seitens der Gesellschaft, der sich in politischen Debatten und Entscheidungen niederschlägt - etwa in Form der Diskussion um die Kappung der Direktzahlungen bei größeren Betrieben (BÖHME, 2011; BREUSTEDT, 2013). Die dabei immer wieder auftretenden Begriffe einer ,industriellen“ und einer „,bäuerlichen“ Landwirtschaft unterscheiden sich in Art und Umfang der Verwendung beachtlich (ABL, 2015; AGRARBÜNDNIS, 2001; EDELMANN, 2013; EKD, 2017).

Eine einheitliche Verwendung, geschweige denn eine fassbare Definition dieser Begrifflichkeiten sucht man in Deutschland mitunter vergebens. Ziel dieses Beitrages ist es daher, 
sich den beiden Begriffen ,industriell“ oder „bäuerlich“, bezogen auf die Landwirtschaft, zunächst aus Sicht deutscher Konsument mithilfe einer quantitativen Befragung anzunähern und somit weiter zu konkretisieren. Wie nimmt die Gesellschaft in Deutschland diese Begriffe wahr und werden sie tatsächlich als gegensätzlich empfunden oder existieren möglicherweise Überschneidungen? Sind diese Begriffe erst fassbarer geworden, könnten in aufbauenden Studien hieraus Lösungsansätze für die offenkundigen Akzeptanzprobleme einer konventionell wirtschaftenden Landwirtschaft erwachsen.

Nach einer kurzen Beschreibung des verwendeten Materials und der angewandten Analysemethoden, folgt in Kapitel drei die Ergebnisvorstellung. In Kapitel vier werden die Ergebnisse kurz diskutiert, bevor dieser Beitrag seinen Abschluss in verschiedenen Schlussfolgerungen findet.

\section{Material und Methoden}

Für die Studie wurde im Oktober 2017 eine standardisierte Online-Befragung durchgeführt, die sich hinsichtlich der Zusammensetzung an der deutschen Bevölkerung mit Bezug auf die Geschlechterverteilung, die Altersklassen und die Verteilung auf die Bundesländer orientiert, um auf diese Weise eine möglichst hohe Repräsentativität zu gewährleisten. Die Befragung beschäftigt sich mit dem Verständnis deutscher Verbraucher von den beiden Begriffen einer „,bäuerlichen“ und ,industriellen“ Landwirtschaft. Von den befragten Probanden beendeten 1.150 Verbraucher die Umfrage vollständig. Die Fragen zur Assoziation der 21 Schlagworte bzw. Kurzaussagen zu Landwirtschaft mussten von den Verbrauchern den Begriffen „,bäuerlich“ oder „,industriell“ mittels fünfstufiger Likert Skalen (1 = „Trifft gar nicht zu“ bis $5=$, Trifft voll und ganz zu“) zugeordnet werden, um ein besseres Verständnis davon zu bekommen, über welche Begriffe die befragten Verbraucher ,bäuerliche“ und „industrielle“ Landwirtschaft definieren. Die genauen Fragestellungen lauteten: „,Mit welchen Schlagworten verbinden Sie „, bäuerliche Landwirtschaft“? Bitte kreuzen Sie Zutreffendes an. “ und ,Im Gegenzug möchten wir jetzt von Ihnen wissen, was Sie mit ,, industrieller Landwirtschaft" verbinden (bitte kreuzen Sie Zutreffendes an). “. Die Auswahl der Schlagworte war das Resultat einer der Studie vorgeschalteten, umfassenden Literaturrecherche (BöHME, 2014; EdELMANN, 2013; LAMINE, 2015; ZANDER et al., 2013). Die Antworten der Verbraucher wurden deskriptiv mit dem Statistikprogramm „IBM SPSS Statistics - Version 24“" ausgewertet. Zudem wurden mithilfe von T-Tests die Mittelwerte der Aussagen verglichen, um festzustellen, ob die ermittelten Differenzen zwischen den verschiedenen Schlagworten signifikant sind. 
Die genaue Zusammensetzung der Stichprobe sowie die relative Verteilung der deutschen Bevölkerung (laut Statistischem Bundesamt) sind in der Tabelle 1 dargestellt (DESTATIS, 2016). Die Stichprobe entspricht hinsichtlich der Geschlechterverteilung, der Altersklassen und der Verteilung auf die Bundesländer dem deutschen Durchschnitt. Darüber hinaus sind die Daten repräsentativ hinsichtlich des Schulabschlusses, der Verteilung der Wohnorte der Befragten zwischen urbanem und ruralem Lebensraum sowie des monatlichen Nettoeinkommens. Die Bundesländer wurden der Übersichtlichkeit halber in der Tabelle 1 in die vier Regionen Nord (Bremen, Hamburg, Mecklenburg-Vorpommern, Niedersachsen und Schleswig-Holstein), Süd (Baden-Württemberg und Bayern), West (Hessen, Nordrhein-Westfalen, Rheinland-Pfalz und das Saarland) sowie Ost (Berlin, Brandenburg, Sachsen, Sachsen-Anhalt und Thüringen) unterteilt.

Tabelle 1: Soziodemografische Charakteristika der Stichprobe verglichen mit der deutschen Grundgesamtheit an Verbraucher

Ausprägung Deutsche Grundgesamtheit $\quad$ Stichprobe $(n=1.150)$

\begin{tabular}{lrcr}
\hline Geschlecht & männlich & $49 \%$ & $49 \%$ \\
\hline weiblich & $51 \%$ & $51 \%$ \\
\hline Herkunft & $16-35$ & $23 \%$ & $20 \%$ \\
& $36-55$ & $33 \%$ & $33 \%$ \\
& $>56$ & $44 \%$ & $47 \%$ \\
\hline Einkommen & Nord & $18 \%$ & $17 \%$ \\
& Weid & $29 \%$ & $27 \%$ \\
& Ost & $35 \%$ & $36 \%$ \\
& $<900 €$ & $18 \%$ & $12 \%$ \\
\hline Abschluss & $901-1.500 €$ & $11 \%$ & $11 \%$ \\
& $1.501-3.200 €$ & $20 \%$ & $21 \%$ \\
& $>3.200 €$ & $43 \%$ & $51 \%$ \\
& (noch) ohne & $27 \%$ & $18 \%$ \\
\hline Hauptschule & $8 \%$ & $5 \%$ \\
& Mittlere Reife & $33 \%$ & $32 \%$ \\
& Abitur & $29 \%$ & $28 \%$ \\
\hline
\end{tabular}

Quelle: Eigene Berechnung nach DESTATIS (2016) 


\section{Ergebnisse}

Die Ergebnisse dieser Umfrage mitsamt der unterzogenen Analyse sind ausschnittsweise in Abbildung 1 und 2 dargestellt. Dabei konzentriert sich die Abbildung 1 auf die Schlagworte, welche eher mit einer „bäuerlichen“ als mit einer ,industriellen“ Landwirtschaft verbunden wurden. In Abbildung 2 verhält es genau entgegengesetzt. Die Abbildungen verdeutlichen, dass die Bewertungen der verschiedenen Schlagworte hinsichtlich ihrer Assoziation zur „,bäuerlichen“ und ,,industriellen“ Landwirtschaft überwiegend konträr verlaufen.

Die „bäuerliche“ Landwirtschaft wurde vor allem mit den Schlagworten „Verantwortung für Tiere, Boden, Pflanzen und Natur“ $(\mu=1,7)$, ,Landleben“ $(\mu=1,7)$, ,Verbundenheit mit Hof, Natur und Heimat" $(\mu=1,7)$ und ,, selbstverantwortliches Arbeiten des/r Landwirts/in“ ( $\mu=1,7)$ in Verbindung gebracht. Daneben wurden auch die Schlagworte ,, landwirtschaftlicher Familienbetrieb“ $(\mu=1,9)$ und ,, respektvoller Umgang mit Boden, Pflanzen, Tieren, Natur und Menschen “ $(\mu=1,9)$ mit der ,bäuerlichen“ Landwirtschaft assoziiert. Ihr werden darüber hinaus die Schlagworte ,, artgerechte Tierhaltung “ $(\mu=2,0)$, ,Umsetzung von Tierwohlmaßnahmen “ $(\mu=2,1)$, ,Pflege und Erhalt der Kulturlandschaft “ ( $\mu$ = 2,1), ,überschaubare Betriebsgröße“ $(\mu=2,1)$, ,umweltschonende Wirtschaftsweise “ $(\mu=2,2)$ und ,, Erzielung eines angemessenen Einkommens für den Lebensunterhalt sowie den Erhalt von Arbeitsplatz und Hof" $(\mu=2,2)$ zugeordnet.

Dagegen wird die ,industrielle“ Landwirtschaft durch die Schlagworte „Einsatz modernster Technik“ $(\mu=1,7)$, ,Massentierhaltung “ $(\mu=2,0)$, , starker Verdrängungswettbewerb nach dem Motto: Wachsen oder Weichen“ $(\mu=2,0)$, ,schnelles Betriebswachstum“ $(\mu=2,0)$ und ,Erzielung einer hohen Rendite auf das eingesetzte Eigenkapital “ $(\mu=2,0)$ gekennzeichnet. Befragte sehen eine Verbindung mit den Schlagworten „,der Betrieb beschäftigt zahlreiche familienfremde Mitarbeiter “ $(\mu=2,1)$ sowie „Beteiligung außerlandwirtschaftlicher Investoren am Kapital des Betriebes “ $(\mu=2,1)$. Demgegenüber ist eine leicht ablehnende Tendenz hinsichtlich der Schlagworte „überschaubare Betriebsgröße“ $(\mu=3,5)$, , landwirtschaftlicher Familienbetrieb “ $(\mu=3,6)$ und ,es arbeiten nur der/die Betriebsleiter/in und seine/ihre Familie auf dem Betrieb “ $(\mu=3,7) \mathrm{zu}$ beobachten. Der TTest ergab in beiden Fällen, dass sich die Bewertungen aller Schlagworte hinsichtlich ihrer Mittelwerte zwischen der ,bäuerlichen“ und der ,industriellen“ Landwirtschaft hoch $(\mathrm{p} \leq 0,01)$ bis höchst $(\mathrm{p} \leq 0,001)$ signifikant unterscheiden. 


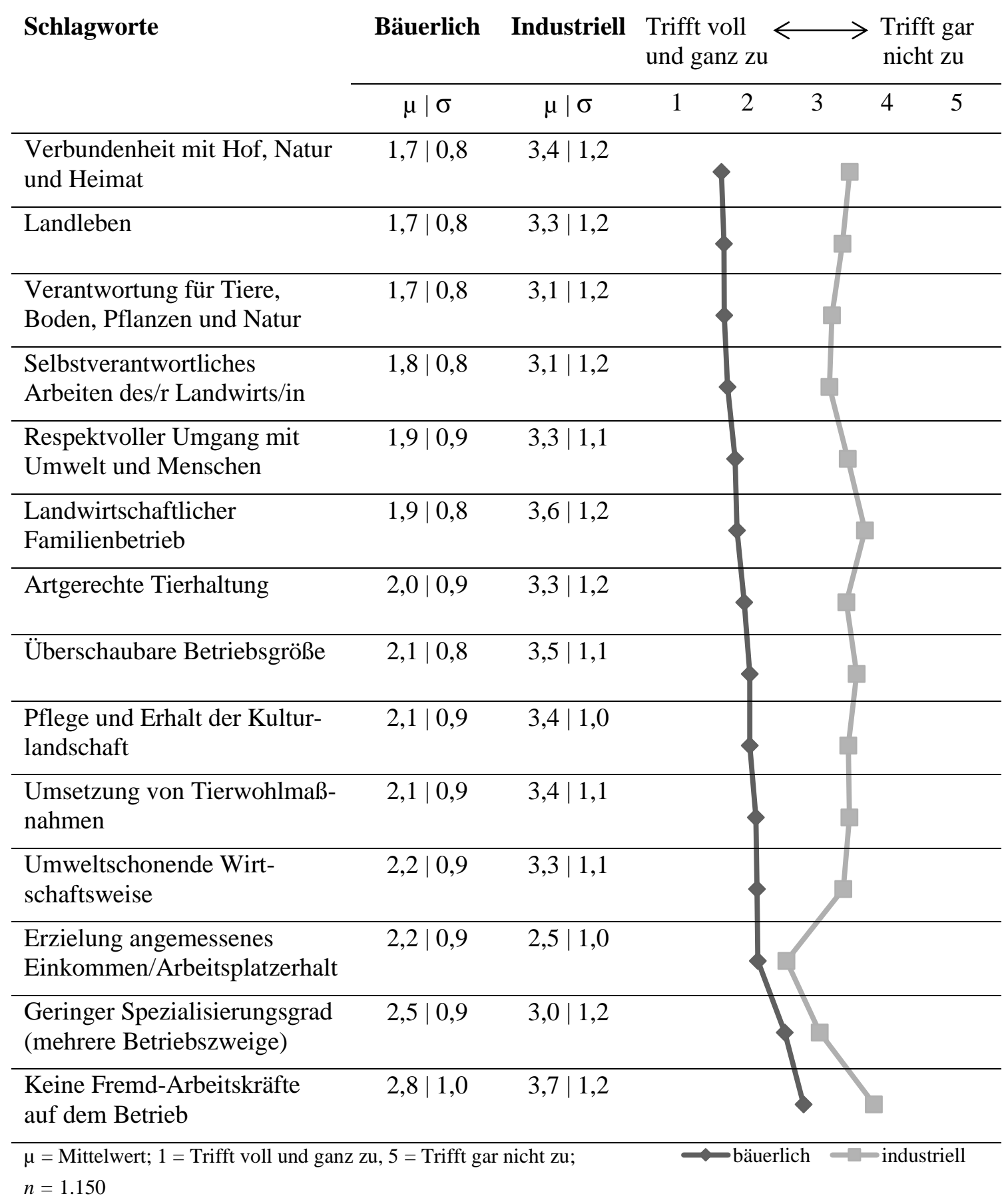

\section{Abbildung 1: Assoziationen mit eher „bäuerlicher“ Landwirtschaft}

\section{Quelle: Eigene Berechnung}

Die Standardabweichungen, als Streuungsmaß der, auf die gestellten Fragen gegebenen Antworten, liegen bei den mit den der „bäuerlichen” Landwirtschaft assoziierten Schlagworten bzw. Kurzaussagen zwischen $\sigma=0,8$ und $\sigma=1,1$. Bezogen auf die ,industrielle” Landwirtschaft, streuen die Werte zwischen $\sigma=0,9$ und $\sigma=1,2$. Die größte Streuung mit $\sigma=1,2$ weisen, bezogen auf den Begriff ,industrielle” Landwirtschaft, die Schlagworte bzw. Kurzaussagen ,,Verantwortung für Tiere, Boden, Pflanzen und Natur”, ,Landleben”, 
„Landwirtschaftlicher Familie und ihre Familie auf dem Betrieb”, „, Verbundenheit mit Hof, Natur und Heimat”, ,, Selbstverantwortliches Arbeiten des/r Landwirts/in” und ,, Geringer Spezialisierungsgrad, sprich mehrere Betriebszweige” auf. Die größte Einigkeit mit $\sigma=0,8$ besteht, verbunden mit dem Begriff der „bäuerlichen” Landwirtschaft, bei den Schlagworten bzw. Kurzausaussagen ,, Verantwortung für Tiere, Boden, Pflanzen und Natur”, „Landleben”, „Landwirtschaftlicher Familienbetrieb”, ,, Verbundenheit mit Hof, Natur und Heimat”, ,,Selbstverantwortliches Arbeiten des/r Landwirts/in” und „Überschaubare Betriebsgröße”.

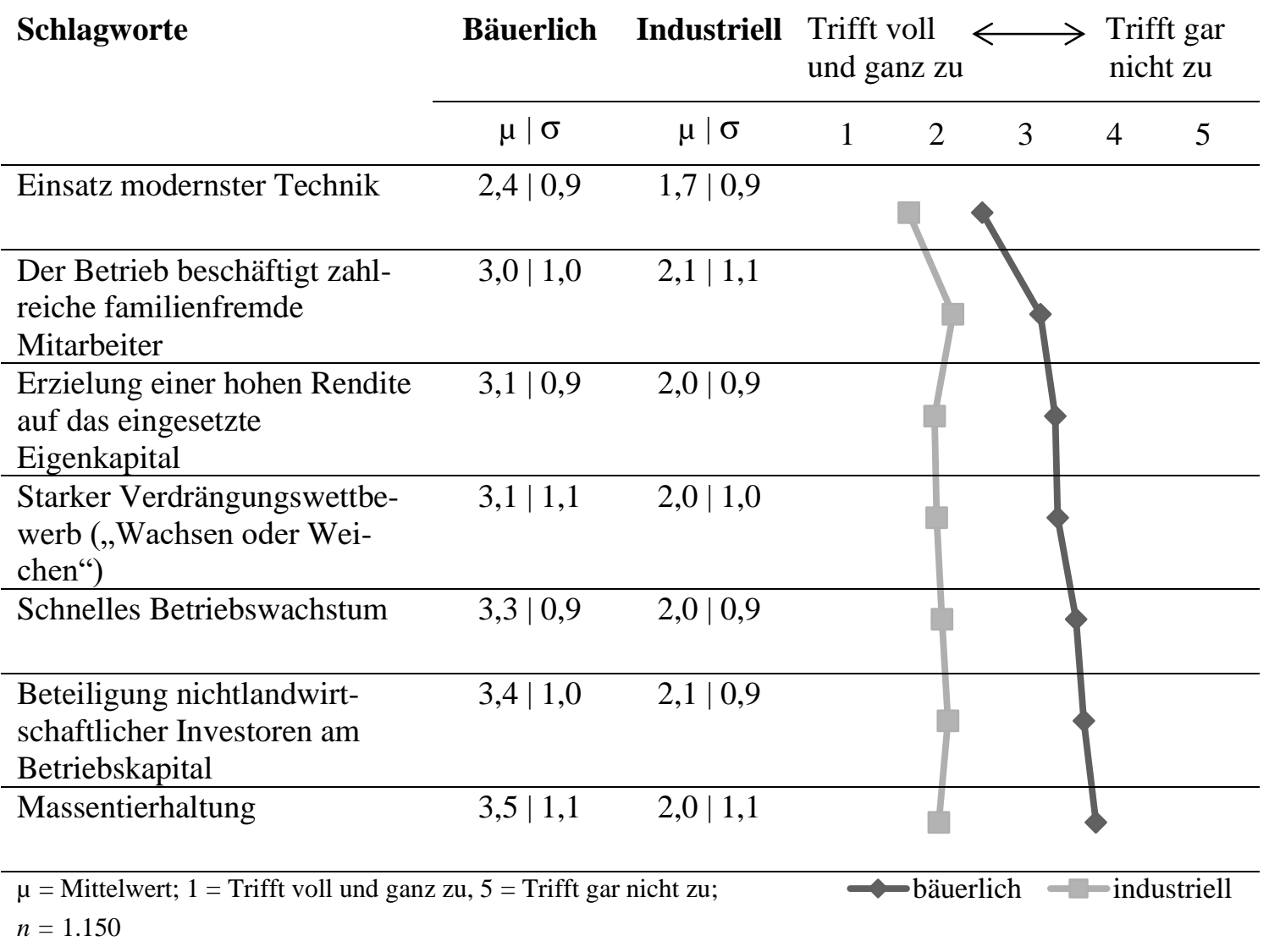

\section{Abbildung 2: Assoziationen mit eher ,industrieller“ Landwirtschaft}

Quelle: Eigene Berechnung

\section{Diskussion und Schlussfolgerungen}

Im Mittelpunkt der Studie stand das Verständnis deutscher Konsumenten von „,bäuerlicher" und ,industrieller“ Landwirtschaft. Bisher existiert bezüglich dieser beiden Begrifflichkeiten keine einheitliche Definition. In dieser Studie wurden verschiedene Merkmale genannt, die von den befragten Konsumenten hinsichtlich der beiden genannten Begriffe zustimmend oder ablehnend bewertet werden mussten, um auf diese Weise zu einem besseren Verständnis der Begriffe ,,bäuerliche“ und ,,industrielle“ Landwirtschaft beizutragen. 
Durch die Ergebnisse wird bestätigt, dass ein Großteil der Befragten eine „bäuerliche“ Landwirtschaft als eine Art Gegenmodell zu konventionellen, von weiten Teilen der Gesellschaft als ,,industrialisiert“ verstandenen Landwirtschaftsbetrieben sieht. Neben diesem deutlich ersichtlichen Kontrast der erfragten Begrifflichkeiten, wurde die ,industrielle“ Landwirtschaft, verglichen mit der „bäuerlichen“, eher mit negativ belegten Begriffen verbunden. Gleichzeitig bezeugen die über die gegebenen Antworten streuenden Standardabweichungen, dass, bezogen auf den Begriff der „,industriellen” und verglichen mit der „,bäuerlichen" Landwirtschaft, eine leicht höhere Uneinigkeit in der Zuordnung der vorgegebenen Schlagworte bzw. Kurzaussagen besteht. Die Streuung der Standardabweichungen vergegenwärtigt außerdem, dass man keineswegs von einem einheitlichen Verbraucherverständnis sprechen kann, was die Ergebnisse anderer Studien unterstreicht (SONNTAG, 2017; ZANDER et al., 2013). Dies gilt es in weitergehenden Arbeiten tiefer zu ergründen. Denkbar wäre beispielsweise die Durchführung einer Clusteranalyse, um die vorliegende Stichprobe in Gruppen mit ähnlichem Antwortverhalten zu unterteilen.

Bezieht man weitere Studien mit ein, scheint sich zu bestätigen, dass ein zum Teil von der Realität abweichendes Bild der Landwirtschaft vorherrscht (ZANDER et al., 2013; WENINGER, 2014). Während in Deutschland bzw. Europa als Folge der damit zusammenhängenden gesellschaftlichen Unzufriedenheit der „bäuerliche“, kleiner strukturierte Familienbetrieb als Gegenmodell zur „,industrialisierten“ Landwirtschaft in den Vordergrund der gesellschaftlichen und politischen Aufmerksamkeit gerät, liegt der gesellschaftliche Fokus in Kanada und den USA eher auf einer gesünderen aber nicht zwingend kleinteiligeren landwirtschaftlichen Produktion (DEVERRE und LAMINE, 2010). Es stellt sich die Frage, worauf diese Diskrepanz fußt. Hier besteht ebenfalls weiterer Forschungsbedarf, um Lösungsansätze zur Klärung dieser verzerrten Wahrnehmung zu finden und so der Gesellschaft ein realitätsnäheres Fundament zur Bewertung landwirtschaftlicher Bewirtschaftungsweisen zu bieten. So wäre es vorstellbar, Experten aus den Bereichen der Landwirtschaft aber auch aus vor- und nachgelagerten Bereichen sowie aus Politik und Verwaltung zu diesem Thema zu befragen und die gewonnen Erkenntnisse mit denen dieser Studie zu vergleichen.

Denkbare Lösungsansätze zur Vermittlung eines realitätsnäheren Bildes der Landwirtschaft, die zum Teil in der wissenschaftlichen Literatur bereits aufgegriffen wurden, bestehen zum einen in mehr Transparenz landwirtschaftlicher Produktionsweisen, um so der Gesellschaft ein realistischeres Bild gegenwärtig praktizierter Landbewirtschaftung zu vermitteln. Ein Beispiel für ein solches, mehr Transparenz schaffendes Vorgehen, stellt die am 01. Januar 2018 in Kraft getretene Stoffstrombilanzverordnung dar. Erklärtes Ziel des Bundesministeriums für Ernährung und Landwirtschaft ist es, mit dieser Verordnung, als 
Teil des sogenannten Düngepakets, Nährstoffflüsse in landwirtschaftlichen Betrieben transparenter zu gestalten (BMJV, 2018). Hier gilt es zu bedenken, dass es in der Regel für alle Seiten eines Konflikts vorteilhafter ist, solchen Problemstellungen zu begegnen, bevor die Politik sich berufen fühlt, ordnungspolitische Maßnahmen zu ergreifen. Zum anderen wird versucht, sich mehr an den Erwartungen der Verbraucher zu orientieren (ZANDER et al., 2013). Als Beispiel eines solchen Ansatzes darf die Initiative Tierwohl gesehen werden. In diesem Branchenbündnis aus Landwirtschaft, Fleischwirtschaft und Lebensmitteleinzelhandel, das in dieser Form einzigartig ist, verpflichten sich die Teilnehmer pro verkauftem Kilogramm Fleisch, einen gewissen Teil den Tierhalter zukommen zu lassen, damit diese vermehrt Tierwohlmaßnahmen, wie beispielsweise mehr Platz in den Ställen, umsetzen können (GESELLSCHAFT ZUR FÖRDERUNG DES TIERWOHLS IN DER NUTZTIERHALTUNG, 2018). Neben den zahlreichen Befürwortern dieser Initiative, gab es aber auch kritische Stimmen unterschiedlicher Interessensvertretungen (HEISE et al., 2017). Darüber hinaus wäre der Einbezug weiterer Stakeholder, wie namenhafte, sowohl die Landwirte aber auch die Verbraucherabsichten vertretende Verbände, denkbar, um auf diese Weise ein gegenseitiges Verständnis zu fördern. Schließlich wäre es für künftige Novellierungen der Gemeinsamen Agrarpolitik (GAP) in Europa für eine bessere Kommunikation wichtig, ein bzgl. der hier untersuchten Begrifflichkeiten einheitliches Vokabular zu verwenden.

\section{Literatur}

ABL (Arbeitsgemeinschaft bäuerliche Landwirtschaft) (2015): Bäuerliche Landwirtschaft ist unsere Zukunftslandwirtschaft. URL: http://www.abl-ev.de/fileadmin/Dokumente/AbL_ev/Agrarpolitik/15-03-Beilage_Bauernstimme-kl.pdf (Abrufdatum: 21.10.2017).

AGRARBÜNDNIS (2001): Leitbild bäuerliche Landwirtschaft. URL: http://www.kasseler-institut.org/fileadmin/kasins/Pospap_AB_B_uerlich.pdf (Abrufdatum: 21.10.2017).

Balmann, A., Chatalova, L., Gagalyuk, T. und V. VAlENTinov (2016): Gesellschaftliche Verantwortung in der landwirtschaftlichen Tretmühle: Moderne Landwirtschaft, technologische Tretmühle und gesellschaftliche Entfremdung - Folgen, Herausforderungen und Lösungsansätze. In: DLG E.V. (Hrsg.): Moderne Landwirtschaft zwischen Anspruch und Wirklichkeit - Eine kritische Analyse (Band 110). DLG-Verlag, München: 147- 170.

BIRNER, R. (2012): Globale Entwicklungen der gesellschaftlichen Akzeptanz moderner Landwirtschaft. In: Landinfo 3 (2012): 19-27.

BMEL (Bundesministerium für Ernährung und Landwirtschaft) (2018): Landwirtschaft verstehen - Fakten und Hintergründe. URL: https://www.bmel.de/SharedDocs/Bilder/Cover/Landwirtschaft-Verstehen.jpg;jsessionid=18ED6C75626F610A4D1982527EEC4917.1_cid296?__blob=poster\&v=6 (Abrufdatum: 23.05.2019).

BMJV (Bundesministerium der Justiz und für Verbraucherschutz) (2018): Verordnung über den Umgang mit Nährstoffen im Betrieb und betriebliche Stoffstrombilanzen. URL: https://www.gesetze-im-internet.de/stoffbilv/index.html (Abrufdatum: 24.10.2018).

BÖHME, K. (2011): Die Kappung im Vorschlag für die Direktzahlungen-Verordnung. In: Briefe zum Agrarrecht 11 (2011): 434-437. 
BÖHME, K. (2014): „Bäuerlicher Familienbetrieb“ - zur agrarrechtlichen Relevanz eines vielgebrauchten Begriffes. In: Briefe zum Agrarrecht 10 (2013): 405-413.

BREUSTEDT, G. (2013): Umverteilung zwischen deutschen und Bundesländern durch größenabhängige Direktzahlungen. URL: https://www.betriebslehre.agric-econ.uni-kiel.de/de/quicklinks-startseite/Umverteilung\%20durch\%20groessensbhaengige\%20Direktzahlungen.pdf (Abrufdatum: 30.10.2017).

BULLER, H. und C. MORRIS (2004): Growing goods: the market, the state, and sustainable food production. In: Environment and Planning A 36 (6): 1065-1108, doi: 10.1068/a35282.

BUSCH, G., KAYSER, M. und A. SPILLER (2013): Factory farming from a consumer's perspective: associations and attitudes. In: Jahrbuch der Österreichischen Gesellschaft für Agrarökonomie 22 (1): 61-70.

DESTATIS (Statistisches Bundesamt) (2016): Zahlen \& Fakten. URL: https://www.destatis.de/DE/ZahlenFakten/Zahlen Fakten.html (Abrufdatum: 20.10.2017).

CAMPBELL, H. (2009): Breaking new ground in food regime theory: corporate environmentalism, ecological feedbacks and the 'food from somewhere' regime. In: Agriculture and Human Values 26 (4): 309- 319, doi: 10.1007/ s10460-009-9215-8.

DEverRe, C. und C. LAMINE (2010): Les systèmes agroalimentaires alternatifs. Une revue de travaux anglophones en sciences sociales. In: Economie Rurale 317: 57- 73, doi: 10.4000/economierurale.2676.

EDELMANN, M. (2013): What is a peasant? What are peasantries? A briefing paper on issues of definition. URL: http://www.ohchr.org/Documents/HRBodies/HRCouncil/WGPleasants/Edelman.pdf (Abrufdatum: 27.05.2019).

EKD (Evangelische Kirche Deutschlands) (2017): Neuorientierung für eine nachhaltige Landwirtschaft. URL: https://www.ekd.de/neuorientierung_landwirtschaft3.html (Abrufdatum: 30.10.2017).

GERKE, J. (2017): Bäuerliche Landwirtschaft. URL: http://ostdeutsche-bodenpolitik.de/2015/01/30/baeuerliche-landwirtschaft-zum-begriff-und-zu-ihrer-zukunft/ (Abrufdatum: 23.10.2017).

GESELLSCHAFT ZUR FÖRDERUNG DES TIERWOHLS IN DER NUTZTIERHALTUNG (2018): Initiative Tierwohl - Einordnung und Ausblick der Initiative Tierwohl 2018. URL: https://initiative-tierwohl.de/wp-content/uploads/2018/05/20180503-ITW-Rechenschaftsbericht.pdf (Abrufdatum: 24.10.2018).

Heise, H., Overbeck, C. und L. Theuvsen (2017): Die Initiative Tierwohl aus Sicht verschiedener Stakeholder: Bewertungen, Verbesserungsmöglichkeiten und zukünftige Entwicklungen. In: Berichte über Landwirtschaft 95 (1): 1-35, doi: 10.12767/buel.v95i1.137.g317.

ISERMEYER, F. (2014): Künftige Anforderungen an die Landwirtschaft - Schlussfolgerungen für die Agrarpolitik. In: Thünen Working Paper 30. Johann Heinrich von Thünen-Institut, Braunschweig.

JANSEN, K. und S. VELLEMA (2004): Agribusiness and society: Corporate responses to environmentalism, market opportunities and public regulation. Zed Books, London.

KANTAR EMNID (2017): Das Image der deutschen Landwirtschaft. URL: https://media.repromayr.de/79/668279.pdf (Abrufdatum: 01.10.2018).

KAYSER, M., BÖHM, J. und A. SPILLER (2012): Zwischen Markt und Moral - Wie wird die deutsche Land- und Ernährungswissenschaft in der Gesellschaft wahrgenommen? In: Schriften der Gesellschaft für Wirtschafts- und Sozialwissenschaften des Landbaus e.V. 47: 329-341.

Kremen, C., ILES, A. und C. BACON (2012): Diversified Farming Systems: An Agroecological, System-based Alternative to Modern Industrial Agriculture. In: Ecology and Society 17 (4): 44-63, doi: 10.5751/ES05103-170444.

LAMINE, C. (2015): Sustainability and Resilience in Agrifood Systems: Reconnecting Agriculture, Food and the Environment. In: Sociologia Ruralis 55 (1): 41-61, doi: 10.1111/soru.12061.

SONNTAG, W. (2017): Zielkonflikte in der Nutztierhaltung - eine empirische Analyse gesellschaftlicher Erwartungen. Dissertation an der Georg-August-Universität Göttingen. 
SPILLER, A., KAYSER, M. und J. BÖHM (2012): Unternehmerische Landwirtschaft zwischen Marktanforderungen und gesellschaftlichen Erwartungen in Deutschland ... aus Sicht der Forschung. In: Schriften der Gesellschaft für Wirtschafts- und Sozialwissenschaften des Landbaus e.V. 47: 1122.

WBA (Wissenschaftlicher Beirat Agrarpolitik BMEL) (2015): Wege zu einer gesellschaftlich akzeptierten Nutztierhaltung. Gutachten, Berlin. URL: http://www. bmel.de/SharedDocs/Downloads/Ministerium/Beiraete/Agrarpolitik/GutachtenNutztierhaltung.pdf?_ blob=publicationFile (Abrufdatum: 23.05.2019).

WENINGER, L. (2014): Das Image der österreichischen Landwirtschaft bei Jugendlichen der 10. und 11. Schulstufe aus bäuerlichem bzw. städtischem Umfeld. Diplomarbeit am Institut für Marketing und Innovation an der Universität für Bodenkultur Wien.

Woodhouse, P. (2010): Beyond Industrial Agriculture? Some Questions about Farm Size, Productivity and Sustainability. In: Journal of Agrarian Change 10 (3): 437-453, doi: 10.1111/j.14710366.2010.00278.x.

ZANDER, K., Isermeyer, F., BÜrgelt, D., Christoph-SChulz, I. B., SAlamon, P. und D. Weible (2013): Erwartungen der Gesellschaft an die Landwirtschaft. Stiftung Westfälische Landwirtschaft, Münster. 


\section{III.2 Der Markt für Bioenergie}

Friedrich Rübcke von Veltheim, Maximilian Deutsch, Lara Drittler, Christian Schaper und Verena Otter

Geleisteter Eigenanteil an dem Beitrag

\begin{tabular}{|c|c|c|c|c|c|}
\hline $\begin{array}{l}\text { Literatur- } \\
\text { recherche }\end{array}$ & $\begin{array}{l}\text { Entwicklung } \\
\text { des } \\
\text { Forschungs- } \\
\text { rahmens }\end{array}$ & $\begin{array}{l}\text { Konzept und } \\
\text { Design der Stu- } \\
\text { die }\end{array}$ & $\begin{array}{l}\text { Auswahl, Aus- } \\
\text { führung und } \\
\text { Entwicklung } \\
\text { von Methoden }\end{array}$ & $\begin{array}{l}\text { Interpretation } \\
\text { der } \\
\text { Ergebnisse }\end{array}$ & $\begin{array}{l}\text { Konzept und } \\
\text { Schreiben des } \\
\text { Artikels }\end{array}$ \\
\hline $30 \%$ & $30 \%$ & $30 \%$ & $30 \%$ & $30 \%$ & $30 \%$ \\
\hline
\end{tabular}

Dieser Beitrag ist so oder in ähnlicher Fassung veröffentlicht in der wissenschaftlichen Zeitschrift „Austrian Journal of Agricultural Economics and Rural Studies “ 28 (22). 


\section{Inhalt}

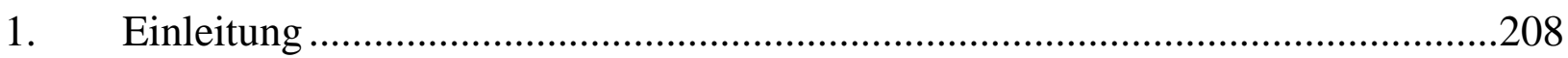

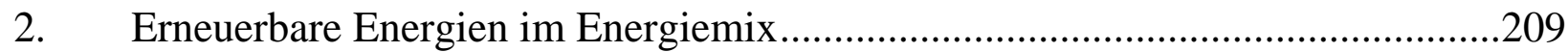

3. Entwicklung der Biomasseerzeugung in Deutschland..........................................

3.1 Biomasse aus landwirtschaftlicher Produktion ...............................................214

3.2 Biomasse aus biogenen Reststoffen und Abfällen ..........................................216

3.3 Biomasse aus forstwirtschaftlicher Produktion...............................................217

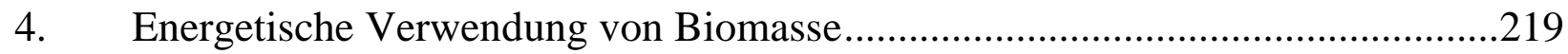

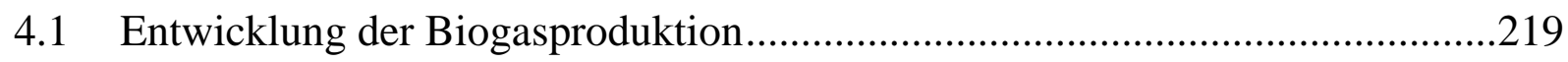

4.2 Entwicklung der Biokraftstoffproduktion ......................................................220

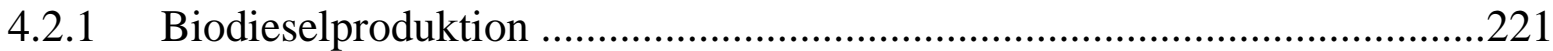

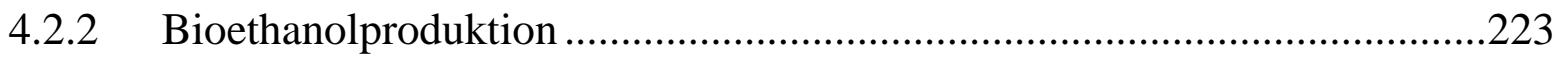

4.3 Strom- und Wärmeerzeugung aus biogenen Festbrennstoffen ...........................227

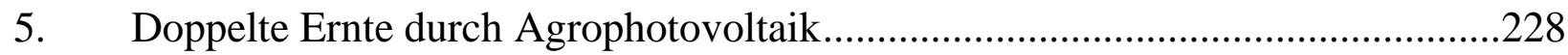

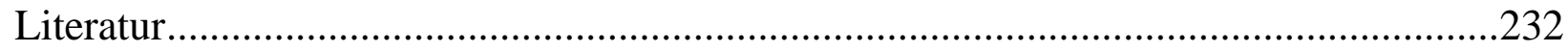




\section{Einleitung}

Die Extremwetterereignisse des vergangenen Jahres, vor allem aber die Dürre in den Sommermonaten 2018, haben die gesellschaftliche und politische Diskussion rund um den Klimawandel und seine Auswirkungen nicht nur innerhalb Deutschlands stark angeheizt (z.B. BMU, 2018a; Die Zeit, 2018; SÜDdeutsche Zeitung, 2018; SPIEGEl OnLine, 2018). Während viele Wirtschaftszweige mit starken Engpässen im Gütertransport zu Wasser fertig werden mussten, hatte die deutsche Landwirtschaft durch die extremen Wetterbedingungen erhebliche, regional stark unterschiedliche Ertragseinbußen zu verzeichnen, sowohl bei Grünland als auch bei vielen Feldfrüchten wie Winterweizen, Wintergerste, Roggen, Mais und Raps (BMEL, 2018). Der volkswirtschaftliche Schaden, der durch die Dürre im Sommer 2018 in Deutschland entstanden ist, wird auf mehrere Milliarden Euro geschätzt (WELT, 2018). Laut einer Studie des Thünen-Instituts aus dem Jahr 2015 ist auch zukünftig mit einem verstärkten Auftreten von extremen Wetterbedingungen zu rechnen. So prognostizieren GöMANN et al. (2015) einen weiteren Anstieg an Hitze- und extrem trockenen Sommertagen, aber auch eine 50 bis $100 \%$ ige Zunahme an Starkregen in den Wintermonaten bis zum Jahr 2100.

Da der Hauptanteil der globalen Treibhausgasemissionen und somit auch das „Global Warming Potential“" auf fossile Brennstoffe zurückzuführen ist, erhalten erneuerbare Energie nun noch stärkere Aufmerksamkeit (IPCC, 2014; KEMFERT, 2018). Denn, Schätzungen zufolge sind die 80 bis 95 \%ige Reduzierung der Treibhausgasemissionen, die zur Abwendung der Auswirkungen des Klimawandels bis 2050 erforderlich wären, nur durch eine konsequente Dekarbonisierung möglich (KEMFERT, 2018). Deshalb beinhalten die Klimaziele der EU und Deutschlands, welche vor dem Hintergrund der Weltklimakonferenz 2015 in Paris, gesteckt worden sind, sowohl eine Verringerung der Treibhausgasemissionen als auch eine Steigerung des Erneuerbare-Energien-Anteils am Bruttoendenergieverbrauch (BMU, 2018b; KEMFERT, 2018). Die Novellierung des Erneuerbare-Energien-Gesetzes (EEG) 2016/2017 sollte zur Erreichung der deutschen Ziele, die kurzfristig wesentlich höher angesetzt sind als die der EU, beitragen, indem es die Förderung durch Ausschreibungsverfahren marktwirtschaftlicher gestaltet (BMWI, 2018a). Dabei minderte es, wie bereits die Novellierungen 2012 und 2014, die relative Vorzugsbehandlung von Biogasanlagen in der Förderung, die während der „Tank oder Teller“-Debatte zunehmend in der Kritik standen, zugunsten von Photovoltaik- und Windanlagen. Auch durch die Konkurrenz zu solchen regenerativen Energien befindet sich die Bioenergiebranche seit einigen Jahren im Schrumpfungsprozess, der ihr in der Energiewende inzwischen nur noch die 
Nebenrolle der Flexibilisierung zuweist (REITER und LINDORFER, 2015; NITSCH, 2017; LANGENBERG et al., 2017). Dies wird auch an den Statistiken des Jahres 2017 deutlich, die, analog zur internationalen Entwicklung, ein besonders großes Investitionswachstum bei den regenerativen Energien Wind und Licht zeigen (BMWI, 2018b; BMWI, 2018c). Auch die Landwirte haben im Jahr 2017 mehr Investitionen in Windkraft- und Photovoltaikanlagen geplant als im Vorjahr. Bei den Photovoltaikanlagen geht der Trend dabei hin zu Dachanlagen, die Solarenergie für den Eigenverbrauch liefern (BAYRISCHES LANDWIRTSCHAFTLICHES WOCHENBLATT, 2017). Freiflächenanlagen waren für die Landwirte von geringerer Attraktivität, da sie bis dato nicht im EEG berücksichtigt und deshalb mit dem Wegfall des Anspruchs auf Agrarsubventionen bei landwirtschaftlichen Flächen verbunden waren. Auch sind Freiflächen-Photovoltaikanlagen mit einem gewissen Flächenverzehr verbunden, der die detaillierte Betrachtung neuer Entwicklungsansätze innovativer, ressourceneffizienter Konzepte zur Doppelnutzung des knappen Faktors Boden, wie z.B. Agrophotovoltaik, dringend erforderlich macht.

\section{Erneuerbare Energien im Energiemix}

Der Primärenergieverbrauch (PEV) gilt als Maß für alle im Inland eingesetzten (Primär-) Energieträger, wie bspw. Braun- und Steinkohle, Mineralöl und Erdgas. Im Jahr 2017 lag der PEV in Deutschland mit etwa 13,6 Exajoule wieder auf dem Niveau von 2011, was einer PEV-Steigerung von etwa $3 \%$ innerhalb der letzten drei Jahre gleichkommt. Auch wenn der Anteil der erneuerbaren Energien 2017 mit $13 \%$ (1.773 Petajoule) über dem von 2011 (11\% - 1.463 Petajoule) lag und sich im Vergleich zum Vorjahr (2016: 1.671 Petajoule) abermals gesteigert hat, erscheint die Erreichung des Ziels der Bundesregierung, den PEV von 2008 bis 2020 um $20 \%$ und bis 2050 sogar um $50 \%$ zu senken, immer weniger wahrscheinlich (STATISTA, 2018a; UMWELTBUNDESAMT, 2018). So war von 2008 bis 2017 lediglich ein Rückgang des PEV um etwa 6 \% zu verzeichnen (UMWELTBUNDESAMT, 2018). Im Zeitraum von 1990 bis 2017 kam es neben der Erhöhung des Anteils erneuerbarer Energien am PEV und einem Rückgang des Braunkohleeinsatzes von etwa $50 \%$, zu einer beträchtlichen Erhöhung des Gasverbrauches (1990: 2.304 Petajoule (15\% des PEV); 2017: 3.242 Petajoule (24 \% des PEV)). Die Mineralöle machen mit etwa $35 \%$ nach wie vor den größten Anteil am PEV aus (UMWELTBUNDESAMT, 2018).

Betrachtet man die jeweiligen Anteile erneuerbarer Energien an der Energiebereitstellung in Deutschland, wird zunächst die hohe Diskrepanz zwischen den einzelnen Bereichen Strom, Wärme (und Kälte) sowie Kraftstoffverbrauch deutlich (Abbildung 1). Den mit Abstand größten Anteil machen die erneuerbaren Energien beim Stromverbrauch aus. 
Verglichen mit 2015 (31,5 \%) und 2016 (31,6 \%), kam es im Jahr 2017 zu einer deutlichen Steigerung der erneuerbaren Energien am Bruttostromverbrauch auf 36,2 \% (STATISTA, 2018d). Somit rangieren die erneuerbaren Energien mit 217 TWh, deutlich vor Braunkohle (2017: 148 TWh) und Kernenergie (2017: 94 TWh), weiterhin auf dem ersten Platz der Bruttostromerzeugung in Deutschland (UMWELTBUNDESAMT; 2018a). Für die Wärmeund Kältebereitstellung kam es im Jahr 2017 gegenüber den Vorjahren mit 13,2 \% zu einem leichten Rückgang des Anteils der erneuerbaren Energien (2016: 13,5 \%; 2015: 13,6 \%). Der Endenergieverbrauch erneuerbarer Energien im Bereich Verkehr bzw. Kraftstoffe blieb in 2017 unverändert auf dem Niveau des Vorjahres, das die Fortsetzung eines leicht rückläufigen Trends darstellte (2017: 5,2\%; 2016: 5,2\%; 2015: 5,3\%) (BMWI, 2018b).



Abbildung 1: Anteile erneuerbarer Energien an der Energiebereitstellung in Deutschland; EEV = Endenergieverbauch

Quelle: Eigene Darstellung nach BMWI (2018)

Insgesamt wurden 2017 etwa 417,8 TWh aus erneuerbaren Energien bereitgestellt, was einer Steigerung von 8 \% zum Vorjahr (2016: 386,4 TWh) entspricht (BMWI, 2018b). Den mit Abstand größten Anteil nahmen dabei die biogenen Brennstoffe für Wärme (37 \%) und Strom (13\%) ein, gefolgt von Windenergie (20\%), Photovoltaik (10\%), Biokraftstoffen (9\%) und Wasserkraft (5\%); Geo- (3\%) und Solarthermie $(2 \%)$ machten hingegen nur einen verhältnismäßig kleinen Teil aus (Abbildung 2). Der aus erneuerbaren Energien 
gewonnene Strom belief sich in 2017 auf etwa 216 TWh, der zum größten Teil aus Windenergie (105,7 TWh), dem Einsatz von Biomasse (50,9 TWh) und Solarenergie (38,0 TWh) gewonnen wurde. Die Bereitstellung von Wärme bzw. Kälte (168,8 TWh) wurde hingegen maßgeblich von der Biomassenutzung $(87,3 \%)$ bestimmt. Der Verbrauch erneuerbarer Energien im Verkehrssektor stieg im Vergleich zum Vorjahr (2016: 33,5 TWh) auf insgesamt 34,5 TWh (2017) an; Biodiesel (61,6 \%) stellte dabei den größten Anteil innerhalb des Sektors bereit (BMWI, 2018b).

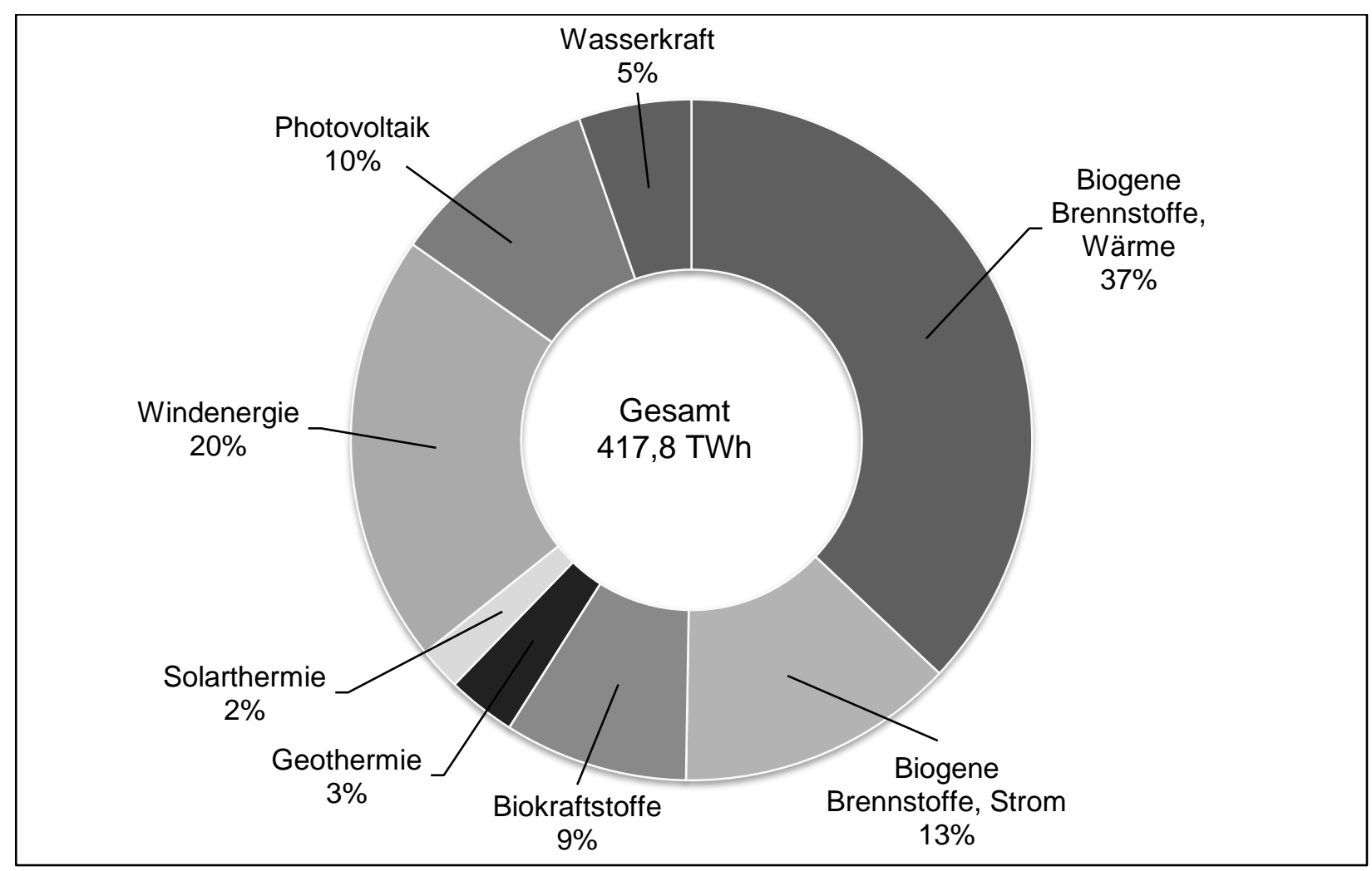

Abbildung 2: Zusammensetzung der erneuerbaren Energien in Deutschland 2017

\section{Quelle: Eigene Darstellung nach BMWI (2018a)}

Durch den fortschreitenden Ausbau des Anteils erneuerbarer Energien am Endenergieverbrauch in Deutschland, konnten im Jahr 2017 etwa 177,1 Mio. t $\mathrm{CO}_{2}$ eingespart werden (+12\% zum Vorjahr). Die Treibhausgasemissionseinsparungen gingen dabei zu $36 \%$ auf Energiegewinnung aus Biomasse, zu $40 \%$ auf Windenergie und zu $15 \%$ auf Solarenergie zurück (BMWI, 2018c). Dabei wurden 76,3\% der $\mathrm{CO}_{2}$-Einsparungen durch Stromerzeugung, 19,5\% durch Wärmeproduktion und 4,2 \% im Verkehrsbereich verwirklicht (BMWI, 2018b).

Erneuerbare-Energien-Anlagen (EEA) haben sich in Deutschland zu einem bedeutenden Wirtschaftsfaktor entwickelt. Dabei stieg die Summe der Investitionen in die Errichtung von EEA innerhalb der vergangenen 18 Jahre stark an; 2010 erreichte sie mit 27,9 Mrd. $€$ ihren bislang höchsten Wert. Nach 2010 schmolz die Investitionssumme kontinuierlich, 
bis sie 2015 einen Wert von 13,8 Mrd. € erreichte. Von 2015 bis 2017 erhöhte sich das Gesamtinvestitionsvolumen in die Errichtung von EEA auf 15,5 Mrd. € (2017), was eine Steigerung von 2,3 \% zum Vorjahr darstellt (BMWI, 2018b). Auch im Jahr 2017 entfielen die mit Abstand größten Investitionen innerhalb der Errichtung von EEA auf die Windenergie (10,7 Mrd. €); 2016 waren es 10,3 Mrd. € (ibid.). In die Errichtung von Wasserkraftanlagen wurde mit einem Umfang von etwa 0,02 Mrd. $€$ hingegen am wenigsten investiert.

Im Jahr 2016 kam es erstmals seit 2011 zu einem Anstieg der Bruttobeschäftigung durch erneuerbare Energien in Deutschland. Sie wuchs von 328.600 Personen im Jahr 2015, auf 338.600 Personen (+3\%) im Jahr 2016, wovon die Windenergie mit 160.100 Personen $(47,3 \%)$ weiterhin die beschäftigungsstärkste Technologiebranche war (BMWI, 2018c, BMWI, 2018d). Ihr folgen der Biomasse- (105.600 Personen; 31,2 \%), der Solarenergie(45.300 Personen; 13,4\%), der Geothermie- (20.300 Personen; $6 \%$ ) und der Wasserkraftbereich (7.400 Personen; 2,2\%) (BMWI, 2018d).

Ein ähnlicher Trend lässt sich auch in der globalen Betrachtung des Einsatzes und Ausbaues erneuerbarer Energien ausmachen. So werden in der Wind- und Solarenergie international die größten Wachstumsraten verzeichnet und in diesen Technologiesparten auch die größten Potenziale zur Deckung des weltweit wachsenden Energiebedarfs gesehen (BMWI, 2018d; REN21, 2018). Daneben wohnt vielen der eingesetzten Erneuerbare-Energien-Technologien durch ihren dezentral einsetzbaren Charakter der Vorteil inne, auch in abgelegenen, ruralen Gegenden eine häusliche oder dörfliche Energieversorgung gewährleisten zu können (BMWI, 2018d). REN21 (2018) geht davon aus, dass im Jahr 2016 etwa $18,2 \%$ des gesamten Endenergieverbrauchs aus erneuerbaren Energiequellen stammt, wobei lediglich 10,4\% auf moderne und 7,8 \% auf traditionelle bzw. veraltete Nutzungsformen zurückgehen. Gerade letztere sind aus ökologischer Sicht in der Regel kritisch zu beurteilen, da sich diese meist auf die Wärmebereitstellung aus Holzkohle und Brennholz beziehen und oft nicht nachhaltig gewonnen werden. Der globale Primärenergieverbrauch ist in 2017 auf den historischen Höchstwert von 13.511,2 Millionen Tonnen Öläquivalent angestiegen, was einer Steigerung von 1,9\% zum Vorjahr entspricht (STATISTA, 2018e). Den Löwenanteil davon machen die Energieträger Erdöl (34,2 \%), Kohle (27,5 \%) und Erdgas $(23,4 \%)$ aus. Erneuerbare Energien kommen lediglich auf einen Anteil von rd. $3,6 \%$.

Bei der globalen Stromerzeugung kam es ebenfalls zu einer zunehmenden Mitbestimmung von erneuerbaren Energien. Im Jahr 2017 nahm ihr Anteil am gesamten Stromverbrauch, verglichen mit dem Vorjahr (2016: 24.765 TWh) um rd. $3 \%$ zu und erreichte einen Wert 
von 25.518 TWh (26,5 des Gesamtstromverbrauchs) (REN21, 2018). Maßgebend bei der Stromerzeugung aus erneuerbaren Energien ist noch immer die Wasserkraft mit einem Anteil von 4.184 TWh (16,4 \%). Das im Jahr 2017 verzeichnete Wachstum geht vor allem auf den Ausbau von Windenergie (2016: 991 TWh; 2017: 1.430 TWh) und Photovoltaik (2016: 371 TWh; 2017: 494 TWh) zurück (BMWI, 2018d; REN21, 2018). Die gesamte installierte Stromerzeugungsleistung belief sich am Ende des Jahres 2017 auf rd. 2.195 Gigawatt (GW). Es wurden global $178 \mathrm{GW}$ zugebaut, was $17 \mathrm{GW}$ mehr sind als im Jahr 2016 (ibid.). Die Hauptrollen spielten hier Photovoltaik- (+98 GW; $402 \mathrm{GW}$ gesamt) und Windkraftanlagen (+52 GW; $539 \mathrm{GW}$ gesamt). Doch trotz des verstärkten Zubaus von Photovoltaik- und Windkraftanlagen, machte im Jahr 2017 noch immer die Wasserkraft den größten Anteil (1.114 GW) der global installierten Stromerzeugungsleistung aus. Dabei konnte China seine globale Führungsrolle im Bereich der erneuerbaren Energien weiter ausbauen. So gingen von den $52 \mathrm{GW}$ Windenergiezubau allein 19,7 GW und von den 98 GW Photovoltaikzubau 53 GW auf China zurück (BMWI, 2018d). Bezogen auf die weltweit installierte Leistung aus EEA, rangiert China (2017: $619 \mathrm{GW}$ ) mit deutlichem Abstand auf dem ersten Platz, vor den USA (2017: $230 \mathrm{GW})$, Brasilien (2017: $128 \mathrm{GW}$ ), Deutschland (2017: 113 GW) und Indien (2017: 106 GW) (STATISTA, 2018f).

Im Vergleich zum Vorjahr folgte dem deutlichen Rückgang der globalen Investitionen in die Stromerzeugung aus erneuerbaren Energiequellen ein Anstieg von 2,1\% (2016: 274 Mrd. \$; 2017: 279,8 Mrd. \$) (BMWI, 2018d). Ausschlaggebend dafür waren allen voran die Entwicklungen in der Photovoltaikbranche (FRANKFURT SCHOOL-UNEP CENTRE/BNEF, 2018). Dabei entfiel der größte Anteil (127 Mrd. \$) auf China, die ihren Photovoltaikanlagenausbau massiv vorantreiben. Dagegen kam es in Europa zu einem Rückgang der Investitionen auf rd. 41 Mrd. \$ (-36 \%), wofür vor allem ein Investitionseinbruch in Großbritannien verantwortlich gemacht werden kann (BMWI, 2018d). Mexiko, Australien und Schweden konnten im Jahr 2017 auf die stärksten Investitionssteigerungen zurückblicken. Die meisten Investitionen flossen 2017 weltweit in den Ausbau der Photovoltaiktechnik (161 Mrd. \$; +12\% zum Vorjahr). Die Windenergie musste dagegen aufgrund gesunkener Technologiekosten einen Rückgang der Investitionen auf 107 Mrd. \$ ($12 \%$ zum Vorjahr) verzeichnen (BMWI, 2018d). Die weltweite Beschäftigtenzahl im Bereich der erneuerbaren Energien stieg 2017 auf 10,3 Mio. Beschäftigten (+5,3 \% zum Vorjahr) weiter an; davon 3,4 Mio. Beschäftigte in der Photovoltaik- und 2 Mio. Beschäftigte in der Biokraftstoffbranche (IRENA, 2018). 


\section{Entwicklung der Biomasseerzeugung in Deutschland}

\subsection{Biomasse aus landwirtschaftlicher Produktion}

Die landwirtschaftlich genutzte Fläche nimmt mit 50,9 \% der Gesamtfläche von 35,7 Mio. ha den größten Teil der Flächennutzung Deutschlands ein (DESTATIS, 2018). Von dieser landwirtschaftlichen Nutzfläche wurden 2017 Schätzungen zufolge 2,65 Mio. ha $(14,55 \%)$ zum Anbau von nachwachsenden Rohstoffen genutzt (FNR, 2018a). Nach einem rasanten Wachstum zu Beginn des Jahrzehnts zeigte sich zuletzt eine Stagnation auf dem Niveau der vorherigen 3 Jahre, mit einem geschätzten leichten Rückgang in 2017 (Abbildung 3).

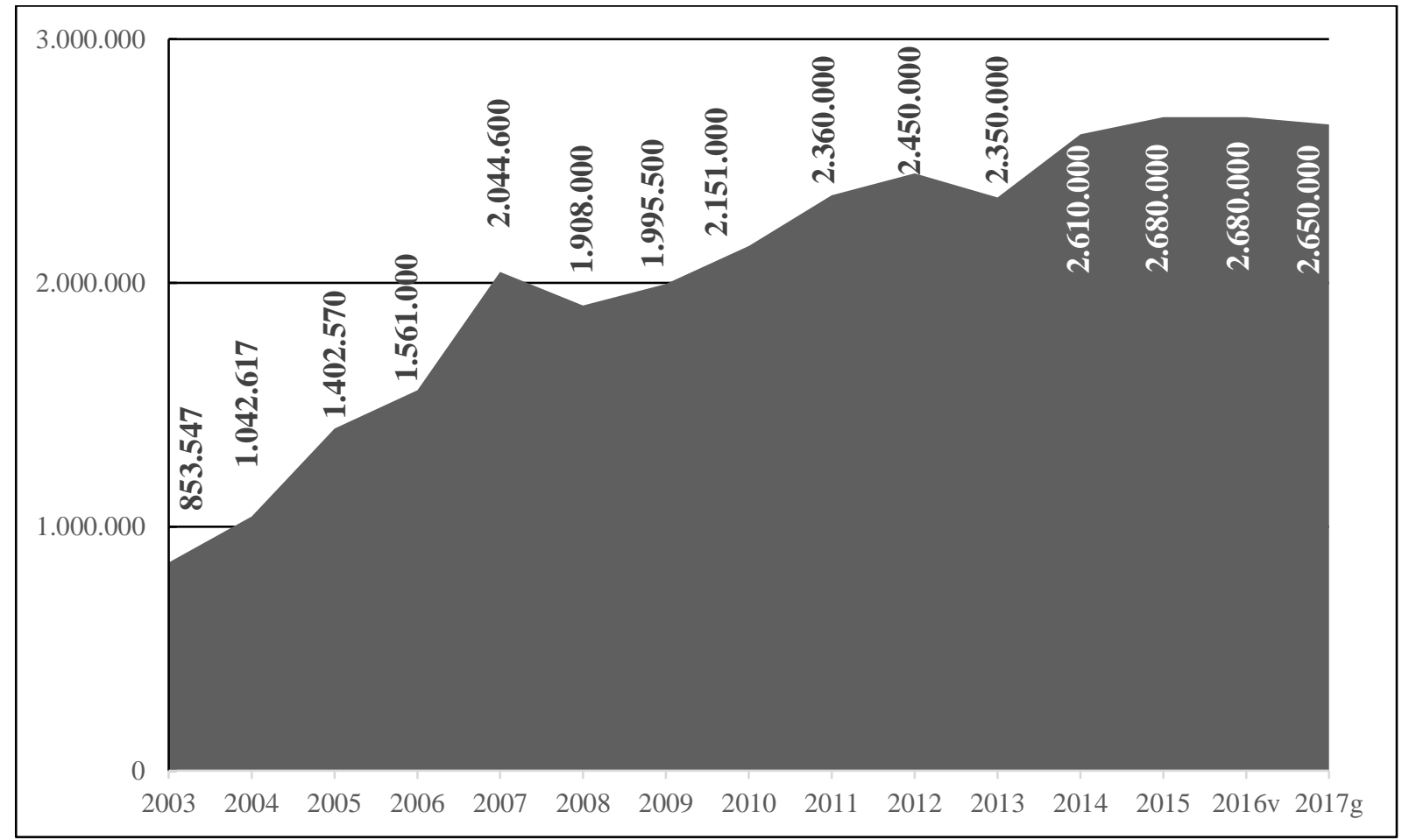

Abbildung 3: Anbaufläche für nachwachsende Rohstoffe in Deutschland (ha); Anbaufläche für 2016 vorläufig (v) und 2017 geschätzt (g)

Quelle: Eigene Darstellung nach FNR (2018a)

Den flächenmäßig größten Anteil an der NawaRo-Anbaufläche haben dabei mit $89 \%$ (2,35 Mio. ha) im Jahr 2017 die Energiepflanzen (Tabelle 1). Hiervon entfallen, trotz eines leichten Rückgangs im Vergleich zum vorherigen Jahr, auf die Pflanzen für Biogasproduktion etwa 1.374.000 Hektar (52\% der NawaRo-Fläche), wobei Mais analog zu den Vorjahren mit 913.000 Hektar den größten Anteil stellt und im Vergleich zu 2016 in der Anbaufläche zugenommen hat. Bezogen auf die gesamte Maisanbaufläche in Deutschland entspricht dies einem Anteil von $36 \%$ für Biogas-Mais und damit einem Rückgang um $2 \%$ im Vorjahresvergleich (FNR, 2018b). Die zweitwichtigste Energiepflanze war, mit einer erneut 
leicht zurückgegangenen Anbaufläche von 713.000 Hektar, Raps für die Biodiesel- und Pflanzenölproduktion. Dies entspricht einem Anteil von $27 \%$ an der gesamten NawaRoFläche. Analog zu den Vorjahren hält hier der Effekt der Energiesteuererhöhung 2013 für Biodiesel und Pflanzenöl (BMJV, 2018a) an, wobei 2017 auf weiterhin niedrigem Niveau eine leichte Steigerung von Produktion und Absatz stattgefunden hat (FNR, 2018c). Inwiefern dieser Trend jedoch, insbesondere vor dem Hintergrund des rückläufigen Absatzes von Dieselfahrzeugen im Zuge der sogenannten „Dieselkrise“ (STATISTA, 2018g), langfristig anhält, bleibt abzuwarten. Energiepflanzen für die Bioethanolherstellung hatten 2017 einen prozentual gestiegenen Anteil von gut 9,5\% (251.000 ha) an der gesamten NawaRo-Fläche. Auch hier haben die niedrigen Rohstoffpreise einen Einfluss auf Produktion und Absatz gehabt, darüber hinaus ist parallel zum genannten Rückgang der Dieselfahrzeuge eine Steigerung der Zulassungen von Fahrzeugen mit Benzinmotor festzustellen (STATISTA, 2018h). Mittel- bis langfristig wird hierbei auch der zu erwartende, steigende Anteil von Elektrofahrzeugen ein bedeutender Einflussfaktor für die Anbaufläche von Biokraftstoffgrundstoffen sein. Der Anbau sonstiger Energiepflanzen wie Agrarholz und Miscanthus ist analog zum Vorjahr mit 11.000 ha auf sehr niedrigem Niveau stagnierend. Industriepflanzen zur stofflichen Nutzung konnten 2017 im Vergleich zu den Energiepflanzen ihre Anbaufläche erneut steigern und machen jetzt $11 \%$ der gesamten NawaRo-Fläche aus (300.000 ha). Die dominierenden Kulturen bleiben dabei Raps zur Herstellung von technischem Rapsöl (131.000 ha) sowie stärkehaltige Pflanzen zur Industriestärkeproduktion (128.000 ha), wobei das Flächenwachstum im Vergleich zum Vorjahr primär auf den gestiegenen Anteil des Zuckerrübenanbaus für Industriezucker zurückzuführen ist. 
Tabelle 1: Anbau von Energie- und Industriepflanzen in Deutschland (ha)

\begin{tabular}{|c|c|c|c|c|c|c|c|}
\hline & Rohstoff & 2013 & 2014 & 2015 & $2016^{*}$ & $2017 * *$ & $\begin{array}{c}\text { Anteil } \\
\text { an Na- } \\
\text { waRo- } \\
\text { Fläche } \\
2017^{* *} \\
(\%)\end{array}$ \\
\hline \multirow{5}{*}{ 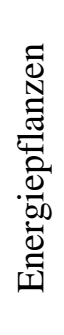 } & Raps für Biodiesel/Pflanzenöl & 614.000 & 799.000 & 805.000 & 720.000 & 713.000 & 26,91 \\
\hline & Zucker/Stärke für Bioethanol & 173.000 & 188.000 & 238.000 & 259.000 & 251.000 & 9,47 \\
\hline & Pflanzen für Biogas & 1.269 .000 & 1.354 .000 & 1.340 .000 & 1.394 .000 & 1.374 .000 & 51,85 \\
\hline & $\begin{array}{l}\text { Sonstiges (u.a. Agrarholz, } \\
\text { Miscanthus) }\end{array}$ & 9.000 & 10.500 & 11.000 & 11.000 & 11.000 & 0,42 \\
\hline & Energiepflanzen insgesamt & 2.060 .000 & 2.350 .000 & 2.390 .000 & 2.380 .000 & 2.350 .000 & 88,68 \\
\hline \multirow{8}{*}{ 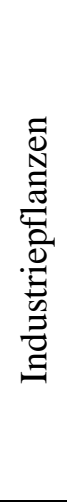 } & Industriestärke & 107.000 & 106.000 & 108.000 & 128.000 & 128.000 & 4,83 \\
\hline & Industriezucker & 17.000 & 12.500 & 12.300 & 12.800 & 15.400 & 0,58 \\
\hline & Technisches Rapsöl & 139.000 & 116.000 & 138.000 & 132.000 & 131.000 & 4,94 \\
\hline & Technisches Sonnenblumenöl & 7.500 & 6.000 & 7.100 & 7.740 & 7.740 & 0,29 \\
\hline & Technisches Leinöl & 3.500 & 3.500 & 3.500 & 3.500 & 3.500 & 0,13 \\
\hline & Pflanzenfaser & 500 & 1.000 & 1.490 & 1.520 & 1.520 & 0,06 \\
\hline & Arznei- und Farbstoffe & 12.000 & 12.000 & 12.000 & 12.000 & 12.000 & 0,45 \\
\hline & Industriepflanzen insgesamt & 286.000 & 257.000 & 283.000 & 298.000 & 300.000 & 11,32 \\
\hline \multicolumn{2}{|c|}{ NawaRo insgesamt } & 2.350 .000 & 2.610 .000 & 2.680 .000 & 2.680 .000 & 2.650 .000 & 100,00 \\
\hline
\end{tabular}

*vorläufige Werte, **geschätzte Werte; Abweichungen in den Summen ergeben sich durch Rundungen Quelle: Eigene Darstellung nach FNR (2018a)

\subsection{Biomasse aus biogenen Reststoffen und Abfällen}

Weiterhin von großer Bedeutung für die Bioenergie bleiben die biogenen Rest- und Abfallstoffe, dies sind organische Stoffe, die als Nebenprodukt bei der nicht-energetischen Nutzung von Biomasse entstehen (FNR, 2015; MüHLENHOFF und DANNEMANN, 2017). Hierzu zählen insbesondere Grünschnitt, Landschaftspflegematerial, Exkremente aus der Tierhaltung, Stroh, Waldrestholz sowie organische Abfälle aus dem privaten (z.B. Biomüll) und dem gewerblichen Bereich (z.B. Schlachtabfälle), wobei den mit Abstand größten Anteil die forstwirtschaftlichen Reststoffe und landwirtschaftliche Nebenprodukte haben (73\%). Das technische Gesamtpotenzial biogener Rest- und Abfallstoffe beträgt jährlich 98,4 Mio. $t$ Trockensubstanz, von denen derzeit bereits $69 \%$ genutzt werden (BOHNET et al., 2017; FNR, 2015). Das größte ungenutzte technische Biomassepotenzial in Bezug auf die Energiemenge liegt in der Nutzung von Waldrestholz (49\% des gesamten ungenutzten Potenzials) und Getreidestroh (31\%). Die nicht genutzten Potenziale im festen oder flüssigen Mist von Rindern und Schweinen liegen dagegen bei jeweils nur $3 \%$ bis $5 \%$ des gesamten ungenutzten Potenzials (FNR, 2015). Die Verwertung dieser biogenen 
Rest- und Abfallstoffe ist jedoch in doppelter Hinsicht von besonderer Bedeutung: Zum einen erhöht sie die Akzeptanz von Bioenergieanlagen durch die Verringerung des nötigen Energiepflanzenanbaus (ZSCHACHE et al., 2010; KRÖGER et al., 2016). Zum anderen stellt ihre Nutzung einen der wenigen Bereiche für den wirtschaftlichen Neubau von Biogasanlagen dar, wenn auch auf geringem Niveau (DOTZAUER et al., 2018).

Der Anfall von biogenen Rest- und Abfallstoffen bleibt regional sehr unterschiedlich. Während insbesondere Regionen mit intensivem Ackerbau und hohen Bodenqualitäten das größte Potenzial an Stroh aufweisen, fallen die meisten tierischen Exkremente in Regionen hoher Nutztierdichte wie Nordwestdeutschland an (MÜHLENHOFF und DANNEMANN, 2017). Aufgrund ihres relativ niedrigen Energiegehalts machen diese energiebezogen nur $14 \%$ des gesamten Biomasseeintrags aus; ihr Substrateinsatz liegt hingegen bei rund $44 \%$ der gesamten Masse (MÜHLENHOFF, 2013; KRÖGER et al., 2016). Um die Transportwürdigkeit der Exkremente zu steigern und ihren Einsatz auch außerhalb der Regionen hoher Nutztierdichte zu erhöhen, findet vermehrt eine Separation zur Auftrennung in flüssige und feste Bestandteile statt (KRÖGER et al., 2016). Aufgrund der durch die Einführung der Düngeverordnung (BMJV, 2018b) verringerten Ausbringungszeiten und -mengen könnte sich hier ein vermehrter Trend zur bioenergetischen Nutzung tierischer Exkremente ergeben. Als zukünftige Entwicklungsbereiche werden die Flexibilisierung der Energiebereitstellung aller Anlagen, hochflexible Anlagen- und Rohstoffnutzungskonzepte (z.B. verbesserte Nutzbarmachung des Strohanteils) sowie Kostenreduzierung und Effizienzsteigerung gesehen (DOTZAUER et al., 2018).

Der Anfall von Waldrestholz findet primär in den waldreichen Regionen Süddeutschlands statt, wobei in 2018 durch Borkenkäferkalamitäten und Trockenstress auch in den nordund östlichen Bundesländern mit einer erhöhten Einschlags- und damit Restholzmenge zu rechnen ist. Diese Bioenergiepotenziale haben eine hohe Bedeutung für die zukünftige Bioenergienutzung, da sie auch in Regionen mit hoher Einwohnerzahl anfallen und daneben in den meisten Regionen Deutschlands den gesamten Haushaltsstrombedarf allein decken könnten (BOHNET et al., 2017).

\subsection{Biomasse aus forstwirtschaftlicher Produktion}

Die Landfläche der Bundesrepublik Deutschland ist zu $32 \%$ bewaldet (ca. 11,4 Mio. ha); damit ist Deutschland eines der waldreichsten Länder Europas. Auf $56 \%$ der Waldfläche wachsen Nadelbäume, auf $44 \%$ Laubbäume, wobei der Nadelbaumanteil seit einigen Jahren abnimmt und der Mischwaldanteil zunimmt (BMEL, 2017; SDW, 2018a). Nahezu die Hälfte des Waldes befindet sich im Privatbesitz (48 \%); 57 \% der Betriebe sind kleiner als 
20 ha, nur $13 \%$ der Betriebe sind größer als 1.000 ha. Rund 1,3 Mio. ha Wald befinden sich im landwirtschaftlichen Besitz. Den Ländern gehören $29 \%$ des Waldes und der Anteil des Körperschaftswaldes beträgt $19 \%$. Die restlichen $4 \%$ befinden sich im Besitz des Bundes; dabei handelt es sich v.a. um Waldflächen entlang von Straßen sowie Waldflächen, die militärisch genutzt werden (SDW, 2018c). Die Verteilung der Waldflächen innerhalb Deutschlands ist recht heterogen. Die norddeutsche Ebene wird insbesondere durch die Landwirtschaft gekennzeichnet - der Waldanteil ist dementsprechend gering (Schleswig-Holstein: $11 \%$ ). Das Mittelgebirge ist hingegen besonders waldreich (Hessen: $42 \%$ ) (BMEL, 2017). Die im letzten Jahrzehnt in Deutschland zu beobachtende Zunahme der Waldfläche um 0,4 \% (SDW, 2018a) steht im Kontrast zu der Abnahme der Waldfläche weltweit (BOLTE et al., 2016): Einem Waldverlust von 58.000 ha stehen 108.000 ha Zugewinn an Wald gegenüber (BMEL, 2017). Diese positive Entwicklung in Deutschland ist den rechtlichen Rahmenbedingungen geschuldet. Mittels Eingriffs-Ausgleichs-Regelung des Bundesnaturschutzgesetzes wird die Aufforstung landwirtschaftlicher Nutzflächen gefördert und eine Rodung von Waldflächen mit anschließender Umwandlung der Nutzungsart ist gemäß des Bundeswaldgesetztes nur in Ausnahmefällen zulässig (BMJV, 2018c; BMJV, 2018d).

Der jährliche Holzzuwachs liegt bei durchschnittlich 11,2 $\mathrm{m}^{3} / \mathrm{ha}$ bzw. insgesamt bei 121,6 Mio. $\mathrm{m}^{3}$. Der jährliche Holzeinschlag beträgt durchschnittlich 7,0 $\mathrm{m}^{3} / \mathrm{ha}$ bzw. insgesamt 76 Mio. $\mathrm{m}^{3}$. Aufgrund des vermehrten Holzzuwachses ist der Holzvorrat auf insgesamt ca. $336 \mathrm{~m}^{3} / \mathrm{ha} \mathrm{bzw}$. 3,7 Mrd. $\mathrm{m}^{3}$ angewachsen (BMEL, 2017). Damit befindet sich der Holzvorrat auf einem Rekordniveau (SDW, 2018b). Der jährliche Holzverbrauch in Deutschland liegt bei 132 Mio. $\mathrm{m}^{3}$, wobei der Anteil des Waldrohholzes $58 \%$ beträgt. Rund zwei Drittel des eingeschlagenen Rohholzes werden stofflich genutzt; Verwendungsbereiche sind der Wohnungsbau und die Produktion von Holzwerk- und Zellstoffen sowie Papier. Einer direkten energetischen Nutzung wird rund ein Drittel des eingeschlagenen Rohholzes zugeführt (BMEL, 2017). Holz ist somit nicht nur ein wichtiger Industrierohstoff, sondern auch der bedeutendste Bioenergieträger (BUNZEL et al., 2011; KALTSCHMITT et al., 2010). Insgesamt werden jährlich rund 64 Mio. $\mathrm{m}^{3}$ Holz energetisch genutzt; der Anteil des Waldrestholzes an der energetischen Nutzung liegt bei 42,2\%, die restlichen $57,8 \%$ stammen insbesondere aus Reststoffen der stofflichen Holzverwertung (BMEL, 2017). 


\section{Energetische Verwendung von Biomasse}

\subsection{Entwicklung der Biogasproduktion}

Durch die Überbauung von Biogasanlagen zur flexibleren Stromerzeugung wurden im Jahr 2017 die Stromerzeugungskapazitäten aus Biogas um 315 MW erhöht, was, verglichen mit dem Vorjahr, einer 55\%igen Steigerung (2016: +202 MW) des Zubaus entspricht (BMWI, 2018d). Für 2018 wird von einem Rückgang des Zubaus um 36 MW ausgegangen und die installierte elektrische Leistung von Biogasanlagen mit 4.843 MW (inkl. Einspeisung durch Biomethan) vorhergesagt (FvB, 2018). Doch gerade aufgrund des Zugewinns an Kapazität durch eine Überbauung der Anlagen im Rahmen der Flexibilisierung, ist der Einfluss auf den Umfang des tatsächlich aus Biogas erzeugten Stroms im Jahr 2017 relativ gering. Hier kam es lediglich zu einem Anstieg von 0,2 \% (2016: 29,26 Mrd. KWh; 2017: 29,32 Mrd. KWh) (BMWI, 2018d). Damit rangiert die Stromgewinnung aus Biogas, wie auch im Vorjahr, auf dem dritten Platz der Stromerzeugung durch erneuerbare Energien, hinter der Windenergie (48,8\%) und Photovoltaik (18,2 \%). Der Trend eines wenn auch verhaltenen Anstiegs der Anzahl neu gebauter Biogasanlagen konnte in 2017 fortgeführt werden (Abbildung 4). Für das Jahr 2018 wurde der Bau von 163 (2017: 122) neuen Anlagen prognostiziert, was einem Anstieg der Gesamtanzahl an Biogasanlagen auf 9.494 (+1,7\% im Vergleich zum Vorjahr) entspricht (FvB, 2018). Der vergleichsweise verhaltene Anstieg der Zahl der Biogasanlagen in den letzten Jahren ist vor allem auf die diversen Novellierungen des Erneuerbare-Energien-Gesetzes (EEG) zurückführen; zu nennen ist unter anderem der Wegfall substrat- oder technikbezogener Prämien nach 2014. Die Anzahl der Beschäftigten im Biogassektor lag im Jahr 2017 bei 47.000. Für das Jahr 2018 wird keine Veränderung der Anzahl der Arbeitsplätze prognostiziert (FVB, 2018). 


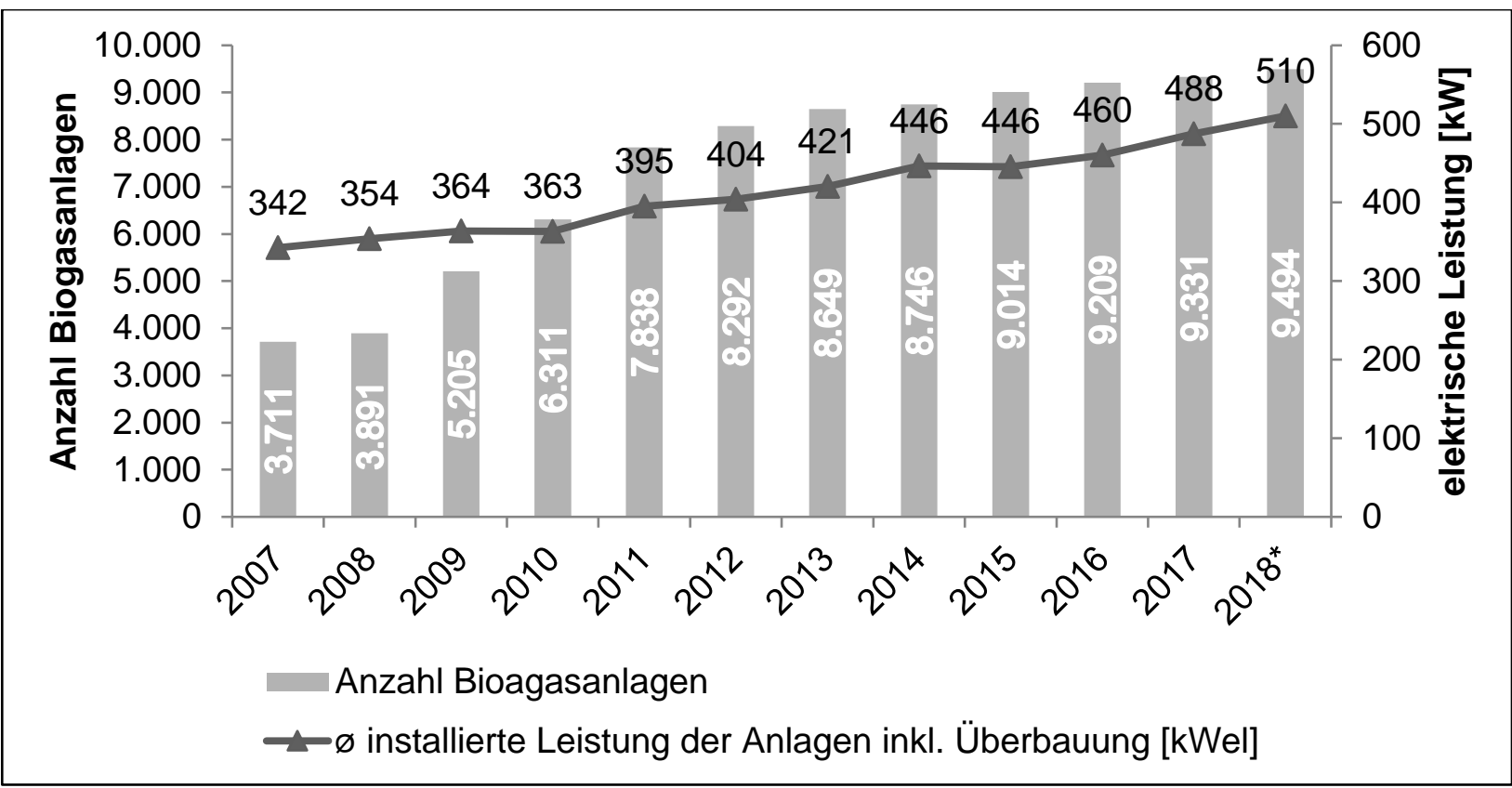

Abbildung 4: Entwicklung der Zahl der Biogasanlagen und der durchschnittlichen installierten Leistung; *Prognose

Quelle: Eigene Darstellung nach STATISTA (2018b; 2018c)

\subsection{Entwicklung der Biokraftstoffproduktion}

Der Klimawandel wird als eine der größten Herausforderungen dieses Jahrhunderts eingeschätzt. Um die Auswirkungen möglichst gering zu halten, hat die EU die Dekarbonisierung als ein politisches Ziel gefestigt (EUROPEAN COMMISSION, 2018; DRITTLER et. al, 2018). Vor diesem Hintergrund trat im Mai 2018 die neue Verordnung (2018/842/EG) in Kraft, die die Festlegung verbindlicher nationaler Jahressziele für die Reduzierung der THG-Emissionen im Zeitraum 2021 bis 2030 regelt. Für Deutschland gilt nunmehr ein Minderungsziel von $38 \%$ gegenüber dem Referenzjahr 2005. In Zahlen bedeutet dies für die hiesige Land- und Forstwirtschaft eine THG-Minderungsvorgabe von ca. 14 Mio. t CO 2 -̈̈quiv. bis 2030 (Ufop, 2018). Um die gesetzten Ziele zu erreichen, stehen verschiedene Maßnahmen zur Flexibilisierung zur Verfügung. Eine der zentralen Herausforderungen, um die gesteckten Ziele zu erreichen, stellt die in 2050 auf mehr als neun Milliarden Menschen gewachsene Weltbevölkerung und das damit einhergehend steigende globale Verkehrsaufkommen dar, das insbesondere in den Schwellenländern zunehmen wird. Dabei stellt sich die Frage nach der Gestaltung des Transformationsprozesses, also dem Umstieg auf effiziente und bezahlbare THG-neutrale alternative Kraftstoffe und Antriebe (UfOP, 2018; VDB, 2019). In diesem Zusammenhang stehen Politik und Wirtschaft unter einem massivem Handlungs- bzw. Innovationsdruck. Von Seiten der Biokraftstoffbranche werden dabei verschiedene Bereiche von entscheidender Bedeutung sein und 
den Markt zukünftig in die richtige Richtung zu beeinflussen. Auch nachhaltige Biokraftstoffe müssen dabei kurz- bis mittelfristig ihren Beitrag leisten. Biodiesel und Bioethanol haben historisch gesehen die höchste „Integrationsfähigkeit“ in bestehende Verarbeitungsund Distributionsstrukturen nachgewiesen. Eine Elektrifizierung über sehr große Strecken ist in vielen Flächenländern beispielsweise nicht machbar. Der Verbrennungsmotor wird daher seine Perspektive behalten, bei gleichzeitiger Verbesserung der Verbrauchseffizienz und der Abgasqualität. In diesem Umfeld wird der Kraftstoffmix aufgrund unterschiedlicher Produktionsverfahren und Rohstoffherkünfte vielfältiger werden (UFOP, 2018). Von entscheidender Bedeutung ist es dabei, dass sich die Ziele in den nationalen Energie- und Klimaplänen widerspiegeln müssen, die die EU-Mitgliedsstaaten der EU-Kommission bis 2019 und die Unterzeichnerstaaten des Pariser Klimaschutzabkommens bis 2020 vorlegen müssen. Die wichtigste Entscheidung für die zukünftige Marktentwicklung nach 2020 ist somit in der zukünftigen EU-Politik zu sehen (PUTTKAMMER und GRETHE, 2015).

In Deutschland belief sich der Kraftstoffverbrauch im Jahr 2017 auf insgesamt 57,6 Mio. t Kraftstoffe (2016: 56,7 Mio. t; 2015: 56 Mio. t; 2014: 55 Mio. t). Dabei entfielen in 2017 63,8 \% (2016: 63,4 \%) auf Diesel- und 30,5 \% (2016:30,1 \%) auf Ottokraftstoffe. Der Anteil biogener Kraftstoffe lag, wie auch in 2016, bei 4,7 \% (bezogen auf den Energiegehalt). Der Wert für den Anteil biogener Kraftstoffe am Gesamtkraftstoffverbrauch mit rd. 3,4 Mio.t (2016:3,35 Mio. t) stagniert damit weitestgehend auf den Vorjahreswerten. Biodiesel ist in Deutschland mit rd. 2,1 Mio. t und einem Marktanteil von 60,29\% nach wie vor der wichtigste Biokraftstoff, gefolgt von Bioethanol mit rd. 1,6 Mio.t (33,97\%), hydrierten Pflanzenölen mit 163.200 t (4,79 \%), Biomethan mit 28.000 t (0,82 \%) und Pflanzenölen mit $4.000 \mathrm{t}(0,12 \%)$ (FNR, 2018f).

\subsubsection{Biodieselproduktion}

In 2017 stagnierte die Biodieselproduktion in Deutschland mit 3,1 Mio. $t$ auf dem Vorjahresniveau. 2015 lag dieser Wert bei 3,01 Mio. $t$ und in 2014 bei 3,0 Mio. $t$ (Abbildung 5) (FNR, 2018f). Auch die Produktionskapazitäten verblieben auf dem Vorjahresniveau von 3,89 Mio. t; die Kapazitätsauslastung der Biodieselanlagen betrug in 2017 aber nach wie vor rd. $80 \%$. Der Biodieselabsatz im Inland blieb annähernd konstant und erhöhte sich leicht von rd. 1,98 Mio. $t$ in 2016 auf 2,05 Mio. $t$ in 2017 (FNR, 2018f). Abbildung 5 zeigt auch, dass durch die veränderten politischen Rahmenbedingungen Biodiesel seit 2008 deutliche Absatzeinbußen verzeichnet. Auch durch die Einführung der THG-Quote seit dem 01.01.2015 stagniert der Absatz von Biodiesel weiter (DRITTLER et al., 2018) 


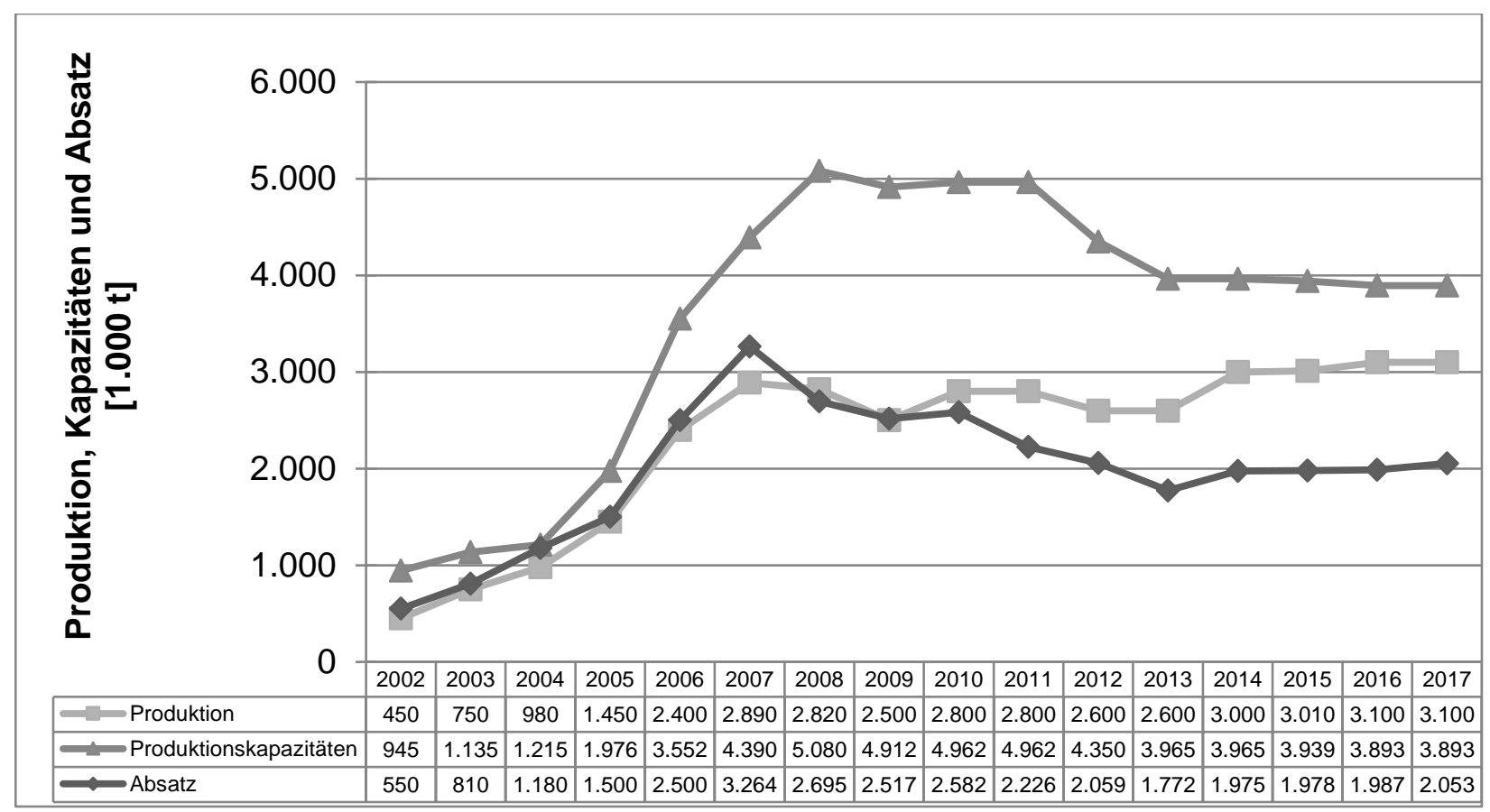

Abbildung 5: Biodieselkapazitäten, -produktion und -absatz in Deutschland

Quelle: Eigene Darstellung nach FNR (2018f)

Die EU-27 ist nach wie vor der weltweit größte Hersteller von Biodiesel. Die Erzeugung belief sich in 2017 einschließlich der Produktion von hydrierten Pflanzenölen (HVO) auf rd. 10,39 Mio. t (2016: 10,61 Mio. t). Somit stagnierte in 2017 die Produktion, der Absatz und der Verbrauch im Vergleich zu 2016 weiter. Gegenüber 2016 (20,3 Mio. t) haben sich die Biodieselproduktionskapazitäten in der EU etwas erhöht und liegen aktuell bei rd. 21,12 Mio. t. Dieser Wachstumsschritt vollzieht sich aber weitestgehend auf den Bereich von hydriertem Pflanzenöl (HVO), der sich bisher auf die Niederlande, Finnland, Italien, Frankreich und Spanien konzentriert. Schwerpunktländer der europäischen Biodieselproduktion sind nach wie vor Deutschland (2017: 3,1 Mio. t; 2016: 3,2 Mio. t), Frankreich (2017: 1,7 Mio. t; 2016: 1,8 Mio. t), Spanien (2017: 1,5 Mio. t; 2016: 1,1 Mio. t), Polen (2017: 0,9 Mio. t; 2016: 0,80 Mio. t) und die Niederlande (2017: 0,5 Mio. t; 2016: 0,64 Mio. t) (UfOP, 2018). In der EU wird bis 2020 mit keinen signifikanten Änderungen bei der Biodieselherstellung gerechnet (BWK, 2018).

Weltweit stieg der Biodiesel- einschließlich des HVO-Verbrauchs im Jahr 2016 von 28,57 Mio. $t$ auf 28,84 Mio. $t$ in 2017 an. Neben der EU mit einem Verbrauch von 10,83 Mio. $t$ sind vor allem die USA mit 6,45 Mio. $t$ sowie Brasilien 3,37 Mio. $t$ und Argentinien mit 1,17 Mio.t zu nennen. Die Betrachtung der weltweiten Produktionsmengen von Biodiesel zeigt ein ähnliches Bild. Mit einer Produktionsmenge von 10,4 Mio. t Biodiesel liegt die EU-27 nach wie vor an der Spitze, gefolgt von den USA mit rd. 6 Mio. t, Brasilien mit 4,3 Mio.t, Argentinien mit 3,3 Mio.t und Indonesien mit 2,5 Mio.t 
(Abbildung 6) (STATISTA, 2019; UFOP, 2018). Experten gehen für die nächsten Jahre u.a. wegen der Konkurrenz zum Nahrungs- und Futtermittelmarkt, der Ausweitung der Ölanbauflächen insbesondere in Südostasien sowie des begrenzten Klimagasminderungspotenzials von nur eingeschränkten Zuwächsen aus. Allerdings kann der Biodieselmarkt zum Teil von den Entwicklungen auf dem Futtermittelmarkt profitieren. Insgesamt ist bis 2020 nur von geringen Mengenzuwächsen bei der globalen Biodieselproduktion auszugehen. Innerhalb des Biodieselsortimentes ist mit einer wachsenden Bedeutung von HVO-Kraftstoffen zu rechnen. Neben den europäischen Produzenten stellen vor allem die USA und Singapur wachsende HVO-Kraftstoffmärkte dar (BWK, 2018).

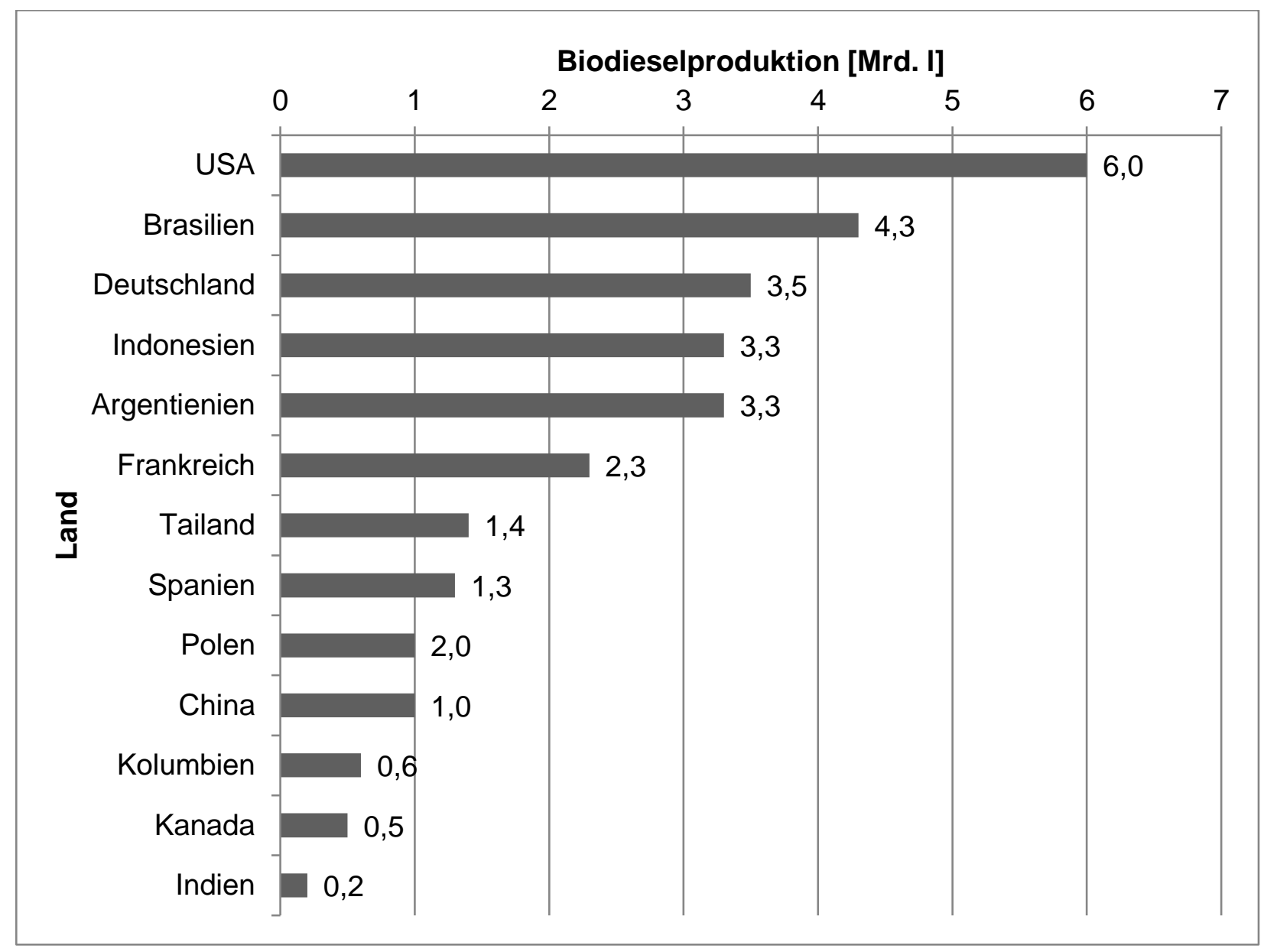

Abbildung 6: Weltweit größte Biodieselproduzenten (2017)

Quelle: Eigene Darstellung nach STATISTA (2019)

\subsubsection{Bioethanolproduktion}

Laut dem Bundesverband der deutschen Bioethanolwirtschaft (BDBE) stieg die Produktion von Bioethanol in Deutschland bis zum Jahr 2015 kontinuierlich auf insgesamt 739.821,0 t an. Für das Jahr 2016 konnte ein leichter Rückgang der Produktion auf 738.169,0 t festgestellt werden. Auch in 2017 verzeichnete die Branche einen weiteren 
Rückgang der Produktion um rd. $9 \%$ auf 673.000,0 t (BDBE, 2018). Die Produktion von Bioethanol aus Industrierüben, Futtergetreide sowie Reststoffen und Abfällen betrug in 2017672.930 t. Dies entspricht einem Rückgang um 8,8 \% gegenüber dem Vorjahr mit einer Produktionsmenge von 738.169 t. Aus Industrierüben wurden 141.401 t Bioethanol und damit $26 \%$ weniger als in 2016 (191.270 t) hergestellt (FNR, 2018f). Der Rückgang der Bioethanolproduktion auf Rübenbasis ist vor allem auf die mehrmonatige Stilllegung eines Bioethanolwerkes zurückzuführen (BDBE, 2018). Weiterhin wurden aus Futtergetreide 522.638 t Bioethanol hergestellt (-2,2 \%; 2016: 534.589 t). Dabei wurden 2,1 Mio. $\mathrm{t}$ Futtergetreide (4,7 \% der deutschen Getreideernte von 45,5 Mio. t im Jahr 2017) als Rohstoff zur Bioethanolproduktion eingesetzt. Aus Reststoffen und Abfällen wurden rd. $9.000 \mathrm{t}$ Bioethanol gewonnen, was einem Minus von $28 \%$ gegenüber dem Vorjahr (12.310 t) entspricht (DRITTLER et al., 2018; BDBE, 2018).

Auf dem deutschen Benzinmarkt wurde in 2017 ein Absatz von rd. 18,3 Mio. t erreicht. Bioethanol erreichte am gesamten Benzinmarkt einen fast unveränderten Anteil von 6,0 Vol.-Prozent (2016: 6,1 Vol.-Prozent). Im Jahr 2017 wurden knapp 1,2 Mio. t Bioethanol für Kraftstoffanwendungen verbraucht. Trotz eines insgesamt höheren Benzinverbrauchs ist ein leichter Rückgang des Bioethanolverbrauchs um 1,6 \% gegenüber dem Vorjahr zu verzeichnen gewesen. Die wichtigste Verwendung von Bioethanol in Deutschland ist die Beimischung zu Benzin für die Sorten Super, Super Plus (E5) und Super E10, gefolgt von der Verwendung als Benzinadditiv ETBE (Ethyl-Tertiär-Butylether). Super E5 erreichte mit 15 Mio. $\mathrm{t}$ in 2017 einen Marktanteil von 82,1\%. Im Vorjahr waren es 15,1 Mio. $t$ mit einem Marktanteil von 82,8 \% gewesen. Der Marktanteil von Super Plus lag mit rd. 830.000 t ähnlich wie im Vorjahr auf 4,5\%. Der Absatz der bis zu $10 \%$ Bioethanol enthaltenden Kraftstoffsorte Super E10 stieg in 2017 auf 2,4 Mio. t. Dies bedeutet einen Marktanteil von 13,4\% und einem leichten Zuwachs gegenüber dem Vorjahr mit 12,6 \%. Der Verbrauch von ETBE sank von $128.761 \mathrm{t}$ auf 111.400 t Bioethanol (BDBE, 2018). Nach ersten Schätzungen wird in Deutschland für 2018 eine leicht positive Entwicklung von Produktion und Verbrauch erwartet. Ein Grund wird in der gestiegenen Treibhausgas-Minderungsquote liegen, die sich in diesem Jahr positiv auf den Einsatz von Bioethanol als Beimischung zu Benzin auswirken wird. Erste Prognosen des Bafas weisen darauf hin, dass von Januar bis Ende April 2018 der Verbrauch von Bioethanol im Vergleich zum entsprechenden Vorjahreszeitraum um mehr als $9 \%$ gestiegen ist (BDBE, 2018).

Die Bioethanolproduktion in der EU erreichte im Jahr 2015 rd. 5,3 Mrd. 1 (Abbildung 7). Bis 2015 profitierte die Branche von niedrigen Rohstoffpreisen und restriktiven 
Maßnahmen für Bioethanolimporte. Seit 2016 stiegen Verbrauch und Produktion von Kraftstoffethanol nur noch sehr langsam und lagen bei 5,34 Mrd. 1. In 2017 lag die Bioethanolproduktion bei rd. 5,84 Mrd. 1. Auch für 2018 wird mit 5,84 Mrd. 1 keine weitere Steigerung prognostiziert (EPURE, 2018; USDA, 2017). Frankreich ist mit 970 Mio. 1 weiterhin größter Produzent vor Deutschland mit 950 Mio. 1, Ungarn mit 640 Mio. 1, Belgien mit 560 Mio. 1, die Niederlande mit 450 Mio. 1 und Spanien mit 400 Mio. 1. Die deutsche Bioethanolnachfrage machte 2015 mit 1,17 Mio. t einen europaweiten Anteil von 27,2 \% aus und liegt damit deutlich vor Frankreich (0,67 Mio. t) und dem Vereinigten Königreich (0,63 Mio. t) (BDBE 2018). Das Potenzial von Bioethanol in Bezug auf die schnelle und kostengünstige Senkung von Treibhausgasemissionen wird aktuell nicht weiter ausgeschöpft. Jedoch wird mit der weiteren Umsetzung der ,Erneuerbare-Energien-Richtlinie“, die für das Jahr 2020 im Verkehrssektor $10 \%$ erneuerbare Energien verbindlich vorschreibt, in den Ländern der EU nach wie vor mit einer zunehmenden Nachfrage nach Bioethanol gerechnet. Die Kapazitäten zur Produktion von Bioethanol belaufen sich in der EU zurzeit auf rd. 9,3 Mrd. 1 und sind zu ca. 85 \% (5,84 Mrd. 1) ausgelastet. Die Produktionskapazitäten haben sich seit 2012 nicht signifikant erhöht; auch in 2018 wird nicht mit weiteren Anlagenerweiterungen gerechnet (USDA, 2017; EPURE, 2018).



Abbildung 7: Der Bioethanolmarkt in der EU (2009 bis 2017)

Quelle: Eigene Darstellung nach USDA (2017) 
Die Welt-Ethanolproduktion lag in 2015 bei rd. 97,2 Mrd. 1 (Abbildung 8). Davon entfallen auf die USA 56,0 Mrd. 1, auf Brasilien 26,8 Mrd. 1, auf Europa 5,25 Mrd. 1, auf China 3,0 Mrd. 1 und auf Kanada 1,65 Mrd. 1 (Abbildung 8; RFA, 2019). In 2016 stieg die Weltethanolproduktion nur leicht auf 97,6 Mrd. 1 an. Vor allem weiteten die USA und Brasilien ihre Produktion etwas aus. Die USA produzierten in 2016 rd. 58 Mrd. 1, Brasilien 27,6 Mrd. 1. In der EU nahm die Produktion leicht ab und bewegte sich bei rd. 5,25 Mrd. 1. China baute die Vorjahresproduktion von 3,0 Mrd. 1 auf knapp 3,2 Mrd. 1 weiter aus und hat sich als viertgrößter Ethanolproduzent in der Welt etabliert. In 2017 belief sich die Weltethanolproduktion auf rd. 103 Mrd. 1. Vor allem die USA haben ihre Produktion noch einmal auf knapp über 60 Mrd. 1 steigern können. In Brasilien stagnierte die Menge mit einer Produktion von rd. 26,7 Mrd. 1 genauso wie in Europa mit 5,6 Mrd. 1. China behauptete seine Position als viertgrößter Bioethanolproduzent mit einer Menge von 3,3 Mrd. 1. Ferner sind im asiatischen Raum noch Thailand mit 1,4 Mrd. 1 und Indien mit rd. 1,0 Mrd. 1 als bedeutende Erzeugerländer zu nennen. Experten sehen vor allem in der Ausweitung der brasilianischen Produktion ein zukünftiges Wachstumspotenzial des Marktes, da Brasilien zunehmend die steigende Inlandsnachfrage decken muss. Aber auch dem asiatischen Raum werden weitere Wachstumschancen zugesprochen (OECD-FAO, 2018).

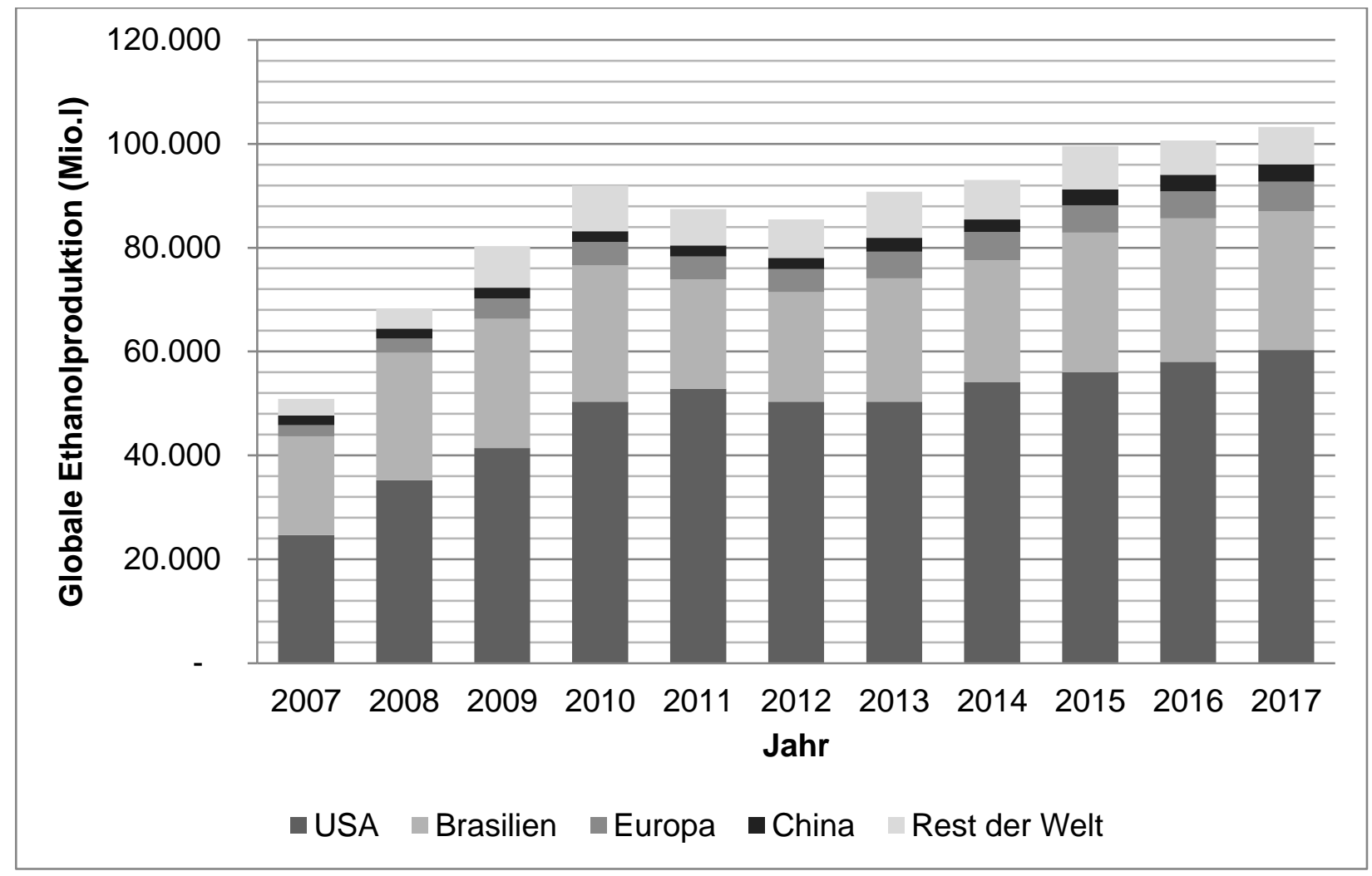

Abbildung 8: Globale Ethanolproduktion (2007 bis 2017)

Quelle: Eigene Darstellung in Anlehnung an RFA (2019) 


\subsection{Strom- und Wärmeerzeugung aus biogenen Festbrennstoffen}

Bei biogenen Festbrennstoffen handelt es sich um Brennstoffe organischer Herkunft wie z.B. Waldrestholz, Holzkohle, Heu oder Rapsstroh; sie liegen zum Zeitpunkt ihrer energetischen Nutzung in fester Form vor. Innerhalb der Biomasse, die zur energetischen Nutzung eingesetzt wird, nehmen biogene Festbrennstoffe den bedeutendsten Anteil ein. Verfügbare und technisch nutzbare biogene Festbrennstoffe werden in Rückstände/Nebenprodukte (z.B. Waldrest- und Schwachholz, Stroh, Industrierestholz, Bau- und Altholz) und speziell angebaute Energiepflanzen (Holz aus Kurzumtriebsplantagen, Miscanthus) unterteilt. Des Weiteren wird zwischen holzartigen, halmartigen und sonstigen Brennstoffen unterschieden (FNR, 2014). Biogene Festbrennstoffe werden ohne vorherige Umwandlung in Feuerungsanlagen zur Strom- und Wärmerzeugung eingesetzt (LFU, 2018).

Elektrische Energie aus biogenen Festbrennstoffen wird mittels einer Turbine erzeugt, die durch Wasserdampf angetrieben wird. Der Wasserdampf entsteht durch thermische Energie, die bei der Verbrennung von biogenen Festbrennstoffen in Biomassekraftwerken bzw. Biomasseheizkraftwerken entsteht. Die im Wege der Kraft-Wärme-Kopplung in Biomasseheizkraftwerken gleichzeitig produzierte Wärme findet darüber hinaus als Fern-, Nah- oder Prozesswärme Verwendung; aufgrund der geringen Abgabe ungenutzter Abwärme an die Umgebung können bei der Kraft-Wärme-Kopplung Wirkungsgrade von bis zu $90 \%$ erreicht werden (SCHAUMANN und SCHMITZ, 2010). Insgesamt wurden in 2017 rund 51,4 TWh Strom aus Biomasse erzeugt, davon 20,7 \% aus biogenen Festbrennstoffen (FNR, 2018d).

Der Anteil erneuerbarer Energien am Endenergieverbrauch für Wärme und Kälte ist in 2017 um 0,3 \% auf 13,2 \% gesunken. Die absolute Nutzung erneuerbarer Wärme ist um 2,6\% von 164,5 TWh in 2016 auf 168,8 TWh in 2017 angestiegen; gleichzeitig ist jedoch auch ein Anstieg fossiler Energieträger im Wärmesektor zu verzeichnen. Biogene Festbrennstoffe nehmen dabei mit ca. $67 \%$ (rund $113 \mathrm{TWh}$ ) den bedeutendsten Anteil an der erneuerbaren Wärmebereitstellung ein (UMWELTBUNDESAMT, 2018b). Holz ist der wichtigste Bioenergieträger in der Wärmeerzeugung aus biogenen Festbrennstoffen. In Deutschland verfügt ca. jeder vierte Haushalt über eine Holzfeuerung zur Wärmebereitstellung. Insgesamt gibt es ca. 16 Mio. Feuerstätten für Holzbrennstoffe, in denen jährlich ca. 38 Mio. Festmeter Holz verfeuert werden. Scheitholz aus dem Wald stellt dabei den bedeutendsten Holzbrennstoff dar. Mit Einzelraumfeuerstätten (z.B. Kaminöfen oder Grund- und Kachelöfen), die eine Ergänzung zur Zentralheizung darstellen und nur gelegentlich genutzt werden, werden v.a. in privaten Haushalten einzelne Wohnbereiche beheizt. Holzzentralheizungen (z.B. Scheitholzvergaserkessel, Pelletheizungen, 
Hackschnitzelheizungen) versorgen hingegen alle Räume eines Gebäudes über eine wasserführende Zentralheizung mit Wärme und erwärmen zugleich das Brauchwasser der Haushalte; in Deutschland verfügen ca. eine Million Haushalte über eine Holzzentralheizung (FNR, 2018e).

\section{Doppelte Ernte durch Agrophotovoltaik}

Für die sich immer wieder entfachende Diskussion der Flächennutzungskonkurrenz, sei es zur Produktion von Lebensmitteln oder zur direkten bzw. indirekten Gewinnung von Energie, existieren seit längerem verschiedene Lösungsansätze. Ein solcher Ansatz zur Begegnung dieses auch als „Tank oder Teller“-Debatte bekannten Disputs ist die komplementäre Nutzung landwirtschaftlicher Flächen durch Agrophotovoltaik (VOGELPOHL, 2018; PHILIPPS et al., 2018). Hierunter versteht man die Kombination von Photovoltaikanlagen mit darunter angebauten Feldfrüchten, um so eine ressourceneffizientere Doppelnutzung des knappen Faktors Boden zu erzielen.

Dazu gibt es verschiedene Umsetzungsmöglichkeiten. Eine davon sieht die Errichtung von Gewächshäusern vor, die beispielsweise gerahmte, kristalline Glas-Glas-Module, die in regelmäßigen Abständen zueinander auf einer etwa 2,20 Meter hohen Unterkonstruktion angeordnet sind, zur Stromgewinnung nutzen. Durch die transparente Folie, in die die Zellen zwischen den Modulgläsern laminiert sind, dringen noch etwa 20 bis $30 \%$ des Sonnenlichts zu den Pflanzen vor (UlRICH, 2018). Die Leistung der Module bleibt dabei auf einem vergleichbaren Niveau, wie die herkömmlicher Standardmodule. Damit eine solche Anlage ökonomisch sinnvoll betrieben werden kann, müssen nach Angaben des Unternehmens Sunfarming wenigstens 60 Hektar landwirtschaftliche Fläche überdacht werden, aus der eine Leistung von bis zu 30 Megawatt gewonnen werden kann (ibid.). Bisher zielen solche Konzepte vor allem auf die Errichtung in sonnenreichen Regionen der Erde ab. Entsprechende Testanlagen stehen bereits in afrikanischen, asiatischen und karibischen Ländern, wo auch netzferne Anlagen z.B. zur Aufbereitung von Salz- zu Süßwasser eingesetzt werden könnten. Gerade die teilweise Beschattung der darunter liegenden Pflanzen kann in Ländern des Sonnengürtels eine positive Auswirkung auf den Pflanzenertrag haben, da diese sonst Gefahr laufen zu „verbrennen“.

Ein anderes, derzeit in Deutschland im Test befindliches Konzept sieht die Installation von Agrarphotovoltaik-Freiflächenanlagen (APV) auf 4 bis 7 Meter hohen Gestellen vor. Auf diese Weise kann die überbaute Fläche weiterhin durch Landmaschinen bewirtschaftet werden. Die Abschattung der Nutzpflanzen kann durch verschieden große Abstände 
zwischen den APV-Reihen angepasst werden, um so bestmöglich den Bedürfnissen der Pflanzenkultur begegnen zu können. Pflanzen, die über eine gewisse Schattentoleranz verfügen, oder sogar vom Halbschatten profitieren, kommen hierfür besonders infrage. Bisher haben sich vor allem Gerste, Kartoffeln, Kohl, Raps, Salat oder Spargel bewährt (TROMMSDORF, 2018). Auf einer aktuellen Testfläche am Bodensee werden auch Weizen, Sellerie und Kleegras hinsichtlich Ertragsentwicklung untersucht. In diesem, durch das BMBF geförderte FONA-Projekt „APV-RESOLA“ werden bifaziale Solarmodule verwendet, die mit etwas größerem Reihenabstand als bei klassischen Freiflächenanlagen in 5 Metern Höhe installiert wurden (ISE, 2018a). Es wurde untersucht, welchen Einfluss die Beschattung durch die APV-Reihen auf die Entwicklung der darunter liegenden Pflanzen hat. Dafür galt es, auf dem Testfeld jeweils $80 \%$ des Ertrages einer gleich großen PV-Freiflächenanlage (für Strom) und eines Referenzfeldes ohne APV (für Feldfrüchte) zu erzielen. Das Ergebnis war, dass die landwirtschaftlichen Erträge der angepflanzten Kulturen unter den APV-Reihen zwar etwas geringer ausfielen als ohne APV. Doch durch die im ersten Jahr gewonnenen rd. 1,3 MWh (1.266 kWh) Strom bei einer installierten Leistung von $194 \mathrm{~kW}$, wurde die Flächennutzungseffizienz um bis zu 60 \% erhöht (ISE, 2018a; ULRICH, 2018). Der Minderertrag der angepflanzten Kulturen setzte sich im Detail wie folgt zusammen: 5,3\% (Kleegras) und -18 bis -19\% für Stärkepflanzen (Kartoffeln, Sellerie und Weizen) im Vergleich zum Referenzstreifen. Auch in anderen Ländern gibt es Forschungsaktivitäten, in deren Rahmen teilweise auch schon wesentlich größere Installationen getestet wurden (TROMMSDORF, 2018; VITI, 2018). Das Fraunhofer-Institut für Solare Energiesysteme (ISE) hat jüngst damit begonnen, einen Technologietransfer in Entwicklungs- und Schwellenländer durchzuführen und an neuen Anwendungsfeldern für APV zu arbeiten (ISE, 2018b). Dafür wurden Kooperationen mit drei Gemeinden in Chile eingegangen, da das Potenzial für APV gerade in den ariden und semi-ariden Regionen Nord- und Zentralchiles als besonders groß eingeschätzt wird. So hilft die Teilbeschattung der landwirtschaftlichen Fläche durch APV-Anlagen den Wasserverbrauch zu reduzieren und bietet gleichzeitig schattigen Unterstand für etwaige Nutztiere. Die installierten APV-Anlagen sind die ersten ihrer Art in Lateinamerika.

Neben dem großen Potenzial einer effizienteren Landnutzung durch den mithilfe von APV erzielten „doppelten Ertrag“ (PV-Strom und Feldfrüchte), ist eine Vielzahl von weiteren Synergieeffekten denkbar. Gerade in rural geprägten Gegenden in Deutschland aber auch global betrachtet, können mit APV bebaute Flächen mittelfristig zu einer höheren Wertschöpfung führen und gleichzeitig sensiblen Nutzpflanzen Schutz vor Wetterextremen, wie Dürre, Frost und Hagel bieten (NIEPELT, 2018). Daneben wären auch Konzepte zur Eindämmung einer fortschreitenden Desertifikation denkbar (TROMMSDORF, 2018). Denn 
APV-Flächen liefern nicht nur Schatten, sondern erzeugen auch Strom, der zur Bewässerung oder zur Aufbereitung von Grundwasser in ariden Regionen genutzt werden kann.

$\mathrm{Zu}$ der Feld überspannenden Bauweise gibt es auch die Alternative, bifaziale Photovoltaikmodule senkrecht aufzustellen (RÖPCKE, 2018; MERTENS, 2018). Auf diese Weise können die Module das von beiden Seiten aufgefangene Sonnenlicht in elektrische Energie umwandeln. Daneben wohnt solchen Konstruktionen der Vorteil inne, dass zwischen den zaunartig stehenden APV-Reihen die landwirtschaftliche Nutzung nur geringfügig eingeschränkt wird. In Zusammenarbeit mit dem Unternehmen Next2Sun GmbH entsteht im Saarland auf rd. 10 Hektar Ackerland derzeit der größte bifaziale Solarpark Europas (rd. 2 MW peak) (NEXT2Sun, 2018; WochENSPIEGEL, 2018). Im August 2018 wurde am Standort Dirmingen (Saarland) bereits der erste Wechselrichter der Großanlage in Betrieb genommen.

In Deutschland bestehen zurzeit noch eine Reihe rechtlicher Hürden, die einer wirtschaftlichen Attraktivität von APV-Anlagen im Weg stehen. Denn diese Form der Flächennutzung ist (noch) nicht Teil des Erneuerbare-Energien-Gesetzes (EEG), womit eine erhöhte Vergütung für den auf diese Weise gewonnenen Strom entfällt. Darüber hinaus gelten landwirtschaftliche Flächen, die mit APV bebaut werden als Sondernutzungsflächen, für die kein Anspruch auf europäische Agrarsubventionen im Rahmen der Gemeinsamen Agrarpolitik (GAP) erhoben werden kann. Es ist allerdings davon auszugehen, dass das hohe Potenzial zur Entschärfung von Flächenkonkurrenzen sowie die Möglichkeit einer Ertragssteigerung bei Feldfrüchten durch ein Abmildern von Extremwettererscheinungen, was vor allem für südliche EU-Mitgliedstaaten, in extrem trockenen Jahren (wie 2018) auch für Deutschland von Relevanz ist, zu einer mittelfristigen Entschärfung der benannten rechtlichen Hürden führen. Daneben ist der mithilfe von APV erzeugte Strom deutlich teurer (Gestehungskosten: 12 bis $16 \mathrm{ct} / \mathrm{kWh}$ ) als Strom aus herkömmlichen Standardmodulen und somit entsprechend schwieriger zu vermarkten (TROMMSDORF, 2018). Eine deutlich gröBere Attraktivität kann die Eigennutzung von APV-Strom sein, da für eine Wirtschaftlichkeitsbetrachtung in diesem Fall der erhöhte Endkundenpreis als Referenz dient (ibid.). Denkbar wäre auch die Nutzung des auf diese Weise gewonnenen Stroms in elektrisch betriebenen stationären und mobilen Land- bzw. Kleinmaschinen. Denn im Zuge der fortschreitenden Energiewende wird die Sektorenkopplung auch in der Landwirtschaft an Wert gewinnen.

Wirft man einen Blick auf die fortschreitende Entwicklung des APV-Konzepts, fällt auf, dass das Thema in jüngerer Zeit an Relevanz gewonnen hat (Abbildung 9). 


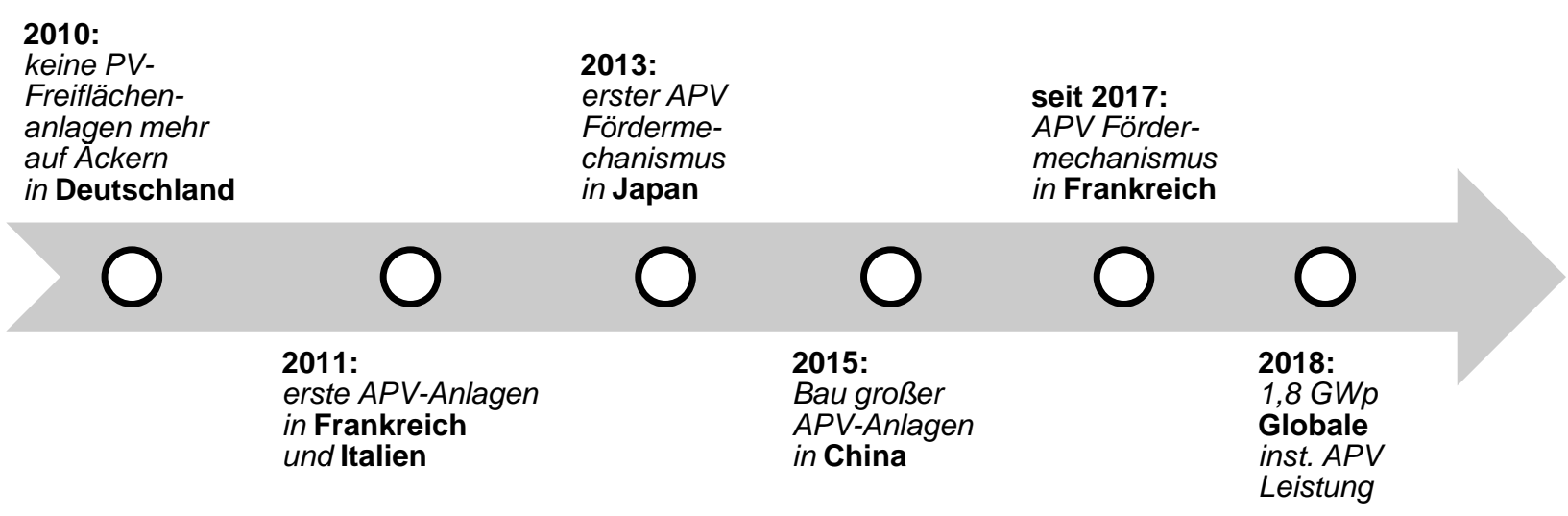

Abbildung 9: Zeitstrahl Entwicklung von Agrarphotovoltaik-Anlagen

Quelle: Eigene Darstellung nach TROMMSDORF (2018)

In Japan existiert bereits seit 2013 ein Fördermechanismus für APV-Anlagen, dessen Beispiel Frankreich, als Vertreter eines europäischen Staates, im Jahr 2017 gefolgt ist (MoVELLAN, 2013). Man hat dies dort nach erfolgreichen Feldversuchen durchsetzen können. So wurden beispielsweise an der Universität von Montpellier an einer kleinen APV-Anlage verschiedene Untersuchungen durchgeführt, die bestätigten, dass die Erträge einer lichtliebenden Pflanze, wie Weizen, unter der Beschattung durch die APV-Anlage geringer ausfielen als in der nicht beschatteten Kontrollgruppe (DUPRAZ et al., 2011). In einer daran angeschlossenen Studie konnten dieselben Forscher bereits ein Jahr später bestätigen, dass eine solche Teilbeschattung für die Ertragsentwicklung bestimmter Salatsorten hingegen von Vorteil sein kann (MARROU et al., 2013a; MARROU et al., 2013b). Eine mögliche Gestaltung einer APV-Regulierung in Deutschland wurde im August 2014 vom Fraunhofer ISE und der Wuppertal Institut für Klima, Umwelt, Energie GmbH (Wuppertal Institut) im Zuge einer Stellungnahme erörtert und aufgezeigt, aus welchen Gründen eine solche notwendig sei (ISE und WUPPERTAL INSTITUT, 2014). 


\section{Literatur}

BAYRISCHES LANDWIRTSCHAFTLICHES WOCHENBLATT (2017): Landwirte vermehrt bei Windkraft und Photovoltaik aktiv. URL: https://www.agrarheute.com/wochenblatt/feld-stall/energie/landwirte-vermehrt-windkraft-photovoltaik-aktiv-540183 (Abrufdatum: 15.01.2019).

BDBE (Bundesverband der deutschen Bioethanolwirtschaft e.V.) (2018): Die deutsche Bioethanolwirtschaft in Zahlen. URL: https://www.bdbe.de/daten/marktdaten-deutschland (Abrufdatum:10.01.2019).

BWK (Brennstoff, Wärme, Kraft - Das Energie-Fachmagazin) (2018): Erneuerbare Energien weltweit. URL: https://www.energiefachmagazin.de/2017/Ausgabe-7-8/Special-Erneuerbare-Energien/ErneuerbareEnergien-weltweit?page $=14$ (Abrufdatum: 06.01.2019).

BMEL (Bundesministerium für Ernährung und Landwirtschaft) (2017): Waldbericht der Bundesregierung 2017. BMEL, Bonn.

BMEL (Bundesministerium für Ernährung und Landwirtschaft) (2018): Erntebericht 2018. URL: https://www.bmel.de/DE/Landwirtschaft/Pflanzenbau/Ackerbau/_Texte/Ernte2018.html (Abrufdatum: 14.01.2019).

BMJV (Bundesministerium der Justiz und für Verbraucherschutz) (2018a): Energiesteuergesetz. URL: https://www.gesetze-im-internet.de/energiestg/ (Abrufdatum: 10.12.2018).

BMJV (Bundesministerium der Justiz und für Verbraucherschutz) (2018b): Verordnung über den Umgang mit Nährstoffen im Betrieb und betriebliche Stoffstrombilanzen URL: https://www.gesetze-im-internet.de/stoffbilv/ (Abrufdatum: 10.12.2018).

BMJV (Bundesministerium der Justiz und für Verbraucherschutz) (2018c): Bundeswaldgesetz. URL: https://www.gesetze-im-internet.de/bwaldg/ (Abrufdatum: 14.11.2018).

BMJV (Bundesministerium der Justiz und für Verbraucherschutz) (2018d): Bundesnaturschutzgesetz. URL: https://www.gesetze-im-internet.de/bnatschg_2009/BJNR254210009.html (Abrufdatum: 14.11.2018).

BMU (Bundesministerium Umwelt, Naturschutz und nukleare Sicherheit) (2018a): Svenja Schulze zu Hilfen für von der Dürre bedrohte Landwirte. URL: https://www.bmu.de/meldung/svenja-schulze-zu-hilfenfuer-von-der-duerre-bedrohte-landwirte/ (Abrufdatum: 14.01.2019).

BMU (Bundesministerium Umwelt, Naturschutz und nukleare Sicherheit) (2018b): Klimaschutz in Zahlen: Klimaschutzziele Deutschland und EU. URL: https://www.bmu.de/fileadmin/Daten_BMU/Download_PDF/Klimaschutz/klimaschutz_in_zahlen_klimaziele_bf.pdf (Abrufdatum: 14.01.2019).

BMWI (Bundesministerium für Wirtschaft und Energie) (2018a): Die nächste Phase der Energiewende: Das EEG 2017. URL: https://www.bmwi.de/Redaktion/DE/Artikel/Energie/eeg-2017-start-in-dienaechste-phase-der-energiewende.html (Abrufdatum: 14.01.2019).

BMWI (Bundesministerium für Wirtschaft und Energie) (2018b): Zeitreihen zur Entwicklung der erneuerbaren Energien in Deutschland - Stand: August 2018. URL: https://www.erneuerbare-energien.de/EE/Redaktion/DE/Downloads/zeitreihen-zur-entwicklung-der-erneuerbaren-energien-in-deutschland-1990-2017.pdf;jsessionid=653852D5BF3FFAE0A5D117517766EC37? _blob=publicationFile\&v=16 (Abrufdatum: 05.12.2018).

BMWI (Bundesministerium für Wirtschaft und Energie) (2018c): Erneuerbare Energien in Zahlen: Nationale und internationale Entwicklung im Jahr 2017. URL: https://www.erneuerbare-energien.de/EE/Redaktion/DE/Downloads/Berichte/erneuerbare-energien-in-zahlen-2017.pdf?_blob=publicationFile\&v $=7$ (Abrufdatum: 10.12.2018).

BMWI (Bundesministerium für Wirtschaft und Energie) (2018d): GWS Research Report 2018/01: Ökonomische Indikatoren des Energiesystems - Methode, Abgrenzung und Ergebnisse für den Zeitraum 2000 2016. URL: https://www.bmwi.de/Redaktion/DE/Publikationen/Studien/oekonomische-indikatoren-und-energiewirtschaftliche-gesamtrechnung.pdf?_blob=publicationFile\&v=16 (Abrufdatum: 10.12.2018). 
BOHNET, S., HAAK, F., THRÄN, D. und T. SCHMIDT-BAUM (2017): Effiziente Bioenergie für Regionen - Ergebnisse der technisch-ökonomischen Begleitforschung zur Fördermaßnahme Bioenergie-Regionen 2012-2015 (DBFZ Report Nr. 29). DBFZ, Leipzig.

Bolte, A., Börner, J., Bräsicke, N., Degen, B., Dieter, M., SAAke, B. und B. U. SChNeider (2016): Perspektiven der Forst- und Holzwirtschaft in Deutschland. URL: http://biooekonomierat.de/fileadmin/Publikationen/berichte/Hintergrundpapier_Forstwirtschaft_280416_final.pdf (Abrufdatum: 06.12.2018).

BunZEL, K., THRÄN, D., SEYFERT, U., ZELLER, V. und M. BUCHHORN (2011): Forstwirtschaftliche Biomassepotenziale und Rohstoffpotenziale in Deutschland. In: BBSR und BBR (Hrsg.): Biomasse: Perspektiven räumlicher Entwicklung 5/6: 297-308.

DeSTATIS (2018): Bodenfläche insgesamt nach Nutzungsarten in Deutschland am 31.12.2017. URL: https://www.destatis.de/DE/ZahlenFakten/Wirtschaftsbereiche/LandForstwirtschaftFischerei/Flaechennutzung/Tabellen/Bodenflaeche_Insgesamt.html (Abrufdatum: 10.12.2018).

DIE ZEIT (2018): Die Zukunft im Schwitzkasten. URL: https://www.zeit.de/2018/32/klimawandel-globaleerwaermung-hochsommer-duerre-wetter (Abrufdatum: 14.01.2019).

Dotzauer, M., Pfeiffer, D., Thrän, D., Lenz, V., Pohl, M. und F. MÜller-Langer (2018): Technologiebericht 1.1 Bioenergie. In: Wuppertal Institut, ISI, IZES (Hrsg.): Technologien für die Energiewende. Teilbericht 2 an das Bundesministerium für Wirtschaft und Energie (BMWi). Wuppertal, Karlsruhe, Saarbrücken.

DritTler, L., RÜBCKE VON Veltheim, F., SCHAPER C. Und L. THEuvSEN (2018): Der Markt für Bioenergie. In: Die landwirtschaftlichen Märkte an der Jahreswende 2017/18 67 (2018), Supplement: 102-118.

Dupraz, C., Mattou, H., Talbot, G., Dufour, L., Nogier, A. und Y. Ferard (2011): Combining solar photovoltaic panels and food crops for optimising land use: Towards new agrivoltaic schemes. In: Renewable Energy 36 (2011): 2725-2732.

EPURE - (2018): European renewable ethanol - key figures 2017. URL: https://www.epure.org/media/1763/180905-def-data-epure-statistics-2017-designed-version.pdf (Abrufdatum: 06.01.2019).

EUROPEAN COMMISSION (2018): $\mathrm{CO}_{2}$-arme Wirtschaft bis 2050. URL: https://ec.europa.eu/clima/policies/strategies/2050_de\#tab-0-0 (Abrufdatum: 06.01.2019).

FNR (Fachagentur für Nachwachsende Rohstoffe) (2014): Leitfaden Bioenergie. Planung, Betrieb und Wirtschaftlichkeit von Bioenergieanlagen. Gülzow.

FNR (Fachagentur für Nachwachsende Rohstoffe) (2015): Biomassepotenziale von Rest- und Abfallstoffen. Status quo in Deutschland. Gülzow.

FNR (Fachagentur für Nachwachsende Rohstoffe) (2018a): Anbau und Verwendung nachwachsender Rohstoffe in Deutschland. URL: https://mediathek.fnr.de/grafiken/daten-und-fakten/stoffliche-einsatzmengen-nachwachsender-rohstoffe-in-deutschland.html (Abrufdatum: 10.12.2018)

FNR (Fachagentur für Nachwachsende Rohstoffe) (2018b): Maisanbau in Deutschland. URL: https://mediathek.fnr.de/grafiken/daten-und-fakten/bioenergie/biogas/maisanbau-in-deutschland.html (Abrufdatum: 10.12.2018).

FNR (Fachagentur für Nachwachsende Rohstoffe) (2018c): Entwicklung Biodiesel-Produktion und -Absatz in Deutschland. URL: https://mediathek.fnr.de/grafiken/daten-und-fakten/bioenergie/biokraftstoffe/entwicklung-biodiesel-in-deutschland.html (Abrufdatum: 10.12.2018).

FNR (Fachagentur für Nachwachsende Rohstoffe) (2018d): Stromerzeugung aus Biomasse. In: https://mediathek.fnr.de/stromerzeugung-aus-biomasse.html (Abrufdatum: 30.11.2018).

FNR (Fachagentur für Nachwachsende Rohstoffe) (2018e): Festbrennstoffe. In: https://bioenergie.fnr.de/bioenergie/festbrennstoffe/ (Abrufdatum: 30.11.2018).

FNR (Fachagentur für Nachwachsende Rohstoffe) (2018f): Basisdaten Bioenergie Deutschland 2018. Gülzow. URL: http://www.fnr.de/fileadmin/allgemein/pdf/broschueren/Broschuere_Basisdaten_Bioenergie_2018_web.pdf (Abrufdatum: 14.01.2019). 
FrANKFURT SCHOOL-UNEP CENTRE/BNEF (Bloomberg New Energy Finance) (2018): Global Trends in Renewable Energy Investment 2017, Key Messages GTR 2017. URL: http://www.iberglobal.com/files/2018/renewable_trends.pdf (Abrufdatum: 11.12.2018).

FvB (Fachverband Biogas e.V.) (2018): Branchenzahlen 2017 und Prognose der Branchenentwicklung 2018, Stand: Mai 2018. URL: https://www.biogas.org/edcom/webfvb.nsf/id/DE_Branchenzahlen/\$file/1805-25_Biogas_Branchenzahlen-2017_Prognose-2018_end.pdf (Abrufdatum: 11.12.2018).

Gömann, H., Bender, A., Bolte, A., Dirksmeyer, W., Englert, H., Feil, J.-H., Frühauf, C., HauSCHILD, M., KRENGEl, S., Lilienthal, H. LÖPMEIER, F.-J., MÜller, J., MußHOfF, O., NATKHin, M., Offermann, F., Seidel, P., Schmidt, M., Seintsch, B., Steidl, J., Strohm, K. und Y. Zimmer (2015): Agrarrelevante Extremwetterlagen und Möglichkeiten von Risikomanagementsystemen: Studie im Auftrag des Bundesministeriums für Ernährung und Landwirtschaft (BMEL). URL: https://www.bmel.de/SharedDocs/Downloads/Landwirtschaft/Klima-und-Umwelt/Klima-

schutz/AbschlussberichtProjektExtremwetterlagen.pdf?_blob=publicationFile (Abrufdatum: 14.01.2019).

IPCC (Intergovernmental Panel on Climate Change) (2014): Climate Change 2014: Mitigation of Climate Change. Contribution of Working Group III to the Fifth Assessment Report of the Intergovernmental Panel on Climate Change. Cambridge University Press, Cambridge und New York.

IRENA (International Renewable Energy Agency) (2018): Renewable Energy and Jobs - Annual Review 2018. URL: https://irena.org/-/media/Files/IRENA/Agency/Publication/2018/May/IRENA_RE_Jobs_Annual_Review_2018.pdf (Abrufdatum: 11.12.2018).

ISE (Fraunhofer-Institut für Solare Energiesysteme) (2018a): Sonne ernten auf zwei Etagen - Agrophotovoltaik steigert die Landnutzungseffizienz um über 60 Prozent. URL: https://www.ise.fraunhofer.de/de/presse-und-medien/presseinformationen/2017/sonne-ernten-auf-zwei-etagen-agrophotovoltaik-steigert-landnutzungseffizienz-um-ueber-60-prozent.html (Abrufdatum: 13.12.2018).

ISE (Fraunhofer-Institut für Solare Energiesysteme) (2018b): Presseinformation: Agrophotovoltaik goes global: von Chile bis Vietnam. URL: https://www.ise.fraunhofer.de/content/dam/ise/de/documents/presseinformationen/2018/1818_ISE_d_PI_APV_international.pdf (Abrufdatum: 14.12.2018).

ISE (Fraunhofer-Institut für Solare Energiesysteme) und WUPPERTAL INSTITUT (Wuppertal Institut für Klima, Umwelt, Energie GmbH) (2014): Stellungnahme zur BMWi-Konsultation „Eckpunkte für ein Ausschreibungsdesign für Photovoltaik-Freiflächenanlagen.“. URL: https://epub.wupperinst.org/frontdoor/deliver/index/docId/5547/file/5547_APV.pdf (Abrufdatum: 14.01.2019).

KAltschmitT, M., ThräN, D. und J. PONITKA (2010): Holz als Energieträger - Möglichkeiten und Grenzen im Kontext von globalen Entwicklungen. In: Forst und Holz 12 (2010): 18-25.

KEMFERT, C. (2018): Die Hitzewelle zeigt: Höchste Zeit für einen konsequenten Klimaschutz! In: DIW-Wochenbericht 85 (32): 689ff.

KRÖGER, R., KONERDING, J. R. und L. THEUvSEN (2016): Identifikation von Einflussfaktoren auf die Nutzung von Güllefeststoffen als Gärsubstrat in Biogasanlagen. In: German Journal of Agricultural Economics 65 (2): 112-131.

Langenberg, J., DritTler, L., VON Bierbrauer, T., SCHAPer, C. und L. Theuvsen (2017): Der Markt für Bioenergie. In: German Journal of Agricultural Economics 66 (Supplement): 107-125.

LFU (Bayerisches Landesamt für Umwelt) (2018): Biogene Festbrennstoffe. URL: https://www.lfu.bayern.de/energie/biogene_festbrennstoffe/index.htm (Abrufdatum: 30.11.2018).

Marrou, H., Wery, J., Dufour, L. und C. Dupraz (2013a): Productivity and radiation use efficiency of lettuces grown in the partial shade of photovoltaic panels. In: European Journal of Agronomy 44 (2013): 54-66.

MARrou, H., DufOur, L. und J. WerY (2013b): How does a shelter of solar panels influence water flows in a soil-crop system? In: European Journal of Agronomy 50 (2013): 38-51. 
Mertens, K. (2018): Photovoltaik: Lehrbuch zu Grundlagen, Technologie und Praxis. Carl Hanser Verlag GmbH \& Co. KG, München.

Movellan, J. (2013): Japan Next-Generation Farmers Cultivate Crops and Solar Energy. URL: https://www.renewableenergyworld.com/articles/2013/10/japan-next-generation-farmers-cultivateagriculture-and-solar-energy.html (Abrufdatum: 14.01.2019).

MÜHLENHOfF, J. (2013): Reststoffe für Bioenergie nutzen - Potentiale, Mobilisierung und Umweltbilanz. Agentur für Erneuerbare Energien, Berlin.

MÜHLenhoff, J. und B. Dannemann (2017): Biogene Test- und Abfallstoffe. Flexibler Baustein der Energiewende. In: AEE (Agentur für Erneuerbare Energien) Renews Spezial 81.

NeXT2Sun (2018): Pressestimmen. URL: https://next2sun.de/\#Pressestimmen (Abrufdatum: 13.12.2018).

NIEPELT, R. (2018): „INSIDE: Integration von Solarenergie in die niedersächsische Energielandschaft“, Vortrag beim Gemeinsamen Ausschuss für regenerative Energien von Landvolk und WLV, 06.11.2018, Borken.

NiTSCH, J. (2017): Notwendige Zielsetzungen für ein nachhaltiges Energiesystem und möglicher Beitrag der Biomasse. In: MÜLLER, M.C.M. und G. WAGENER-LOHSE (Hrsg.): Nachhaltiges Energiesystem: Welche Bioenergie brauchen wir? Evangelische Akademie Loccum, Rehburg-Loccum: 13-31.

OECD-FAO (2018): Agricultural Outlook 2018 -2027. Biofuels. URL: http://www.fao.org/docrep/i9166e/i9166e_Chapter9_Biofuels.pdf (Abrufdatum: 11.01.2019).

Philipps, S. P., BeTT, A. W., RAU, B. und R. SChlatmanN (2018): Technologiebericht 1.3 Photovoltaik. In: WUPPERTAL InSTITUT, ISI, IZES (Hrsg.): Technologien für die Energiewende. Teilbericht 2 an das Bundesministerium für Wirtschaft und Energie (BMWi), Wuppertal, Karlsruhe, Saarbrücken. URL: https://epub.wupperinst.org/frontdoor/deliver/index/docId/7043/file/7043_Photovoltaik.pdf (Abrufdatum: 12.12.2018).

PutTKAMmer, J. und H. GRETHE (2015): The public debate on biofuels in Germany: Who drives the discourse? In: German Journal of Agricultural Economics (GJAE), Special Issue: The Political Economy of the Bioeconomy 64 (4) (2015): 263-273.

REITER, G. und J. LINDORFER (2015): Global warming potential of hydrogen and methane production from renewable electricity via power-to-gas technology. In: The International Journal of Life Cycle Assessment 20 (4): 477-489.

REN21 (2018): Renewables 2018 Global Status Report. Paris. URL: http://www.ren21.net/wp-content/uploads/2018/06/17-8652_GSR2018_FullReport_web_final_.pdf (Abrufdatum: 10.12.2018).

RFA (Renewable Fuels Association) (2019): World Fuel Ethanol Production. URL: https:// ethanolrfa.org/resources/industry/statistics/ (Abrufdatum: 20.12.2017).

RÖPCKE, I. (2018): Neue Technologien für mehr Leistung. In: agrarheute - Energie 04 (2018).

SChaumanN, G. und K. W. SCHMitZ (2010): Kraft-Wärme- Kopplung. Springer, Berlin/Heidelberg.

SDW (Schutzgemeinschaft Deutscher Wald) (2018a): Waldanteil in Deutschland. URL: https://www.sdw.de/waldwissen/wald-in-deutschland/waldanteil (Abrufdatum: 14.11.2018).

SDW (Schutzgemeinschaft Deutscher Wald) (2018b): Holzvorrat. URL: https://www.sdw.de/waldwissen/wald-in-deutschland/holzvorrat/index.html (Abrufdatum: 14.11.2018).

SDW (Schutzgemeinschaft Deutscher Wald) (2018c): Besitzstruktur. URL: https://www.sdw.de/waldwissen/wald-in-deutschland/besitzstruktur/index.html (Abrufdatum: 14.11.2018).

SPIEgEL OnLine (2018): Hitze in Deutschland - „Es wird immer mehr Dürren geben“. URL: http://www.spiegel.de/wissenschaft/natur/hitze-in-deutschland-es-wird-immer-mehr-duerren-gebena-1220516.html (Abrufdatum: 14.01.2018).

STATISTA (2018a): Primärenergieverbrauch in Deutschland nach Energieträger in den Jahren 2016 und 2017. URL: https://de.statista.com/statistik/daten/studie/251493/umfrage/energieverbrauch-in-deutschland-nach-energietraeger/ (Abrufdatum: 05.12.2018). 
STATISTA (2018b): Anzahl der Biogasanlagen in Deutschland in den Jahren 1992 bis 2017. URL: https:/ de.statista.com/statistik/daten/studie/167671/umfrage/anzahl-der-biogasanlagen-in-deutschland-seit-1992/ (Abrufdatum: 05.12.2018).

STATISTA (2018c): Installierte elektrische Leistung der Biogasanlagen in Deutschland in den Jahren 1999 bis 2018 (in Megawatt). URL: https://de.statista.com/statistik/daten/studie/167673/umfrage/installierteelektrische-leistung-von-biogasanlagen-seit-1999/ (Abrufdatum: 05.12.2018).

STATISTA (2018d): Anteil Erneuerbarer Energien am Bruttostromverbrauch in Deutschland in den Jahren 1990 bis 2017. URL: https://de.statista.com/statistik/daten/studie/2142/umfrage/erneuerbare-energien-anteil-am-stromverbrauch/ (Abrufdatum: 05.12.2018).

STATISTA (2018e): Weltweiter Primärenergieverbrauch in den Jahren von 1980 bis 2017 (in Millionen Tonnen Öläquivalent). URL: https://de.statista.com/statistik/daten/studie/42226/umfrage/welt-insgesamt--verbrauch-an-primaerenergie-in-millionen-tonnen-oelaequivalent/ (Abrufdatum: 10.12.2018).

STATISTA (2018f): Wichtigste Länder weltweit nach installierter Leistung von Enneuerbare-Energien-Anlagen im Jahr 2017 (in Gigawatt). URL: https://de.statista.com/statistik/daten/studie/152750/umfrage/erneuerbare-energie---kapazitaet-in-gigawatt-2009/ (Abrufdatum: 11.12.2018).

STATiSTA (2018g): Anzahl neu zugelassener Pkw mit Dieselmotor in Deutschland in den Jahren 1990 bis 2017. URL: https://de.statista.com/statistik/daten/studie/184465/umfrage/zugelassene-diesel-pkw-indeutschland/ (Abrufdatum: 10.12.2018).

STATISTA (2018h): Anzahl neu zugelassener Pkw mit Benzinmotor in Deutschland in den Jahren 2007 bis 2017. URL: https://de.statista.com/statistik/daten/studie/818600/umfrage/pkw-mit-benzinmotor-neuzulassungen-in-deutschland/ (Abrufdatum: 10.12.2018).

STATISTA (2019): Wichtigste Länder weltweit nach Produktionsmenge von Biodiesel im Jahr 2017 (in Milliarden Liter). URL: https://de.statista.com/statistik/daten/studie/172713/umfrage/biodieselproduktionnach-ausgewaehlten-laendern-weltweit/ (Abrufdatum: 08.01.2019).

SÜDDEUTSCHE ZEITUNG (2018): Vorgeschmack auf die Sommer der Zukunft. URL: https://www.sueddeutsche.de/wissen/klimawandel-europa-duerre-1.4072135 (Abrufdatum: 14.01.2019).

TROMmSDORF, M. (2018): „Agrophotovoltaik - Ein Beitrag zur ressourceneffizienten Landnutzung““, Vortrag auf den Niedersächsischen Solarenergietagen, 06.09.2018, Hannover.

UfOP (Union zur Förderung von Oel- und Proteinpflanzen e.V.) (2018): Biodiesel 2017/2018 Sachstandsbericht und Perspektive - Auszug aus dem UFOP-Jahresbericht 10 (2018).

UlRICH, S. (2018): Doppelte Ernte. In: Erneuerbare Energien 7 (2018): 54-57.

UMWELTBUNDESAMT (2018): Primärenergieverbrauch. URL: https://www.umweltbundesamt.de/daten/energie/primaerenergieverbrauch\#textpart-3 (Abrufdatum: 05.12.2018).

UMWELTBUNDESAMT (2018a): Stromerzeugung erneuerbar und konventionell. URL: https://www.umweltbundesamt.de/daten/energie/stromerzeugung-erneuerbar-konventionell\#textpart-3 (Abrufdatum: 05.12.2018).

UMWELTBUNDESAMT (2018b): Emissionsbilanz erneuerbarer Energieträger. URL: https://www.umweltbundesamt.de/themen/klima-energie/erneuerbare-energien/erneuerbare-energien-in-zahlen\#emissionsbilanz (Abrufdatum: 30.11.2018).

USDA (U.S. Department of Agriculture) (2017): Gain Report. EU Biofuels Annual 2017. Nr. NL7015. URL: https://gain.fas.usda.gov/Recent\%20GAIN\%20Publications/Biofuels\%20Annual_The\%20Hague_EU-28_6-19-2017.pdf (Abrufdatum: 20.12.2017).

VDB (Verband der deutschen Biokraftstoffindustrie) (2019): Biodiesel. URL: http://www.biokraftstoffverband.de/index.php/biodiesel.html (Abrufdatum 20.12.2019).

VITI (2018): Un démonstrateur agrivoltaïque dans les Pyrénées-Orientales. URL: https://www.monviti.com/filinfo/viticulture/un-demonstrateur-agrivoltaique-dans-les-pyrenees-orientales (Abrufdatum: 13.12.2018). 
VOGELPOHL, T. (2018): Schlussbetrachtung. In: Biokraftstoffpolitik in Deutschland. Energiepolitik und Klimaschutz. Energy Policy and Climate Protection. Springer VS, Wiesbaden

WeLT (2018): Eine Dürre, wie es sie noch nie gab. URL: https://www.welt.de/wissenschaft/article181616914/Duerre-in-Deutschland-Was-der-trockene-Sommer-bewirkt-hat.html (Abrufdatum: 14.01.2019).

WOCHENSPIEGEL (2018): Bifaciale Solaranlage. URL: https://www.wochenspiegelonline.de/news/article/bifaciale-solaranlage/ (Abrufdatum: 13.12.2018).

Zschache, U., vON CRAMON-TAubadel, S. und L. Theuvsen (2010): Öffentliche Deutungen im Bioenergiediskurs. In: Berichte über Landwirtschaft 88 (3): 502-512. 


\section{Schlussbetrachtung und Ausblick}

Das Ziel der vorliegenden Dissertation war es, die Akzeptanz und die Nutzungsbereitschaft von AFR in der Landwirtschaft sowie die Sichtweise von Landtechnikherstellern auf diesen Prozess genauer zu untersuchen. Dies sollte einerseits ein umfassendes Verständnis des Akzeptanzprozesses selbst ermöglichen und andererseits die Abschätzung einer künftigen Etablierung von AFR in der Landwirtschaft erleichtern. Es wurden sowohl Potenziale als auch Bedenken dieser Technologie aufgezeigt. Somit können Stakeholder noch vor einer breiten Einführung von AFR entsprechend darauf reagieren. Andererseits bieten die Ergebnisse zahlreiche Anknüpfungspunkte für die Erforschung des Technologieakzeptanzprozesses in der Landwirtschaft allgemein. Schließlich soll die Arbeit einen Beitrag zur Entwicklung eines sowohl von Landtechnik als auch von Landwirten akzeptierten Konzepts zur erfolgreichen Etablierung von AFR in der Landwirtschaft leisten.

Während die Erforschung anderer PAT, wie GPS-gestützte Lenksysteme oder teilflächenspezifische Ausbringung von Dünger- bzw. Pflanzenschutzmitteln, wiederholt Gegenstand von Technikakzeptanzforschung waren, zeigte sich im ersten Teil dieser Arbeit, dass die Erforschung des Akzeptanzprozesses von AFR bisher kaum Beachtung fand (Beitrag I.2), was dem Umstand geschuldet sein kann, dass sich AFR aktuell in einer frühen Markteinführungsphase befinden. Potenziale werden für diese neue Technologie vor allem in der Bonitur bzw. Feldkartierung sowie der sensorischen Datenerfassung und Schädlingsbekämpfung gesehen. Dies berücksichtigend zeigten die Ergebnisse der Beiträge I.1 und I.3, dass Landwirte hinsichtlich ihrer Akzeptanz und Nutzungsbereitschaft elektrischer Antriebsysteme im Allgemeinen und AFR im Speziellen nicht als homogene Gruppe verstanden werden können, sondern sich unterschiedlich zum künftigen Einsatz solcher Technologien positionieren. Während die befragten Landwirte (Beitrag I.1) dem Einsatz von eLM nach Vorbild bisheriger Landmaschinen mehrheitlich kritisch gegenüberstanden, zeichnete sich beim Einsatz von AFR im Sinne von Kleinmaschinen bzw. Roboterschwärmen ein anderes Bild ab (Beitrag I.2). Hier ließen sich mehrere unterscheidbare Meinungsgruppen identifizieren. Auch wenn sich diese, als Ergebnis der Clusteranalyse festgelegten Gruppen in ihrer Nutzungsintention teils deutlich voneinander unterschieden, war eine über alle Gruppen hinweg neutrale bis positive Grundhaltung gegenüber AFR festzustellen, was mit den Ergebnissen von REDHEAD et al. (2015) übereinstimmt. Dieser vermeintliche Widerspruch zu der kritischen Einstellung von Landwirten hinsichtlich eLM allgemein lässt sich dahingehend entkräften, dass die Befragten den bloßen Ersatz von Verbrennungs- durch derzeitig verfügbare Elektromotoren in bestehender Landgroßtechnik für nicht praktikabel 
halten. Der neuartige, dem bisherigen „Wachstumsgesetz der Landtechnik“ entgegenstehende Ansatz einer Vielzahl elektrisch betriebener Kleinmaschinen, die in Schwärmen weitestgehend autonom zusammenarbeiten, erscheint hingegen künftig umsetzbarer. Generell kann mangelnde Erfahrung mit oder der Kenntnisstand über die Einsatzmöglichkeiten solcher Technologien zu Fehleinschätzungen führen und sich akzeptanzhemmend auswirken, was sowohl für AFR als auch eLM gilt (KAIRIES, 2013). Die drei gebildeten Gruppen (aufgeschlossene AFR-Unterstützer [rd. $52 \%$ ], überzeugte AFR-Anwender [rd. $31 \%$ ] und reservierte AFR Interessierte [rd. $17 \%$ ]) lassen sich in ihrer Nutzungsbereitschaft von AFR mit den ersten drei Anwender-Typen aus ROGERS“ (1995) Diffusionstheorie vergleichen. Dem folgend entsprechen die überzeugten AFR-Anwender den Innovatoren (,,innovators“), die aufgeschlossenen AFR-Unterstützer den frühen Anwendern („,early adopters") und die reservierten AFR-Anwender der frühen Mehrheit (,early majority“). Die drei Gruppen unterschieden sich neben ihrer AFR Nutzungsbereitschaft signifikant hinsichtlich ihrer Risikobereitschaft, ihrer strategischen Anpassungsfähigkeit und ihrem Willen, umweltfreundlicher zu wirtschaften. Der aus der Literatur abgeleitete und vorab angenommene Betriebsgrößenunterschied zwischen den einzelnen Clustern hingegen konnte nicht belegt werden.

Daneben wirkt eine Vielzahl von Akzeptanzfaktoren auf die Nutzungsbereitschaft von eLM (Beitrag I.1) bzw. AFR (Beiträge I.3 und I.4) unter Landwirten, die es für eine erfolgreiche Marktdurchdringung gezielt anzusprechen gilt. Für eLM konnte ein Einfluss der Faktoren Offenheit für Innovationen, Information, Technik, Umwelt, Preis, Politik und Einstellung bestätigt werden, während die Nutzungsintention von AFR signifikant und verschieden stark durch die Faktoren erwarteter Nutzen, erwarteter Aufwand, sozialer Einfluss, Technikbereitschaft, Angst und Vertrauen beeinflusst wurde. Unter ihnen wirkte der erwartete Nutzen (v.a. wirtschaftliche Aspekte betreffend) am stärksten und positiv auf die Nutzungsintention, was bei dem verwendeten UTAUT Modell oft der Fall ist (VENKATESH et al., 2003). Weitere, signifikant positive Wirkungen gingen in abschwächender Effektstärke vom sozialen Einfluss, der Technikaffinität (als Teil der Technikbereitschaft) und dem Vertrauen aus. Dagegen konnte für die Faktoren erwarteter Aufwand und Angst ein signifikant negativer Einfluss auf die Nutzungsintention von AFR nachgewiesen werden. Hier befürchteten die Landwirte zwar tendenziell Schwierigkeiten hinsichtlich einer fachgerechten Bedienung von AFR, doch konnte von einer Angst vor der Technologie im eigentlichen Sinne keinesfalls die Rede sein. Insgesamt zeichnete sich die Stichprobe durch eine hohe Technikbereitschaft aus und zeigte eine positive Tendenz v.a. in Bezug auf eine zu erwartende Reduzierung der Arbeitsbelastung durch AFR. Diese Erwartungshaltung wird zusätzlich durch die Ergebnisse von RELF-ECKSTEIN (2019) gestützt, nach denen sich 
der Gesundheitszustand von Landwirten nach Entlastung durch Roboter nachweislich verbessert hat. Die hohe Technikbereitschaft gilt allgemeinhin als gute Voraussetzung für eine künftige Technologieadoption und wurde oft für Landwirte bestätigt (u.a. AUSTIN et al., 1998; Voss et al., 2008)

Teil II dieser Arbeit hat gezeigt, dass viele der Erkenntnisse aus Teil I den Erwartungen der Landtechnikbranche entsprechen. So stimmten sowohl Landmaschinenhersteller (Beitrag II.1) als auch AgTech Startups (Beitrag II.2) dahingehend überein, dass eine Vielzahl von Faktoren die künftige Adoption von AFR beeinflusst, von denen die größte Wirkung vom erwarteten Nutzen ausgeht. Dabei wiegen aus ihrer Sicht die ökonomischen Vorteile deutlich schwerer als die ökologischen, was sich mit den Ergebnissen anderer Studien zur Akzeptanz von PAT deckt (u.a. PIERPAOLI et al., 2013; SALIMI et al., 2020). Den ökologischen Vorteilen wurde von den AgTech Startups ein eher indirekter Effekt zugesprochen, insofern, als dass konventionelle Landwirte mehr oder weniger gezwungen werden unter immer restriktiveren Vorgaben zu wirtschaften und ihre Produktionsweise ökologisch nachhaltiger zu gestalten, woraus sich eine extrinsische Motivation ergibt, Technologien wie AFR einzusetzen. Dasselbe gilt für den empfundenen soziopolitischen Druck, der auf die Landwirte u.a. in öffentlichen Debatten und den sich daraus ergebenden politischen Strömungen ausgeübt wird (siehe DöRR et al., 2019; FINGER et al., 2019). Dieses Empfinden wurde durch die Ergebnisse von Beitrag III.1 dahingehend bestätigt, dass die meisten der in der Studie befragten Konsumenten mit konventioneller Landwirtschaft eher negativ besetzte Begriffe assoziierten und in „bäuerlicher“ Landwirtschaft eine Art erstrebenswertes Gegenmodell zur ,industriellen“ Landwirtschaft sahen. Anders als die befragten AgTech Startups rechneten die etablierten Landmaschinenhersteller jedoch erst in frühestens zehn Jahren mit einer breiten Marktdurchdringung von AFR im konventionellen Pflanzenbau. Ein Übergang von bisheriger Landtechnik zu autonomen Systemen wird nicht ruckartig passieren, so die Experten, sondern das Ergebnis eines gleitenden Übergangs sein, in dessen Zwischenstufen die Kompatibilität von neuer mit alter Technik eine wichtige Rolle im Akzeptanzprozess spielen wird. Einig waren sich beide Gruppen auch, dass autonome Robotertechnologien zunächst in Pflanzenkulturen mit hoher Wertschöpfung, wie Sonderkulturen oder Reihenfrüchten (z.B. Zuckerrübe) Einzug finden werden, da hier einerseits ein höherer Arbeitskräftebedarf bei schlechter zu automatisierenden Arbeitsvorgängen besteht und andererseits höhere Deckungsbeiträge pro Hektar erzielt werden bzw. hohe Kostenaufwendungen beim Pflanzenschutz erforderlich sind, sodass sich der erhöhte Kapitalbedarf für AFR eher rechnet. Generell wurde in den beiden Studien bestätigt, dass der Arbeitskräftebedarf bzw. das jeweils regionale Ausmaß des Fachkräftemangels die 
Akzeptanz von AFR positiv beeinflussen kann, was auch die Befragung von RIAL-LOVERA (2018) in Kanada ergab. Gleichzeitig reflektierten die befragten AFR Startups (Beitrag II.2) die Sorge vieler Landwirte vor einer zu hohen Komplexität der Robotersysteme und der mangelnden Möglichkeit, diese ohne fremde Hilfe zu warten oder zu reparieren. Solche Bedenken konnten in den Beiträgen I.3 und I.4 nur in geringem Maß festgestellt werden. Weiter hängt die Geschwindigkeit einer breiteren Einführung von AFR und die Dynamik ihrer Verbreitung nach Ansicht der Landtechnikbranche derzeit vor allem von den rechtlichen Rahmenbedingungen ab. So ist Maschinenautonomie in einer unkontrollierten Umgebung noch immer mit zahlreichen rechtlichen Hürden verbunden, was zu „Notlösungen“ der AgTech Startups führt, die sich damit nach Aussage der Experten zum Teil in rechtlichen Grauzonen bewegen. Nach DörR et al. (2019) sind Staaten wie Australien, Japan und die USA in der Anpassung des rechtlichen Rahmens für das Inverkehrbringen autonomer Systeme weiter fortgeschritten als die Staaten Westeuropas, denen sie dahingehend eine Art Stillstand attestieren. In Deutschland existiert im Koalitionsvertag der aktuellen Regierung eine Absichtserklärung, sich der rechtlichen Situation des vollautomatisierten bzw. autonomen Fahrens annehmen zu wollen (CDU/CSU und SPD, 2018). Dieses Vorhaben wurde in Teilen mit einem Gesetzentwurf umgesetzt, der fahrerloses Fahren künftig in festgelegten Bereichen des öffentlichen Straßenverkehrs ermöglichen soll (DEUTSCHER BUNDESTAG, 2021).

Ebenso wichtig für die Verbreitung von AFR sind die strukturellen Voraussetzungen der Landwirtschaftsbetriebe und die Betriebsleitereigenschaften, da einerseits ein gewisses $\mathrm{Maß}$ an digitaler Infrastruktur gegeben sein muss und andererseits eine risikofreudige, technikaffine Betriebsleitung eher bereit ist technische Neurungen auszuprobieren als eine technik- und risikoaverse (DEVITT, 2018). Ein Einfluss des Altersunterschieds verschiedener Betriebsleitungen wie von CAFFARO und CAVALLO (2019) für PAT festgestellt wurde, konnte nicht bestätigt werden. Strukturell vorteilhaft und für die volle Entfaltung des ökologischen Mehrwerts von AFR bedeutsam ist hier vor allem die Möglichkeit des Einsatzes von Energie aus (betriebseigenen) regenerativen Energiequellen. Während die Bedeutung der erneuerbaren Energien nicht zuletzt vor dem Hintergrund sich häufender Extremwetterereignisse infolge des Klimawandels insgesamt wächst, befindet sich der Bioenergiesektor in einem Schrumpfungsprozess zugunsten von Wind- und Photovoltaikanlagen (Beitrag III.2).

Weniger einig waren sich die etablierten Landtechnikhersteller und die AgTech Startups bei der erwarteten Wirkung des Faktors Information. Während die etablierten Landmaschinenhersteller (Beitrag II.1) betonten, dass Landwirte ohnehin schon unzählige Daten über 
Smartphone Apps oder Precision Farming Anwendungen an die Hersteller übermitteln und das bei AFR voraussichtlich ähnlich sein wird, wiesen die AgTech Startups (Beitrag II.2) darauf hin, dass gerade in sensiblen Anwendungsbereichen, wie dem Weinbau, individuelle Verarbeitungsprozesse einen hohen Stellenwert genießen und kein Interesse der Landwirte daran besteht, diese Informationen mit Landtechnikherstellern oder gar anderen Landwirten zu teilen.

\section{Handlungsempfehlungen}

Auf Grundlage, der im Zuge dieser Arbeit gewonnenen Erkenntnisse lassen sich unterschiedliche Handlungsempfehlungen für Landtechnikhersteller und politische Entscheidungsträger ableiten, um auf diese Weise einer breiten, von den Landwirten weitestgehend akzeptierten Markteinführung und Verbreitung von AFR den Weg zu ebnen.

Auf Ebene der Landtechnikhersteller können viele der in den einzelnen Studien (Beiträge I. 2, I.3, I.4, II.1 und II.2.) offengelegten Akzeptanzfaktoren von AFR über eine verbesserte, transparentere Kommunikation der Vorteilhaftigkeit dieser neuen Technologie adressiert werden. Dies sollte vorzugsweise über von Landwirten präferierte Medien (z.B. Fachzeitschriften wie „top agrar“ oder soziale Netzwerke wie „Instagram“) (Beitrag I.2) oder über Landtechnikmessen und praxisnahe Fachforen (z.B. „DLG-Feldtage“) geschehen. Als zusätzliche Multiplikatoren können beispielsweise Landwirtschaftskammern oder landwirtschaftliche Beratungs- und Maschinenringe fungieren. Hierbei ist zu empfehlen, gezielter auf die Bedenken der Landwirte einzugehen und den Fokus zunächst auf ökonomische Aspekte, wie das Verhältnis von Kosten und Nutzen oder Finanzierungsmöglichkeiten zu legen und in einem zweiten Schritt ökologische Vorteile und mögliche Arbeitsentlastungen durch AFR aufzuzeigen. Daneben empfiehlt sich von Landwirten befürchtete Umstellungsrisiken durch Darlegung eines plausiblen Gesamtkonzepts entgegenzuwirken, das im Falle elektrisch betriebener AFR auch glaubwürdige Umweltbilanzen (v.a. in Bezug auf die Rohstoffbeschaffung für die Herstellung von Akkumulatoren) und technisch ausgereifte Maßnahmen zum Schutz betriebseigener Daten beinhaltet. Hierfür sollten bestehende Landtechnikunternehmen und Startups in Hinblick auf eine bessere Kompatibilität der unterschiedlichen AFR-Einzellösungen untereinander in aktiven Austausch treten und markenübergreifende Gesamtlösungen ermöglichen, was einer besseren Integration verschiedener AFR-Konzepte in bestehende Betriebsabläufe erleichtern würde. Um eine höhere Akzeptanz der Gesellschaft gegenüber modernen landwirtschaftlichen Praktiken zu bewirken, sollte eine Aufklärung der breiten Öffentlichkeit in Hinblick auf die Funktionsweise und die Potenziale von AFR erfolgen. Hierfür sollten auch kritische Stakeholder, wie sich in Umwelt-, Natur- und Klimaschutz engagierende NGOs, bewusst eingebunden 
werden. Als ein Schwerpunkt empfiehlt sich dabei die Kommunikation der Umweltvorteile und der verringerten Schadstoff- und Lärmemissionen von AFR, damit diese von der Gesellschaft auch wahrgenommen werden. Konkrete Kommunikationskanäle stellen u.a. Vorträge bzw. Vorführungen auf Schulbesuchen und in Einkaufszentren aber auch informative Kurzbeiträge im laufenden Fernsehprogramm dar. Daneben sind Führungen mit Schulklassen oder interessierten Erwachsenen auf AFR-Testbetrieben, ein Tag der offenen Tür, oder (Dauer-)Ausstellungen rund um das Thema AFR in der Landwirtschaft denkbar. Neben einer besseren Kommunikation sind praktische Vorführungen in der Landwirtschaft ein weiteres Mittel, um Landwirte von der Funktionsweise von AFR und deren Zuverlässigkeit zu überzeugen und auf diese Weise möglichen Bedenken zu begegnen. Diese können durch praktische Workshops erweitert bzw. durch Schulungen der Landwirte ergänzt werden, um die Sorge vor der Komplexität solcher Systeme zu lindern. Dabei ist es wichtig, dass die Bedienung von AFR so einfach wie möglich gehalten wird, da es nicht der Landwirt sein sollte, der sich an die Bedienung der Technologie anpassen muss, sondern die Technologie, die sich an die Bedienung durch den Landwirt anpassen sollte (VIK et al., 2019). In einer qualitativen Befragung begrüßten australische Landwirte die Möglichkeit, an der Prototypentwicklung von AFR mitwirken zu dürfen (REDHEAD et al., 2015). Eine solche Strategie wäre auch für die europäische AFR-Entwicklung ein vielversprechender Ansatz, da neben dem direkten Kundenfeedback, AFR auf diesem Weg auch an Bekanntheit innerhalb der Branche gewinnen würden.

Um den Übergang von bestehender zu vollautomatisierter bzw. autonomer Technik möglichst fließend zu gestalten (Beiträge I.1, II.1 und II.2), wäre eine Entwicklung über intelligente, elektrisch betriebene Anbaugeräte hin zu AFR denkbar. Daneben könnte einem von den Landwirten befürchteten Kontrollverlust von AFR durch eine zusätzliche Fernsteuerungsfunktion entgegengewirkt werden. Damit hätten Landwirte jederzeit die Möglichkeit korrigierend einzugreifen oder, falls nötig, ganz die Kontrolle der Maschine zu übernehmen. Ein weiterer technischer Aspekt betrifft die Laufzeit von Akkumulatoren. Beitrag I. 1 hat deutlich gemacht, dass diese für einen akzeptierten Einsatz in der Landwirtschaft deutlich ausgebaut werden sollte. Vor dem Hintergrund der Zuverlässigkeit und Arbeitssicherheit von AFR muss zu jedem Zeitpunkt die Möglichkeit bestehen, durch menschliche Intervention korrigierend einzugreifen oder sie abzuschalten.

Die Beiträge II. 1 und II. 2 haben verdeutlicht, dass im Falle einer größeren Verbreitung von AFR in der Landwirtschaft auch bestehende Geschäftsmodelle überdacht werden sollten. Mögliche Ansätze zielen beispielsweise auf das Ausgliedern bestimmter Arbeitsgänge an spezialisierte Lohnunternehmen oder Landmaschinenhersteller in Form eines 
cloudbasierten pay-per-use ${ }^{11}$ Modells. Gerade kleinere Landwirtschaftsbetriebe mit geringer Ressourcenausstattung könnten von solchen Lösungen profitieren, wenngleich diese eine höhere Abhängigkeit des Landwirts vom jeweiligen Dienstleister bedeuten würden. Damit einhergehend ist es zu empfehlen, für risikoaversere Landwirte (Beitrag I.3) ein umfassendes Versicherungs- bzw. garantiertes Serviceangebot anzubieten.

Die Handlungsempfehlungen für politische Entscheidungsträger zielen vorrangig auf eine Technologieförderung bzw. eine Verringerung des Risikos für Landwirte ab, auf AFR umzustellen. Generell stehen politischen Entscheidungsträgern eine Reihe von finanziellen Fördermechanismen zu Verfügung, die auch für die Verbreitung von AFR Anwendung finden könnten. Denkbar wäre an dieser Stelle ein finanzieller Ausgleich der Mehrkosten von AFR im Vergleich zu konventionellen Landmaschinen analog zum bestehenden Umweltbonus für Elektroautos (BMJV, 2020). Einige Bundesländer, wie Hessen (Programm: „Innovation und Zusammenarbeit in der Landwirtschaft und in ländlichen Gebieten sowie Digitalisierung in der Landwirtschaft“) und Bayern (Programm: „Bayerisches Sonderprogramm Landwirtschaft Digital (BaySL Digital)“), fördern die Anschaffung von AFR bereits mit bis zu 40 Prozent der zuwendungsfähigen Ausgaben (BMWI, 2021). Daneben könnte auch eine einzelbetriebliche Umsetzung von AFR-Konzepten durch die zweite Säule der Gemeinsamen Agrarpolitik co-finanziert werden. Ein solches Förderprogramm stellt beispielsweise die „Europäische Innovationspartnerschaft (EIP)“ dar. Hierbei schlieBen sich mehrere Partner u.a. aus der Landwirtschaft zu einer operationellen Gruppe zusammen, um gemeinsam an der Umsetzung einer ressourcenschonenderen und gleichzeitig wettbewerbsfähigeren Wirtschaftsweise zu arbeiten (EUROPÄISCHE KOMMISSION, 2021).

Besonders in den qualitativen Interviews mit Landmaschinenherstellern und AgTech Startups klang die Notwendigkeit eines rechtlichen Rahmens an, der den Einsatz vollautomatischer bzw. autonomer Systeme zulässt. Diese dürfen nach derzeitigem Stand in Deutschland nur unter Berücksichtigung der gesetzlich festgelegten Wahrnehmungsbereitschaftsund Übernahmepflichten eingesetzt werden, womit AFR ihr volles Potenzial nicht ausschöpfen können (DEUTSCHER BUNDESTAG, 2018). Hier empfiehlt sich, nicht zuletzt vor dem Hintergrund vollautomatisierter Kraftfahrzeuge auf den Straßen, zeitnahe einheitliche Regeln zu finden, die einen sicheren und ethisch vertretbaren Einsatz solcher Technologien

\footnotetext{
${ }^{11}$ Das Gerät wird nicht vom Landwirt erworben, sondern es wird eine nutzungsabhängige Gebühr entrichtet (analog zum Maschinen-Leasing). Die tatsächliche Nutzung wird dabei durch Sensoren überprüft und in einer Daten-Cloud zur Gebührenermittlung in Echtzeit verarbeitet.
} 
ermöglichen. Zur Klärung haftungsrechtlicher Fragen bei einem Unfall könnte etwa das Verbauen einer „Black Box“ analog zum Flugverkehr Abhilfe schaffen (BMWI, 2013).

Da die strukturellen Gegebenheiten der Landwirtschaftsbetriebe ebenfalls einen Einfluss auf das Ausmaß des künftigen Einsatzes von AFR haben, aber auch vor dem Hintergrund zukunftsfähiger ländlicher Räume, sollte der begonnene Netzausbau weiter vorangetrieben werden. Eine moderne, digitale Infrastruktur ist eine Grundvoraussetzung für das reibungslose Funktionieren von Technologien wie AFR (Beiträge I.1, II.1 und II.2). Gerade strukturschwächere Landwirtschaftsbetriebe mit geringerer Flächenausstattung könnten davon und von der Tatsache, dass AFR besser skalierbar sind als konventionelle Landgroßtechnik profitieren. Von zentraler Bedeutung ist dabei die Erforschung und Förderung von Nutzungskonzepten betriebseigen erzeugter Energie aus regenerativen Quellen vor Ort sowie die Einbindung von elektrisch betriebenen AFR in intelligente Stromnetze, beispielsweise als Zwischenspeicher für überschüssigen Strom. Als neuer innovativer Entwicklungsansatz für eine ressourceneffizientere Doppelnutzung des knappen Faktors Boden gelten beispielsweise APV-Anlagen, die derzeit in verschiedenen Pilotprojekten gefördert und erprobt werden (u.a. TROMMSDORF, 2018; VITI, 2018). In Niedersachsen wurde hierfür eine ausnahmsweise Errichtung von APV-Anlagen auf unbebauten Flächen, die einer landwirtschaftlichen Nutzung vorbehalten sind, in der neuesten Verordnung zur Änderung über das Landes-Raumnutzungsprogramm (LROP-VO) aufgenommen. APV-Anlagen würden es auch bei geringeren Abständen über dem Boden AFR ermöglichen, unter den teildurchlässigen Photovoltaikmodulen Landwirtschaft zu bereiten. Dabei ist die Möglichkeit der Errichtung von Ladestationen entlang der APV-Anlagen für elektrisch betriebene AFR besonders interessant.

\section{Limitationen und weiterer Forschungsbedarf}

Bei den einzelnen Beiträgen dieser Arbeit dürfen zur richtigen Interpretation der Ergebnisse, die mit den unterschiedlichen verwendeten empirischen Forschungsmethoden einhergehenden Limitationen nicht außer Acht gelassen werden.

Für die Beiträge I.1, II.1 und II.2, die qualitative Experteninterviews als Erhebungsinstrument verwendet haben, bestehen diese in erster Linie in ihrem explorativen, nicht repräsentativen Charakter. Durch die Auswahl weniger Individuen, die stellvertretend für die Grundgesamtheit befragt werden, kommt es zwangsläufig zu Über- oder Unterrepräsentationen bestimmter Regionen und Betriebsformen (Beitrag I.1) oder der Marktmacht und Erfolgsabhängigkeit von AFR der Befragten (Beiträge II.1 und II.2). So wäre es beispielsweise naheliegend, dass die befragten AgTech Startups (Beitrag II.2) eine optimistischere Sichtweise auf die Verbreitung von AFR in der Landwirtschaft haben als die etablierten 
Landmaschinenhersteller (Beitrag II.1). Darüber hinaus lässt sich hinterfragen, ob Landtechnikunternehmen überhaupt geeignet sind, den Akzeptanzprozess neuartiger Technik unter Landwirten in ausreichender Güte zu beurteilen. Auch kann ein gewisser Grad an Subjektivität bei der Zuordnung der getätigten Expertenaussagen zu den jeweiligen Akzeptanzfaktoren und deren Interpretation nicht ausgeschlossen werden.

Bei den Beiträgen, die sich auf quantitative Erhebungsinstrumente stützen (Beiträge I.3, I.4 und III.1) wurden die Hyperlinks zur jeweiligen Umfrage in der Regel per E-Mail an Landwirte geschickt. Dieses Verfahren birgt stets die Gefahr, dass nur solche Individuen dem Aufruf folgen, die ein großes Interesse an dem Thema haben (sowohl als Befürworter oder als Gegner der Technologie). Dieses Phänomen ist auch als Selbstselektion bekannt (JACOBS et al., 2009) und kann zu einem verfälschten Meinungsbild führen, da Randgruppen in der Stichprobe überproportional stark vertreten sein können. Durch das Verwenden einer 5-stufigen Likert Skala als Antwortoption kann nicht ausgeschlossen werden, dass das Antwortverhalten gerade bei Unwissen- oder Unentschlossenheit einer gewissen „Tendenz zur Mitte" (in diesem Fall 3 von 5) folgt oder Fragen beantwortet werden, obwohl sie schlichtweg nicht verstanden wurden (BORTZ und DÖRING, 2006). Selbiges gilt für das Phänomen der „sozialen Erwünschtheit“, bei dem die Befragten eher solche Antworten geben, die ihrer Meinung nach gesellschaftlich erwünscht sind und nicht zwangsläufig ihrer eigenen entsprechen. Eine weitere Limitation stellt die Tatsache dar, dass für die einzelnen Einflussfaktoren stets bestimmte Ausprägungen erfragt wurden, sodass das Antwortverhalten bei anderen Ausprägungen möglicherweise andere Ergebnisse gezeigt hätte. Dieses dürfte zwar durch Annahme formativer Messungen (Beitrag I.4) nicht der Fall sein, kann aber nicht grundsätzlich ausgeschlossen werden.

Die Stichprobe in den Beiträgen I.3 und I.4 ist jünger und wirtschaftet auf größeren Betrieben als die Grundgesamtheit deutscher Landwirte. Da AFR je nach Ausgestaltung jedoch gerade für kleinere Betriebe wirtschaftlich vorteilhaft sein können (LOWENBERG-DEBOER et al., 2020), kann es daher zu einer Unterschätzung der tatsächlichen Akzeptanz von AFR in der Landwirtschaft gekommen sein. Daneben handelt es sich bei den meisten AFRKonzepten um Prototypen, sodass davon ausgegangen werden kann, dass die befragten Landwirte bisher wenig oder keine Erfahrung mit AFR sammeln konnten. MELENHORST und BouwHUIS (2004) konnten nachweisen, dass Erfahrung mit technischen Innovationen einen Einfluss auf deren Akzeptanz hat. Dies und das Phänomen der Intention-Verhaltenslücke (RENNER et al., 2007) berücksichtigend, kann nicht ausgeschlossen werden, dass das tatsächliche Verhalten bei oder nach einer Kaufentscheidung von AFR anders ausfällt als in den Beiträgen I.3 und I.4 prognostiziert. Schließlich sollte bedacht werden, dass es in 
den Umfragen und Interviews stets um AFR im Allgemeinen ging. Da diese jedoch sehr vielfältig ausgestaltet sind, könnte auch die Einstellung der Landwirte typenabhängig unterschiedlich ausfallen.

Die vorliegende Arbeit bietet eine Reihe von Anknüpfungspunkten für weiterführende bzw. die vorhandenen Ergebnisse auf ihre Belastbarkeit prüfende Forschung. So wäre es interessant zu erfahren, ob sich die vorhandenen Ergebnisse in größeren, repräsentativen Stichproben bestätigen lassen und ob es regional (z.B. klein- vs. großstrukturierte Bundesländer), im europäischen Vergleich oder in Abhängigkeit der Betriebsform bzw. des Anbausystems (ökologisch, konventionell, hybrid) zu Unterschieden hinsichtlich der Nutzungsbereitschaft von AFR in der Landwirtschaft kommt. Da die Vermarktung von AFR noch in ihren Anfängen steckt, konnten bislang kaum oder nur wenig Marktdaten erhoben werden. Längerfristig läge ein spannendes Forschungsfeld in vergleichenden Gegenüberstellungen retrospektiver Zeitreihenanalysen der Akzeptanz oder des Erfolgs von AFR. Mit zunehmend verfügbaren Preisrelationen marktreifer AFR können auch ökonomische Vergleichsrechnungen bezüglich der Investition in AFR und konventionelle Maschinen vorgenommen werden, die über bisherige, zumeist auf Annahmen beruhende Studien aus dem angelsächsischen Sprachraum hinaus gehen. Daran anknüpfend könnte in Abhängigkeit des Einsatzes von AFR die Auswirkung auf die Wirtschaftlichkeit der Betriebe beispielsweise mithilfe paarweiser Zuordnungen („Matching“) berechnet werden. Aus Sicht der Landmaschinenhersteller aber auch in Hinblick auf mögliche Förderinstrumente erscheint die Erforschung der Zahlungsbereitschaft von Landwirten für AFR (z.B. anhand von diskreten Choice Experimenten) von Interesse. Ergänzend dazu könnten mit einer solchen Methode auch die genauen Umstände erforscht werden, unter denen kritische Landwirte bereit wären, AFR zu adaptieren. Ein weiterer interessanter Anknüpfungspunkt besteht in der Überprüfung der tatsächlichen Umweltbilanz von AFR, abhängig vom jeweiligen Typ bzw. der Antriebsart mithilfe einer Lebenszyklusanalyse (BAUMANN und TILLMAN, 2004).

Neben der Landwirtschaft selbst, geht von der Gesellschaft als Ganzes ein zunehmend gröBeres Interesse an landwirtschaftlichen Produktionsmethoden aus (ZANDER et al., 2013; ZÜHLSDORF et al., 2016). Dabei kann eine zu große gesellschaftliche Ablehnung von neuartigen Technologien deren Einführung verhindern, wie es beim geplanten Einsatz genveränderter Pflanzenkulturen in der Landwirtschaft der Fall war (FERRETTI und PAVONE, 2009) oder deren Verbreitung hemmen, wie im Fall von Biogasanlagen (DOBERS et al., 2015). Dies berücksichtigend und vor dem Hintergrund einer vergleichenden Gegenüberstellung mit den Ergebnissen aus Landwirtschaft und Landtechnik, bedarf die gesellschaftliche Wahrnehmung von AFR weiterer Forschung. Besonders interessant erscheint hierbei 
ein Vergleich der Wahrnehmung von AFR in Abhängigkeit verschiedener Ausführungen bzw. Designs.

\section{Ausblick}

Zusammenfassend kann festgehalten werden, dass die Arbeit ihr gesetztes Ziel, nämlich einen Beitrag zur empirischen Erforschung der Akzeptanz und Nutzungsbereitschaft von AFR in der Landwirtschaft zu leisten, grundsätzlich erreicht hat. Gleichzeitig kann die abschließende Beantwortung dieser Frage zum jetzigen Zeitpunkt nicht eindeutig erfolgen, da sowohl Umfang der erhobenen Stichproben als auch der Stand der Entwicklung bzw. die Einführung der Technologie dies nicht vollumfänglich zulässt. Dennoch geben die Ergebnisse in ihrem überwiegend positiven Tenor Anlass zur Zuversicht, dass AFR ihr landwirtschaftliches Potenzial mittel- bis langfristig zur Entfaltung bringen können. Kurzfristig ist dabei entscheidend, inwieweit politische Entscheidungsträger den rechtlichen Rahmen hierfür schaffen und die weitere Verbreitung solcher Technologien unterstuitzen. Derzeitige Pressemitteilungen aus dem Bundesministerium für Ernährung und Landwirtschaft (BMEL), wie etwa die Förderung von robotergestützter Bekämpfung unerwünschter Begleitvegetation im Zuckerrübenanbau, können in diesem Zusammenhang als positive Signale verstanden werden, die die Kompatibilität bestehender mit hochautomatisierter Technologie weiter fördern (BMEL, 2021). In Hinblick auf die konkrete Ausgestaltung von AFR-Konzepten dominieren derzeit zwar elektrisch betriebene Systeme, doch stehen diese in direkter Konkurrenz mit anderen Antriebsarten, wie Wasserstoff, synthetische- oder Biokraftstoffe. Hier bleibt abzuwarten, welche Antriebstechnologie sich letztlich durchsetzt. AFR sollten als Chance verstanden werden, durch eine umweltentlastende Intensivierung der Flächenbewirtschaftung die Kluft, die sich zwischen gesellschaftlichen Erwartungen und der landwirtschaftlichen Realität gebildet hat, zumindest in Teilen überbrücken zu können, um so der Zukunft gemeinsam und nicht getrennt entgegenzutreten. Schließlich verfügen AFR über das Potenzial das Betreiben kleiner und mittelgroßer Landwirtschaftsbetriebe ökonomisch attraktiver zu gestalten und sich somit ein Stück weit vom beschriebenen „Wachsen oder Weichen“ Trend abzusetzen.

\section{Literatur}

Austin, E. J., Willock, J., Deary, I. J., Gibson, G. J., Dent, J. B., Edwards-Jones, G. und O. Morgan (1998): Empirical models of farmer behavior using psychological, social, and economic variables - Part I: Linear modelling. In: Agricultural Systems 58 (2): 203-224.

Baumann, H. und A.-M. Tillman (2004): The Hitch Hiker's Guide to Lca: An Orientation in Life Cycle Assessment Methodology and Applications. Studentlitteratur, Lund. 
BMEL (Bundesministerium für Ernährung und Landwirtschaft) (2021): BMEL fördert gezielte Unkrautbekämpfung im Zuckerrübenanbau. URL: https://www.bmel.de/SharedDocs/Pressemitteilungen/DE/2021/22-optikult.html (Abrufdatum: 24.05.2021).

BMJV (Bundesministerium für Justiz und Verbraucherschutz) (2020): Richtline zur Förderung des Absatzes von elektrisch betriebenen Fahrzeugen (Umweltbonus). URL: https://www.bmwi.de/Redaktion/DE/Downloads/B/bekanntmachung-der-richtlinie-zur-foerderung-absatzes-von-elektrisch-betriebenen-fahrzeugen-umweltbonus.pdf?_blob=publicationFile\&v $=4$ (Abrufdatum: 23.05.2021).

BMWI (Bundesministerium für Wirtschaft und Technologie) (2013): Recht für funktionale Sicherheit in der Autonomik. URL: https://www.digitale-technologien.de/DT/Redaktion/DE/Downloads/Publikation/autonomik-Leitfaden2.pdf?_blob=publicationFile\&v=3 (Abrufdatum: 29.10.2020).

BMWI (Bundesministerium für Wirtschaft und Technologie) (2021): Förderdatenbank. URL: https:/ /www.foerderdatenbank.de/FDB/DE/Home/home.html (Abrufdatum:23.05.2021).

BORTZ, J. und N. DÖRING (2006): Forschungsmethoden und Evaluation für Human- und Sozialwissenschaftler. Springer, Berlin/Heidelberg.

CAfFaro, F. und E. Cavallo (2019): The Effects of Individual Variables, Farming System Characteristics and Perceived Barriers on Actual Use of Smart Farming Technologies: Evidence from the Piedmont Region, Northwestern Italy. In: Agriculture (MDPI) 9 (5): 111, doi: 10.3390/agriculture9050111.

CDU/CSU und SPD (2018): Koalitionsvertrag zwischen CDU, CSU und SPD. 19. Legislaturperiode. URL: https://www.bundesregierung.de/resource/blob/656734/847984/5b8bc23590d4cb2892b31c987ad67 2b7/2018-03-14-koalitionsvertrag-data.pdf?download=1 (Abrufdatum: 1.Juli 2020).

DEUTSCHER BUNDESTAG (2018): Autonomes und automatisiertes Fahren auf der Straße - rechtlicher Rahmen. URL: https://www.bundestag.de/resource/blob/562790/c12af1873384bcd1f8604334f97ee4b9/wd-7111-18-pdf-data.pdf (Abrufdatum: 23.05.2021).

DEUTSCHER BundeSTAG (2021): Entwurf eines Gesetzes zur Änderung des Straßenverkehrsgesetzes und des Pflichtversicherungsgesetzes - Gesetz zum autonomen Fahren. https://dserver.bundestag.de/btd/19/274/1927439.pdf (Abrufdatum: 24.07.2021)

DEviTT, S. K. (2018): Cognitive Factors that Affect the Adoption of Autonomous Agriculture. In: Farm Policy Journal 15 (2): 49-60.

Dobers, G. M., M. Oehlmann, U. Liebe und J. Meyerhoff (2015): Einstellungen und Präferenzen zum Ausbau Erneuerbarer Energien. Ökologisches Wirtschaften - Fachzeitschrift 30 (1): 16-17, doi: 10.14512/OEW300116.

Dörr, J., Fairclough, B., Henningsen, J., Jahić, J., Kersting, S., Menning, P., PePer, C. und F. SCholTEN-BusCHHOFF (2019): Scouting the Autonomous Agricultural Machinery Market. Fraunhofer IESE and Kleffmann Group, Kaiserlautern, Lüdinghausen.

EUROPÄISCHE KOMMISSION (2021): epi-agri. URL: https://ec.europa.eu/eip/agriculture/en (Abrufdatum: 09.08.2021).

Ferretti, M. P. und V. PAVONE (2009): What do civil society organisations expect from participation in science? Lessons from Germany and Spain on the issue of GMOs. In: Science \& Public Policy 36 (4): 287-299, doi: 10.3152/030234209X436527.

Finger, R., Swinton, S., El Benni, N. und E. WAlter (2019): Precision farming at the nexus of agricultural production and the environment. In: Annual Review of Resource Economics 11: 313-335, doi: 10.1146/annurev-resource-100518-093929.

Jacobs, B., Hartog, J. und W. Vijverberg (2009): Self-selection Bias in Estimated Wage Premiums for Earnings Risk. Empirical Economics 37 (2): 271-286, doi: 10.1007/s00181-008-0231-0.

KAIRIES, B. (2013): Marketing für Elektroautos. Akzeptanz als notwendige Bedingung für die Marktdurchdringung. Diplomica, Hamburg. 
LOWEnBerg-DeBoer, J., HuAng, I. Y., Grigoriadis, V. und S. BlackMORE (2020): Economics of robots and automation in field crop production. In: Precision Agriculture 21 (2020), doi: 10.1007/s11119-01909667-5.

MELENHORST, A.-S. und D. G. BOuWhuis (2004): When do older adults consider the internet? An exploratory study of benefit perception. In: Gerontechnology 3 (2): 89-101.

Pierpaoli, E., Carli, G., Pignatti, E. und M. Canavari (2013): Drivers of Precision Agriculture Technologies Adoption: A Literature Review. In: Procedia Technology 8 (2013): 61-69, doi: 10.1016/j.protcy.2013.11.010.

Redhead, F., Snow, S., Vyas, D., Bawden, O., Russell, R., Perez, T. und M. Brereton (2015): Bringing the Farmer Perspective to Agricultural Robots. In: Begole, B., KIM, J., InKPEN, K. und W. WoO (Hrsg.): Proceedings of the 33rd Annual ACM Conference Extended Abstracts on Human Factors in Computing Systems. Association for Computing Machinery, New York: 1067-1072, doi: 10.1145/2702613.2732894.

RENNER, B., SPIVAK, Y., KWON, S. und R. SCHWARZER (2007): Does age make a difference? Predicting physical activity of South Koreans. In: Psychology and Aging 22 (3): 482-493, doi: 10.1037/08827974.22.3.482.

Relf-Eckstein, J. E., Ballantyne, A. T. und P. W. B. Phillips (2019): Farming Reimagined: A case study of autonomous farm equipment and creating an innovation opportunity space for broadacre smart farming. In: NJAS - Wageningen Journal of Life Science 90-91: 100307, doi: 10.1016/j.njas.2019.100307.

Rial-LoverA, K. (2018): Agricultural Robots: Drivers, Barriers and Opportunities for Adoption. In: N. TREMBLAY (Hrsg.): Proceedings of the 14th International Conference on Precision Agriculture, Montreal, 2427 June 2018. The International Society of Precision Agriculture, Monticello Illinois.

Rogers, E. M. (1995): Diffusion of Innovations. Free Press, New York.

Salimi, M., Pourdarbani, R. und B. A. Nouri (2020): Factors Affecting the Adoption of Agricultural Automation Using Davis's Acceptance Model (Case Study: Ardabil). In: Acta Technologica Agriculturae 23 (1): 30-39, doi: 10.2478/ata-2020-0006.

TROMmSDORF, M. (2018): „Agrophotovoltaik - Ein Beitrag zur ressourceneffizienten Landnutzung“, Vortrag auf den Niedersächsischen Solarenergietagen, 06.09.2018, Hannover.

Venkatesh, V., Morris, M. G., Davis, G. B. und F. D. Davis (2003): User Acceptance of Information Technology: Toward a Unified View. In: MIS Quarterly 27 (3): 425-478, doi: 10.2307/30036540.

Vik, J., Stræte, E. P., Hansen, B. G. und T. NÆRLand (2019): The political robot - The structural consequences of automated milking systems (AMS) in Norway. In: NJAS - Wageningen Journal of Life Sciences 90-91 (2019): 100305, doi: 10.1016/j.njas.2019.100305.

VITI (2018): Un démonstrateur agrivoltaïque dans les Pyrénées-Orientales. URL: https://www.monviti.com/filinfo/viticulture/un-demonstrateur-agrivoltaique-dans-les-pyrenees-orientales (Abrufdatum: 13.12.2018).

Voss, J., Schaper, C., Spiller, A. und L. Theuvsen (2008): Innovationsverhalten in der deutschen Landwirtschaft - Empirische Ergebnisse am Beispiel der Biogasproduktion. Paper presented at the 48th Annual Conference, Bonn, Germany, 24-26 September 2008. German Association of Agricultural Economists (GEWISOLA), Brunswick, doi: 10.22004/ag.econ.52666.

Zander, K., Isermeyer, F., BÜrgelt, D., Christoph-Schulz, I. B., SAlamon, P. und D. Weible (2013): Erwartungen der Gesellschaft an die Landwirtschaft. Stiftung Westfälische Landwirtschaft, Münster.

ZÜHLSDOrF, A., Spiller, A., GAuly, S. und S. KÜHL (2016): Wie wichtig ist Verbrauchern das Thema Tierschutz? Präferenzen, Verantwortlichkeiten, Handlungskompetenzen und Politikoptionen. Zühlsdorf + Partner GbR, Berlin. 


\section{Veröffentlichungs- und Vortragsverzeichnis}

\section{Publikationen in referierten wissenschaftlichen Zeitschriften}

DritTler, L., RÜBCKE VON Veltheim, F., Schaper, C. und L. TheuVSEN (2018): Der Markt für Bioenergie. In: German Journal of Agricultural Economics 67 (2018), Supplement: 102-118.

RÜBCKe VON Veltheim, F., Schaper, C. und H. HeISE (2019): Die gesellschaftliche Wahrnehmung von bäuerlicher und industrieller Landwirtschaft. In: Austrian Journal of Agricultural Economics and Rural Studies 28 (22): 167-173.

RÜBCKe VON Veltheim, F., Deutsch, M., Beer, L., Schaper, C. und V. OTTER (2019):

Der Markt für Bioenergie. In: German Journal of Agricultural Economics 68 (2019), Supplement: 131-148.

RÜBCKE VON Veltheim, F., Theuvsen, L. und H. Heise (2019): Akzeptanz autonomer Feldroboter im Ackerbaueinsatz: Status quo und Forschungsbedarf. In: Berichte über Landwirtschaft 97 (3).

Deutsch, M., Mohrmann, S., Rübcke von Veltheim, F., Schaper, C. und V. OTTER (2020): Der Markt für Bioenergie. In: German Journal of Agricultural Economics 69 (2020), Supplement: 142-162.

RÜBCKE VON VeltheiM, F. und H. Heise (2020): The AgTech Startup Perspective to Farmers Ex Ante Acceptance Process of Autonomous Field Robots. In: Sustainability 12 (24): 10570.

RÜBCKE VON VELTHEIM, F. und H. HeISE (2021): German Farmers' Attitudes on Adopting Autonomous Field Robots: An Empirical Survey. In: Agriculture 11 (3): 216.

RÜBCKE VON Veltheim, F., Rube, A. A. und H. Heise (2021): Elektromobilität in der Landwirtschaft - Eine qualitative Analyse zur Nutzerakzeptanz. In: German Journal of Agricultural Economics 70 (2021): 17-35.

RÜBCKE VON Veltheim, F., Claussen, F. und H. Heise (2021): Autonomous Field Robots in Agriculture: A Qualitative Analysis of User Acceptance According to Different Agricultural Machinery Companies. In: Schriften der Gesellschaft für Wirtschafts- und Sozialwissenschaften des Landbaus e.V. 56 (2021): 49-61. 
RÜBCKE VON VELTHEIM, F., ThEuVsen, L. und H. HeISE (2021): German Farmers' Intention to Use Autonomous Field Robots: A PLS Analysis. Dieser Beitrag wurde im August 2021 bei der wissenschaftlichen Zeitschrift Precision Agriculture zur Veröffentlichung angenommen.

\section{Beiträge in praxisorientierten Zeitschriften}

RÜBCKE VON VELTHEIM, F. und E. PIEPENBROCK (2019): RoboCop und Bumblebee für den Acker? In: f3 - farm. food. future. Interview. URL: https://f3.de/future/robocopund-bumblebee-fur-den-acker-439.html (Abrufdatum: 27.02.2021).

\section{Vorträge}

RÜBCKe VON Veltheim, F., Schaper, C. und H. Heise (2018): Die gesellschaftliche Wahrnehmung von bäuerlicher und industrieller Landwirtschaft. Vortrag auf der 28. Jahrestagung der Österreichischen Gesellschaft für Agrarökonomie. Wien, Österreich, 26.-28.09.2018.

RÜBCKE VON VELTHEIM, F. und H. HEISE (2019): Autonome Kleinmaschinen in der Landwirtschaft: Status quo. Vortrag auf der 39. Jahrestagung der Gesellschaft für Informatik in der Landwirtschaft (GIL). Wien, Österreich, 18. und 19.02.2019.

RÜBCKE VON Veltheim, F., Claussen, F. und H. Heise (2020): Autonomous Field Robots in Agriculture: A Qualitative Analysis of User Acceptance According to Different Agricultural Machinery Companies. Vortrag auf der 60. Jahrestagung der Gesellschaft für Wirtschafts- und Sozialwissenschaften des Landbaus e.V. (GEWISOLA). Halle/Saale, Deutschland, 23.-25.09.2020. 


\section{Danksagung}

„Dankbarkeit ist das Gedächtnis des Herzens. “(Jean-Baptiste Massillon, 1663-1742)

Es ist geschafft! Die vorliegende Dissertation ist das Ergebnis eines längeren Weges, den ich ohne die vielen begleitenden Gedanken, freundlichen Hinweise, geduldigen Ohren und aufbauenden Worte einer ganzen Reihe von lieben oder während dieser Zeit lieb gewonnenen Mitmenschen so sicher nicht hätte erfolgreich beenden können. Daher ist es mir ein großes Anliegen, mich bei all jenen aufrichtig zu bedanken.

Allen voran möchte ich meinem Doktorvater, Prof. Dr. Ludwig Theuvsen, danken. Danken für das mir entgegengebrachte Vertrauen, die vielen Freiheiten und die fachliche Begleitung bis zum Ende dieses besonderen Kapitels. Dies gilt umso mehr, da ihn sein Weg nach nur einem Jahr der gemeinsamen Zeit am Lehrstuhl in Göttingen, dem Ruf des Niedersächsischen Landwirtschaftsministeriums folgend, nach Hannover führte, die wissenschaftliche Begleitung aber aufrechterhielt. In gemeinsamen Gesprächen bewies er stets viel Fingerspitzengefühl, verstand es, Ideen in mir zu wecken und in Form zu gießen. Darüber hinaus und der Vielzahl an privaten und beruflichen Verpflichtungen zum Trotz bewies er immer wieder eine große und uns Doktoranden im positivsten Sinne ansteckende Lebensfreude sei es beim Karneval oder der alljährlichen Grünkohlwanderung unseres Lehrstuhls, an die ich noch lange und gerne zurückdenken werde.

Selbstverständlich möchte ich mich auch bei Prof. Dr. Achim Spiller für die Übernahme des Zweitgutachtens sowie bei Prof. Dr. Beneke, für die Komplettierung meines Prüfungsausschusses bedanken. Ein weiterer Dank gilt Prof. Dr. Plieninger für seine Beteiligung am Betreuungsausschuss der Dissertation.

In ganz besonderer Weise danke ich Frau Dr. Heinke Heise. Durch ihren unermüdlichen Einsatz, ihre hoch motivierten Sprachnachrichten, fachlich und menschlich wertvollen Hinweise und im festen Glauben an das Gelingen dieses Projektes hat sie außerordentlich zu dessen erfolgreichem Abschluss beigetragen. Es hat mich immer wieder in Erstaunen versetzt, mit welcher scheinbaren Leichtigkeit sie neben der eigenen jungen Familie meine Kommilitonen und mich so maßgeblich unterstützen konnte. Ihr empathisches, freundliches Wesen war uns Doktoranden im „blauen Turm“ stets ein willkommener Gast und brachte Klarheit in so manch gedankliches Wirrwarr. Daneben möchte ich Dr. Josef Langenberg gleich in zweierlei Hinsicht danken: zum einen für die wissenschaftliche Begleitung meiner Masterarbeit, zum anderen dafür, mir den entscheidenden Anstoß gegeben zu haben, den Weg eines Doktoranden zu beschreiten. Aber auch den anderen Mitgliedern des Teams „Betriebswirtschaftslehre im Agribusiness“ danke ich von Herzen für die vielen fröhlichen, unvergessenen Stunden in Göttingen. 
Nun bitte ich allen zuvor namentlich Genannten, es mir nachzusehen, dass der größte Dank meiner Familie gebührt. Worte werden dem Gefühl tiefer Dankbarkeit kaum gerecht, das ich für sie auch in Hinblick auf das eigene, bis hierin geschaffte Werden empfinde. So war meinen drei Geschwistern und mir nicht nur das Glück einer unbeschwerten Kindheit auf dem elterlichen Landwirtschaftsbetrieb in Vorpommern vergönnt, sondern es wurde uns auch das Privileg bedingungsloser Geborgenheit in den Herzen unserer Eltern zuteil. Sie haben mich immer in meinen Ideen unterstützt, sich viele meiner zum Teil verworrenen Gedanken geduldig angehört und jede Lebensphase freundlich begleitet. Ohne die vielen Freiheiten und ungezählten, wohlwollenden Worte und Taten hätte ich die vorliegende Arbeit in dieser Form sicher nicht zusammengetragen. Daher erscheint es mir das Mindeste, diese Arbeit jedem einzelnen Mitglied meiner geliebten Familie zu widmen. 


\section{Eidesstattliche Erklärungen}

Hiermit erkläre ich eidesstattlich, dass:

diese Arbeit weder in gleicher noch in ähnlicher Form bereits anderen Prüfungsbehörden vorgelegen hat.

ich mich an keiner anderen Hochschule um einen Doktorgrad beworben habe.

Göttingen, den 01. September 2021

(Unterschrift)

Hiermit erkläre ich eidesstattlich, dass diese Dissertation selbstständig und ohne unerlaubte Hilfe angefertigt wurde.

Göttingen, den 01. September 2021

(Unterschrift) 